\title{
Higher spin currents in Wolf space. Part I
}

\author{
Changhyun Ahn \\ Department of Physics, Kyungpook National University, \\ Taegu 702-701, Korea \\ E-mail: ahn@knu.ac.kr
}

AbStRaCt: For the $\mathcal{N}=4$ superconformal coset theory described by $\frac{\mathrm{SU}(N+2)}{\mathrm{SU}(N)}$ (that contains a Wolf space) with $N=3$, the $\mathcal{N}=2$ WZW affine current algebra with constraints is obtained. The 16 generators of the large $\mathcal{N}=4$ linear superconformal algebra are described by those WZW affine currents explicitly. By factoring out four spin- $\frac{1}{2}$ currents and the spin- 1 current from these 16 generators, the remaining 11 generators (spin- 2 current, four spin- $\frac{3}{2}$ currents, and six spin- 1 currents) corresponding to the large $\mathcal{N}=4$ nonlinear superconformal algebra are obtained.

Based on the recent work by Gaberdiel and Gopakumar on the large $\mathcal{N}=4$ holography, the extra 16 currents, with spin contents $\left(1, \frac{3}{2}, \frac{3}{2}, 2\right),\left(\frac{3}{2}, 2,2, \frac{5}{2}\right),\left(\frac{3}{2}, 2,2, \frac{5}{2}\right)$, and $\left(2, \frac{5}{2}, \frac{5}{2}, 3\right)$ described in terms of $\mathcal{N}=2$ multiplets, are obtained and realized by the WZW affine currents. As a first step towards $\mathcal{N}=4 \mathcal{W}$ algebra (which is NOT known so far), the operator product expansions (OPEs) between the above 11 currents and these extra 16 higher spin currents are found explicitly. It turns out that the composite fields with definite $\mathrm{U}(1)$ charges, made of above $(11+16)$ currents (which commute with the Wolf space subgroup $\mathrm{SU}(N=3) \times \mathrm{SU}(2) \times \mathrm{U}(1)$ currents $)$, occur in the right hand sides of these OPEs.

Keywords: AdS-CFT Correspondence, Conformal and W Symmetry

ARXIV EPRINT: 1311.6205 


\section{Contents}

1 Introduction 1

2 The large $\mathcal{N}=4$ (linear) superconformal algebra in the coset minimal model

$2.1 \mathcal{N}=2 \mathrm{WZW}$ affine current algebra

2.2 Large $\mathcal{N}=4$ linear superconformal algebra realization

2.2.1 Construction of two spin- $\frac{3}{2}$ currents

2.2.2 Construction of three spin-1 currents

2.2.3 Construction of four spin- $\frac{1}{2}$ currents and other two spin- $\frac{3}{2}$ currents 9

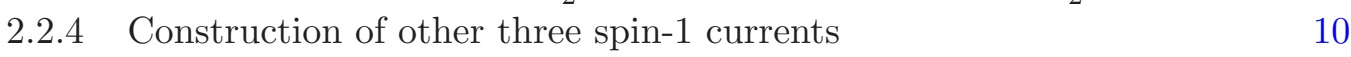

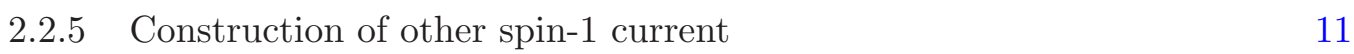

$\begin{array}{ll}2.2 .6 & \text { Construction of spin-2 current } \\ \end{array}$

3 The large $\mathcal{N}=4$ nonlinear superconformal algebra in the Wolf space coset minimal model

$\begin{array}{ll}3.1 \text { Construction of spin-2 stress tensor } & 14\end{array}$

$\begin{array}{lll}3.2 & \text { Construction of six spin-1 currents } & 14\end{array}$

$\begin{array}{ll}3.3 \text { Construction of four spin- } \frac{3}{2} \text { currents } & 16\end{array}$

$\begin{array}{lll}3.4 \mathrm{U}(1) \text { charge } & 18\end{array}$

4 Higher spin currents in the Wolf space coset minimal model 19

4.1 Construction of higher spin currents of spins $\left(1, \frac{3}{2}, \frac{3}{2}, 2\right) \quad 20$

4.2 Construction of higher spin currents of spins $\left(\frac{3}{2}, 2,2, \frac{5}{2}\right)$

4.3 Construction of higher spin currents of spins $\left(\frac{3}{2}, 2,2, \frac{5}{2}\right) \quad 26$

4.4 Construction of higher spin currents of spins $\left(2, \frac{5}{2}, \frac{5}{2}, 3\right) \quad 29$

5 Extension of large $\mathcal{N}=4$ nonlinear superconformal algebra in the coset minimal model $\quad 31$

$\begin{array}{lll}5.1 & \mathrm{U}(1) \text { charges of higher spin currents } & 32\end{array}$

5.2 Structure of OPEs 32

6 Conclusions and outlook $\quad 33$

A The OPEs of the large $\mathcal{N}=4$ nonlinear superconformal algebra from WZW affine currents

B The OPEs between the large $\mathcal{N}=4$ nonlinear algebra currents and the higher spin currents-I

B.1 The OPEs between six spin-1 currents and the higher spin current of spins $\left(1, \frac{3}{2}, \frac{3}{2}, 2\right)$ 
B.2 The OPEs between six spin 1 currents and two higher spin currents of spins $\left(\frac{3}{2}, 2,2, \frac{5}{2}\right)$

B.3 The OPEs between six spin 1 currents and the higher spin current of spins $\left(2, \frac{5}{2}, \frac{5}{2}, 3\right)$

B.4 The OPEs between six spin 1 currents and the higher spin currents in different basis

C The OPEs between the large $\mathcal{N}=4$ nonlinear algebra currents and the higher spin currents-II

C.1 The OPEs between four spin $\frac{3}{2}$ currents and the higher spin current of spins $\left(1, \frac{3}{2}, \frac{3}{2}, 2\right)$

C.2 The OPEs between four spin $\frac{3}{2}$ currents and two higher spin currents of spins $\left(\frac{3}{2}, 2,2, \frac{5}{2}\right)$

C.3 The OPEs between four spin $\frac{3}{2}$ currents and the higher spin current of spins $\left(2, \frac{5}{2}, \frac{5}{2}, 3\right)$

C.4 The OPEs between four spin $\frac{3}{2}$ currents and the higher spin currents in different basis

\section{Introduction}

Gaberdiel and Gopakumar [1] have proposed that the large $\mathcal{N}=4$ higher spin theory on $A d S_{3}$ based on the higher spin algebra is dual to the 't Hooft limit of the two dimensional large $\mathcal{N}=4$ superconformal coset theory. The $\mathcal{N}=4$ higher spin algebra contains 8 fields of spin $s=\frac{3}{2}, 2, \frac{5}{2}, \cdots$ and 7 fields of spin $s=1$ and the exceptional superalgebra $D\left(2,1 \mid \frac{\mu}{1-\mu}\right)$ is the largest finite dimensional subalgebra of this $\mathcal{N}=4$ higher spin algebra. The $\mathcal{N}=4$ coset theory is described by the coset $\frac{\mathrm{SU}(N+2)}{\mathrm{SU}(N)}$ with the level which is equal to the sum of each level of two $\mathrm{SU}(2)$ affine algebras. The 't Hooft coupling constant $\lambda$ is identified with the free parameter of the large $\mathcal{N}=4$ superconformal algebra (this additional parameter is due to the fact that there exist two SU(2) affine algebras rather than one) and furthermore this 't Hooft parameter $\lambda$ is equal to the above $\mu$ parameter which is related to the mass of scalar field in the $A d S_{3}$ bulk theory, according to this proposal [1]. See also previous works on the minimal model holography [2-5].

More specifically, the large $\mathcal{N}=4$ linear superconformal algebra is generated by spin- 2 stress tensor, four spin- $\frac{3}{2}$ supersymmetry generators, seven spin-1 currents (six of them are the generators of two $\mathrm{SU}(2)$ affine algebras and one of them is $\mathrm{U}(1)$ current) and four spin- $\frac{1}{2}$ currents [6]. Although the wedge algebra of the large $\mathcal{N}=4$ linear superconformal algebra is also $D\left(2,1 \mid \frac{\mu}{1-\mu}\right)$ together with a central generator, the large $\mathcal{N}=4$ linear superconformal algebra is not a subalgebra of the extended algebra of higher spin currents because four fermions of spin- $\frac{1}{2}$ do not occur in the above higher spin algebra. By factoring out the spin- 1 current and four spin- $\frac{1}{2}$ currents from the above large $\mathcal{N}=4$ linear superconformal algebra, the remaining $11(=16-5)$ generators consist of the nonlinear version of the large 
$\mathcal{N}=4$ linear superconformal algebra [7]. ${ }^{1}$ The levels of two SU(2) affine algebras are reduced by one and the central charge appearing in the Virasoro algebra is reduced by three. Furthermore the central term in the operator product expansions (OPE) between the spin- $\frac{3}{2}$ currents is also changed and the quadratic terms in the first-order poles of the OPEs occur.

In [15], the $\mathcal{N}=4$ extension of Kazama-Suzuki model $[16,17]$ is described and the $\mathcal{N}=4$ coset model has the form Wolf $\times \mathrm{SU}(2) \times \mathrm{U}(1)$ where Wolf is a Wolf space (or quaternion-Kahler symmetric space) $[18-20]{ }^{2}$ The fourteen currents of the large $\mathcal{N}=4$ linear superconformal algebra are expressed in terms of affine Kac-Moody currents (the remaining two currents can be obtained from the $\mathcal{N}=1$ Sugawara construction) and the relative tensorial structures appearing in these expressions satisfy the particular identities. They also found the nonlinear algebra by factoring out the spin- 1 and spin- $\frac{1}{2}$ currents that live in the $\mathrm{U}(1)$ and the coset is given by Wolf $\times \mathrm{SU}(2)$. The remaining three spin- $\frac{1}{2}$ currents living in $\mathrm{SU}(2)$ can be decoupled further.

For the simplest case where the $\mathcal{N}=4$ coset is given by $\mathrm{SU}(2) \times \mathrm{U}(1)[22,23]$ corresponding to the first entry of the table 1 in [15], using the above identities between the tensorial structures, the 16 generators of the large $\mathcal{N}=4$ linear superconformal algebra are written in terms of the affine Kac-Moody currents with constraints in $\mathcal{N}=2$ superspace. $^{3}$ Furthermore, following the work of [15], by imposing on the above large $\mathcal{N}=4$ nonlinear superconformal algebra in the $\mathcal{N}=4$ coset theory, the coset turns out to be a Wolf space itself in [24].

In this paper, we would like to construct the 16 generators of the large $\mathcal{N}=4$ linear superconformal algebra in the $\operatorname{coset}^{4}$

$$
\text { Wolf } \times \mathrm{SU}(2) \times \mathrm{U}(1)=\frac{\mathrm{SU}(N+2)}{\mathrm{SU}(N)}
$$

(that is the third entry of the table 1 in [15]) theory with $N=3$. By factoring out the spin-1 current and four spin- $\frac{1}{2}$ currents, the 11 generators of the large $\mathcal{N}=4$ nonlinear algebra in the Wolf space coset are obtained. ${ }^{5}$ One of the findings in [1] is that the lowest

\footnotetext{
${ }^{1}$ Sometimes this algebra is called by $\mathcal{N}=4$ quasi superconformal algebra because this is generated quadratically (nonlinearly). On the other hand, the small (or regular) $\mathcal{N}=4$ superconformal algebra $[8,9]$ can be obtained by taking one of the level as zero and the other level as an infinity in the large $\mathcal{N}=4$ linear superconformal algebra (therefore there exists only a single $\mathrm{SU}(2)$ affine algebra rather than two). Also in [10], the large $\mathcal{N}=4$ linear superconformal algebra was studied. See also [11-14].

${ }^{2}$ The Wolf space appeared in $\mathcal{N}=2$ supergravity in four dimensions [21].

${ }^{3}$ Although we use the $\mathcal{N}=2$ description in this paper, due to the constraints for the $\mathcal{N}=2$ WZW affine currents, effectively our description is the same as the $\mathcal{N}=1$ approach in [15].

${ }^{4}$ Of course, the bosonic coset can be obtained by introducing the extra $\mathrm{SO}(4 N+4)$ (generated by $(4 N+4)$ free fermions) at level 1 in the numerator of the coset as in [1, 25]. The number $(4 N+4)$ is the dimension of the coset $\frac{\mathrm{SU}(N+2)}{\mathrm{SU}(N)}$. That is, $(N+2)^{2}-1-\left(N^{2}-1\right)=4 N+4$.

${ }^{5}$ Let us emphasize that one can combine the spin-1 current and one spin- $\frac{1}{2}$ current and express them as a single $\mathcal{N}=1$ super current which corresponds to the $\mathrm{U}(1)$ factor in the left hand side of (1.1). However, the remaining three spin- $\frac{1}{2}$ currents are part of the $\mathcal{N}=1$ superconformal affine $\mathrm{SU}(2)$ algebra. In other words, the superpartners of these three spin- $\frac{1}{2}$ currents, i.e. three spin- 1 currents do not play an important role in the denominator subgroup. When one divides $\mathrm{SU}(2) \times \mathrm{U}(1)$ factor both sides of (1.1), then one obtains Wolf $=\frac{\mathrm{SU}(N+2)}{\mathrm{SU}(N) \times \mathrm{SU}(2) \times \mathrm{U}(1)}$ where the $\mathrm{SU}(2)$ factor in the denominator refers to only the above three spin- $\frac{1}{2}$ currents. Their superpartners, three spin- 1 currents appear in the group $\mathrm{SU}(N+2)$.
} 
nontrivial multiplet of the higher spin algebra has one spin-1 current, four spin- $\frac{3}{2}$ currents, six spin-2 currents, four spin- $\frac{5}{2}$ currents and one spin-3 current and let us denote them by spin contents as follows:

$$
\left(1, \frac{3}{2}, \frac{3}{2}, 2\right), \quad\left(\frac{3}{2}, 2,2, \frac{5}{2}\right), \quad\left(\frac{3}{2}, 2,2, \frac{5}{2}\right), \quad\left(2, \frac{5}{2}, \frac{5}{2}, 3\right) .
$$

We construct these 16 currents (1.2) in terms of $\mathcal{N}=2$ affine Kac-Moody currents in the above Wolf space coset theory explicitly. Furthermore, we calculate the various OPEs between the 11 generators of the large $\mathcal{N}=4$ nonlinear superconformal algebra and the 16 higher spin currents. ${ }^{6}$

By construction, the 16 higher spin currents (anti)commute with the eight spin- $\frac{1}{2}$ currents (and its superpartner eight spin-1 currents) living in the effective $\mathcal{N}=1$ subgroup $\mathrm{SU}(N=3)$ of the Wolf space coset, the three spin- $\frac{1}{2}$ currents living in the bosonic subgroup $\mathrm{SU}(2)$ of the Wolf space coset and the remaining spin- $\frac{1}{2}$ current and its superpartner spin1 current living in the effective $\mathcal{N}=1$ subgroup $\mathrm{U}(1)$ of the Wolf space coset. The 16 currents are primary under the stress energy tensor of $\mathcal{N}=4$ nonlinear superconformal algebra.

In the OPEs between the four spin- $\frac{3}{2}$ currents and 16 higher spin currents, the right hand sides of these OPEs have special features. If one describes (1.2) as four rows for $4 \times 4$ matrix, then one writes the OPEs between the above four spin- $\frac{3}{2}$ currents and this $4 \times 4$ matrix which has 16 components. Let us concentrate on the linear terms on the higher spin current appearing in the right hand side of OPEs (There are also linear or nonlinear terms containing 11 currents of large $\mathcal{N}=4$ nonlinear superconformal algebra in the full expressions). It turns out that there are no higher spin currents, at the linear level, in the second row, third column, second column and third row in the above each $4 \times 4$ matrix, respectively. These vanishings of the rows and columns are quite related to the locations of four spin- $\frac{3}{2}$ currents in the $\mathcal{N}=2$ superspace multiplets. One can describe the 16 currents of large $\mathcal{N}=4$ linear superconformal algebra as $4 \times 4$ matrix also as done for 16 higher spin currents: $\left(1, \frac{3}{2}, \frac{3}{2}, 2\right),\left(\frac{1}{2}, 1,1, \frac{3}{2}\right),\left(\frac{1}{2}, 1,1, \frac{3}{2}\right)$ and $\left(0, \frac{1}{2}, \frac{1}{2}, 1\right)$. The first, second, third and fourth spin- $\frac{3}{2}$ currents are $(1,2),(1,3),(2,4)$ and $(3,4)$ elements of $4 \times 4$ matrix and play the role of vanishing second column, third column, second row and third row above respectively. ${ }^{7}$

In section 2 , the 16 currents of $\mathcal{N}=4$ linear superconformal algebra are obtained in the coset model (1.1).

In section 3 , the 11 currents of $\mathcal{N}=4$ nonlinear superconformal algebra are determined in the Wolf space coset (1.1).

\footnotetext{
${ }^{6}$ The $\mathcal{N}=4 W_{3}$ algebra in different context was considered in [26, 27]. One example of $W$ algebra with Wolf space was found in [28]. Recently, the higher spin theory with extended supersymmetries where the two dimensional coset theory contains Wolf space was studied in [29].

${ }^{7}$ That is, when the particular spin- $\frac{3}{2}$ current acts on the 16 higher spin currents described by $4 \times 4$ matrix, one does not see any higher spin currents in the given row or column containing that spin- $\frac{3}{2}$ current in $4 \times 4$ matrix. For example, for the first case where the spin- $\frac{3}{2}$ current is an $(2,4)$ element of $4 \times 4$ matrix, the second multiplet of (1.2) appears in the first- and fourth-rows in the right hand side of OPEs and both first- and fourth-multiplets of (1.2) appear in the third row in the right hand side of OPEs:(6.1). One sees the vanishing of second row in the right hand side of OPEs. See the section 6 for more detailed descriptions.
} 
In section 4 , the extra 16 currents in (1.2) are obtained in the Wolf space coset (1.1). ${ }^{8}$

In section 5, the OPEs between the 11 currents in section 3 and 16 currents in section 4 are obtained.

In section 6, we summarize what has been done in this paper.

In appendices $\mathrm{A}-\mathrm{C}$, some details which are necessary in sections $2-5$ are presented. They contain the complete higher spin algebra.

The mathematica package by Thielemans [30] is used all the times.

\section{The large $\mathcal{N}=4$ (linear) superconformal algebra in the coset minimal model}

Before we construct the large $\mathcal{N}=4$ nonlinear superconformal algebra using the $\mathcal{N}=2$ WZW affine currents, we would like to describe its linear version first. After that, in next section, the large $\mathcal{N}=4$ nonlinear superconformal algebra will be constructed explicitly.

\section{1 $\mathcal{N}=2$ WZW affine current algebra}

Let us consider the particular $\mathcal{N}=4$ superconformal coset theory described in $[1,15]$ which can be described as $^{9}$

$$
\text { Wolf } \times \mathrm{SU}(2) \times \mathrm{U}(1)=\frac{\mathrm{SU}(N+2)}{\mathrm{SU}(N)}, \quad \text { where } \quad \text { Wolf }=\frac{\mathrm{SU}(N+2)}{\mathrm{SU}(N) \times \mathrm{SU}(2) \times \mathrm{U}(1)} .
$$

The central charge of this coset model [16] is given by

$$
\begin{aligned}
c_{\mathrm{SU}(N+2)}-c_{\mathrm{SU}(N)} & =\frac{3}{2}\left[(N+2)^{2}-1\right]\left[1-\frac{2(N+2)}{3(k+N+2)}\right]-\frac{3}{2}\left[N^{2}-1\right]\left[1-\frac{2 N}{3(k+N+2)}\right] \\
& =\frac{6(k+1)(N+1)}{(k+N+2)} .
\end{aligned}
$$

The level of the currents in the numerator and denominator of the coset model (2.1) is $(k+N+2)$ respectively. Note that the general expression of the central charge of the large $\mathcal{N}=4$ linear superconformal algebra $c=\frac{6 k^{+} k^{-}}{\left(k^{+}+k^{-}\right)}$where $k^{+}=(k+1)$ and $k^{-}=(N+1)$ are the levels of two $\mathrm{SU}(2)$ affine algebras. We will see that the central charge (2.2) occurs in the OPE between the spin- 2 currents (and the OPE between the spin- $\frac{3}{2}$ currents) in the large $\mathcal{N}=4$ linear superconformal algebra.

Furthermore, the central charge of the Wolf space (for the time being we assume the contributions from the superpartners of three spin- $\frac{1}{2}$ fermions described in the footnote 5 )

\footnotetext{
${ }^{8}$ When we say "the higher spin currents" in this paper, they are given by these 16 currents (1.2). Some of the spins are less than 2. In other words, they consist of all the extra currents besides the large $\mathcal{N}=4$ nonlinear algebra currents.

${ }^{9}$ Usually the U(1) factor in (2.1) in the denominator of Wolf space is missing for other types of Wolf space. The details for using this U(1) factor to other types are given in [31]. Furthermore, one can see the related works on other types (orthogonal or noncompact) of Wolf space in [32, 33].
} 
can be obtained as follows:

$$
\begin{aligned}
c_{\mathrm{Wolf}} & =c_{\mathrm{SU}(N+2)}-c_{\mathrm{SU}(N)}-c_{\mathrm{SU}(2)}-c_{\mathrm{U}(1)} \\
& =\frac{6(k+1)(N+1)}{(k+N+2)}-\frac{3}{2} \times 3\left[1-\frac{4}{3(k+N+2)}\right]-\frac{3}{2} \\
& =\frac{6 k N}{(2+k+N)},
\end{aligned}
$$

where the above level $(k+N+2)$ appearing in the coset (2.1) is taken in the $\mathrm{SU}(2)$ factor in the denominator in (2.1). In the second line of (2.3), the result of (2.2) is used. Compared to the central charge in the large $\mathcal{N}=4$ linear superconformal algebra, this central charge will appear in the OPE between the two spin- $\frac{3}{2}$ currents in the large $\mathcal{N}=4$ nonlinear superconformal algebra in next section. For the other central term appearing in the stress tensor, the contribution from the central charge $c_{\mathrm{SU}(2)}$ in $(2.3)$ will be $-\frac{3}{2} \cdot{ }^{10}$

We would like to construct the large $\mathcal{N}=4$ linear superconformal algebra for the coset theory (2.1). For the $\mathcal{N}=2$ currents where the $\mathcal{N}=2$ superspace coordinate $Z=(z, \theta, \bar{\theta})$ is given by the bosonic coordinate $z$ and two Grassman coordinates $\theta$ and $\bar{\theta}$, the component currents are given by ${ }^{11}$

$$
\begin{aligned}
K^{m}(Z) & =K^{m}(z)+\left.\theta D K^{m}\right|_{\theta=\bar{\theta}=0}(z)+\left.\bar{\theta} \bar{D} K^{m}\right|_{\theta=\bar{\theta}=0}(z)+\left.\theta \bar{\theta}(-1) \frac{1}{2}[D, \bar{D}] K^{m}\right|_{\theta=\bar{\theta}=0}(z), \\
K^{\bar{m}}(Z) & =K^{\bar{m}}(z)+\left.\theta D K^{\bar{m}}\right|_{\theta=\bar{\theta}=0}(z)+\left.\bar{\theta} \bar{D} K^{\bar{m}}\right|_{\theta=\bar{\theta}=0}(z)+\left.\theta \bar{\theta}(-1) \frac{1}{2}[D, \bar{D}] K^{\bar{m}}\right|_{\theta=\bar{\theta}=0}(z), \\
J^{a}(Z) & =J^{a}(z)+\left.\theta D J^{a}\right|_{\theta=\bar{\theta}=0}(z)+\left.\bar{\theta} \bar{D} J^{a}\right|_{\theta=\bar{\theta}=0}(z)+\left.\theta \bar{\theta}(-1) \frac{1}{2}[D, \bar{D}] J^{a}\right|_{\theta=\bar{\theta}=0}(z), \\
J^{\bar{a}}(Z) & =J^{\bar{a}}(z)+\left.\theta D J^{\bar{a}}\right|_{\theta=\bar{\theta}=0}(z)+\left.\bar{\theta} \bar{D} J^{\bar{a}}\right|_{\theta=\bar{\theta}=0}(z)+\left.\theta \bar{\theta}(-1) \frac{1}{2}[D, \bar{D}] J^{\bar{a}}\right|_{\theta=\bar{\theta}=0}(z), \quad(2.6)
\end{aligned}
$$

where two complex spinor covariant derivatives $D$ and $\bar{D}$ satisfy the algebra $D \bar{D}+\bar{D} D=$ $-\partial_{z}$. The $\mathrm{SU}(N+2)$ indices are decomposed into the $\mathrm{SU}(N+1)$ indices and others: the former is denoted by $m, \bar{m}$ and the latter is denoted by $a, \bar{a}$. We will come to this issue of the indices soon.

${ }^{10}$ As described in [1], the Wolf space can be generalized to the following coset model

$$
\frac{\mathrm{SU}(N+M)}{\mathrm{SU}(N) \times \mathrm{SU}(M) \times \mathrm{U}(1)},
$$

which appears in the standard $\mathcal{N}=2$ Kazama-Suzuki model $[16,17]$. Then the central charge of this coset (2.4) can be obtained from

$$
\begin{aligned}
c_{K S}= & c_{\mathrm{SU}(N+M)}-c_{\mathrm{SU}(N)}-c_{\mathrm{SU}(M)}-c_{\mathrm{U}(1)} \\
= & \frac{3}{2}\left[(N+M)^{2}-1\right]\left[1-\frac{2(N+M)}{3(k+N+M)}\right]-\frac{3}{2}\left[N^{2}-1\right]\left[1-\frac{2 N}{3(k+N+M)}\right] \\
& -\frac{3}{2}\left[M^{2}-1\right]\left[1-\frac{2 M}{3(k+N+M)}\right]-\frac{3}{2}=\frac{3 k M N}{(k+M+N)},
\end{aligned}
$$

where the level in each factor of the coset is given by $(k+N+M)$. Of course, this will become the previous central charge (2.3) for $M=2$.

${ }^{11}$ One finds the explicit component expressions in (19) of [22] or (24) of [34]. 
The nonlinear constraints, by taking $\theta, \bar{\theta}$ independent terms from $(2.8)$ of $[34,35]$, are given by ${ }^{12}$

$$
\begin{gathered}
\left.D K^{m}\right|_{\theta=\bar{\theta}=0}(z)=-\left.\frac{1}{2(k+N+2)} f_{\bar{m} n}^{\bar{p}}\left(K^{n} K^{p}\right)\right|_{\theta=\bar{\theta}=0}(z), \\
\left.D J^{a}\right|_{\theta=\bar{\theta}=0}(z)=-\left.\frac{1}{(k+N+2)} f_{\bar{a} b} \bar{m}^{\bar{m}}\left(J^{b} K^{m}\right)\right|_{\theta=\bar{\theta}=0}(z), \\
\left.\bar{D} K^{\bar{m}}\right|_{\theta=\bar{\theta}=0}(z)=-\left.\frac{1}{2(k+N+2)} f_{m \bar{n}}\left(K^{\bar{n}} K^{\bar{p}}\right)\right|_{\theta=\bar{\theta}=0}(z), \\
\left.\bar{D} J^{\bar{a}}\right|_{\theta=\bar{\theta}=0}(z)=-\left.\frac{1}{(k+N+2)} f_{a \bar{b}}^{m}\left(J^{\bar{b}} K^{\bar{m}}\right)\right|_{\theta=\bar{\theta}=0}(z) .
\end{gathered}
$$

Then the $\theta$ - and $\theta \bar{\theta}$-components of $K^{m}(Z)$ and $J^{a}(Z)$ in (2.6) are not independent and they can be written in terms of the $\theta, \bar{\theta}$-independent term and $\bar{\theta}$-components according to (2.8) and (2.7). Similarly, the $\bar{\theta}$-and $\theta \bar{\theta}$-components of $K^{\bar{m}}(Z)$ and $J^{\bar{a}}(Z)$ in (2.6) can be written in terms of the $\theta, \bar{\theta}$-independent term and $\theta$-components. ${ }^{13}$ The on-shell current algebra in $\mathcal{N}=2$ superspace for the supersymmetric WZW model on a group $\mathrm{SU}(N+2)$ of even dimension can be written in terms of components of spin $s=\frac{1}{2}, 1$ [35].

For $N=3$, the 24 adjoint indices of SU(5) are divided into 12 unbarred indices and 12 barred indices. Then the 8 adjoint indices of the subgroup $\mathrm{SU}(3)$ are given by $4,5,6$ and some combination between the index 7 and the index 8 (and their barred indices). ${ }^{14}$ Then the remaining indices, 1, 2, 3, 9, 10,11, 12 and some combination between the index 7 and the index 8 (and their barred indices) live in the coset $\frac{\mathrm{SU}(5)}{\mathrm{SU}(3)}$. We follow the convention of [35]. However, some currents $\left(K^{m}\right.$ or $\left.K^{\bar{m}}\right)$ living in the subgroup SU(4) of [35] live in the coset $\frac{\mathrm{SU}(5)}{\mathrm{SU}(3)}$. In other words, one classifies the 24 basic $\mathcal{N}=2$ WZW affine currents as

$$
\begin{aligned}
& { }^{12} \text { Then it is easy to obtain the following relations, which can be obtained from (2.9) of [35] by putting } \\
& \theta=\bar{\theta}=0, \\
& \left.\quad[D, \bar{D}] K^{m}\right|_{\theta=\bar{\theta}=0}(z)=-\left.\partial K^{m}\right|_{\theta=\bar{\theta}=0}(z)+\left.\frac{1}{(k+N+2)} f_{\bar{m} n} \bar{p}\left(\bar{D} K^{n} K^{p}-K^{n} \bar{D} K^{p}\right)\right|_{\theta=\bar{\theta}=0}(z), \\
& {\left.[D, \bar{D}] K^{\bar{m}}\right|_{\theta=\bar{\theta}=0}(z)=\left.\partial K^{\bar{m}}\right|_{\theta=\bar{\theta}=0}(z)-\left.\frac{1}{(k+N+2)} f_{m \bar{n}}{ }^{p}\left(D K^{\bar{n}} K^{\bar{p}}-K^{\bar{n}} D K^{\bar{p}}\right)\right|_{\theta=\bar{\theta}=0}(z),} \\
& {\left.[D, \bar{D}] J^{a}\right|_{\theta=\bar{\theta}=0}(z)=-\left.\partial J^{a}\right|_{\theta=\bar{\theta}=0}(z)+\left.\frac{2}{(k+N+2)} f_{\bar{a} b} \bar{m}^{\bar{m}}\left(\bar{D} J^{b} K^{m}-J^{b} \bar{D} K^{m}\right)\right|_{\theta=\bar{\theta}=0}(z),} \\
& {\left.[D, \bar{D}] J^{\bar{a}}\right|_{\theta=\bar{\theta}=0}(z)=\left.\partial J^{\bar{a}}\right|_{\theta=\bar{\theta}=0}(z)-\left.\frac{2}{(k+N+2)} f_{a \bar{b}}{ }^{m}\left(D J^{\bar{b}} K^{\bar{m}}-J^{\bar{b}} D K^{\bar{m}}\right)\right|_{\theta=\bar{\theta}=0}(z) .}
\end{aligned}
$$

\footnotetext{
${ }^{13}$ As one computes the OPEs in the component approach in the whole paper, for simplicity, one ignores the notation $\left.\right|_{\theta=\bar{\theta}=0}$ acting on the $\mathcal{N}=2$ superfields from now on [36]. That is, $\left.D K^{\bar{m}}\right|_{\theta=\bar{\theta}=0}(z) \equiv$ $D K^{\bar{m}}(z),\left.\bar{D} K^{m}\right|_{\theta=\bar{\theta}=0}(z) \equiv \bar{D} K^{m}(z),\left.D J^{\bar{a}}\right|_{\theta=\bar{\theta}=0}(z) \equiv D J^{\bar{a}}(z)$, and $\left.\bar{D} J^{a}\right|_{\theta=\bar{\theta}=0}(z) \equiv \bar{D} J^{a}(z)$.

${ }^{14}$ The convention of [1] for the subgroup SU(3), around (3.1) of [1], is different from ours because the first $3 \times 3$ block of $5 \times 5$ corresponds to the their subgroup $\mathrm{SU}(3)$ (the unbarred indices 1,2, 4 and some combination between the index 7 and index 8 and their barred ones will describe their SU(3)) while the middle $3 \times 3$ block of $5 \times 5$ corresponds to our subgroup SU(3). Moreover, the adjoint representation 24 of $\mathrm{SU}(5)$ breaks into $\mathbf{2 4} \rightarrow(\mathbf{8}, \mathbf{1}) \oplus(\mathbf{1}, \mathbf{3}) \oplus(\mathbf{1}, \mathbf{1}) \oplus(\mathbf{3}, \mathbf{2}) \oplus(\overline{\mathbf{3}}, \mathbf{2})$ under the $\mathrm{SU}(3) \times \mathrm{SU}(2)$. The first three representations are given by the currents with the indices $4,5,6,7,8$ and 9 (and conjugated ones). The last two representations are given by the currents with indices 1,2,3 and 10,11,12 (and conjugated ones). Note that the subgroup SU(4) having the indices $1, \cdots, 8$ (and conjugated ones) cannot be broken into $\mathrm{SU}(3) \times \mathrm{SU}(2)$.
} 
follows:

$$
\begin{array}{rlrl}
K^{m} & =\left(K^{1}, K^{2}, K^{3} ; K^{4}, K^{5}, K^{6}, K^{7}, K^{8}\right): & \text { coset and subgroup currents, } \\
K^{\bar{m}} & =\left(K^{\overline{1}}, K^{\overline{2}}, K^{\overline{3}} ; K^{\overline{4}}, K^{\overline{5}}, K^{\overline{6}}, K^{\overline{7}}, K^{\overline{8}}\right): & \text { coset and subgroup currents, } \\
J^{a} & =\left(J^{9}, J^{10}, J^{11}, J^{12}\right): & \text { coset currents, } & \\
J^{\bar{a}} & =\left(J^{\overline{9}}, J^{\overline{10}}, J^{11}, J^{\overline{12}}\right): \quad \quad \text { coset currents. }
\end{array}
$$

Of course, one can write down the currents having the indices 1,2,3 (and their barred indices) $J^{1}, J^{2}$ and $J^{3}$ (and their barred currents) respectively but one cannot split the currents having the indices 7 and 8 in terms of coset and subgroup currents at the moment.

For the Wolf $\times \mathrm{SU}(2) \times \mathrm{U}(1)=\mathrm{SU}(2) \times \mathrm{U}(1)$ WZW model with $k^{+}=k+1$ and $k^{-}=1$, as described in the introduction, the 16 generators of the large $\mathcal{N}=4$ linear superconformal algebra are expressed in terms of $\mathcal{N}=2$ super stress energy tensor of super spin- 1 , two super spin- $\frac{1}{2}$ currents and a super spin-0 current [22]. In other words, the total 16 currents are given by their spin contents

$$
\left(1, \frac{3}{2}, \frac{3}{2}, 2\right), \quad\left(\frac{1}{2}, 1,1, \frac{3}{2}\right), \quad\left(\frac{1}{2}, 1,1, \frac{3}{2}\right), \quad\left(0, \frac{1}{2}, \frac{1}{2}, 1\right) .
$$

Note that the spin- 1 current appears as a derivative of spin- 0 field which is the first component field of the last $\mathcal{N}=2$ super current of (2.10). See also relevant work [37] where the $\mathrm{SU}(2) \times \mathrm{U}(1)$ group appears in the subgroup of the coset model.

\subsection{Large $\mathcal{N}=4$ linear superconformal algebra realization}

We would like to construct the 16 generators in terms of the numerator $\mathrm{SU}(5)$ currents in the coset (2.1) explicitly.

\subsubsection{Construction of two spin- $\frac{3}{2}$ currents}

Let us consider the spin- $\frac{3}{2}$ current which is the last component field of the second $\mathcal{N}=2$ super current in (2.10). Among the coset indices of 1,2,3, 9, 10,11, 12 and some combination of the index 7 and the index 8 , it is natural to consider the index 9 corresponding to the above spin- $\frac{3}{2}$ current by taking the appropriate derivative or spinor covariant derivatives. One can write down the following quantity ${ }^{15}$

$$
G_{11}(z)=\sqrt{2} i\left(-\frac{1}{2}[D, \bar{D}] J^{9}-\frac{(k-3)}{2(5+k)} \partial J^{9}\right)(z) .
$$

\footnotetext{
${ }^{15}$ We denote the four spin- $\frac{3}{2}$ currents $G_{11}(z), G_{12}(z), G_{21}(z)$ and $G_{22}(z)$ here, along the line of [38], for the $G_{+K}(z), G_{+}(z), G_{-}(z)$ and $G_{-K}(z)$ in [39]. That is, the vector (or fundamental) representation of $\mathrm{SO}(4)$ is written in terms of two $\mathrm{SU}(2)$ 's fundamentals. The three spin-1 currents $A^{+i}(z)$ where $i=1,2,3$ in [39] correspond to $A_{1}(z), A_{2}(z)$ and $A_{3}(z)$ here. The three spin-1 currents $A^{-i}(z)$ of spin-1 over there correspond to $B_{1}(z), B_{2}(z)$ and $B_{3}(z)$ here. The four spin- $\frac{1}{2}$ currents $\Gamma_{+K}(z), \Gamma_{+}(z), \Gamma_{-}(z)$ and $\Gamma_{-K}(z)$ over there correspond to $F_{11}(z), F_{12}(z), F_{21}(z)$ and $F_{22}(z)$ with two $\mathrm{SU}(2)$ 's. We use the same notations for the spin- 2 and the spin- 1 currents as $T(z)$ and $\mathrm{U}(z)$ respectively.
} 
With the second term of (2.11), the $G_{11}(z)$ transforms as a primary field under the stress energy tensor $T_{\mathrm{SU}(5)}{ }^{16}$ The coefficient $\frac{(k-3)}{(5+k)}$ can be written as $\frac{\left(k^{+}-k^{-}\right)}{\left(k^{+}+k^{-}\right)}$with $k^{+}=k+1$ and $k^{-}=N+1=4^{17}$

Similarly, one can construct the following primary field of spin- $\frac{3}{2}$, corresponding to the last component field of the third $\mathcal{N}=2$ super current in (2.10), under the $T_{\mathrm{SU}(5)}$ as follows: ${ }^{18}$

$$
G_{22}(z)=\sqrt{2} i\left(-\frac{1}{2}[D, \bar{D}] J^{\overline{9}}+\frac{(k-3)}{2(5+k)} \partial J^{\overline{9}}\right)(z) .
$$

\subsubsection{Construction of three spin-1 currents}

What about the spin- 1 currents which are the superpartner of the above spin- $\frac{3}{2}$ currents $G_{11}$ and $G_{22}$ ? One expects that two linear combinations between $\bar{D} J^{9}$ and $D J^{\overline{9}}$ provide the two spin- 1 currents. Note that there are no singular terms in the OPEs $D J^{\overline{9}}(z) D J^{\overline{9}}(w)$ and $\bar{D} J^{9}(z) \bar{D} J^{9}(w)$. With the identifications

$$
\begin{aligned}
& A_{1}(z)=\frac{1}{2}\left(-\bar{D} J^{9}+D J^{\overline{9}}\right)(z), \\
& A_{2}(z)=\frac{i}{2}\left(\bar{D} J^{9}+D J^{\overline{9}}\right)(z)
\end{aligned}
$$

one calculates the following OPEs

$$
A_{i}(z) A_{i}(w)=\frac{1}{(z-w)^{2}}(-1) \frac{1}{2}(k+1)+\cdots, \quad i=1,2,
$$

where the second order pole can be written as $-\frac{1}{2} k^{+}$because of $k^{+}=(k+1)$.

Let us calculate the third spin- 1 current by calculating the OPE $A_{1}(z) A_{2}(w)$ with $(2.13)$. It turns out that

$$
A_{1}(z) A_{2}(w)=\frac{1}{(z-w)} A_{3}(w)+\cdots
$$

where the spin-1 current is given by

$$
A_{3}(w)=-\frac{i}{2(5+k)} \sum_{(a, \bar{a})=(9, \overline{9})}^{(12, \overline{1})} J^{a} J^{\bar{a}}(w)+\text { other quadratic and linear terms. }
$$

\footnotetext{
${ }^{16}$ Among $\mathcal{N}=2 \mathrm{WZW}$ affine currents, the primary currents under the spin-2 stress tensor $\left(T_{\mathrm{SU}(5)}-\right.$ $T_{\mathrm{SU}(3))}(z)$ are given by $J^{9}(w)$ and $J^{\overline{9}}(w)$. Here $T_{\mathrm{SU}(5)}$ can be obtained from the last component of $\mathcal{N}=2$ superfield $\frac{1}{2(N+2+k)}[D, \bar{D}]\left(J^{a} J^{\bar{a}}+K^{m} K^{\bar{m}}-\left(f_{\bar{m} \bar{a}} \bar{a}+f_{\bar{m} \bar{n}}\right) D K^{\bar{m}}-\left(f_{m \bar{a}}+f_{m \bar{n}}{ }^{\bar{n}}\right) \bar{D} K^{m}\right)(Z)$ [35] which is equal to $T_{\mathrm{SU}(5)}(z)=\frac{1}{(5+k)^{3}}\left(K^{1} K^{4} K^{\overline{1}} K^{\overline{4}}+\frac{i}{2} K^{1} K^{4} K^{\overline{2}} K^{\overline{7}}\right)+$ other quartic, cubic, quadratic and linear terms.Similarly, one has $T_{\mathrm{SU}(3)}=\frac{1}{(5+k)^{3}}\left(K^{4} K^{6} K^{\overline{4}} K^{\overline{6}}+\frac{i}{2} K^{4} K^{6} K^{\overline{5}} K^{\overline{7}}\right)+$ other quartic, cubic, quadratic and linear terms. The indices for the fields in $T_{\mathrm{SU}(3)}(z)$ are given by $4,5,6$ and 7 and 8 (and their conjugated ones).

${ }^{17} \mathrm{By}$ substituting $(2.7)$ into the equation $(2.11)$, one obtains $G_{11}(z)=$ $-\frac{i \sqrt{2}}{(5+k)} \sum_{(m, a)=(1,10)}^{(3,12)}\left(K^{m} \bar{D} J^{a}-\bar{D} K^{m} J^{a}\right)(z) \quad+\quad \frac{(3-i \sqrt{3})}{3 \sqrt{2}(5+k)}\left(K^{7} \bar{D} J^{9}-\bar{D} K^{7} J^{9}\right)(z) \quad+$ $\frac{(\sqrt{3}-3 i \sqrt{5})}{6(5+k)}\left(K^{8} \bar{D} J^{9}-\bar{D} K^{8} J^{9}\right)(z)+\frac{4 i \sqrt{2}}{(5+k)} \partial J^{9}(z)$.

${ }^{18}$ Furthermore, the constraint (2.7) implies that the current (2.12) can be written in terms of $G_{22}(z)=-\frac{i \sqrt{2}}{(5+k)} \sum_{(\bar{m}, \bar{a})=(\overline{1}, \overline{10})}^{(\overline{3}, \overline{1})}\left(K^{\bar{m}} D J^{\bar{a}}-D K^{\bar{m}} J^{\bar{a}}\right)(z)-\frac{(3+i \sqrt{3})}{3 \sqrt{2}(5+k)}\left(K^{\overline{7}} D J^{\overline{9}}-D K^{\overline{7}} J^{\overline{9}}\right)(z)-$ $\frac{(\sqrt{3}+3 i \sqrt{5})}{6(5+k)}\left(K^{\overline{8}} D J^{\overline{9}}-D K^{\overline{8}} J^{\overline{9}}\right)(z)-\frac{4 i \sqrt{2}}{(5+k)} \partial J^{\overline{9}}(z)$.
} 
Furthermore one obtains

$$
A_{i}(z) A_{j}(w)=\frac{1}{(z-w)^{2}}(-1) \frac{1}{2}(k+1) \delta_{i j}+\frac{1}{(z-w)} \epsilon_{i j k} A_{k}(w)+\cdots, \epsilon_{123}=1 .
$$

Therefore, the OPEs (2.14), (2.15), and (2.17) provide the $\mathrm{SU}(2)_{k^{+}}$current algebra of $\mathcal{N}=4$ large superconformal algebra. That is, the OPEs (2.17) coincide with the OPE $A^{+i}(z) A^{+j}(w)$ appearing in (C.3) of [39].

\subsubsection{Construction of four spin- $\frac{1}{2}$ currents and other two spin- $\frac{3}{2}$ currents}

How does one determine the four spin- $\frac{1}{2}$ currents appearing in (2.10)? Let us compute the OPE $A_{1}(z) G_{11}(w)$ explicitly. It turns out, from (2.13) and (2.11), that

$$
A_{1}(z) G_{11}(w)=\frac{1}{(z-w)^{2}} \frac{i}{2} 2(1-\gamma) F_{21}(w)-\frac{1}{(z-w)} \frac{i}{2} G_{21}(w)+\cdots,
$$

where the parameter is introduced

$$
\gamma=\frac{k^{-}}{\left(k^{+}+k^{-}\right)}=\frac{4}{(5+k)}
$$

and the new spin- $\frac{1}{2}$ current and the spin- $\frac{3}{2}$ current are

$$
\begin{aligned}
F_{21}(w)= & -\frac{(3 i+\sqrt{3})}{6 \sqrt{2}} K^{7}(w)-\frac{1}{12} i(\sqrt{3}-3 i \sqrt{5}) K^{8}(w) \\
G_{21}(w)= & \frac{\sqrt{2}}{5+k} \sum_{(a, \bar{a})=(9, \overline{9})}^{(12, \overline{12})} J^{a} D J^{\bar{a}}(w) \\
& + \text { other cubic and quadratic terms. }
\end{aligned}
$$

The OPE (2.18) corresponds to the OPE $A^{+i}(z) G_{a}(w)$ in $(C .3)$ of [39]. ${ }^{19}$

Let us consider the OPE $A_{1}(z) G_{22}(w)$. It turns out, from (2.13) and (2.12) together with (2.19), that

$$
A_{1}(z) G_{22}(w)=-\frac{1}{(z-w)^{2}} \frac{i}{2} 2(1-\gamma) F_{12}(w)+\frac{1}{(z-w)} \frac{i}{2} G_{12}(w)+\cdots,
$$

where ${ }^{20}$ one can read off the following currents

$$
\begin{aligned}
F_{12}(w)= & -\frac{(-3 i+\sqrt{3})}{6 \sqrt{2}} K^{\overline{7}}(w)+\frac{1}{12} i(\sqrt{3}+3 i \sqrt{5}) K^{\overline{8}}(w), \\
G_{12}(w)= & -\frac{\sqrt{2}}{5+k} \sum_{(a, \bar{a})=(9, \overline{9})}^{(12, \overline{12})} \bar{D} J^{a} J^{\bar{a}}(w) \\
& + \text { other cubic, quadratic and linear terms. }
\end{aligned}
$$

Again, this OPE (2.22) corresponds to the OPE $A^{+i}(z) G_{a}(w)$ in (C.3) of [39].

\footnotetext{
${ }^{19}$ More explicitly, their quantity $\alpha_{a}^{+i b}$ appearing in the right hand side of this OPE, in this particular case, becomes $\alpha_{a}^{+i b}=\alpha_{+K}^{+1-}=\delta^{-c} \alpha_{+K, c}^{+1}=2 \alpha_{+K,+}^{+1}$ which is equal to $2 \times \frac{i}{4}=\frac{i}{2}$. Here the index $a$ stands for $+K$, the index $b$ can be,,$+-+K$, or $-K$ and the index $i$ stands for 1 . Their nonzero $\delta_{a b}$ are given by $\delta_{+-}=\delta_{-+}=\delta_{+K,-K}=\delta_{-K,+K}=\frac{1}{2}($ not 1$)$. Similarly, $\delta^{+-}=\delta^{-+}=\delta^{+K,-K}=\delta^{-K,+K}=2($ not 1$)$. The quantity $\alpha_{a b}^{+i}$ are antisymmetric under $a \leftrightarrow b$. The 12 nonzero independent quantity $\alpha_{a b}^{ \pm i}$ can be obtained from the table 3 of [39].

${ }^{20}$ The quantity $\alpha_{a}^{+i b}$ in [39] leads to $\alpha_{-K,-}^{+1}=\frac{i}{4}$ and the normalization in the first-order pole in (2.22) is fixed.
} 
How does one determine the remaining spin- $\frac{1}{2}$ currents? Let us consider the following OPE $A_{3}(z) G_{11}(w)$. It turns out, from (2.16) and (2.11), that

$$
A_{3}(z) G_{11}(w)=\frac{1}{(z-w)^{2}} \frac{i}{2} 2(1-\gamma) F_{11}(w)-\frac{1}{(z-w)} \frac{i}{2} G_{11}(w)+\cdots,
$$

where $^{21}$ the spin- $\frac{1}{2}$ current is given by

$$
F_{11}(w)=\frac{i}{\sqrt{2}} J^{9}(w)
$$

Furthermore, the following OPE, which can be obtained from (2.16) and (2.12), provides other spin- $\frac{1}{2}$ current

$$
A_{3}(z) G_{22}(w)=-\frac{1}{(z-w)^{2}} \frac{i}{2} 2(1-\gamma) F_{22}(w)+\frac{1}{(z-w)} \frac{i}{2} G_{22}(w)+\cdots,
$$

where ${ }^{22}$ the spin- $\frac{1}{2}$ current is

$$
F_{22}(w)=-\frac{i}{\sqrt{2}} J^{\overline{9}}(w)
$$

The nontrivial OPEs from (2.20), (2.23), (2.26) and (2.28) are

$$
\begin{aligned}
& F_{11}(z) F_{22}(w)=-\frac{1}{(z-w)} \frac{1}{2} \frac{c}{6 \gamma(1-\gamma)}+\cdots, \\
& F_{12}(z) F_{21}(w)=-\frac{1}{(z-w)} \frac{1}{2} \frac{c}{6 \gamma(1-\gamma)}+\cdots .
\end{aligned}
$$

\subsubsection{Construction of other three spin-1 currents}

Let us continue to determine the remaining spin- 1 currents. From the OPEs $F_{11}(z) G_{21}(w)$ and $F_{12}(z) G_{22}(w)$, one obtains

$$
\begin{aligned}
& F_{11}(z) G_{21}(w)=\frac{1}{(z-w)}\left(-i B_{1}-B_{2}\right)(w)+\cdots, \\
& F_{12}(z) G_{22}(w)=\frac{1}{(z-w)}\left(-i B_{1}+B_{2}\right)(w)+\cdots,
\end{aligned}
$$

where the two new spin- 1 currents are ${ }^{23}$ obtained from the first-order poles in $(2.30)$

$$
\begin{aligned}
& B_{1}(w)=\frac{1}{2(5+k)} \sum_{(m, a)=(1,10)}^{(3,12)} K^{m} J^{a}(w)+\text { other quadratic terms, } \\
& B_{2}(w)=\frac{i}{2(5+k)} \sum_{(m, a)=(1,10)}^{(3,12)} K^{m} J^{a}(w)+\text { other quadratic terms. }
\end{aligned}
$$

\footnotetext{
${ }^{21}$ In this case, the quantity $\alpha_{a}^{+i b}$ becomes $\alpha_{+K,-K}^{+3}=-\frac{i}{4}$ which agrees with the result of [39].

${ }^{22}$ Furthermore, one has consistent expression $\alpha_{-K,+K}^{+3}=\frac{i}{4}$.

${ }^{23}$ One obtains the quantities $\alpha_{+K,-}^{-1}=\frac{i}{4}$ and $\alpha_{+K,-}^{-2}=\frac{1}{4}$. Similarly one uses $\alpha_{+,-K}^{-1}=\frac{i}{4}$ and $\alpha_{+,-K}^{-2}=-\frac{1}{4}$. All these results are consistent with the results in [39].
} 
Now one obtains the third spin-1 current from (2.31) as follows:

$$
B_{1}(z) B_{2}(w)=\frac{1}{(z-w)} B_{3}(w)+\cdots
$$

where the spin-1 current can be read off

$$
B_{3}(w)=-\frac{i}{2(5+k)} \sum_{(a, \bar{a})=(9, \overline{9})}^{(12, \overline{2})} J^{a} J^{\bar{a}}(w)+\text { other quadratic terms. }
$$

One obtains the following OPEs from (2.31) and (2.33)

$$
B_{i}(z) B_{j}(w)=-\frac{1}{(z-w)^{2}} 2 \delta_{i j}+\frac{1}{(z-w)} \epsilon_{i j k} B_{k}(w)+\cdots
$$

Therefore, the OPEs (2.32) and (2.34) provide the $\mathrm{SU}(2)_{k^{-}}$current algebra with $k^{-}=4$ of large $\mathcal{N}=4$ linear superconformal algebra. That is, the OPEs (2.34) coincide with the OPE $A^{-i}(z) A^{-j}(w)$ appearing in (C.3) of [39].

\subsubsection{Construction of other spin-1 current}

Finally, the undetermined spin- 1 current can be obtained from the OPE $F_{11}(z) G_{22}(w)$

$$
F_{11}(z) G_{22}(w)=\frac{1}{(z-w)}\left(-i A_{3}-i B_{3}+U\right)(w)+\cdots
$$

where ${ }^{24}$ the first-order pole with previous expressions for $A_{3}(w)$ and $B_{3}(w)$ in $(2.16)$ and (2.33) provides the information of $\mathrm{U}(w)$ and the spin-1 current is given by

$$
\begin{aligned}
\mathrm{U}(w)= & \frac{1}{12}(-3 i-\sqrt{3}) \bar{D} K^{7}(w)-\frac{(i \sqrt{3}+3 \sqrt{5})}{12 \sqrt{2}} \bar{D} K^{8}(w)+\frac{1}{12}(-3 i+\sqrt{3}) D K^{\overline{7}}(w) \\
& +\frac{(-i \sqrt{3}+3 \sqrt{5})}{12 \sqrt{2}} D K^{\overline{8}}(w) .
\end{aligned}
$$

From (2.36), the following OPE can be obtained

$$
\mathrm{U}(z) \mathrm{U}(w)=-\frac{1}{(z-w)^{2}} \frac{c}{12 \gamma(1-\gamma)}+\cdots
$$

Note that one can reexpress the spin- 1 currents using the superpartner of $F_{12}(z)$ and $F_{21}(z)$, i.e. $D F_{12}(z)$ and $\bar{D} F_{21}(z)$. Then one sees that

$$
\mathrm{U}(z)=\frac{1}{\sqrt{2}}\left(\bar{D} F_{21}-D F_{12}\right)(z)
$$

\footnotetext{
${ }^{24}$ In this case, one reads off $\alpha_{+K,-K}^{+3}=-\frac{i}{4}$ and $\alpha_{+K,-K}^{-3}=\frac{i}{4}$ which agree with the ones in [39].
} 


\subsubsection{Construction of spin-2 current}

One obtains the stress tensor as a difference between the stress tensor of the group SU(5) and the stress tensor of the subgroup SU(3):

$$
T(z)=T_{\mathrm{SU}(5)}(z)-T_{\mathrm{SU}(3)}(z) .
$$

Then one can obtain the following OPEs

$$
\begin{aligned}
T(z)_{\mathrm{SU}(5)} T(w)_{\mathrm{SU}(5)}= & \frac{1}{(z-w)^{4}} \frac{c_{\mathrm{SU}(5)}}{2}+\frac{1}{(z-w)^{2}} 2 T(w)_{\mathrm{SU}(5)}+\frac{1}{(z-w)} \partial T(w)_{\mathrm{SU}(5)}+\cdots, \\
T(z)_{\mathrm{SU}(5)} T(w)_{\mathrm{SU}(3)}= & \frac{1}{(z-w)^{4}} \frac{c_{\mathrm{SU}(3)}}{2}+\frac{1}{(z-w)^{2}} 2 T(w)_{\mathrm{SU}(3)}+\frac{1}{(z-w)} \partial T(w)_{\mathrm{SU}(3)}+\cdots, \\
T(z)_{\mathrm{SU}(3)} T(w)_{\mathrm{SU}(3)}= & \frac{1}{(z-w)^{4}} \frac{c_{\mathrm{SU}(3)}}{2}+\frac{1}{(z-w)^{2}} 2 T(w)_{\mathrm{SU}(3)}+\frac{1}{(z-w)} \partial T(w)_{\mathrm{SU}(3)} \\
& +\cdots,
\end{aligned}
$$

where the central charges are given by

$$
c_{\mathrm{SU}(5)}=\frac{12(5+3 k)}{(5+k)}, \quad c_{\mathrm{SU}(3)}=\frac{12(3+k)}{(5+k)}, \quad c=c_{\mathrm{SU}(5)}-c_{\mathrm{SU}(3)}=\frac{24(1+k)}{(5+k)} .
$$

Then the standard OPE between the stress tensor $T(z)$, that can be obtained from (2.40), has the following form

$$
T(z) T(w)=\frac{1}{(z-w)^{4}} \frac{c}{2}+\frac{1}{(z-w)^{2}} 2 T(w)+\frac{1}{(z-w)} \partial T(w)+\cdots
$$

where the central charge is given in (2.41).

One can easily check that there are no singular terms in the OPEs between the generators of the large $\mathcal{N}=4$ linear superconformal algebra and the subgroup stress tensor as follows: $:^{25}$

$$
T_{\mathrm{SU}(3)}(z) \Phi(w)=+\cdots,
$$

where $\Phi(w)$ is 16 currents of large $\mathcal{N}=4$ linear superconformal algebra. There are no singular terms in the OPEs between the generators of the large $\mathcal{N}=4$ linear superconformal algebra and the two subgroup spin- $\frac{3}{2}$ currents, $G_{12}^{\mathrm{SU}(3)}(z)$ and $G_{21}^{\mathrm{SU}(3)}(z) \cdot{ }^{26}$

\footnotetext{
${ }^{25}$ Instead, there exist the singular terms in the OPEs between 16 currents of large $\mathcal{N}=4$ linear algebra and $\mathcal{N}=2$ WZW affine currents (having the indices 4,5,6 and some linear combination of the index 7 and the index 8 and their conjugated ones) living in the subgroup SU(3). In next section, by factoring out spin-1 current and four spin- $\frac{1}{2}$ currents and modifying the remaining 11 currents correctly, the 11 currents of the large $\mathcal{N}=4$ nonlinear superconformal algebra commute with the SU(3) WZW affine currents. See also the footnote 53 .

${ }^{26}$ That is, $G_{12}^{\mathrm{SU}(3)}(z) \Phi(w)=+\cdots$ and $G_{21}^{\mathrm{SU}(3)}(z) \Phi(w)=+\cdots$, where $\Phi(w)$ is defined in (2.43).
} 
One can rearrange the 16 currents in terms of four $\mathcal{N}=2$ multiplets as follows:

$$
\begin{aligned}
& \left(1, \frac{3}{2}, \frac{3}{2}, 2\right):\left(-2 i \gamma A_{3}-2 i(1-\gamma) B_{3}, \quad G_{21}, \quad G_{12}, \quad T\right), \\
& \left(\frac{1}{2}, 1,1, \frac{3}{2}\right):\left(2 F_{11}, \quad 2 i\left(B_{1}-i B_{2}\right), \quad-i\left(A_{1}+i A_{2}\right), \quad G_{11}+(1-2 \gamma) \partial F_{11}\right), \\
& \left(\frac{1}{2}, 1,1, \frac{3}{2}\right):\left(2 F_{22}, \quad-2 i\left(A_{1}-i A_{2}\right), \quad i\left(B_{1}+i B_{2}\right), \quad-G_{22}+(1-2(1-\gamma)) \partial F_{22}\right), \\
& \left(0, \frac{1}{2}, \frac{1}{2}, 1\right):\left(2 \int U d z, \quad-2 F_{21}, \quad F_{12}, \quad-i\left(A_{3}-B_{3}\right)\right) .
\end{aligned}
$$

In the first $\mathcal{N}=2$ multiplet of (2.44), the OPE between $G_{21}(z)$ and $G_{12}(w)$ provides $\mathcal{N}=2$ $\mathrm{U}(1)$ current of spin-1 and the stress tensor. For given lowest component of second $\mathcal{N}=2$ multiplet in (2.44), the third component can be read off from the OPE between $G_{12}(z)$ and $F_{11}(w)$. Similarly, the second component can be obtained from the OPE between $G_{21}(z)$ and $F_{11}(w)$. The last component can be obtained from the OPE between $G_{12}(z)$ and $i\left(B_{1}-i B_{2}\right)(w) .{ }^{27}$ For given lowest component of third $\mathcal{N}=2$ multiplet in (2.44), the third component can be read off from the OPE between $G_{12}(z)$ and $F_{22}(w)$. Similarly, the second component can be obtained from the OPE between $G_{21}(z)$ and $F_{22}(w)$. The last component can be obtained from the OPE between $G_{12}(z)$ and $i\left(A_{1}-i A_{2}\right)(w) .{ }^{28}$

Therefore, the large $\mathcal{N}=4$ linear superconformal algebra is realized by the spin2 current, (2.39), the four spin- $\frac{3}{2}$ currents, (2.11), (2.12), (2.21) and (2.24), the spin-1 currents, (2.13), (2.16), (2.31), (2.33) and (2.36), the spin- $\frac{1}{2}$ currents, (2.20), (2.23), (2.26) and (2.28). Some of the algebra consist of (2.14), (2.15), (2.17), (2.18), (2.22), (2.25), (2.27), (2.29), (2.30), (2.32), (2.34), (2.35), (2.37) and (2.42). The remaining OPEs can be obtained similarly. ${ }^{29}$

\section{The large $\mathcal{N}=4$ nonlinear superconformal algebra in the Wolf space coset minimal model}

The large $\mathcal{N}=4$ nonlinear algebra can be obtained from the linear one described in previous section by factoring out the four fermions and a spin-1 bosonic current. ${ }^{30}$

\footnotetext{
${ }^{27}$ In other words, one has the following OPEs: $G_{21}(z) F_{11}(w)=-\frac{1}{(z-w)} i\left(B_{1}-i B_{2}\right)(w)+\cdots$, $G_{12}(z) F_{11}(w)=-\frac{1}{(z-w)} i\left(A_{1}+i A_{2}\right)(w)+\cdots$, and $G_{12}(z) i\left(B_{1}-i B_{2}\right)(w)=-\frac{1}{(z-w)^{2}} \frac{8}{(5+k)} F_{11}(w)+$ $\frac{1}{(z-w)}\left[-\frac{8}{(5+k)} \partial F_{11}+G_{11}\right](w)+\cdots$.

${ }^{28}$ Similarly one has $G_{21}(z) F_{22}(w)=-\frac{1}{(z-w)} i\left(A_{1}-i A_{2}\right)(w)+\cdots, \quad G_{12}(z) F_{22}(w)=$ $\frac{1}{(z-w)} i\left(B_{1}+i B_{2}\right)(w)+\cdots \quad$ and $\quad G_{12}(z) i\left(A_{1}-i A_{2}\right)(w) \quad=\quad \frac{1}{(z-w)^{2}} \frac{2(1+k)}{(5+k)} F_{22}(w)+$ $\frac{1}{(z-w)}\left[\frac{2(1+k)}{(5+k)} \partial F_{11}+G_{22}\right](w)+\cdots$.

${ }^{29}$ There exists the middle $\mathcal{N}=4$ linear superconformal algebra where $\mathrm{SU}(2) \times \mathrm{U}(1)^{4}$ affine algebra is present in [40].

${ }^{30}$ The main reason why we should decouple these five currents is that they do not appear in the $D\left(2,1 \mid \frac{\mu}{1-\mu}\right)$ wedge subalgebra and therefore also in the higher spin theory of [1]. In the CFT computations characterized by any OPEs obtained in this paper, because the number of independent currents is reduced from 16 to 11 , there are no computational complications in the calculations of the OPE. In particular, we do not have to consider any spin- $\frac{1}{2}$ currents in the composite fields of any spin appearing in the singular terms of the OPE.
} 


\subsection{Construction of spin-2 stress tensor}

The additional terms in the stress energy tensor of spin- 2 consist of the quadratic term of spin-1 current and the 16 quadratic terms of spin- $\frac{1}{2}$ currents with derivative. Then the total 19 unknown coefficient functions can be determined from the following regular conditions between the $\mathrm{U}(z), F_{11}(z), F_{12}(z), F_{21}(z)$ and $F_{22}(z)$ and the stress energy tensor $\hat{T}=T+T_{\bmod }[7,41,42]$ :

$$
\mathrm{U}(z) \hat{T}(w)=+\cdots, \quad F_{a}(z) \hat{T}(w)=+\cdots, \quad a=11,12,21,22 .
$$

For $N=3$, the stress energy tensor of the large $\mathcal{N}=4$ nonlinear algebra can be written as $[7,41,42]$

$$
\begin{aligned}
\hat{T}(z) & =T(z)+\frac{1}{(5+k)} U \mathrm{U}(z)+\frac{1}{(5+k)}\left(\partial F_{11} F_{22}+\partial F_{12} F_{21}+\partial F_{21} F_{12}+\partial F_{22} F_{11}\right)(z) \\
& =T_{\mathrm{SU}(5)}(z)-T_{\mathrm{SU}(3)}(z)+\frac{1}{(5+k)}\left(U U+\partial F^{a} F_{a}\right)(z) .
\end{aligned}
$$

The general $N$ case can be obtained by putting $(5+k) \rightarrow(k+N+2)$ and $T \rightarrow T_{\mathrm{SU}(N+2)}-$ $T_{\mathrm{SU}(N)}$ in $(3.2)$ similarly. One can read off the corresponding central charge appearing in the Virasoro algebra by calculating the OPE $\hat{T}(z) \hat{T}(w) \cdot{ }^{31}$ Therefore, the total central charge is given by

$$
\hat{c}=\frac{6(k+1)(N+1)}{(k+N+2)}-2-4+1+2=\frac{3(k+N+2 k N)}{(k+N+2)} \rightarrow \frac{3(3+7 k)}{(5+k)},
$$

where the $N=3$ is substituted in the last stage.

\subsection{Construction of six spin-1 currents}

Let us determine the other currents. For the spin- 1 current, one can add 6 additional terms coming from four fermions, $F_{11}(z), F_{12}(z), F_{21}(z)$ and $F_{22}(z)$. The relative coefficient functions, as done in (3.1), can be determined by the regular conditions [7, 41, 42]

$$
\mathrm{U}(z) \hat{A}_{1}(w)=+\cdots, \quad F_{a}(z) \hat{A}_{1}(w)=+\cdots, \quad a=11,12,21,22 .
$$

It turns out that the spin- 1 current is given by

$$
\hat{A}_{1}(z)=A_{1}(z)-\frac{1}{(5+k)}\left(\frac{i}{2} F_{11} F_{12}-\frac{i}{2} F_{12} F_{11}+\frac{i}{2} F_{21} F_{22}-\frac{i}{2} F_{22} F_{21}\right)(z) .
$$

One can further simplify (3.5) using the fact that $F_{11} F_{12}(z)=-F_{12} F_{11}(z)$ and $F_{21} F_{22}(z)=$ $-F_{22} F_{21}(z)$.

\footnotetext{
${ }^{31}$ The OPE between $U \mathrm{U}(z)$ term and itself contributes to 1 for the central charge while the OPEs between the fermions and itself contribute to 2. The OPE between $T(z)$ and the $U \mathrm{U}(w)$ (and the OPE between $U \mathrm{U}(z)$ and $T(w))$ contributes -2 and similarly the contributions from the $T(z)$ and fermion terms give to -4 . This can be seen from the $(2.3)$ by realizing that the central term coming from the three fermions in $\mathrm{SU}(2)$ is given by $\frac{1}{2} \times 3=\frac{3}{2}$ while the one from the spin- 1 and spin- $\frac{1}{2}$ fields in U(1) is given by $\frac{3}{2}$. Therefore, the sum of these is equal to 3 .
} 
Similarly, the other currents of spin-1 can be obtained as follows:

$$
\hat{A}_{i}(z)=A_{i}(z)-\frac{1}{(5+k)} \alpha_{a b}^{+i} F^{a} F^{b}, \quad i=2,3, \quad a, b=11,12,21,22 .
$$

It is straightforward to calculate the following OPEs from (3.5) and (3.6)

$$
\hat{A}_{i}(z) \hat{A}_{j}(w)=-\frac{1}{(z-w)^{2}} \frac{1}{2} \hat{k}^{+} \hat{\delta}_{i j}+\frac{1}{(z-w)} \epsilon_{i j k} \hat{A}_{k}(w)+\cdots, \quad \hat{\delta}_{i i}=1,
$$

where the new level is given by $\hat{k}^{+}=k^{+}-1=k$. The three quadratic terms appearing in (3.5) and (3.6) satisfy the $\mathrm{SU}(2)_{1}$ current algebra of (3.18) in [1]. It is obvious that the OPEs between these three currents and $\hat{A}_{1}(z), \hat{A}_{2}(z)$ and $\hat{A}_{3}(z)$ (that correspond to $\hat{J}(z)$ around the equation (3.18) in [1]) do not have the singular terms due to the conditions $[7,41,42]$ like as $(3.4)$ :

$$
\mathrm{U}(z) \hat{A}_{i}(w)=+\cdots, \quad F_{a}(z) \hat{A}_{i}(w)=+\cdots, \quad i=2,3, \quad a=11,12,21,22 .(3.8)
$$

Similarly, the currents $A_{1}(z), A_{2}(z)$ and $A_{3}(z)$ correspond to $J(z)$ appearing around the equation (3.18) of [1]. ${ }^{32}$ Compared to (2.13) and (2.16), the currents $\hat{A}_{1}(z)$ and $\hat{A}_{2}(z)$ contain the quadratic parts in the fermions while the quadratic parts in the fermions with the indices 7 and 8 appearing in $A_{3}(z)$ disappear in the current $\hat{A}_{3}(z) .{ }^{33}$

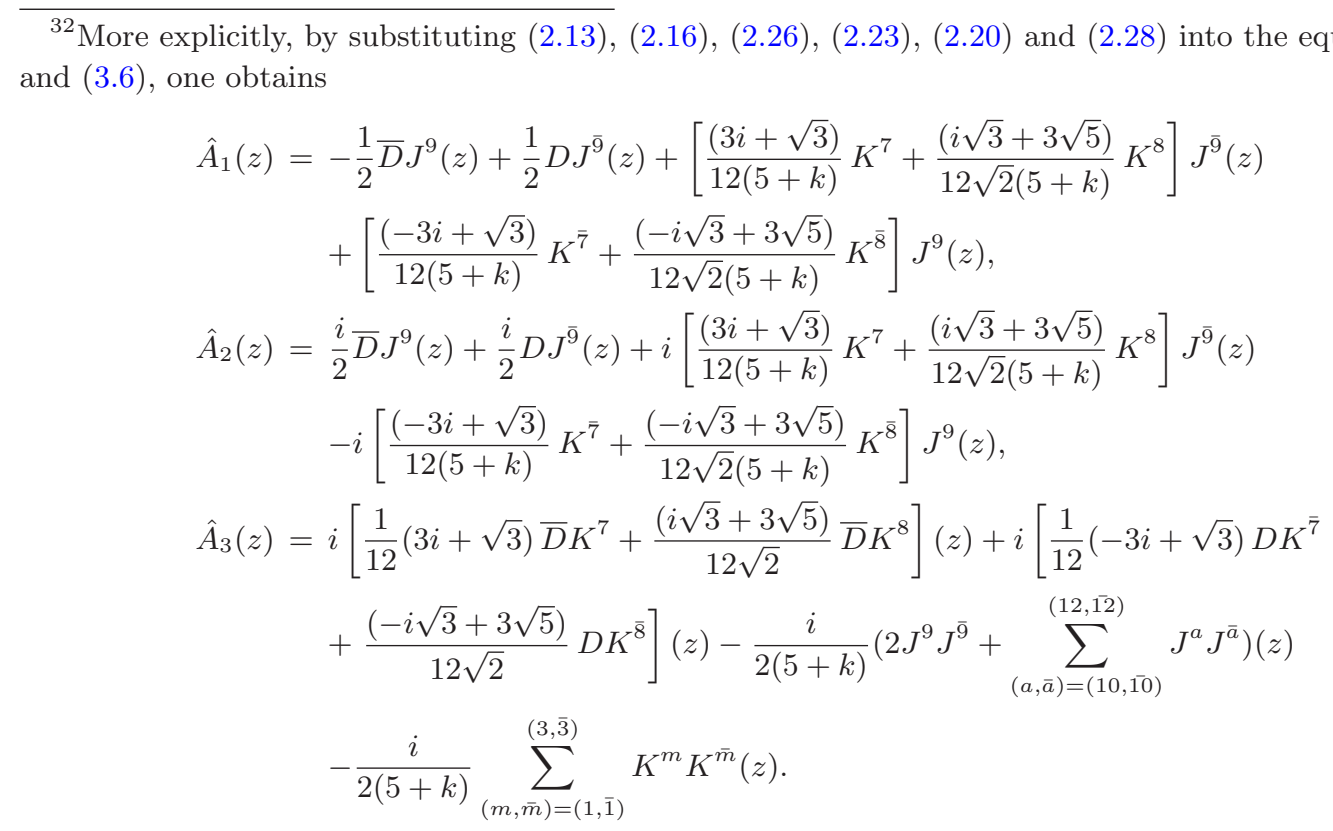

${ }^{33}$ As observed in (2.13) and (2.16), the currents $A_{1}(z), A_{2}(z)$ and $A_{3}(z)$ contain the linear spin-1 currents (not quadratic fermions). Then one knows its superpartners, $J^{9}(z), J^{\overline{9}}(z)$ and a linear combination between $K^{7}(z), K^{8}(z), K^{\overline{7}}(z)$ and $K^{\overline{8}}(z)$. These are the three fermions in $s u(2)_{\kappa}^{(1)}$ of [1]. Note that from $(2.31)$ and (2.33) there are no linear spin-1 currents. All of the expressions are written in terms of quadratic fermions. Note that $\hat{A}_{1}(z)$ and $\hat{A}_{2}(z)$ contain the Wolf space subgroup indices in their expressions while the $\hat{A}_{3}(z)$ contains both Wolf space subgroup indices and Wolf space coset indices. Therefore, the $\mathrm{SU}(2)_{k}$ algebra of large $\mathcal{N}=4$ nonlinear algebra comes from two currents from the Wolf space subgroup and one current from both the subgroup and the coset of Wolf space. Note that the $\mathrm{SU}(2)_{k+1}$ algebra of large $\mathcal{N}=4$ linear algebra comes from three currents from the coset $\frac{\mathrm{SU}(5)}{\mathrm{SU}(3)}$. This is consistent with the equation (3.7) of $[15]$. 
Let us move on the other type of spin-1 currents. As done before, the spin-1 currents, $B_{1}(z), B_{2}(z)$ and $B_{3}(z)$ (that correspond to $K(z)$ appearing around the equation (3.18) of [1]) can be modified under the factoring out the fermions and spin-1 current. One obtains $[7,41,42]$

$$
\hat{B}_{i}(z)=B_{i}(z)-\frac{1}{(5+k)} \alpha_{a b}^{-i} F^{a} F^{b}, \quad i=1,2,3, \quad a, b=11,12,21,22 .
$$

Their current algebra can be summarized by

$$
\hat{B}_{i}(z) \hat{B}_{j}(w)=-\frac{1}{(z-w)^{2}} \frac{1}{2} \hat{k}^{-} \hat{\delta}_{i j}+\frac{1}{(z-w)} \epsilon_{i j k} \hat{B}_{k}(w)+\cdots,
$$

where the new level $\hat{k}^{-}=k^{-}-1=N=3$. Three of four in $\tilde{K}^{\alpha \beta}(z)$ defined in the equation (3.14) [1] correspond to the present $\hat{B}_{1}(z), \hat{B}_{2}(z)$ and $\hat{B}_{3}(z)$. The three quadratic terms appearing in (3.10) satisfy the other $\mathrm{SU}(2)_{1}$ current algebra of (3.18) in [1] and therefore the OPEs between these three currents and the three currents appearing in (3.5) and (3.6) (satisfying other $\mathrm{SU}(2)_{1}$ current algebra) do not have the singular terms. ${ }^{34}$ Compared to (2.31) and (2.33), the quadratic parts having the indices 7,8 or 9 disappear in (3.12). ${ }^{35}$

\subsection{Construction of four spin- $\frac{3}{2}$ currents}

One can construct four spin- $\frac{3}{2}$ currents $[7,41,42]$

$$
\begin{aligned}
\hat{G}_{a}(z)= & G_{a}(z)+\frac{2}{(5+k)} U F_{a}(z)-\frac{2}{3(5+k)^{2}} \epsilon_{a b c d} F^{b} F^{c} F^{d}(z) \\
& +\frac{4}{(5+k)} F^{b}\left(\alpha_{b a}^{+i} \hat{A}_{i}-\alpha_{b a}^{-i} \hat{B}_{i}\right), \quad i=1,2,3, \quad a, b, \cdots=11,12,21,22(3.13)
\end{aligned}
$$

The expressions in (3.21) of [1] correspond to the extra fields in (3.13). The relative coefficient functions in (3.13) are determined completely by requiring that they should commute with $\mathrm{U}(1)$ current and four fermions of spin- $\frac{1}{2} \cdot 36$

\footnotetext{
${ }^{34}$ Furthermore, by substituting (2.31), (2.33), (2.26), (2.23), (2.20) and (2.28) into the equations (3.10), one has

$$
\begin{aligned}
& \hat{B}_{1}(z)=\frac{1}{2(5+k)}\left(\sum_{(m, a)=(1,10)}^{(3,12)} K^{m} J^{a}+\sum_{(\bar{m}, \bar{a})=(\overline{1}, \overline{10})}^{(\overline{3}, \overline{1} 2)} K^{\bar{m}} J^{\bar{a}}\right)(z), \\
& \hat{B}_{2}(z)=\frac{i}{2(5+k)}\left(\sum_{(m, a)=(1,10)}^{(3,12)} K^{m} J^{a}-\sum_{(\bar{m}, \bar{a})=(\overline{1}, \overline{10})}^{(3,1 \overline{2})} K^{\bar{m}} J^{\bar{a}}\right)(z), \\
& \hat{B}_{3}(z)=-\frac{i}{2(5+k)}\left(\sum_{(a, \bar{a})=(10, \overline{10})}^{(12, \overline{1} 2)} J^{a} J^{\bar{a}}+\sum_{(m, \bar{m})=(1, \overline{1})}^{(3, \overline{3})} K^{m} K^{\bar{m}}\right)(z) .
\end{aligned}
$$

${ }^{35}$ Note that all the currents $\hat{B}_{1}(z), \hat{B}_{2}(z)$ and $\hat{B}_{3}(z)$ contain the Wolf space coset indices only. Therefore the $\mathrm{SU}(2)_{3}$ algebra of large $\mathcal{N}=4$ nonlinear superconformal algebra comes from the currents in the Wolf space coset. This is consistent with the result for the structure of $J^{M-}$ in (3.18) of [24]. Recall that the $\mathrm{SU}(2)_{4}$ algebra of large $\mathcal{N}=4$ linear superconformal algebra comes from the currents in the coset $\frac{\mathrm{SU}(5)}{\mathrm{SU}(3)}$. This is consistent with the equation (3.7) of [15]. The spin-1 currents in (3.12) satisfy the following regular conditions, as in (3.8), as follows: $\mathrm{U}(z) \hat{B}_{i}(w)=+\cdots$ and $F_{a}(z) \hat{B}_{i}(w)=+\cdots$.

${ }^{36}$ In other words, $\mathrm{U}(z) \hat{G}_{a}(w)=+\cdots$ and $F_{a}(z) \hat{G}_{b}(w)=+\cdots$.
} 
By substituting the expressions (2.26), (2.23), (2.20), (2.28), (2.36), (2.11), (2.24), (2.21), (2.12), (3.9) and (3.10) into the equations (3.13), the following results can be obtained

$$
\begin{aligned}
\hat{G}_{11}(z)= & -\frac{i \sqrt{2}}{(5+k)} \sum_{(m, a)=(1,10)}^{(3,12)}\left(K^{m} \bar{D} J^{a}-\bar{D} K^{m} J^{a}\right)(z) \\
& + \text { other cubic and linear terms, } \\
\hat{G}_{22}(z)= & -\frac{i \sqrt{2}}{(5+k)} \sum_{(\bar{m}, \bar{a})=(\overline{1}, \overline{10})}^{(\overline{3}, \overline{12})}\left(K^{\bar{m}} D J^{\bar{a}}-D K^{\bar{m}} J^{\bar{a}}\right)(z) \\
& + \text { other cubic and linear terms, } \\
\hat{G}_{12}(z)= & -\frac{\sqrt{2}}{(5+k)} \sum_{(a, \bar{a})=(10, \overline{10})}^{(12, \overline{1})} \bar{D} J^{a} J^{\bar{a}}(z) \\
& + \text { other cubic, quadratic and linear terms, } \\
\hat{G}_{21}(z)= & \frac{\sqrt{2}}{(5+k)} \sum_{(a, \bar{a})=(10, \overline{1})}^{(12, \overline{1})} J^{a} D J^{\bar{a}}(z) \\
& + \text { other cubic and quadratic terms. }
\end{aligned}
$$

The quadratic parts having the indices 7,8 , or 9 in $G_{11}(z)$ disappear in $\hat{G}_{11}(z)$ and similarly, those having the indices $\overline{7}, \overline{8}$, or $\overline{9}$ in $G_{22}(z)$ disappear in $\hat{G}_{22}(z)$. Furthermore, the quadratic parts having the indices 7,8 , or 9 (and $\overline{7}, \overline{8}$ or $\overline{9}$ ) in $G_{12}(z)$ disappear in $\hat{G}_{12}(z)$. The cubic terms containing the indices $9, \overline{9}$ in $G_{12}(z)$ disappear. Similarly, the quadratic parts having the indices 7,8 , or 9 (and $\overline{7}, \overline{8}$ or $\overline{9}$ ) in $G_{21}(z)$ disappear in $\hat{G}_{21}(z)$. The cubic terms containing the indices $9, \overline{9}$ in $G_{21}(z)$ disappear in $\hat{G}_{21}(z) .{ }^{37}$

The full algebra can be obtained from the OPEs between 11 currents. For example, from the explicit expressions in (3.16) and (3.17), the following OPE can be described as

$$
\begin{aligned}
\hat{G}_{12}(z) \hat{G}_{21}(w)= & \frac{1}{(z-w)^{3}} \frac{2}{3} c_{\text {Wolf }}+\frac{1}{(z-w)^{2}}\left[4 i \gamma_{A} \hat{A}_{3}+4 i \gamma_{B} \hat{B}_{3}\right](w) \\
& +\frac{1}{(z-w)}\left[2 \hat{T}+\frac{1}{2} 4 i \gamma_{A} \partial \hat{A}_{3}+\frac{1}{2} 4 i \gamma_{B} \partial \hat{B}_{3}+\right. \\
& \left.+\frac{2}{(5+k)}\left(\sum_{i=1}^{3} \hat{A}_{i} \hat{A}_{i}-2 \hat{A}_{3} \hat{B}_{3}+\sum_{i=1}^{3} \hat{B}_{i} \hat{B}_{i}\right)\right](w) \\
& +\cdots
\end{aligned}
$$

\footnotetext{
${ }^{37}$ For the $\hat{G}_{11}(z)$ and $\hat{G}_{22}(z)$, the terms having the indices $\overline{7}$ and $\overline{8}$ can be rewritten using $F_{12}(z)(2.23)$ and the terms having the indices 7 and 8 can be expressed using the $F_{21}(z)(2.20)$. The $F_{12}(z)$ and $F_{21}(z)$ are currents of $\mathrm{SU}(2)$ and $\mathrm{U}(1)$ factors in the denominator of the Wolf space coset (2.1). See also the footnote 42 . However, for $\hat{G}_{12}(z)$ and $\hat{G}_{21}(z)$, it is not obvious to see whether the terms including the indices $7,8, \overline{7}$ and $\overline{8}$ can be rewritten in terms of the currents with indices of the subgroup of the Wolf space coset. Because the currents $K^{7}(z), K^{\overline{7}}(z), K^{8}(z)$ and $K^{\overline{8}}(\mathrm{z})$ can be expressed in terms of two $\mathrm{SU}(3)$ currents, one $\mathrm{SU}(2)$ current and one $\mathrm{U}(1)$ current, one realizes that any spin- $\frac{1}{2}$ fermion terms including the indices $7,8, \overline{7}$ and $\overline{8}$ have the subgroup $\mathrm{SU}(3), \mathrm{SU}(2)$ or $\mathrm{U}(1)$ indices in the Wolf space coset. See also the footnote 53 . All the spin- $\frac{3}{2}$ currents have both subgroup indices and coset indices of Wolf space.
} 
where the central charge in the highest pole of this OPE (3.18) is $c_{\text {Wolf }}=\frac{18 k}{(5+k)}$ which coincides with (2.3) for $N=3$. One can easily check that the central terms from the extra three OPEs coming from the left hand side of (3.18) except $G_{12}(z) G_{21}(w)$ can be written as $-\frac{6(4+k)}{(5+k)}$ which is exactly the same as the contributions from $\mathrm{SU}(2)$ and $\mathrm{U}(1)$ in $(2.3)$. The parameters $\gamma_{A}$ and $\gamma_{B}$ are given by $\gamma_{A}=\frac{\hat{k}^{-}}{\hat{k}^{+}+\hat{k}^{-}+2}=\frac{3}{(5+k)}$ and $\gamma_{B}=\frac{\hat{k}^{+}}{\hat{k}^{+}+\hat{k}^{-}+2}=\frac{k}{(5+k)}$. The stress tensor $\hat{T}(z)$ is given by (3.2). Compared to the OPE $G_{12}(z) G_{21}(w)$ described in previous section, the nonlinear terms occur in the first-order pole in (3.18). ${ }^{38}$ This is due to the fact that the OPEs between the extra terms in (3.13) contribute to these nonlinear terms in the right hand side of (3.18).

\section{$3.4 \quad \mathrm{U}(1)$ charge}

As in $\mathrm{U}(1)$ current of (2.44), one can describe the $\mathrm{U}(1)$ current here. By realizing that the $\mathrm{U}(1)$ current appears in the second-order pole of the OPE $\hat{G}_{21}(z) \hat{G}_{12}(w)$, the U(1) current is identified with $\left(-2 i \gamma_{A} \hat{A}_{3}-2 i \gamma_{B} \hat{B}_{3}\right)(z)$ from the OPE (3.18). Then one obtains the following first-order poles for the 11 currents as follows:

$$
\begin{aligned}
&\left.\left(-2 i \gamma_{A} \hat{A}_{3}-2 i \gamma_{B} \hat{B}_{3}\right)(z) \hat{A}_{ \pm}(w)\right|_{\frac{1}{(z-w)}}=\frac{1}{(z-w)}\left[\mp \frac{6}{(5+k)}\right] \hat{A}_{ \pm}(w) \\
&\left.\left(-2 i \gamma_{A} \hat{A}_{3}-2 i \gamma_{B} \hat{B}_{3}\right)(z) \hat{B}_{ \pm}(w)\right|_{\frac{1}{(z-w)}}=\frac{1}{(z-w)}\left[\mp \frac{2 k}{(5+k)}\right] \hat{B}_{ \pm}(w) \\
&\left.\left(-2 i \gamma_{A} \hat{A}_{3}-2 i \gamma_{B} \hat{B}_{3}\right)(z) \hat{A}_{3}(w)\right|_{\frac{1}{(z-w)}}=0 \\
&\left.\left(-2 i \gamma_{A} \hat{A}_{3}-2 i \gamma_{B} \hat{B}_{3}\right)(z) \hat{B}_{3}(w)\right|_{\frac{1}{(z-w)}}=0 \\
&\left.\left(-2 i \gamma_{A} \hat{A}_{3}-2 i \gamma_{B} \hat{B}_{3}\right)(z)\left(\begin{array}{l}
\hat{G}_{11} \\
\hat{G}_{22}
\end{array}\right)(w)\right|_{\frac{1}{(z-w)}}=\frac{1}{(z-w)}\left[ \pm \frac{(-3+k)}{(5+k)}\right]\left(\begin{array}{c}
\hat{G}_{11} \\
\hat{G}_{22}
\end{array}\right)(w), \\
&\left.\left(-2 i \gamma_{A} \hat{A}_{3}-2 i \gamma_{B} \hat{B}_{3}\right)(z)\left(\begin{array}{l}
\hat{G}_{12} \\
\hat{G}_{21}
\end{array}\right)(w)\right|_{\frac{1}{(z-w)}}=\frac{1}{(z-w)}\left[\mp \frac{(3+k)}{(5+k)}\right]\left(\begin{array}{c}
\hat{G}_{12} \\
\hat{G}_{21}
\end{array}\right)(w) \\
&\left.\left(-2 i \gamma_{A} \hat{A}_{3}-2 i \gamma_{B} \hat{B}_{3}\right)(z) \hat{T}(w)\right|_{\frac{1}{(z-w)}}=0 .
\end{aligned}
$$

For the nonzero U(1) charges, we present the correspoding two expressions together in order to emphasize the fact that they have opposite $\mathrm{U}(1)$ charges. ${ }^{39}$ We present the $\mathrm{U}(1)$ charges for those currents in the table 1 . This definite $\mathrm{U}(1)$ charges will play an important role in any OPEs in this paper because the U(1) charge conservation holds for any OPEs. For example, in the OPE between the spin- $\frac{3}{2}$ current and the spin-3 current (the most complicated OPE in this paper), the first-order pole contains the spin- $\frac{7}{2}$ field. Without the $\mathrm{U}(1)$ charge conservation, in general, the possible spin- $\frac{7}{2}$ field has too many terms.

\footnotetext{
${ }^{38}$ Using the following values $\alpha_{+,-}^{+3}=-\frac{i}{4}, \alpha_{+,+K}^{+2}=-\frac{1}{4}$ and $\alpha_{-,-K}^{+2}=-\frac{1}{4}$ (therefore, we present all the 12 independent quantities with previous footnotes), this particular OPE coincides with (A.7) of [42]. Note that our $\hat{G}_{12}(z)$ and $\hat{G}_{21}(z)$ correspond to their $\sqrt{2} \tilde{G}_{+}(z)$ and $\sqrt{2} \tilde{G}_{-}(z)$ respectively because their $\delta_{a b}$ is normalized by $\frac{1}{2}$ (not by 1 ).

${ }^{39}$ If one takes the notations for the spin- $\frac{3}{2}$ currents in [1] using $(++,+-,-+,--)$, then one can express (3.19) more concisely rather than column notation.
} 


\begin{tabular}{|c|c|}
\hline U(1) charge & 11 currents of large $\mathcal{N}=4$ nonlinear superconformal algebra \\
\hline$\frac{2 k}{(5+k)}$ & $\hat{B}_{-}\left(\equiv \hat{B}_{1}-i \hat{B}_{2}\right)$ \\
\hline$\frac{(3+k)}{(5+k)}$ & $\hat{G}_{21}$ \\
\hline$\frac{(-3+k)}{(5+k)}$ & $\hat{G}_{11}$ \\
\hline$\frac{6}{(5+k)}$ & $\hat{A}_{-}\left(\equiv \hat{A}_{1}-i \hat{A}_{2}\right)$ \\
\hline 0 & $\hat{A}_{3}, \quad \hat{B}_{3}, \quad \hat{T}$ \\
\hline$-\frac{6}{(5+k)}$ & $\hat{A}_{+}\left(\equiv \hat{A}_{1}+i \hat{A}_{2}\right)$ \\
\hline$-\frac{(-3+k)}{(5+k)}$ & $\hat{G}_{22}$ \\
\hline$-\frac{(3+k)}{(5+k)}$ & $\hat{G}_{12}$ \\
\hline$-\frac{2 k}{(5+k)}$ & $\hat{B}_{+}\left(\equiv \hat{B}_{1}+i \hat{B}_{2}\right)$ \\
\hline
\end{tabular}

Table 1. The U(1) charges for the 11 currents from (3.19). The U(1) charge conservation can be seen from the equations in appendix (A.3), (A.5), (A.7) and (A.9). The first four currents with positive $(k>3) \mathrm{U}(1)$ charges have their conjugated currents with each opposite (negative) $\mathrm{U}(1)$ charge.

However, the $\mathrm{U}(1)$ charge assignment will choose the right candidate for the above spin- $\frac{7}{2}$ field by removing the unwanted terms which do not have the correct $\mathrm{U}(1)$ charge. ${ }^{40}$

Therefore, the large $\mathcal{N}=4$ nonlinear algebra is generated by (3.2), (3.5), (3.6), (3.10) and (3.13) and some of the algebra are given in (3.7), (3.11) and (3.18). The complete algebra is summarized in appendix A. ${ }^{41}$

\section{Higher spin currents in the Wolf space coset minimal model}

According to the observation of [1], the 16 lowest extra currents, appearing in the equation (2.33) of [1], consist of single spin-1, four spin- $\frac{3}{2}$, six spin-2, four spin- $\frac{5}{2}$, and single spin3 currents. Rearranging them in terms of $\mathcal{N}=2$ multiplets [47], one has four $\mathcal{N}=2$

\footnotetext{
${ }^{40}$ One finds the $\mathrm{U}(1)$ charges for the WZW affine currents. The fields having $\mathrm{U}(1)$ charge $\frac{k}{(5+k)}$ are given by $K^{m}(z)$ and $J^{a}(z)$ where $m=1, \cdots, 3$ and $a=10, \cdots, 12$. The fields with $\mathrm{U}(1)$ charge $-\frac{k}{(5+k)}$ are their conjugated ones $K^{\bar{m}}(z)$ and $J^{\bar{a}}(z)$ where $\bar{m}=\overline{1}, \cdots, \overline{3}$ and $\bar{a}=\overline{10}, \cdots, \overline{12}$. The fields with vanishing $\mathrm{U}(1)$ charge are given by $K^{m}(z), \bar{D} K^{m}(z)$ and $J^{9}(z)$ where $m=4, \cdots, 8$ (and their conjugated ones $K^{\bar{m}}(z)$, $D K^{\bar{m}}(z)$ and $J^{\overline{9}}(z)$ where $\left.\bar{m}=\overline{4}, \cdots, \overline{8}\right)$. The $\mathrm{U}(1)$ charges of the remaining WZW affine currents are undecided.

${ }^{41}$ In particular, when $\hat{k}^{+}=\hat{k}^{-}$(or $k=3$ ), the $\mathcal{N}=4$ nonlinear superconformal algebra becomes the $\mathrm{SO}(\mathcal{N}=4)$ Knizhnik-Bershadsky algebra $[43,44]$ with central charges $c=9$ and $c_{\text {Wolf }}=\frac{27}{4}$ along the line of $[15,24]$. Therefore, the higher spin currents in next section will lead to an extension of SO(4) KnizhnikBershadsky algebra at $c=9$. Furthermore, according to $[7,45,46]$, the $\mathcal{N}=3$ linear superconformal algebra can be reduced to the $\mathrm{SO}(\mathcal{N}=3)$ Knizhnik-Bershadsky (nonlinear) algebra by decoupling the fermion of spin- $\frac{1}{2}$. The central charge is reduced with $\frac{1}{2}$. The exact field redefinitions in order to see this in the present context should be done. See also [38] in the $\mathcal{N}=2$ superspace approach.
} 
supercurrents

$$
\begin{aligned}
& \left(1, \frac{3}{2}, \frac{3}{2}, 2\right):\left(T^{(1)}, T_{+}^{\left(\frac{3}{2}\right)}, T_{-}^{\left(\frac{3}{2}\right)}, T^{(2)}\right), \\
& \left(\frac{3}{2}, 2,2, \frac{5}{2}\right):\left(U^{\left(\frac{3}{2}\right)}, U_{+}^{(2)}, U_{-}^{(2)}, U^{\left(\frac{5}{2}\right)}\right), \\
& \left(\frac{3}{2}, 2,2, \frac{5}{2}\right):\left(V^{\left(\frac{3}{2}\right)}, V_{+}^{(2)}, V_{-}^{(2)}, V^{\left(\frac{5}{2}\right)}\right), \\
& \left(2, \frac{5}{2}, \frac{5}{2}, 3\right):\left(W^{(2)}, W_{+}^{\left(\frac{5}{2}\right)}, W_{-}^{\left(\frac{5}{2}\right)}, W^{(3)}\right) .
\end{aligned}
$$

This section will consider the particular supersymmetric Wolf space coset minimal model (2.1) and the higher spin currents in (4.1) will be determined. ${ }^{42}$

\subsection{Construction of higher spin currents of spins $\left(1, \frac{3}{2}, \frac{3}{2}, 2\right)$}

How does one determine the spin-1 current in (4.1)? The basic $24 \mathcal{N}=2$ WZW affine currents of spins $\frac{1}{2}$ are given in terms of $K^{m}(z), K^{\bar{m}}(z), J^{a}(z)$ and $J^{\bar{a}}(z)$ in (2.9). Then the general spin- 1 current can be obtained from these by considering the quadratic expressions. Furthermore, the basic spin- $1 \mathcal{N}=2$ WZW affine currents are given by $\bar{D} K^{m}(z), D K^{\bar{m}}(z), \bar{D} J^{a}(z)$ and $D J^{\bar{a}}(z)$. The linear combinations of these currents should be added into the most general spin-1 current $T^{(1)}(z)$ we are looking for. Then one can write down, with 300 coefficient functions which depend on the level $k$, as

$$
\begin{aligned}
T^{(1)}(z)= & \sum_{m, n=1}^{8} c_{m, n} K^{m} K^{n}(z)+\sum_{m=1}^{8} \sum_{\bar{n}=\overline{1}}^{\overline{8}} c_{m, \bar{n}} K^{m} K^{\bar{n}}(z)+\sum_{m=1}^{8} \sum_{a=9}^{12} c_{m, a} K^{m} J^{a}(z) \\
& +\sum_{m=1}^{8} \sum_{\bar{a}=\overline{9}}^{\overline{12}} c_{m, \bar{a}} K^{m} J^{\bar{a}}(z)+\sum_{\bar{m}, \bar{n}=\overline{1}}^{\overline{8}} c_{\bar{m}, \bar{n}} K^{\bar{m}} K^{\bar{n}}(z)+\sum_{\bar{m}=\overline{1}}^{12} \sum_{a=9}^{12} c_{\bar{m}, a} K^{\bar{m}} J^{a}(z) \\
& +\sum_{\bar{m}=\overline{1}}^{\overline{8}} \sum_{\bar{a}=\overline{9}}^{\overline{12}} c_{\bar{m}, \bar{a}} K^{\bar{m}} J^{\bar{a}}(z)+\sum_{a, b=9}^{12} c_{a, b} J^{a} J^{b}(z)+\sum_{a=9}^{12} \sum_{\bar{b}=\overline{9}}^{\overline{12}} c_{a, \bar{b}} J^{a} J^{\bar{b}}(z)+\sum_{\bar{a}=\overline{9}}^{\overline{12}} c_{\bar{a}} D J^{\bar{a}}(z) \\
& +\sum_{\bar{a}, \bar{b}=\overline{9}}^{1 \overline{2}} c_{\bar{a}, \bar{b}} J^{\bar{a}} J^{\bar{b}}(z)+\sum_{m=1}^{8} c_{m} \bar{D} K^{m}(z)+\sum_{\bar{m}=\overline{1}} c_{\bar{m}} D K^{\bar{m}}(z)+\sum_{a=9}^{12} c_{a} \bar{D} J^{a}(z) .
\end{aligned}
$$

We would like to determine the coefficient functions appearing in (4.2) explicitly. Since the regularity conditions between the spin- 1 current $\mathrm{U}(z)$ and the spin- $\frac{1}{2}$ currents, $F_{11}(z)$,

\footnotetext{
${ }^{42}$ Let us emphasize that in the Wolf space coset model (2.1) written in terms of $\mathcal{N}=2$ superspace (effectively $\mathcal{N}=1$ superspace due to the constraints), the higher spin currents should commute with the $\mathcal{N}=2$ WZW affine currents (of spin- $\frac{1}{2}$ fields and its superpartner spin-1 fields) living on the denominator $\mathrm{SU}(3)$ (the explicit $8+8$ fields will be described in the footnote 53), commute with those of three spin- $\frac{1}{2}$ currents living on the denominator $\mathrm{SU}(2)$ (the fields $F_{11}(z), F_{22}(z)$ and $\left(F_{21}+F_{12}\right)(z)$ ), and commute with the spin-1 and its superpartner spin- $\frac{1}{2}$ currents living on the denominator $\mathrm{U}(1)$ (i.e. the field $\mathrm{U}(z)$ with $(2.38)$ and the field $\left.\left(F_{21}-F_{12}\right)(z)\right)$. Once again, the OPEs between the higher spin currents and the three $\mathrm{SU}(2)$ currents, $\left[\bar{D} F_{11}(z), D F_{22}(z)\right.$ and $\left.\left(\bar{D} F_{21}+D F_{12}\right)(z)\right]$, which are the superpartner of above three spin- $\frac{1}{2}$ currents DO have singular terms. Therefore, the $11+16=27$ (higher spin) currents commute with $16+3+2=21$ Wolf space denominator currents among 48 currents in the group $\mathrm{SU}(5)$.
} 
$F_{12}(z), F_{21}(z)$ and $F_{22}(z)$ that live in section 2 and the spin- 1 current $T^{(1)}(w)$ are preserved in this extended nonlinear algebra, the following relations, together with (2.36), (2.26), (2.23), (2.20), (2.28) and (4.2), should satisfy

$$
\mathrm{U}(z) T^{(1)}(w)=+\cdots, \quad F_{a}(z) T^{(1)}(w)=+\cdots, \quad a=11,12,21,22 .
$$

Then the remaining undetermined coefficient functions can be fixed by the following primary field condition under the stress tensor (3.2)

$$
\hat{T}(z) T^{(1)}(w)=\frac{1}{(z-w)^{2}} T^{(1)}(w)+\frac{1}{(z-w)} \partial T^{(1)}(w)+\cdots .
$$

This OPE (4.4) implies the following result

$$
\left.T^{(1)}(z) \hat{T}(w)\right|_{\frac{1}{(z-w)}}=0 .
$$

In other words, the commutator $\left[T_{0}^{(1)}, \hat{T}\right]$ between the zero mode of $T^{(1)}$ and $\hat{T}$ vanishes. Then we are left with seven unknown coefficient functions.

All the first-order singular terms between the above spin- 1 current and other six spin-1 currents can be obtained from the defining equations (3.9), (3.12) and (4.2) and by requiring that the commutators between the zero mode $T_{0}^{(1)}$ and six spin-1 currents should vanish [1]

$$
\left.T^{(1)}(z) \hat{A}_{i}(w)\right|_{\frac{1}{(z-w)}}=0,\left.\quad T^{(1)}(z) \hat{B}_{i}(w)\right|_{\frac{1}{(z-w)}}=0
$$

all the remaining coefficient functions are completely determined except an overall constant.

It turns out that the lowest higher spin-1 current (4.2) can be obtained as follows: ${ }^{43}$

$$
\begin{aligned}
T^{(1)}(z)= & -\frac{5(3 i+\sqrt{3})}{6(5+k)} \bar{D} K^{7}(z)+\frac{(-5 i \sqrt{3}+9 \sqrt{5})}{6 \sqrt{2}(5+k)} \bar{D} K^{8}(z)-\frac{5(-3 i+\sqrt{3})}{6(5+k)} D K^{\overline{7}}(z) \\
& +\frac{(5 i \sqrt{3}+9 \sqrt{5})}{6 \sqrt{2}(5+k)} D K^{\overline{8}}(z)-\frac{1}{(5+k)}\left(\sum_{(a, \bar{a})=(10, \overline{10})}^{(12, \overline{1} 2)} J^{a} J^{\bar{a}}-\sum_{(m, \bar{m})=(1, \overline{1})}^{(3, \overline{3})} K^{m} K^{\bar{m}}\right)(z) .
\end{aligned}
$$

The field contents of (4.7) look similar to those of $\hat{A}_{3}(z)$ in (3.9) except that the former does not have the term of $J^{9} J^{\overline{9}}(z)$. One can easily check that this spin-1 current is new primary current in the sense that this cannot be written in terms of given six spin- 1 currents: $\hat{A}_{i}$ and $\hat{B}_{i}$. Furthermore, the OPE between the spin-1 currents (4.7) does not have a first-order pole: ${ }^{44}$

$$
\left.T^{(1)}(z) T^{(1)}(w)\right|_{\frac{1}{(z-w)}}=0
$$

${ }^{43}$ The first four terms of $(4.7)$ can be written as $-\frac{2 i \sqrt{\frac{5}{3}}}{(5+k)} \mathrm{U}(z)+\left(-\frac{(15 i+5 \sqrt{3}-i \sqrt{5}+\sqrt{15})}{6(5+k)} \bar{D} K^{7}+\frac{2 \sqrt{10}}{3(5+k)} \bar{D} K^{8}\right)+$ $\left(-\frac{(-15 i+5 \sqrt{3}+i \sqrt{5}+\sqrt{15})}{6(5+k)} D K^{\overline{7}}+\frac{2 \sqrt{10}}{3(5+k)} D K^{\overline{8}}\right)$ where the last two fields correspond to the Wolf space denominator $\mathrm{SU}(3)$ current. This spin-1 current has both subgroup indices and coset indices of Wolf space.

${ }^{44}$ One obtains the $\operatorname{OPE} T^{(1)}(z) T^{(1)}(w)=\frac{1}{(z-w)^{2}}\left[\frac{6 k}{(5+k)}\right]+\cdots$. 
Therefore, this spin-1 current will play an important role in the construction of higher spin currents because this will generate, in principle, all the higher spin currents with the help of the currents in the $\mathcal{N}=4$ nonlinear algebra described in previous section.

Consider the next OPE between the spin- $\frac{3}{2}$ current (3.17) and the spin-1 current (4.7) to determine the other current in the higher spin currents of spins $\left(1, \frac{3}{2}, \frac{3}{2}, 2\right)$. Usually, the $\mathcal{N}=2$ description for this particular OPE produces the second component of $\mathcal{N}=2$ superprimary current. The result can be expressed as

$$
\hat{G}_{21}(z) T^{(1)}(w)=\frac{1}{(z-w)}\left[\hat{G}_{21}+2 T_{+}^{\left(\frac{3}{2}\right)}\right](w)+\cdots,
$$

where the new spin- $\frac{3}{2}$ current (in the sense that this cannot be written in terms of combination of known currents) can be obtained

$$
T_{+}^{\left(\frac{3}{2}\right)}(w)=-\frac{\sqrt{2}}{(5+k)} \sum_{(a, \bar{a})=(10, \overline{10})}^{(12, \overline{12})} J^{a} D J^{\bar{a}}(w)+\text { other cubic terms. }
$$

The field contents of this current can be seen from the current $\hat{G}_{21}(z)(3.17)$ where there are quadratic and cubic terms with the two indices among $1,2,3, \overline{1}, \overline{2}$ and $\overline{3}$. From the right hand side of (4.9), the $\mathrm{U}(1)$ charges of two currents appearing in the first-order pole are the same.

There are no singular terms in the OPE between this spin- $\frac{3}{2}$ current (4.10) and the spin-1 current (2.36). The OPEs between the spin- $\frac{3}{2}$ current (4.10) and the spin- $\frac{1}{2}$ currents (2.26), (2.23), (2.20), and (2.28) (also the spin-1 current (2.36)) do not contain any singular terms as in (4.3):

$$
\mathrm{U}(z) T_{+}^{\left(\frac{3}{2}\right)}(w)=+\cdots, \quad F_{a}(z) T_{+}^{\left(\frac{3}{2}\right)}(w)=+\cdots, \quad a=11,12,21,22 .
$$

Note that the normalization of spin-1 current $T^{(1)}(z)$ in (4.7) (i.e. the footnote 44 ) was fixed by the following $\mathrm{OPE}^{45}$

$$
T^{(1)}(z) T_{+}^{\left(\frac{3}{2}\right)}(w)=\frac{1}{(z-w)} T_{+}^{\left(\frac{3}{2}\right)}(w)+\cdots
$$

That is, the right hand side of (4.12) can be described as $(5+k) A(N, k) T_{+}^{\left(\frac{3}{2}\right)}(w)$ with normalization $A(N, k)$. By taking the $\mathrm{U}(1)$ charge under the $T^{(1)}$ spin-1 current to be 1 , the normalization constant is given by $A(N, k)=\frac{1}{(5+k)}$ which appears in (4.7). The OPE (4.12) implies that the $\mathrm{U}(1)$ charge of $T^{(1)}(z)$ is equal to zero.

The next OPE between the spin- $\frac{3}{2}$ current (3.16) and the spin-1 current (4.7) can be calculated to obtain the other remaining spin- $\frac{3}{2}$ current in the higher spin currents of spins $\left(1, \frac{3}{2}, \frac{3}{2}, 2\right)$. As observed before, the reason why one considers this particular OPE is the

\footnotetext{
${ }^{45}$ We will not consider the OPEs between the higher spin currents themselves in this paper. Of course, they should be calculated to complete the full structure of the extended large $\mathcal{N}=4$ nonlinear algebra and will appear near future [48].
} 
fact that the $\mathcal{N}=2$ description of this OPE provides the third component of $\mathcal{N}=2$ superprimary current. The result can be expressed as follows:

$$
\hat{G}_{12}(z) T^{(1)}(w)=\frac{1}{(z-w)}\left[-\hat{G}_{12}+2 T_{-}^{\left(\frac{3}{2}\right)}\right](w)+\cdots,
$$

where the new spin- $\frac{3}{2}$ current can be written as

$$
T_{-}^{\left(\frac{3}{2}\right)}(w)=-\frac{\sqrt{2}}{(5+k)} \sum_{(a, \bar{a})=(10, \overline{10})}^{(12, \overline{2})} \bar{D} J^{a} J^{\bar{a}}(w)+\text { other cubic and linear terms. }
$$

The field contents of this current can be seen from the current $\hat{G}_{12}(z)(3.16)$ where there are quadratic and cubic terms with the two indices among $1,2,3, \overline{1}, \overline{2}$ and $\overline{3}$. The U(1) charge of two currents in the first-order pole of (4.13) are the same. The OPE (4.13) is conjugated to the OPE (4.9).

No singular terms in the OPE between this spin- $\frac{3}{2}$ current (4.14) and the spin-1 current (2.36) exist. The OPEs between the spin- $\frac{3}{2}$ current (4.14) and the spin- $\frac{1}{2}$ currents (2.26), (2.23), (2.20), and (2.28) do not contain any singular terms as in (4.3) and (4.11):

$$
\mathrm{U}(z) T_{-}^{\left(\frac{3}{2}\right)}(w)=+\cdots, \quad F_{a}(z) T_{-}^{\left(\frac{3}{2}\right)}(w)=+\cdots, \quad a=11,12,21,22 .
$$

Consider the spin- $\frac{3}{2}$ current (3.17) and the spin- $\frac{3}{2}$ current (4.14) to determine the last component spin-2 current. The result can be expressed as

$$
\begin{aligned}
\hat{G}_{21}(z) T_{-}^{\left(\frac{3}{2}\right)}(w)= & \frac{1}{(z-w)^{3}} \frac{6 k}{(5+k)}+\frac{1}{(z-w)^{2}}\left[T^{(1)}+\frac{2 i}{(5+k)}\left(-3 \hat{A}_{3}-k \hat{B}_{3}\right)\right](w) \\
& +\frac{1}{(z-w)}\left[\frac{6 k}{(3+7 k)} \hat{T}+T^{(2)}+\frac{1}{2} \partial\left(T^{(1)}+\frac{2 i}{(5+k)}\left(-3 \hat{A}_{3}-k \hat{B}_{3}\right)\right)\right](w) \\
& +\cdots,
\end{aligned}
$$

where the last component of the higher spin current of spins $\left(1, \frac{3}{2}, \frac{3}{2}, 2\right)$ can be described as

$$
\begin{aligned}
T^{(2)}(w)= & -\frac{2}{(5+k)^{3}} \sum_{(a, \bar{a})=(10, \overline{10})}^{(12, \overline{1})} J^{9} J^{a} J^{\overline{9}} J^{\bar{a}}(w) \\
& + \text { other quartic, cubic, quadratic and linear terms. }
\end{aligned}
$$

The complete expression is not presented here. In the first-order pole of (4.16), the new spin-2 current arises and another primary spin-2 current (3.2). The relative coefficient $\frac{1}{2}$ in the descendant field of the spin- 1 current in the second-order pole can be obtained from the formula in the OPE of two (quasi) primary fields. Of course, this spin-2 current cannot be written in terms of other known currents. The $\mathrm{U}(1)$ charges for the currents in the first-order pole of (4.16) should be the same.

No singular terms in the following OPEs exist as in (4.3), (4.11) and (4.15):

$$
\mathrm{U}(z) T^{(2)}(w)=+\cdots, \quad F_{a}(z) T^{(2)}(w)=+\cdots, \quad a=11,12,21,22 .
$$


As in (4.5), (4.6) and (4.8), the commutator between the zero mode $T_{0}^{(1)}$ and the spin-2 current $T^{(2)}$ vanishes: ${ }^{46}$

$$
\left.T^{(1)}(z) T^{(2)}(w)\right|_{\frac{1}{(z-w)}}=0 .
$$

Therefore, the higher spin currents of spins $\left(1, \frac{3}{2}, \frac{3}{2}, 2\right)$ are determined completely: (4.7), (4.10), (4.14) and (4.17). Some of the OPEs between these higher spin currents and the currents from the large $\mathcal{N}=4$ nonlinear superconformal algebra are presented in (4.9), (4.13) and (4.16). The remaining OPEs are given in appendix B.1 and appendix C.1.

\subsection{Construction of higher spin currents of spins $\left(\frac{3}{2}, 2,2, \frac{5}{2}\right)$}

What happens when the other spin- $\frac{3}{2}$ current $\hat{G}_{11}(z)$ or $\hat{G}_{22}(z)$ rather than $\hat{G}_{21}(z)$ or $\hat{G}_{12}(z)$ acts on the lowest component of previous multiplet $\left(1, \frac{3}{2}, \frac{3}{2}, 2\right)$ ? Let us focus on the OPE $\hat{G}_{11}(z) T^{(1)}(w)$. From the explicit expressions (3.14) and (4.7), the following OPE can be calculated easily

$$
\hat{G}_{11}(z) T^{(1)}(w)=\frac{1}{(z-w)}\left[\hat{G}_{11}+2 U^{\left(\frac{3}{2}\right)}\right](w)+\cdots
$$

where the new spin- $\frac{3}{2}$ current which cannot be written in terms of other currents can be expressed as

$$
\begin{aligned}
U^{\left(\frac{3}{2}\right)}(w)= & -\frac{i \sqrt{2}}{(5+k)^{2}} \sum_{(a, \bar{a})=(10, \overline{10})}^{(12, \overline{1})} J^{9} J^{a} J^{\bar{a}}(w) \\
& + \text { other cubic, quadratic and linear terms. }
\end{aligned}
$$

Some of the fields in (4.21) can be seen from the $\hat{G}_{11}(w)$ but the following three terms $K^{2} K^{\overline{4}} J^{10}(w), K^{3} K^{\overline{5}} J^{10}(w)$ and $K^{3} K^{\overline{6}} J^{11}(w)$ occur in (4.21) newly. The U(1) charges in the first-order pole of (4.20) should be the same. As stated before, the following regularity conditions, as in (4.18), satisfy

$$
\mathrm{U}(z) U^{\left(\frac{3}{2}\right)}(w)=+\cdots, \quad F_{a}(z) U^{\left(\frac{3}{2}\right)}(w)=+\cdots, \quad a=11,12,21,22 .
$$

Consider the next OPE between the spin- $\frac{3}{2}$ current (3.14) and the spin- $\frac{3}{2}$ current (4.10). The result is as follows:

$$
\begin{aligned}
\hat{G}_{11}(z) T_{+}^{\left(\frac{3}{2}\right)}(w)= & \frac{1}{(z-w)^{2}} \frac{2 k}{(5+k)}\left[i \hat{B}_{-}\right](w) \\
& +\frac{1}{(z-w)}\left[-U_{+}^{(2)}-\frac{1}{(5+k)} 4 \hat{A}_{3} \hat{B}_{-}+\frac{1}{2} \frac{2 k}{(5+k)} i \partial \hat{B}_{-}\right](w) \\
& +\cdots,
\end{aligned}
$$

\footnotetext{
${ }^{46}$ More explicitly, one has the OPE $T^{(1)}(z) T^{(2)}(w)=\frac{1}{(z-w)^{2}}\left[-\frac{6}{(5+k)} i \hat{A}_{3}-\frac{2 k}{(5+k)} i \hat{B}_{3}+\frac{(3+k)}{(3+7 k)} T^{(1)}\right](w)+\cdots$
} 
where the new spin- 2 current is given by ${ }^{47}$

$$
\begin{aligned}
U_{+}^{(2)}(w)= & \frac{2 i}{(5+k)^{3}}\left(-K^{1} J^{9} J^{10} J^{\overline{9}}+K^{1} J^{10} J^{11} J^{\overline{11}}+K^{1} J^{10} J^{12} J^{12}\right)(w) \\
& + \text { other quartic, cubic and quadratic terms. }
\end{aligned}
$$

Note that the second term $\hat{A}_{3}\left(\hat{B}_{1}-i \hat{B}_{2}\right)$ in the first order pole of (4.23) is primary field under the stress tensor (3.2) because the current $\hat{A}_{3}(z)$ and the current $\left(\hat{B}_{1}-i \hat{B}_{2}\right)(z)$ are primary and commute with each other. Furthermore, each term of the spin-2 current (4.24) contains $K^{1}(z), K^{2}(z)$ or $K^{3}(z)$. There are no composite terms consisting of $J^{a}(z), J^{\bar{a}}(z)$, $\bar{D} J^{a}(z)$ or $D J^{\bar{a}}(z)$.

As in (4.22), the following OPEs satisfy the regular conditions

$$
\mathrm{U}(z) U_{+}^{(2)}(w)=+\cdots, \quad F_{a}(z) U_{+}^{(2)}(w)=+\cdots, \quad a=11,12,21,22 .
$$

Furthermore, from the equations (4.7) and (4.24), one checks, as in (4.19), the following vanishing first-order pole

$$
\left.T^{(1)}(z) U_{+}^{(2)}(w)\right|_{\frac{1}{(z-w)}}=0 .
$$

Now let us consider the spin- $\frac{3}{2}$ current (3.14) acting on the spin- $\frac{3}{2}$ current (4.14)

$$
\begin{aligned}
\hat{G}_{11}(z) T_{-}^{\left(\frac{3}{2}\right)}(w)= & \frac{1}{(z-w)^{2}} \frac{6}{(5+k)}\left[i \hat{A}_{+}\right](w) \\
& +\frac{1}{(z-w)}\left[-U_{-}^{(2)}+\frac{1}{2} \frac{6}{(5+k)} i \partial \hat{A}_{+}\right](w)+\cdots,
\end{aligned}
$$

where the other spin- 2 current which obtained by subtracting the descendant fields can be described $a^{48}$

$$
\begin{aligned}
U_{-}^{(2)}(w)= & \frac{2 i}{(5+k)^{2}} \sum_{(a, \bar{a})=(10, \overline{10})}^{(12, \overline{12})} J^{9} \bar{D} J^{a} J^{\bar{a}}(w) \\
& + \text { other quartic, cubic, quadratic and linear terms. }
\end{aligned}
$$

Compared to the previous spin-2 current (4.24), the first six terms (and the last term) of (4.28) contain only $J^{a}(z), J^{\bar{a}}(z), \bar{D} J^{a}(z)$ or $D J^{\bar{a}}(z)$. One realizes that the $\mathrm{U}(1)$ charge of $U_{-}^{(2)}(z)$ is the same as the one of $\left(\hat{A}_{1}+i \hat{A}_{2}\right)(z)$ from the OPE (4.27).

\footnotetext{
${ }^{47}$ From the OPE

$$
\hat{G}_{21}(z) U^{\left(\frac{3}{2}\right)}(w)=\frac{1}{(z-w)^{2}} \frac{2 k}{(5+k)}\left[-i \hat{B}_{-}\right](w)+\frac{1}{(z-w)}\left[U_{+}^{(2)}-\frac{1}{2} \frac{2 k}{(5+k)} i \partial \hat{B}_{-}\right](w)+\cdots,
$$
}

it is natural to consider the extra spin-2 current in the first-order pole (i.e. the first-order pole subtracted by the descendant terms) as a second component of higher spin currents of spins $\left(\frac{3}{2}, 2,2, \frac{5}{2}\right)$.

${ }^{48}$ In this case, one has also

$$
\hat{G}_{12}(z) U^{\left(\frac{3}{2}\right)}(w)=\frac{1}{(z-w)^{2}} \frac{6}{(5+k)}\left[i \hat{A}_{+}\right](w)+\frac{1}{(z-w)}\left[U_{-}^{(2)}+\frac{1}{2} \frac{6}{(5+k)} i \partial \hat{A}_{+}\right](w)+\cdots .
$$

After subtracting the descendant term in the first-order pole, the extra spin-2 current with plus sign is the third component of the higher spin currents of spins $\left(\frac{3}{2}, 2,2, \frac{5}{2}\right)$. 
As one expects in (4.25), the following OPEs are satisfied

$$
\mathrm{U}(z) U_{-}^{(2)}(w)=+\cdots, \quad F_{a}(z) U_{-}^{(2)}(w)=+\cdots, \quad a=11,12,21,22 .
$$

One also checks that the first-order pole of the following OPE vanishes as in (4.26)

$$
\left.T^{(1)}(z) U_{-}^{(2)}(w)\right|_{\frac{1}{(z-w)}}=0
$$

As in (4.16), the last component spin- $\frac{5}{2}$ current can be calculated from the following OPE

$$
\begin{aligned}
\hat{G}_{21}(z) U_{-}^{(2)}(w)= & \frac{1}{(z-w)^{2}}\left[\frac{(3+2 k)}{(5+k)} \hat{G}_{11}+\frac{4(2+k)}{(5+k)} U^{\left(\frac{3}{2}\right)}\right](w) \\
& +\frac{1}{(z-w)}\left[U^{\left(\frac{5}{2}\right)}+\frac{1}{3} \partial\left(\frac{(3+2 k)}{(5+k)} \hat{G}_{11}+\frac{4(2+k)}{(5+k)} U^{\left(\frac{3}{2}\right)}\right)\right](w)+\cdots,
\end{aligned}
$$

where the new spin- $\frac{5}{2}$ current can be described as

$$
\begin{aligned}
U^{\left(\frac{5}{2}\right)}(w)= & \frac{4 i \sqrt{2}}{(5+k)^{4}}\left(J^{9} J^{10} J^{11} J^{\overline{10}} J^{\overline{11}}+J^{9} J^{10} J^{12} J^{\overline{10}} J^{\overline{12}}+J^{9} J^{11} J^{12} J^{\overline{11}} J^{\overline{12}}\right)(w) \\
& + \text { other quintic, quartic, cubic, quadratic and linear terms. }
\end{aligned}
$$

Again, the U(1) charge conservation leads to the fact that the three currents in (4.31) in the first-order pole have the same U(1) charge. The complete expression for (4.32) is not presented here. Moreover, the following regularity conditions which are similar to (4.29), satisfy

$$
\mathrm{U}(z) U^{\left(\frac{5}{2}\right)}(w)=+\cdots, \quad F_{a}(z) U^{\left(\frac{5}{2}\right)}(w)=+\cdots, \quad a=11,12,21,22 .
$$

Therefore, the higher spin currents of spins $\left(\frac{3}{2}, 2,2, \frac{5}{2}\right)$ are determined completely: (4.21), (4.24), (4.28) and (4.32). Some of the OPEs between these higher spin currents and the currents from the large $\mathcal{N}=4$ nonlinear superconformal algebra are presented in (4.20), (4.23), (4.27) and (4.31). The remaining OPEs are given in appendix B.2 and appendix C.2.

\subsection{Construction of higher spin currents of spins $\left(\frac{3}{2}, 2,2, \frac{5}{2}\right)$}

In this subsection, we consider the second case when the other spin- $\frac{3}{2}$ current $\hat{G}_{22}(z)$ acts on the lowest component of previous multiplet $\left(1, \frac{3}{2}, \frac{3}{2}, 2\right)$ :

$$
\hat{G}_{22}(z) T^{(1)}(w)=\frac{1}{(z-w)}\left[-\hat{G}_{22}+2 V^{\left(\frac{3}{2}\right)}\right](w)+\cdots,
$$

where the lowest component spin- $\frac{3}{2}$ current can be expressed as

$$
\begin{aligned}
V^{\left(\frac{3}{2}\right)}(w)= & -\frac{i \sqrt{2}}{(5+k)^{2}} \sum_{(a, \bar{a})=(10, \overline{10})}^{(12, \overline{1})} J^{a} J^{\overline{9}} J^{\bar{a}}(w) \\
& + \text { other cubic, quadratic and linear terms. }
\end{aligned}
$$


The field contents of (4.35) look similar to those of (4.21). The barred and unbarred indices in (4.21) are replaced by unbarred and barred ones in (4.35) respectively. The $\mathrm{U}(1)$ charges of the two currents in (4.34) are the same. The OPE (4.34) is conjugated to the OPE (4.20). As in previous results in (4.33), the spin- $\frac{3}{2}$ current satisfies

$$
\mathrm{U}(z) V^{\left(\frac{3}{2}\right)}(w)=+\cdots, \quad F_{a}(z) V^{\left(\frac{3}{2}\right)}(w)=+\cdots, \quad a=11,12,21,22 .
$$

Consider the next OPE

$$
\begin{aligned}
\hat{G}_{22}(z) T_{+}^{\left(\frac{3}{2}\right)}(w)= & \frac{1}{(z-w)^{2}} \frac{6}{(5+k)}\left[i \hat{A}_{-}\right](w) \\
& +\frac{1}{(z-w)}\left[-V_{+}^{(2)}+\frac{1}{2} \frac{6}{(5+k)} i \partial \hat{A}_{-}\right](w)+\cdots
\end{aligned}
$$

where the new spin-2 current occurs in the first order pole of (4.37) and the result is as follows: ${ }^{49}$

$$
\begin{aligned}
V_{+}^{(2)}(w)= & \frac{2 i}{(5+k)^{2}} \sum_{(a, \bar{a})=(10, \overline{10})}^{(12, \overline{12})} J^{a} J^{\overline{9}} D J^{\bar{a}}(w) \\
& + \text { other quartic, cubic, quadratic and linear terms. }
\end{aligned}
$$

The field contents of (4.38) can be obtained after the barred and unbarred indices in (4.28) are replaced by unbarred and barred ones respectively. The OPE (4.37) looks very similar to (4.27): they are conjugated to each other under the $\mathrm{U}(1)$ charge.

One can see immediately that the following regularity conditions hold, as in (4.33) and (4.36),

$$
\mathrm{U}(z) V_{+}^{(2)}(w)=+\cdots, \quad F_{a}(z) V_{+}^{(2)}(w)=+\cdots, \quad a=11,12,21,22 .
$$

Furthermore, the first-order pole in the $\operatorname{OPE} T^{(1)}(z) V_{+}^{(2)}(w)$ vanishes, as in (4.30),

$$
\left.T^{(1)}(z) V_{+}^{(2)}(w)\right|_{\frac{1}{(z-w)}}=0 .
$$

One describes the following OPE

$$
\begin{aligned}
\hat{G}_{22}(z) T_{-}^{\left(\frac{3}{2}\right)}(w)= & \frac{1}{(z-w)^{2}} \frac{2 k}{(5+k)}\left[i \hat{B}_{+}\right](w) \\
& +\frac{1}{(z-w)}\left[-V_{-}^{(2)}+\frac{1}{2} \frac{2 k}{(5+k)} i \partial \hat{B}_{+}+\frac{1}{(5+k)} 4 \hat{A}_{3} \hat{B}_{+}\right](w) \\
& +\cdots
\end{aligned}
$$

\footnotetext{
${ }^{49}$ One obtains

$$
\hat{G}_{21}(z) V^{\left(\frac{3}{2}\right)}(w)=\frac{1}{(z-w)^{2}} \frac{6}{(5+k)}\left[i \hat{A}_{-}\right](w)+\frac{1}{(z-w)}\left[V_{+}^{(2)}+\frac{1}{2} \frac{6}{(5+k)} i \partial \hat{A}_{-}\right](w)+\cdots
$$
}

As before, the spin- 2 current with plus sign appears in the first-order pole after subtracting the descendant term. 
where the other spin- 2 current can be described as ${ }^{50}$

$$
\begin{aligned}
V_{-}^{(2)}(w)= & \frac{2 i}{(5+k)^{3}}\left(-K^{\overline{1}} J^{9} J^{\overline{9}} J^{\overline{10}}+K^{\overline{1}} J^{11} J^{\overline{10}} J^{\overline{11}}+K^{\overline{1}} J^{12} J^{\overline{10}} J^{\overline{12}}\right)(w) \\
& + \text { other quartic, cubic and quadratic terms. }
\end{aligned}
$$

The OPE (4.41) is conjugated to the OPE (4.23) under the U(1) charge. As before in (4.39), one has the following OPEs

$$
\mathrm{U}(z) V_{-}^{(2)}(w)=+\cdots, \quad F_{a}(z) V_{-}^{(2)}(w)=+\cdots, \quad a=11,12,21,22,
$$

and along the line of (4.40) the following relation holds

$$
\left.T^{(1)}(z) V_{-}^{(2)}(w)\right|_{\frac{1}{(z-w)}}=0 .
$$

Let us calculate the last component spin- $\frac{5}{2}$ current, as in (4.31),

$$
\begin{aligned}
\hat{G}_{21}(z) V_{-}^{(2)}(w)= & \frac{1}{(z-w)^{2}}\left[-\frac{(6+k)}{(5+k)} \hat{G}_{22}+\frac{2(7+k)}{(5+k)} V^{\left(\frac{3}{2}\right)}\right](w) \\
& +\frac{1}{(z-w)}\left[V^{\left(\frac{5}{2}\right)}+\frac{1}{3} \partial\left(-\frac{(6+k)}{(5+k)} \hat{G}_{22}+\frac{2(7+k)}{(5+k)} V^{\left(\frac{3}{2}\right)}\right)\right](w)+\cdots,
\end{aligned}
$$

where the new spin- $\frac{5}{2}$ current after subtracting the descendant fields can be described as

$$
\begin{aligned}
V^{\left(\frac{5}{2}\right)}(w)= & -\frac{4 i \sqrt{2}}{(5+k)^{4}}\left(J^{10} J^{11} J^{\overline{9}} J^{\overline{10}} J^{\overline{11}}+J^{10} J^{12} J^{\overline{9}} J^{\overline{10}} J^{12}+J^{11} J^{12} J^{\overline{9}} J^{\overline{1} 1} J^{\overline{12}}\right)(w) \\
& + \text { other quintic, quartic, cubic, quadratic and linear terms. }
\end{aligned}
$$

Furthermore one has the regularity conditions, similar to (4.36), (4.39) and (4.43), as follows:

$$
\mathrm{U}(z) V^{\left(\frac{5}{2}\right)}(w)=+\cdots, \quad F_{a}(z) V^{\left(\frac{5}{2}\right)}(w)=+\cdots, \quad a=11,12,21,22 .
$$

Therefore, the higher spin currents of spins $\left(\frac{3}{2}, 2,2, \frac{5}{2}\right)$ are determined completely: (4.35), (4.38), (4.42) and (4.46). Some of the OPEs between these higher spin currents and the currents from the large $\mathcal{N}=4$ nonlinear superconformal algebra are presented in (4.34), (4.37), (4.41) and (4.45). The remaining OPEs are given in appendix B.2 and appendix C.2.

\footnotetext{
${ }^{50}$ One obtains the following OPE

$$
\hat{G}_{12}(z) V^{\left(\frac{3}{2}\right)}(w)=\frac{1}{(z-w)^{2}} \frac{2 k}{(5+k)}\left[-i \hat{B}_{+}\right](w)+\frac{1}{(z-w)}\left[V_{-}^{(2)}-\frac{1}{2} \frac{2 k}{(5+k)} i \partial \hat{B}_{+}\right](w)+\cdots,
$$
}

where the spin-2 current with plus sign appears in the first-order pole. 


\subsection{Construction of higher spin currents of spins $\left(2, \frac{5}{2}, \frac{5}{2}, 3\right)$}

In this subsection, the final $\mathcal{N}=2$ multiplet can be determined. One way to see the presence of new spin-2 current is to calculate the following OPE, together with (3.15) and (4.21),

$$
\begin{aligned}
\hat{G}_{22}(z) U^{\left(\frac{3}{2}\right)}(w)= & -\frac{1}{(z-w)^{3}} \frac{6 k}{(5+k)}+\frac{1}{(z-w)^{2}}\left[\frac{2 i}{(5+k)}\left(3 \hat{A}_{3}-k \hat{B}_{3}\right)+T^{(1)}\right](w) \\
& +\frac{1}{(z-w)}\left[-W^{(2)}+\frac{1}{2} \partial\left(\frac{2 i}{(5+k)}\left(3 \hat{A}_{3}-k \hat{B}_{3}\right)+T^{(1)}\right)\right](w) \\
& +\cdots,
\end{aligned}
$$

where the new spin-2 current can be written as

$$
\begin{aligned}
W^{(2)}(w)= & \frac{4}{(5+k)^{3}}\left(J^{9} J^{10} J^{\overline{9}} J^{\overline{10}}+J^{9} J^{11} J^{\overline{9}} J^{\overline{11}}+J^{9} J^{12} J^{\overline{9}} J^{\overline{2}}\right. \\
& \left.+J^{10} J^{11} J^{10} J^{\overline{11}}+J^{10} J^{12} J^{\overline{10}} J^{\overline{12}}+J^{11} J^{12} J^{\overline{11}} J^{\overline{12}}\right)(w) \\
& + \text { other quartic, cubic, quadratic and linear terms. }
\end{aligned}
$$

The full expression of (4.49) is not presented here. As in (4.44) and (4.47), the above spin-2 current satisfies

$$
\mathrm{U}(z) W^{(2)}(w)=+\cdots, \quad F_{a}(z) W^{(2)}(w)=+\cdots, \quad a=11,12,21,22,
$$

and

$$
\left.T^{(1)}(z) W^{(2)}(w)\right|_{\frac{1}{(z-w)}}=0 .
$$

Let us consider the following OPE

$$
\begin{aligned}
\hat{G}_{21}(z) W^{(2)}(w)= & \frac{1}{(z-w)^{2}} \frac{1}{(5+k)}\left[2(2+k) \hat{G}_{21}+(-3+k) T_{+}^{\left(\frac{3}{2}\right)}\right](w) \\
& +\frac{1}{(z-w)} \frac{1}{(5+k)}\left[W_{+}^{\left(\frac{5}{2}\right)}+\frac{1}{3} \partial\left(2(2+k) \hat{G}_{21}+(-3+k) T_{+}^{\left(\frac{3}{2}\right)}\right)\right](w)+\cdots
\end{aligned}
$$

where the new spin- $\frac{5}{2}$ current is given by

$$
\begin{aligned}
W_{+}^{\left(\frac{5}{2}\right)}(w)= & \frac{2 i \sqrt{2}}{(5+k)^{4}}\left(K^{1} K^{4} K^{7} K^{\overline{1}} K^{\overline{4}}+K^{1} K^{5} K^{7} K^{\overline{1}} K^{\overline{5}}\right) \\
& + \text { other quintic, quartic, cubic, quadratic and linear terms. }
\end{aligned}
$$

The $\mathrm{U}(1)$ charges of three currents in (4.52) are the same. One can check that the spin- $\frac{5}{2}$ current commute with the subgroup currents, as in (4.50), as follows:

$$
\mathrm{U}(z) W_{+}^{\left(\frac{5}{2}\right)}(w)=+\cdots, \quad F_{a}(z) W_{+}^{\left(\frac{5}{2}\right)}(w)=+\cdots, \quad a=11,12,21,22 .
$$

Let us consider the following OPE

$$
\begin{aligned}
\hat{G}_{12}(z) W^{(2)}(w)= & \frac{1}{(z-w)^{2}} \frac{1}{(5+k)}\left[2(2+k) \hat{G}_{12}-(-3+k) T_{-}^{\left(\frac{3}{2}\right)}\right](w) \\
& +\frac{1}{(z-w)} \frac{1}{(5+k)}\left[W_{-}^{\left(\frac{5}{2}\right)}+\frac{1}{3} \partial\left(2(2+k) \hat{G}_{12}-(-3+k) T_{-}^{\left(\frac{3}{2}\right)}\right)\right](w)+\cdots
\end{aligned}
$$


where the new spin- $\frac{5}{2}$ current can be obtained

$$
\begin{aligned}
W_{-}^{\left(\frac{5}{2}\right)}(w)= & -\frac{2 i \sqrt{2}}{(5+k)^{4}}\left(K^{1} K^{4} K^{\overline{1}} K^{\overline{4}} K^{\overline{7}}+K^{1} K^{5} K^{\overline{1}} K^{\overline{5}} K^{\overline{7}}\right) \\
& + \text { other quintic, quartic, cubic, quadratic and linear terms. }
\end{aligned}
$$

The two OPEs (4.52) and (4.55) are conjugated to each other. Also one obtains the following regularity conditions (i.e. the higher spin current should commute with the subgroup currents) as in (4.54)

$$
\mathrm{U}(z) W_{-}^{\left(\frac{5}{2}\right)}(w)=+\cdots, \quad F_{a}(z) W_{-}^{\left(\frac{5}{2}\right)}(w)=+\cdots, \quad a=11,12,21,22 .
$$

Finally, the highest higher spin current of spin- $3,{ }^{51}$ can be obtained from the following OPE

$$
\begin{aligned}
\hat{G}_{21}(z) W_{-}^{\left(\frac{5}{2}\right)}(w)= & \frac{1}{(z-w)^{3}}\left[\frac{8 i(1+3 k)}{(5+k)^{2}} \hat{A}_{3}+\frac{80 i k}{3(5+k)^{2}} \hat{B}_{3}+\frac{8(-3+k)}{3(5+k)} T^{(1)}\right](w) \\
& +\frac{1}{(z-w)^{2}}\left[-\frac{4\left(15+65 k+22 k^{2}\right)}{3(3+7 k)(5+k)} \hat{T}+\frac{4(-3+k)}{3(5+k)} T^{(2)}+\frac{4(4+k)}{(5+k)} W^{(2)}\right. \\
& -\frac{16(-1+k)}{3(5+k)^{2}} \hat{A}_{1} \hat{A}_{1}-\frac{16(-1+k)}{3(5+k)^{2}} \hat{A}_{2} \hat{A}_{2}+\frac{4(13-4 k)}{3(5+k)^{2}} \hat{A}_{3} \hat{A}_{3} \\
& -\frac{4}{3(5+k)} \hat{B}_{1} \hat{B}_{1}-\frac{4}{3(5+k)} \hat{B}_{2} \hat{B}_{2}+\frac{4(-5+2 k)}{3(5+k)^{2}} \hat{B}_{3} \hat{B}_{3} \\
& \left.+\frac{4 i}{(5+k)} T^{(1)} \hat{B}_{3}+\frac{8(-4+k)}{3(5+k)^{2}} \hat{A}_{3} \hat{B}_{3}-\frac{4 i}{(5+k)} T^{(1)} \hat{A}_{3}\right](w) \\
& +\frac{1}{(z-w)}\left[\frac{1}{4} \partial\left\{\hat{G}_{21} W_{-}^{\left(\frac{5}{2}\right)}\right\}-2+\frac{24 i(1+3 k)}{(19+23 k)(5+k)}\left(\hat{T} \hat{A}_{3}-\frac{1}{2} \partial^{2} \hat{A}_{3}\right)\right. \\
& +\frac{80 i k}{(19+23 k)(5+k)}\left(\hat{T} \hat{B}_{3}-\frac{1}{2} \partial^{2} \hat{B}_{3}\right)+\frac{8(-3+k)}{(19+23 k)}\left(\hat{T} T^{(1)}-\frac{1}{2} \partial^{2} T^{(1)}\right) \\
& \left.+W^{(3)}\right](w)+\cdots,
\end{aligned}
$$

where the new spin-3 current can be described as

$$
\begin{aligned}
W^{(3)}= & -\frac{4\left(-27+23 k+2 k^{2}\right)}{5+k)^{5}(19+23 k)}\left(K^{1} K^{2} K^{4} K^{\overline{1}} K^{\overline{2}} K^{\overline{4}}+K^{1} K^{3} K^{4} K^{\overline{1}} K^{\overline{3}} K^{\overline{4}}\right) \\
& + \text { other sextic, quintic, quartic, cubic, quadratic and linear terms. }
\end{aligned}
$$

${ }^{51}$ The OPE of two quasi-primary fields, of spins $h_{i}$ and $h_{j}$ respectively, takes the form [49-55]

$$
\begin{aligned}
& \Phi_{i}(z) \Phi_{j}(w)=\frac{1}{(z-w)^{h_{i}+h_{j}}} \gamma_{i j} \\
& +\sum_{k} C_{i j k} \sum_{n=0}^{\infty} \frac{1}{(z-w)^{h_{i}+h_{j}-h_{k}-n}}\left[\frac{1}{n !} \prod_{x=0}^{n-1} \frac{\left(h_{i}-h_{j}+h_{k}+x\right)}{\left(2 h_{k}+x\right)}\right] \partial^{n} \Phi_{k}(w) .
\end{aligned}
$$

The $\gamma_{i j}$ corresponds to a metric on the space of quasi-primary fields. The structure constant $C_{i j k}$ appears in the three-point function. The index $k$ specifies all the quasi-primary fields occurring in the right hand side of (4.58). The relative coefficient functions $\left[\frac{1}{n !} \prod_{x=0}^{n-1} \frac{\left(h_{i}-h_{j}+h_{k}+x\right)}{\left(2 h_{k}+x\right)}\right]$ in the descendant fields depend on the spins and number of derivatives. For the fixed $C_{i j k}$, the relative coefficient functions in the descendant fields using (4.58) can be obtained. It is quite nontrivial to rearrange those expressions in terms of determined (and known) higher spin currents. 
Note that because the relative coefficient functions for the descendant fields of the spin-1 currents appearing in the third-order pole in (4.59) vanish, there are no descendant fields in the second-order pole. In the first-order pole in (4.59), the total derivative terms with the coefficient $\frac{1}{4}$ are descendant fields of the spin- 2 currents in the second-order pole. The remaining three terms containing $\hat{T}(w)$ are quasi-primary fields of spin-3 under the stress tensor $(3.2) .{ }^{52}$

Therefore, the higher spin currents of spins $\left(2, \frac{5}{2}, \frac{5}{2}, 3\right)$ are determined completely: (4.49), (4.53), (4.56) and (4.60). Some of the OPEs between these higher spin currents and the currents from the large $\mathcal{N}=4$ nonlinear superconformal algebra are presented in (4.48), (4.52), (4.55) and (4.59). The remaining OPEs are given in appendix B.3 and appendix C.3.

\section{Extension of large $\mathcal{N}=4$ nonlinear superconformal algebra in the coset minimal model}

In section 3 , the 11 currents of large $\mathcal{N}=4$ nonlinear superconformal algebra were constructed and in section 4, the 16 higher spin currents were found. For the extension of large $\mathcal{N}=4$ nonlinear superconformal algebra, one should calculate the OPEs between the 11 currents in section 3 and the 16 currents in section 4 as follows:

$$
\left(\begin{array}{c}
\hat{T} \\
\hat{A}_{1}, \hat{A}_{2}, \hat{A}_{3} \\
\hat{B}_{1}, \hat{B}_{2}, \hat{B}_{3} \\
\hat{G}_{11}, \hat{G}_{12}, \hat{G}_{21}, \hat{G}_{22}
\end{array}\right)(z)\left(\begin{array}{c}
T^{(1)}, T_{+}^{\left(\frac{3}{2}\right)}, T_{-}^{\left(\frac{3}{2}\right)}, T^{(2)} \\
U^{\left(\frac{3}{2}\right)}, U_{+}^{(2)}, U_{-}^{(2)}, U^{\left(\frac{5}{2}\right)} \\
V^{\left(\frac{3}{2}\right)}, V_{+}^{(2)}, V_{-}^{(2)}, V^{\left(\frac{5}{2}\right)} \\
W^{(2)}, W_{+}^{\left(\frac{5}{2}\right)}, W_{-}^{\left(\frac{5}{2}\right)}, W^{(3)}
\end{array}\right)(w) .
$$

In other words, the OPEs between 11 currents in the left hand side of (5.1) and the 16 currents in the right hand side of $(5.1)^{53}$ are needed.

\footnotetext{
${ }^{52}$ One has the following regularity conditions between the spin- 3 current and four spin- $\frac{1}{2}$ currents and spin-1 current, as in (4.57)

$$
\mathrm{U}(z) W^{(3)}(w)=+\cdots, \quad F_{a}(z) W^{(3)}(w)=+\cdots, \quad a=11,12,21,22 .
$$

Furthermore, compared to (4.51), the first-order pole between the spin- 1 current and the spin- 3 current doesn't vanish

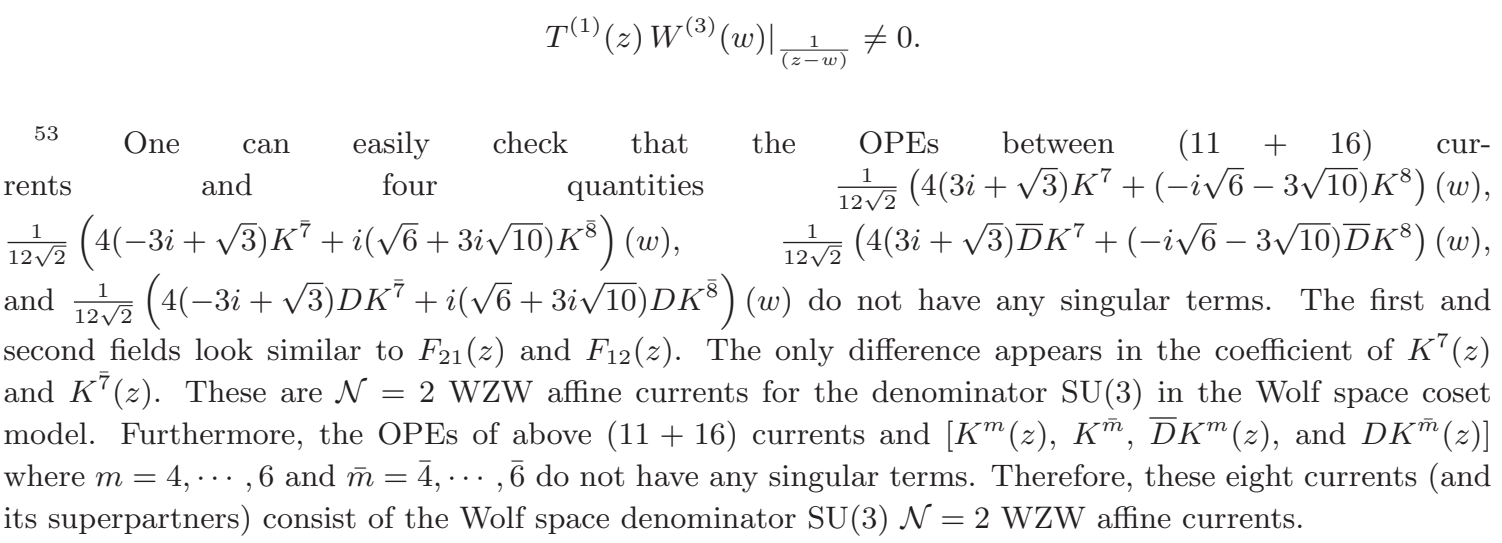




\section{$5.1 \quad \mathrm{U}(1)$ charges of higher spin currents}

It is straightforward to calculate the various $\mathrm{U}(1)$ charges under the $\mathrm{U}(1)$ current introduced in previous section. The result is as follows:

$$
\begin{aligned}
&\left.\left(-2 i \gamma_{A} \hat{A}_{3}-2 i \gamma_{B} \hat{B}_{3}\right)(z) T^{(1)}(w)\right|_{\frac{1}{(z-w)}}=0 \\
&\left.\left(-2 i \gamma_{A} \hat{A}_{3}-2 i \gamma_{B} \hat{B}_{3}\right)(z) T_{ \pm}^{\left(\frac{3}{2}\right)}(w)\right|_{\frac{1}{(z-w)}}=\frac{1}{(z-w)}\left[ \pm \frac{(3+k)}{(5+k)}\right] T_{ \pm}^{\left(\frac{3}{2}\right)}(w) \\
&\left.\left(-2 i \gamma_{A} \hat{A}_{3}-2 i \gamma_{B} \hat{B}_{3}\right)(z) T^{(2)}(w)\right|_{\frac{1}{(z-w)}}=0 \\
&\left.\left(-2 i \gamma_{A} \hat{A}_{3}-2 i \gamma_{B} \hat{B}_{3}\right)(z)\left(\begin{array}{l}
U^{\left(\frac{3}{2}\right)} \\
V^{\left(\frac{3}{2}\right)}
\end{array}\right)(w)\right|_{\frac{1}{(z-w)}}=\frac{1}{(z-w)}\left[ \pm \frac{(-3+k)}{(5+k)}\right]\left(\begin{array}{l}
U^{\left(\frac{3}{2}\right)} \\
V^{\left(\frac{3}{2}\right)}
\end{array}\right)(w), \\
&\left.\left(-2 i \gamma_{A} \hat{A}_{3}-2 i \gamma_{B} \hat{B}_{3}\right)(z)\left(\begin{array}{l}
U_{+}^{(2)} \\
V_{-}^{(2)}
\end{array}\right)(w)\right|_{\frac{1}{(z-w)}}=\frac{1}{(z-w)}\left[ \pm \frac{2 k}{(5+k)}\right]\left(\begin{array}{l}
U_{+}^{(2)} \\
V_{-}^{(2)}
\end{array}\right)(w) \\
&\left.\left(-2 i \gamma_{A} \hat{A}_{3}-2 i \gamma_{B} \hat{B}_{3}\right)(z)\left(\begin{array}{l}
U_{-}^{(2)} \\
V_{+}^{(2)}
\end{array}\right)(w)\right|_{\frac{1}{(z-w)}}=\frac{1}{(z-w)}\left[\mp \frac{6}{(5+k)}\right]\left(\begin{array}{l}
U_{-}^{(2)} \\
V_{+}^{(2)}
\end{array}\right)(w), \\
&\left.\left(-2 i \gamma_{A} \hat{A}_{3}-2 i \gamma_{B} \hat{B}_{3}\right)(z)\left(\begin{array}{l}
U^{\left(\frac{5}{2}\right)} \\
V^{\left(\frac{5}{2}\right)}
\end{array}\right)(w)\right|_{\frac{1}{(z-w)}}=\frac{1}{(z-w)}\left[ \pm \frac{(-3+k)}{(5+k)}\right]\left(\begin{array}{l}
U^{\left(\frac{5}{2}\right)} \\
V^{\left(\frac{5}{2}\right)}
\end{array}\right)(w) \\
&\left.\left(-2 i \gamma_{A} \hat{A}_{3}-2 i \gamma_{B} \hat{B}_{3}\right)(z) W^{(2)}(w)\right|_{\frac{1}{(z-w)}}=0 \\
&\left.\left(-2 i \gamma_{A} \hat{A}_{3}-2 i \gamma_{B} \hat{B}_{3}\right)(z) W_{ \pm}^{\left(\frac{5}{2}\right)}(w)\right|_{\frac{1}{(z-w)}}=\frac{1}{(z-w)}\left[ \pm \frac{(3+k)}{(5+k)}\right] W_{ \pm}^{\left(\frac{5}{2}\right)}(w) \\
&\left.\left(-2 i \gamma_{A} \hat{A}_{3}-2 i \gamma_{B} \hat{B}_{3}\right)(z) W^{(3)}(w)\right|_{\frac{1}{(z-w)}}=0
\end{aligned}
$$

We present these $\mathrm{U}(1)$ charges in the table 2 explicitly. ${ }^{54}$ Then the $\mathrm{U}(1)$ charges of all the composite fields coming from the $(11+16)$ currents can be determined by the assignments in table 1 and table 2. The necessary $\mathrm{U}(1)$ assignments in this paper are presented in table 3-table 7.

\section{$5.2 \quad$ Structure of OPEs}

The 16 higher spin currents are primary fields under the stress energy tensor $\hat{T}(z)(3.2)$. Then the remaining nontrivial OPEs between the 16 currents and the 11 currents are given by 1) the OPEs between the six spin- 1 currents and the 16 higher spin currents described in appendix B and 2) the OPEs between the four spin- $\frac{3}{2}$ currents and the 16 higher spin currents described in appendix $\mathrm{C}$. It is nontrivial to extract all the structures in the right hand sides of above OPEs. Because the left hand sides of these OPEs are known in terms $\mathcal{N}=2$ WZW affine currents, we would like to express them in terms of known currents. If one cannot write them with the known currents, then one should obtain the new primary fields appearing in the right hand sides along the line of footnote 51. One should write down

\footnotetext{
${ }^{54}$ If one uses the notations $(++,+-,-+,--)$ for the fermion currents and $(+,-, 3)$ for the bosonic currents which tansform as triplet under the $\mathrm{SU}(2)$, then the expression (5.2) will be simpler.
} 
the correct terms, which preserve the right $\mathrm{U}(1)$ charge described before via tables $1-7$, with arbitrary coefficients at the specific pole (with fixed spin) in the given OPE.

For example, in the OPEs between the spin- 1 currents of large $\mathcal{N}=4$ nonlinear algebra and the largest spin-3 higher spin current, by dimensional analysis of spin, the first-order pole contains the spin-3 fields in appendices (B.29)-(B.32). One can rearrange these OPEs in terms of descendant fields with known coefficients and (quasi) primary fields at first-order pole terms. The $\mathrm{U}(1)$ charges of composite spin-3 fields are presented in table 5 . The $\mathrm{U}(1)$ charge of the left hand side of appendix (B.29) is the sum of the $\mathrm{U}(1)$ charge of $\left(\hat{A}_{1}+i \hat{A}_{2}\right)(z)$ and the one of $W^{(3)}(w)$. The former is given by $-\frac{6}{(5+k)}$ and the latter is given by 0 from table 2. Therefore, the total $\mathrm{U}(1)$ charge, $-\frac{6}{(5+k)}$, should appear in the right hand side of appendix (B.29). Then from the first row of table 5, there exist 26 possible composite spin-3 fields with $\mathrm{U}(1)$ charge $-\frac{6}{(5+k)}$. Because the left hand side of appendix (B.29) is written in terms of $\mathcal{N}=2$ WZW affine currents explicitly, let us subtract the above 26 terms with arbitrary $k$-dependent coefficients from the left hand side of appendix (B.29). In order to vanish these quantities, one should solve this equation. This is equivalent to solve many linear equations with respect to the above undetermined $k$-dependent coefficients. It turns out that the unknown coefficient functions can be obtained completely and they are given in appendix (B.29). Of course, when the order of any product is changed, the extra derivative terms arise either in table 6 or in the OPE of appendix (B.29).

What about the OPEs between the spin- $\frac{3}{2}$ currents of large $\mathcal{N}=4$ nonlinear superconformal algebra and the above spin-3 higher spin current? They are presented in appendix (C.43)-(C.46). One can have the composite spin- $\frac{7}{2}$ fields in the first-order pole of appendix (C.43) by dimensional analysis as before. The total U(1) charge of this OPE should be preserved. Because the U(1) charge of the left hand side is given by $\frac{(-3+k)}{5+k}$ from the table 2 , the possible 68 composite fields with this $\mathrm{U}(1)$ charge are presented in the first row of table 7. As performed in previous paragraph, it turns out that the unknown 41 coefficient functions can be obtained completely and they are given in appendix (C.43).

In these examples, there are no new (quasi) primary fields in the first-order pole. As in (4.59), when one cannot solve the linear equations completely due to the appearance of new (quasi) primary fields, one should resort to the procedure described in the footnote 51. That is, one should check that any singular terms consist of a couple of descendant fields (with determined coefficient functions using the formula in the footnote 51) and a couple of (quasi) primary fields.

As in an abstract, the result from appendices $\mathrm{B}$ and $\mathrm{C}$ shows that the right hand sides of all the OPEs contains the composite fields which can be obtained from the known $(11+16)$ currents. There exist no new primary fields. One expects that the new primary fields will appear in the OPEs between the 16 currents and themselves [48].

\section{Conclusions and outlook}

As in an abstract, the 16 higher spin currents given in (1.2) and 11 currents of large $\mathcal{N}=4$ nonlinear algebra are found explicitly in the Wolf space coset model $\frac{\mathrm{SU}(5)}{\mathrm{SU}(3) \times \mathrm{SU}(2) \times \mathrm{U}(1)}$. Part of this extended large $\mathcal{N}=4$ nonlinear algebra are described in appendices $\mathrm{A}, \mathrm{B}$ and $\mathrm{C}$. 


\begin{tabular}{|c|c|c|}
\hline $\mathrm{U}(1)$ charge & 16 higher spin currents & 11 currents in table 1 \\
\hline$\frac{2 k}{(5+k)}$ & $U_{+}^{(2)}$ & $\hat{B}_{-}$ \\
\hline$\frac{(3+k)}{(5+k)}$ & $T_{+}^{\left(\frac{3}{2}\right)}, \quad W_{+}^{\left(\frac{5}{2}\right)}$ & $\hat{G}_{21}$ \\
\hline$\frac{(-3+k)}{(5+k)}$ & $U^{\left(\frac{3}{2}\right)}, \quad U^{\left(\frac{5}{2}\right)}$ & $\hat{G}_{11}$ \\
\hline$\frac{6}{(5+k)}$ & $V_{+}^{(2)}$ & $\hat{A}_{-}$ \\
\hline 0 & $T^{(1)}, \quad T^{(2)}, \quad W^{(2)}, \quad W^{(3)}$ & $\hat{A}_{3}$ \\
\hline$-\frac{6}{(5+k)}$ & $U_{-}^{(2)}$ & $\hat{A}_{+}$ \\
\hline$-\frac{(-3+k)}{(5+k)}$ & $V^{\left(\frac{3}{2}\right)}, \quad V^{\left(\frac{5}{2}\right)}$ & $\hat{G}_{22}$ \\
\hline$-\frac{(3+k)}{(5+k)}$ & $T_{-}^{\left(\frac{3}{2}\right)}, \quad W_{-}^{\left(\frac{5}{2}\right)}$ & $\hat{G}_{12}$ \\
\hline$-\frac{2 k}{(5+k)}$ & $V_{-}^{(2)}$ & $\hat{B}_{+}$ \\
\hline
\end{tabular}

Table 2. The $\mathrm{U}(1)$ charges for the 16 currents from (5.2). For convenience, the $\mathrm{U}(1)$ charges for the 11 currents of large $\mathcal{N}=4$ nonlinear superconformal algebra in table 1 are presented also. The fields appearing in the first-order poles of (B.4), (B.5), [(B.1) and (C.1)], (B.3), (B.9), (B.3), (B.2), [(B.6) and (C.2)] and (B.4) correspond to the above ones located at each row, respectively.

\begin{tabular}{|c|c|}
\hline $\mathrm{U}(1)$ charge & Composite fields of spin-2 \\
\hline$\frac{2(3+k)}{(5+k)}$ & $\hat{A}_{-} \hat{B}_{-}$ \\
\hline$\frac{2 k}{(5+k)}$ & $T^{(1)} \hat{B}_{-}, \quad U_{+}^{(2)}, \quad \partial \hat{B}_{-}, \quad \hat{A}_{3} \hat{B}_{-}, \quad \hat{B}_{-} \hat{B}_{3}$ \\
\hline$\frac{2(-3+k)}{(5+k)}$ & $\hat{A}_{+} \hat{B}_{-}$ \\
\hline$\frac{6}{(5+k)}$ & $T^{(1)} \hat{A}_{-}, \quad V_{+}^{(2)}, \quad \partial \hat{A}_{-}, \quad \hat{A}_{-} \hat{A}_{3}, \quad \hat{A}_{-} \hat{B}_{3}$ \\
\hline 0 & 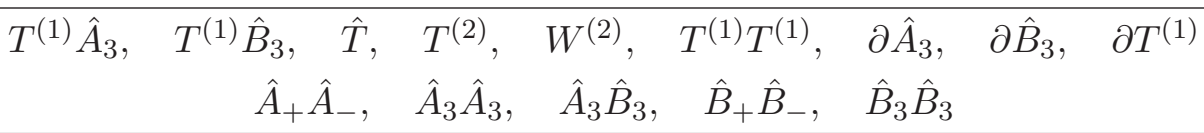 \\
\hline$-\frac{6}{(5+k)}$ & $T^{(1)} \hat{A}_{+}, \quad U_{-}^{(2)}, \quad \partial \hat{A}_{+}, \quad \hat{A}_{+} \hat{A}_{3}, \quad \hat{A}_{+} \hat{B}_{3}$ \\
\hline$-\frac{2(-3+k)}{(5+k)}$ & $\hat{A}_{-} \hat{B}_{+}$ \\
\hline$-\frac{2 k}{(5+k)}$ & $T^{(1)} \hat{B}_{+}, \quad V_{-}^{(2)}, \quad \partial \hat{B}_{+}, \quad \hat{A}_{3} \hat{B}_{+}, \quad \hat{B}_{+} \hat{B}_{3}$ \\
\hline$-\frac{2(3+k)}{(5+k)}$ & $\hat{A}_{+} \hat{B}_{+}$ \\
\hline
\end{tabular}

Table 3. The U(1) charges for the spin-2 fields which can be obtained from the U(1) charges in table 2. The fields appearing in the first-order pole in [(B.8) and (C.5)], [(C.3) and (C.12)], [(B.7), (B.12) and (C.10)], (C.6), [(B.10), (C.4) and (C.13)], [(B.21) and (C.11)], (B.8), (B.22) and (B.11) correspond to the above ones respectively. One sees that the fields located at the first four rows have their conjugated ones in the last four rows.

If one says the last statement of the abstract precisely, the $(11+16)$ currents commute with the $\mathcal{N}=2$ (effectively $\mathcal{N}=1$ ) WZW affine currents living in the $\mathcal{N}=1$ subgroup $\mathrm{SU}(3) \times \mathrm{U}(1)$ and the bosonic subgroup $\mathrm{SU}(2)$. The regular conditions of these currents with the four spin- $\frac{1}{2}$ currents and spin-1 current were very crucial. Three of four spin- $\frac{1}{2}$ currents live in the above bosonic subgroup $\mathrm{SU}(2)$ while one of them and spin-1 current 


\begin{tabular}{|c|c|}
\hline $\mathrm{U}(1)$ charge & Composite fields of spin- $\frac{5}{2}$ \\
\hline$\frac{3(1+k)}{(5+k)}$ & $\hat{B}_{-} \hat{G}_{21}, \quad \hat{B}_{-} T_{+}^{\left(\frac{3}{2}\right)}$ \\
\hline$\frac{3(-1+k)}{(5+k)}$ & $\hat{B}_{-} \hat{G}_{11}, \quad \hat{B}_{-} U^{\left(\frac{3}{2}\right)}$ \\
\hline$\frac{(9+k)}{(5+k)}$ & $\hat{A}_{-} \hat{G}_{21}, \quad \hat{A}_{-} T_{+}^{\left(\frac{3}{2}\right)}$ \\
\hline$\frac{(3+k)}{(5+k)}$ & $\begin{array}{c}W_{+}^{\left(\frac{5}{2}\right)}, \quad \hat{A}_{-} \hat{G}_{11}, \quad \hat{A}_{-} U^{\frac{3}{2}}, \quad \hat{A}_{3} \hat{G}_{21}, \quad \hat{A}_{3} T_{+}^{\left(\frac{3}{2}\right)}, \quad \hat{B}_{-} \hat{G}_{22} \\
\hat{B}_{-} V^{\frac{3}{2}}, \quad \hat{B}_{3} \hat{G}_{21}, \quad \hat{B}_{3} T_{+}^{\left(\frac{3}{2}\right)}, \quad T^{(1)} \hat{G}_{21}, \quad T^{(1)} T_{+}^{\left(\frac{3}{2}\right)}, \quad \partial \hat{G}_{21}, \quad \partial T_{+}^{\left(\frac{3}{2}\right)}\end{array}$ \\
\hline$\frac{(-3+k)}{(5+k)}$ & $\begin{array}{c}\hat{A}_{+} \hat{G}_{21}, \quad \hat{A}_{+} T_{+}^{\left(\frac{3}{2}\right)}, \quad U^{\left(\frac{5}{2}\right)}, \quad \hat{A}_{3} \hat{G}_{11}, \quad \hat{A}_{3} U^{\left(\frac{3}{2}\right)}, \quad \hat{B}_{-} \hat{G}_{12} \\
\hat{B}_{-} T_{-}^{\left(\frac{3}{2}\right)}, \quad \hat{B}_{3} \hat{G}_{11}, \quad \hat{B}_{3} U^{\left(\frac{3}{2}\right)}, \quad T^{(1)} \hat{G}_{11}, \quad T^{(1)} U^{\left(\frac{3}{2}\right)}, \quad \partial \hat{G}_{11}, \quad \partial U^{\left(\frac{3}{2}\right)}\end{array}$ \\
\hline$\frac{(-9+k)}{(5+k)}$ & $\hat{A}_{+} \hat{G}_{11}, \quad \hat{A}_{+} U^{\left(\frac{3}{2}\right)}$ \\
\hline$-\frac{(-9+k)}{(5+k)}$ & $\hat{A}_{-} \hat{G}_{22}, \quad \hat{A}_{-} V^{\left(\frac{3}{2}\right)}$ \\
\hline$-\frac{(-3+k)}{(5+k)}$ & 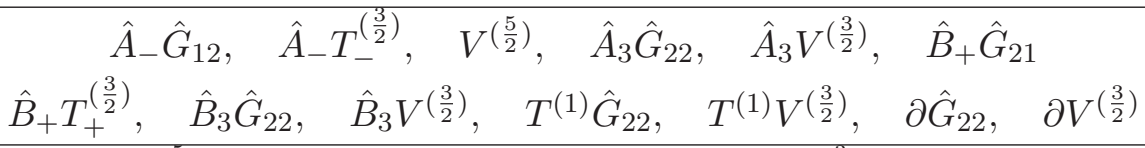 \\
\hline$-\frac{(3+k)}{(5+k)}$ & $\begin{array}{c}W_{-}^{\left(\frac{5}{2}\right)}, \quad \hat{A}_{+} \hat{G}_{22}, \quad \hat{A}_{+} V^{\frac{3}{2}}, \quad \hat{A}_{3} \hat{G}_{12}, \quad \hat{A}_{3} T_{-}^{\left(\frac{3}{2}\right)}, \quad \hat{B}_{+} \hat{G}_{11} \\
\hat{B}_{+} U^{\frac{3}{2}}, \quad \hat{B}_{3} \hat{G}_{12}, \quad \hat{B}_{3} T_{-}^{\left(\frac{3}{2}\right)}, \quad T^{(1)} \hat{G}_{12}, \quad T^{(1)} T_{-}^{\left(\frac{3}{2}\right)}, \quad \partial \hat{G}_{12}, \quad \partial T_{-}^{\left(\frac{3}{2}\right)}\end{array}$ \\
\hline$-\frac{(9+k)}{(5+k)}$ & $\hat{A}_{+} \hat{G}_{12}, \quad \hat{A}_{+} T_{-}^{\left(\frac{3}{2}\right)}$ \\
\hline$-\frac{3(-1+k)}{(5+k)}$ & $\hat{B}_{+} V^{\left(\frac{3}{2}\right)}$ \\
\hline$-\frac{3(1+k)}{(5+k)}$ & $\hat{B}_{+} \hat{G}_{12}, \quad \hat{B}_{+} T_{-}^{\left(\frac{3}{2}\right)}$ \\
\hline
\end{tabular}

Table 4. The $\mathrm{U}(1)$ charges for the spin- $\frac{5}{2}$ fields which can be obtained from the $\mathrm{U}(1)$ charges in table 2. The fields appearing in the first-order pole in (C.16), (C.14), (C.19), (C.17), [(C.7), (C.15), (C.20) and (C.33)], (C.18), (C.18), [(C.9), (C.27) and (C.28)], [(C.8), (C.21) and (C.34)], (C.19), (C.14) and (C.16) correspond to the above ones respectively. Similarly, the fields appearing in the first-order pole in (B.26), (B.16), (B.24), [(B.14) and (B.20)], [(B.23) and (B.28)], (B.13), (B.18), [(B.25) and (B.27)], [(B.15) and (B.17)], (B.24), (B.19) and (B.26) correspond to the above ones respectively. The fields in the first six rows are conjugated to each other in those in the last six rows.

live in the $\mathcal{N}=1$ subgroup $\mathrm{U}(1)$. The two $\mathrm{SU}(2)$ affine algebras are embedded in the $\mathcal{N}=4$ coset theory in nontrivial way. The $\mathrm{SU}(2)_{3}$ algebra is realized by the Wolf space coset currents while the $\mathrm{SU}(2)_{k}$ algebra is realized by two currents from the Wolf space subgroup and one remaining current from both Wolf space subgroup and Wolf space coset.

Compared to the work of [35] where the $\mathcal{N}=2 \mathcal{W}_{5}$ algebra with spin contents of $\left(1, \frac{3}{2}, \frac{3}{2}, 2\right),\left(2, \frac{5}{2}, \frac{5}{2}, 3\right),\left(3, \frac{7}{2}, \frac{7}{2}, 4\right)$ and $\left(4, \frac{9}{2}, \frac{9}{2}, 5\right)$ is realized on the coset $\mathbf{C P}^{4}=\frac{\mathrm{SU}(5)}{\mathrm{SU}(4) \times \mathrm{U}(1)}$, the present Wolf space coset model has more higher spin currents in the sense that there exist the additional second and third multiplets of (1.2). This is because the dimension of the subgroup in Wolf space coset is less than the one of the above $\mathbf{C P}^{4}$ model and there exists more room for construction of higher spin currents.

From the OPEs between the 11 currents and 16 higher spin currents, one realizes that some of the OPEs have special features. Let us consider the OPEs between four spin- $\frac{3}{2}$ 


\begin{tabular}{|c|c|}
\hline $\mathrm{U}(1)$ charge & Composite fields of spin-3 \\
\hline$\frac{(6+2 k)}{(5+k)}$ & $\begin{array}{c}\hat{A}_{3} \hat{A}_{-} \hat{B}_{-}, \hat{B}_{3} \hat{A}_{-} \hat{B}_{-}, T^{(1)} \hat{A}_{-} \hat{B}_{-}, \hat{A}_{-} U_{+}^{(2)}, \hat{A}_{-} \partial \hat{B}_{-}, \hat{B}_{-} V_{+}^{(2)}, \hat{B}_{-} \partial \hat{A}_{-}, \\
\hat{G}_{21} \hat{G}_{21}, \hat{G}_{21} T_{+}^{\left(\frac{3}{2}\right)}, T_{+}^{\left(\frac{3}{2}\right)} T_{+}^{\left(\frac{3}{2}\right)},\end{array}$ \\
\hline$\frac{2 k}{(5+k)}$ & $\begin{array}{c}T^{(1)} U_{+}^{(2)}, T^{(1)} \partial \hat{B}_{-}, \hat{A}_{3} U_{+}^{(2)}, \hat{A}_{3} \partial \hat{B}_{-}, \hat{B}_{-} \hat{T}, \hat{B}_{-} T^{(2)}, \hat{B}_{-} W^{(2)}, \hat{B}_{-} \partial \hat{A}_{3}, \hat{B}_{-} \partial \hat{B}_{3}, \\
\hat{B}_{-} \partial T^{(1)}, \hat{B}_{3} U_{+}^{(2)}, \hat{B}_{3} \partial \hat{B}_{-}, \partial U_{+}^{(2)}, \partial^{2} \hat{B}_{-}, \hat{G}_{21} \hat{G}_{11}, \hat{G}_{21} U^{\left(\frac{3}{2}\right)}, \hat{G}_{11} T_{+}^{\left(\frac{3}{2}\right)}, \\
T_{+}^{\left(\frac{3}{2}\right)} U^{\left(\frac{3}{2}\right)}, \hat{A}_{-} \hat{A}_{+} \hat{B}_{-}, \hat{A}_{3} \hat{A}_{3} \hat{B}_{-}, \hat{A}_{3} \hat{B}_{3} \hat{B}_{-}, \hat{B}_{-} \hat{B}_{-} \hat{B}_{+}, \hat{B}_{3} \hat{B}_{3} \hat{B}_{-}, T^{(1)} \hat{A}_{3} \hat{B}_{-}, \\
T^{(1)} \hat{B}_{3} \hat{B}_{-}, T^{(1)} T^{(1)} \hat{B}_{-},\end{array}$ \\
\hline$\frac{(-6+2 k)}{(5+k)}$ & $\begin{array}{c}\hat{A}_{3} \hat{A}_{+} \hat{B}_{-}, \hat{B}_{3} \hat{A}_{+} \hat{B}_{-}, T^{(1)} \hat{A}_{+} \hat{B}_{-}, \hat{B}_{-} U_{-}^{(2)}, \hat{B}_{-} \partial \hat{A}_{+}, \hat{A}_{+} U_{+}^{(2)}, \hat{A}_{+} \partial \hat{B}_{-} \\
\hat{G}_{11} \hat{G}_{11}, \hat{G}_{11} U^{\left(\frac{3}{2}\right)}, U^{\left(\frac{3}{2}\right)} U^{\left(\frac{3}{2}\right)}\end{array}$ \\
\hline$\frac{6}{(5+k)}$ & $\begin{array}{c}T^{(1)} V_{+}^{(2)}, T^{(1)} \partial \hat{A}_{-}, \hat{A}_{-} \hat{T}_{,} \hat{A}_{-} T^{(2)}, \hat{A}_{-} W^{(2)}, \hat{A}_{-} \partial \hat{A}_{3}, \hat{A}_{-} \partial \hat{B}_{3}, \hat{A}_{-} \partial T^{(1)}, \\
\hat{A}_{3} V_{+}^{(2)}, \partial V_{+}^{(2)}, \partial^{2} \hat{A}_{-}, \hat{G}_{21} \hat{G}_{22}, \hat{G}_{21} V^{\left(\frac{3}{2}\right)}, \hat{G}_{22} T_{+}^{\left(\frac{3}{2}\right)}, T_{+}^{\left(\frac{3}{2}\right)} V^{\left(\frac{3}{2}\right)}, \hat{A}_{-} \hat{A}_{+} \hat{A}_{-}, \\
\hat{A}_{-} \hat{A}_{3} \hat{A}_{3}, \hat{A}_{-} \hat{A}_{3} \hat{B}_{3}, \hat{A}_{-} \hat{B}_{+} \hat{B}_{-}, \hat{A}_{-} \hat{B}_{3} \hat{B}_{3}, \hat{B}_{3} V_{+}^{(2)}, \hat{A}_{-} T^{(1)} \hat{A}_{3}, \hat{A}_{-} T^{(1)} \hat{B}_{3}, \\
\hat{A}_{-} T^{(1)} T^{(1)}, \hat{A}_{3} \partial \hat{A}_{-}, \hat{B}_{3} \partial \hat{A}_{-}\end{array}$ \\
\hline 0 & $\begin{array}{c}W^{(3)}, T^{(1)} \hat{T}, T^{(1)} T^{(2)}, T^{(1)} W^{(2)}, T^{(1)} \partial \hat{A}_{3}, T^{(1)} \partial \hat{B}_{3}, T^{(1)} \partial T^{(1)}, T^{(1)} T^{(1)} T^{(1)}, \\
\hat{A}_{+} V_{+}^{(2)}, \hat{A}_{+} \partial \hat{A}_{-}, \hat{A}_{-} U_{-}^{(2)}, \hat{A}_{-} \partial \hat{A}_{+}, \hat{A}_{3} \hat{T}, \hat{A}_{3} T^{(2)}, \hat{A}_{3} W^{(2)}, T_{+}^{\left(\frac{3}{2}\right)} T_{-}^{\left(\frac{3}{2}\right)} \\
\hat{A}_{3} \partial \hat{A}_{3}, \hat{A}_{3} \partial \hat{B}_{3}, \hat{A}_{3} \partial T^{(1)}, \hat{B}_{+} U_{+}^{(2)}, \hat{B}_{+} \partial \hat{B}_{-}, \hat{B}_{-} V_{-}^{(2)}, \hat{B}_{-} \partial \hat{B}_{+}, \hat{B}_{3} \hat{T}, \hat{B}_{3} T^{(2)}, \\
\hat{B}_{3} W^{(2)}, \hat{B}_{3} \partial \hat{A}_{3}, \hat{B}_{3} \partial \hat{B}_{3}, \hat{B}_{3} \partial T^{(1)}, \partial \hat{T}^{(2)} \partial T^{(2)}, \partial W^{(2)}, T^{(1)} \partial T^{(1)}, \partial^{2} \hat{A}_{3}, \partial^{2} \hat{B}_{3}, \partial^{2} T^{(1)}, \\
\partial T^{(1)} \hat{A}_{3}, \partial T^{(1)} \hat{B}_{3}, \hat{G}_{11} \hat{G}_{22}, \hat{G}_{11} V^{\left(\frac{3}{2}\right)}, \hat{G}_{12} \hat{G}_{21}, \hat{G}_{12} T_{+}^{\left(\frac{3}{2}\right)}, \hat{G}_{21} T_{-}^{\left(\frac{3}{2}\right)}, \hat{G}_{22} U^{\left(\frac{3}{2}\right)}, U^{\left(\frac{3}{2}\right)} V^{\left(\frac{3}{2}\right)}, \\
\hat{A}_{+} \hat{A}_{-} \hat{A}_{3}, \hat{A}_{+} \hat{A}_{-} \hat{B}_{3}, \hat{A}_{3} \hat{A}_{3} \hat{A}_{3}, \hat{A}_{3} \hat{A}_{3} \hat{B}_{3}, \hat{A}_{3} \hat{B}_{3} \hat{B}_{3}, \hat{B}_{+} \hat{B}_{-} \hat{B}_{3}, \hat{B}_{+} \hat{B}_{-} \hat{A}_{3}, \hat{B}_{3} \hat{B}_{3} \hat{B}_{3}, \\
T^{(1)} \hat{A}_{+} \hat{A}_{-}, T^{(1)} \hat{A}_{3} \hat{A}_{3}, T^{(1)} \hat{A}_{3} \hat{B}_{3}, T^{(1)} \hat{B}_{+} \hat{B}_{-}, T^{(1)} \hat{B}_{3} \hat{B}_{3}, T^{(1)} T^{(1)} \hat{A}_{3}, T^{(1)} T^{(1)} \hat{B}_{3},\end{array}$ \\
\hline$-\frac{6}{(5+k)}$ & $\begin{array}{c}T^{(1)} U_{-}^{(2)}, T^{(1)} \partial \hat{A}_{+}, \hat{A}_{+} \hat{T}, \hat{A}_{+} T^{(2)}, \hat{A}_{+} W^{(2)}, \hat{A}_{+} \partial \hat{A}_{3}, \hat{A}_{+} \partial \hat{B}_{3}, \hat{A}_{+} \partial T^{(1)} \\
\hat{A}_{3} U_{-}^{(2)}, \partial U_{-}^{(2)}, \partial^{2} \hat{A}_{+}, \hat{G}_{12} \hat{G}_{11}, \hat{G}_{12} U^{\left(\frac{3}{2}\right)}, \hat{G}_{11} T_{-}^{\left(\frac{3}{2}\right)}, T_{-}^{\left(\frac{3}{2}\right)} U^{\left(\frac{3}{2}\right)}, \hat{A}_{+} \hat{A}_{-} \hat{A}_{+} \\
\hat{A}_{+} \hat{A}_{3} \hat{A}_{3}, \hat{A}_{+} \hat{A}_{3} \hat{B}_{3}, \hat{A}_{+} \hat{B}_{-} \hat{B}_{+}, \hat{A}_{+} \hat{B}_{3} \hat{B}_{3}, \hat{B}_{3} U_{-}^{(2)} \\
\hat{A}_{+} T^{(1)} \hat{A}_{3}, \hat{A}_{+} T^{(1)} \hat{B}_{3}, \hat{A}_{+} T^{(1)} T^{(1)}, \hat{A}_{3} \partial \hat{A}_{+}, \hat{B}_{3} \partial \hat{A}_{+}\end{array}$ \\
\hline$-\frac{(-6+2 k)}{(5+k)}$ & $\begin{aligned} & \hat{A}_{3} \hat{A}_{-} \hat{B}_{+}, \hat{B}_{3} \hat{A}_{-} \hat{B}_{+}, T^{(1)} \hat{A}_{-} \hat{B}_{+}, \hat{B}_{+} V_{+}^{(2)}, \hat{B}_{+} \partial \hat{A}_{-} \\
& \hat{A}_{-} V_{-}^{(2)}, \hat{A}_{-} \partial \hat{B}_{+}, \hat{G}_{22} \hat{G}_{22}, \hat{G}_{22} V^{\left(\frac{3}{2}\right)}, V^{\left(\frac{3}{2}\right)} V^{\left(\frac{3}{2}\right)}\end{aligned}$ \\
\hline$-\frac{2 k}{(5+k)}$ & $\begin{array}{c}T^{(1)} V_{-}^{(2)}, T^{(1)} \partial \hat{B}_{+}, \hat{A}_{3} V_{-}^{(2)}, \hat{A}_{3} \partial \hat{B}_{+}, \hat{B}_{+} T^{(2)}, \hat{B}_{+} W^{(2)}, \hat{B}_{+} \partial \hat{A}_{3}, \hat{B}_{+} \partial \hat{B}_{3}, \hat{B}_{+} \partial T^{(1)}, \\
\hat{B}_{3} V_{-}^{(2)}, \hat{B}_{+} \hat{T}, \hat{B}_{3} \partial \hat{B}_{+}, \partial V_{-}^{(2)}, \hat{G}_{12} \hat{G}_{22}, \hat{G}_{12} V^{\left(\frac{3}{2}\right)}, \hat{G}_{22} T_{-}^{\left(\frac{3}{2}\right)}, T_{-}^{\left(\frac{3}{2}\right)} V^{\left(\frac{3}{2}\right)}, \hat{A}_{+} \hat{A}_{-} \hat{B}_{+}, \\
\hat{A}_{3} \hat{A}_{3} \hat{B}_{+}, \partial^{2} \hat{B}_{+}, \hat{A}_{3} \hat{B}_{3} \hat{B}_{+}, \hat{B}_{+} \hat{B}_{+} \hat{B}_{-}, \hat{B}_{3} \hat{B}_{3} \hat{B}_{+}, T^{(1)} \hat{A}_{3} \hat{B}_{+}, T^{(1)} \hat{B}_{3} \hat{B}_{+}, T^{(1)} T^{(1)} \hat{B}_{+},\end{array}$ \\
\hline$-\frac{(6+2 k)}{(5+k)}$ & $\begin{array}{c}\hat{A}_{3} \hat{A}_{+} \hat{B}_{+}, \hat{B}_{3} \hat{A}_{+} \hat{B}_{+}, T^{(1)} \hat{A}_{+} \hat{B}_{+}, \hat{A}_{+} V_{-}^{(2)}, \hat{A}_{+} \partial \hat{B}_{+} \\
\hat{B}_{+} U_{-}^{(2)}, \hat{B}_{+} \partial \hat{A}_{+}, \hat{G}_{12} \hat{G}_{12}, \hat{G}_{12} T_{-}^{\left(\frac{3}{2}\right)}, T_{-}^{\left(\frac{3}{2}\right)} T_{-}^{\left(\frac{3}{2}\right)}\end{array}$ \\
\hline
\end{tabular}

Table 5. The U(1) charges for the spin-3 fields which can be obtained from the U(1) charges in table 2. The fields appearing in the first-order pole in appendix (C.37), [(C.24) and (C.35)], (C.22), [(B.30), (C.31) and (C.38)], [(C.25), (C.29), (C.36) and (C.41)], [(B.29), (C.23) and (C.39)], (C.32), $[(\mathrm{B} .31),(\mathrm{C} .30)$ and $(\mathrm{C} .42)]$ and $(\mathrm{C} .40)$ correspond to the above ones respectively. 


\begin{tabular}{|c|c|}
\hline $\mathrm{U}(1)$ charge & Composite fields of spin- $\frac{7}{2}$ \\
\hline$\frac{(3+k)}{(5+k)}$ & $\begin{array}{c}\hat{A}_{-} \hat{A}_{+} \hat{G}_{21}, \hat{A}_{-} \hat{A}_{+} T_{+}^{\left(\frac{3}{2}\right)}, \hat{A}_{-} U^{\left(\frac{5}{2}\right)}, \hat{A}_{-} \hat{A}_{3} \hat{G}_{11}, \hat{A}_{-} \hat{A}_{3} U^{\left(\frac{3}{2}\right)}, \hat{A}_{-} \hat{B}_{-} \hat{G}_{12}, \\
\hat{A}_{-} \hat{B}_{-} T_{-}^{\left(\frac{3}{2}\right)}, \hat{A}_{-} \hat{B}_{3} \hat{G}_{11}, \hat{A}_{-} \hat{B}_{3} U^{\left(\frac{3}{2}\right)}, \hat{A}_{-} \partial \hat{G}_{11}, \hat{A}_{-} \partial U^{\left(\frac{3}{2}\right)}, \hat{B}_{+} \hat{B}_{-} \hat{G}_{21}, \hat{B}_{+} \hat{B}_{-} T_{+}^{\left(\frac{3}{2}\right)}, \\
\hat{B}_{-} V^{\left(\frac{5}{2}\right)}, \hat{B}_{-} \hat{A}_{3} \hat{G}_{22}, \hat{B}_{-} \hat{A}_{3} V^{\left(\frac{3}{2}\right)}, \hat{B}_{-} \hat{B}_{3} \hat{G}_{22}, \\
\hat{B}_{-} \hat{B}_{3} V^{\left(\frac{3}{2}\right)}, \hat{B}_{-} T^{(1)} \hat{G}_{22}, \hat{B}_{-} T^{(1)} V^{\left(\frac{3}{2}\right)}, \hat{B}_{-} \partial \hat{G}_{22}, \\
\hat{B}_{-} \partial V^{\left(\frac{3}{2}\right)}, \hat{A}_{3} W_{+}^{\left(\frac{5}{2}\right)}, \hat{A}_{3} \hat{A}_{3} \hat{G}_{21}, \hat{A}_{3} \hat{A}_{3} T_{+}^{\left(\frac{3}{2}\right)}, \hat{A}_{3} \hat{B}_{3} \hat{G}_{21}, \hat{A}_{3} \hat{B}_{3} T_{+}^{\left(\frac{3}{2}\right)}, \\
\hat{A}_{3} \partial \hat{G}_{21}, \hat{A}_{3} \partial T_{+}^{\left(\frac{3}{2}\right)}, \hat{B}_{3} W_{+}^{\left(\frac{5}{2}\right)}, \hat{B}_{3} \hat{B}_{3} \hat{G}_{21}, \hat{B}_{3} \hat{B}_{3} T_{+}^{\left(\frac{3}{2}\right)}, \hat{B}_{3} T^{(1)} \hat{G}_{21}, \hat{B}_{3} T^{(1)} T_{+}^{\left(\frac{3}{2}\right)}, \\
\hat{B}_{3} \partial \hat{G}_{21}, \hat{B}_{3} \partial T_{+}^{\left(\frac{3}{2}\right)}, T^{(1)} W_{+}^{\left(\frac{5}{2}\right)}, T^{(1)} \hat{A}_{-} \hat{G}_{11}, T^{(1)} \hat{A}_{-} U^{\left(\frac{3}{2}\right)}, T^{(1)} \hat{A}_{3} \hat{G}_{21}, \\
T^{(1)} \hat{A}_{3} T_{+}^{\left(\frac{3}{2}\right)}, T^{(1)} T^{(1)} \hat{G}_{21}, T^{(1)} T^{(1)} T_{+}^{\left(\frac{3}{2}\right)}, T^{(1)} \partial \hat{G}_{21}, T^{(1)} \partial T_{+}^{\left(\frac{3}{2}\right)}, \partial W_{+}^{\left(\frac{5}{2}\right)}, \partial \hat{A}_{-} \hat{G}_{11}, \\
\partial \hat{A}_{-} U^{\left(\frac{3}{2}\right)}, \partial \hat{A}_{3} \hat{G}_{21}, \partial \hat{A}_{3} T_{+}^{\left(\frac{3}{2}\right)}, \partial \hat{B}_{-} \hat{G}_{22}, \partial \hat{B}_{-} V^{\left(\frac{3}{2}\right)}, \partial \hat{B}_{3} \hat{G}_{21}, \\
\partial \hat{B}_{3} T_{+}^{\left(\frac{3}{2}\right)}, \partial T^{(1)} \hat{G}_{21}, \partial T^{(1)} T_{+}^{\left(\frac{3}{2}\right)}, \partial^{2} \hat{G}_{21}, \partial^{2} T_{+}^{\left(\frac{3}{2}\right)}, \hat{G}_{21} \hat{T}_{1} \hat{G}_{21} T^{(2)}, \hat{G}_{21} W^{(2)}, T_{+}^{\left(\frac{3}{2}\right)} \hat{T}, \\
T_{+}^{\left(\frac{3}{2}\right)} T^{(2)}, T_{+}^{\left(\frac{3}{2}\right)} W^{(2)}, \hat{G}_{11} V_{+}^{(2)}, U^{\left(\frac{3}{2}\right)} V_{+}^{(2)}, \hat{G}_{22} U_{+}^{(2)}, V^{\left(\frac{3}{2}\right)} U_{+}^{(2)}\end{array}$ \\
\hline$-\frac{(3+k)}{(5+k)}$ & $\begin{array}{c}\hat{A}_{+} \hat{A}_{-} \hat{G}_{12}, \hat{A}_{+} \hat{A}_{-} T_{-}^{\left(\frac{3}{2}\right)}, \hat{A}_{+} V^{\left(\frac{5}{2}\right)},, \hat{A}_{+} \hat{A}_{3} \hat{G}_{22}, \hat{A}_{+} \hat{A}_{3} V^{\left(\frac{3}{2}\right)}, \hat{A}_{+} \hat{B}_{+} \hat{G}_{21}, \\
\hat{A}_{+} \hat{B}_{+} T_{+}^{\left(\frac{3}{2}\right)}, \hat{A}_{+} \hat{B}_{3} \hat{G}_{22}, \hat{A}_{+} \hat{B}_{3} V^{\left(\frac{3}{2}\right)}, \hat{A}_{+} \partial \hat{G}_{22}, \hat{A}_{+} \partial V^{\left(\frac{3}{2}\right)}, \hat{B}_{-} \hat{B}_{+} \hat{G}_{12}, \hat{B}_{-} \hat{B}_{+} T_{-}^{\left(\frac{3}{2}\right)}, \\
\hat{B}_{+} U^{\left(\frac{5}{2}\right)}, \hat{B}_{+} \hat{A}_{3} \hat{G}_{11}, \hat{B}_{+} \hat{A}_{3} U^{\left(\frac{3}{2}\right)}, \hat{B}_{+} \hat{B}_{3} \hat{G}_{11}, \\
\hat{B}_{+} \hat{B}_{3} U^{\left(\frac{3}{2}\right)}, \hat{B}_{+} T^{(1)} \hat{G}_{11}, \hat{B}_{+} T^{(1)} U^{\left(\frac{3}{2}\right)}, \hat{B}_{+} \partial \hat{G}_{11}, \\
\hat{B}_{+} \partial U^{\left(\frac{3}{2}\right)}, \hat{A}_{3} W_{-}^{\left(\frac{5}{2}\right)}, \hat{A}_{3} \hat{A}_{3} \hat{G}_{12}, \hat{A}_{3} \hat{A}_{3} T_{-}^{\left(\frac{3}{2}\right)}, \hat{A}_{3} \hat{B}_{3} \hat{G}_{12}, \hat{A}_{3} \hat{B}_{3} T_{-}^{\left(\frac{3}{2}\right)}, \\
\hat{A}_{3} \partial \hat{G}_{12}, \hat{A}_{3} \partial T_{-}^{\left(\frac{3}{2}\right)}, \hat{B}_{3} W_{-}^{\left(\frac{5}{2}\right)}, \hat{B}_{3} \hat{B}_{3} \hat{G}_{12}, \hat{B}_{3} \hat{B}_{3} T_{-}^{\left(\frac{3}{2}\right)}, \hat{B}_{3} T^{(1)} \hat{G}_{12}, \hat{B}_{3} T^{(1)} T_{-}^{\left(\frac{3}{2}\right)}, \\
\hat{B}_{3} \partial \hat{G}_{12}, \hat{B}_{3} \partial T_{-}^{\left(\frac{3}{2}\right)}, T^{(1)} W_{-}^{\left(\frac{5}{2}\right)}, T^{(1)} \hat{A}_{+} \hat{G}_{22}, T^{(1)} \hat{A}_{+} V^{\left(\frac{3}{2}\right)}, T^{(1)} \hat{A}_{3} \hat{G}_{12}, \\
T^{(1)} \hat{A}_{3} T_{-}^{\left(\frac{3}{2}\right)}, T^{(1)} T^{(1)} \hat{G}_{12}, T^{(1)} T^{(1)} T_{-}^{\left(\frac{3}{2}\right)}, T^{(1)} \partial \hat{G}_{12}, T^{(1)} \partial T_{-}^{\left(\frac{3}{2}\right)}, \partial W_{-}^{\left(\frac{5}{2}\right)}, \partial \hat{A}_{+} \hat{G}_{22}, \\
\partial \hat{A}_{+} V^{\left(\frac{3}{2}\right)}, \partial \hat{A}_{3} \hat{G}_{12}, \partial \hat{A}_{3} T_{-}^{\left(\frac{3}{2}\right)}, \partial \hat{B}_{+} \hat{G}_{11}, \partial \hat{B}_{+} U^{\left(\frac{3}{2}\right)}, \partial \hat{B}_{3} \hat{G}_{12}, \\
\partial \hat{B}_{3} T_{-}^{\left(\frac{3}{2}\right)}, \partial T^{(1)} \hat{G}_{12}, \partial T^{(1)} T_{-}^{\left(\frac{3}{2}\right)}, \partial^{2} \hat{G}_{12}, \partial^{2} T_{-}^{\left(\frac{3}{2}\right)}, \hat{G}_{12} \hat{T}_{12} \hat{G}_{12} T^{(2)}, \hat{G}_{12} W^{(2)}, T_{-}^{\left(\frac{3}{2}\right)} \hat{T}, \\
T_{-}^{\left(\frac{3}{2}\right)} T^{(2)}, T_{-}^{\left(\frac{3}{2}\right)} W^{(2)}, \hat{G}_{22} U_{-}^{(2)}, V^{\left(\frac{3}{2}\right)} U_{-}^{(2)}, \hat{G}_{11} V_{-}^{(2)}, U^{\left(\frac{3}{2}\right)} V_{-}^{(2)}\end{array}$ \\
\hline
\end{tabular}

Table 6. The U(1) charges for the spin- $\frac{7}{2}$ fields. The fields appearing in the first-order pole in appendix (C.45) and (C.44) correspond to the above ones respectively. The fields in the first row are conjugated to those in the second row.

currents and 16 currents. In the right hand side of these OPEs, we only focus on the linear higher spin current terms. See also appendix $\mathrm{C}$ where one obtains all the detailed results. The first result is as follows:

$$
\begin{aligned}
& \hat{G}_{11} \times\left(\begin{array}{cccc}
T^{(1)}, & T_{+}^{\left(\frac{3}{2}\right)}, & T_{-}^{\left(\frac{3}{2}\right)}, & T^{(2)} \\
U^{\left(\frac{3}{2}\right)}, & U_{+}^{(2)}, & U_{-}^{(2)}, & U^{\left(\frac{5}{2}\right)} \\
V^{\left(\frac{3}{2}\right)}, & V_{+}^{(2)}, & V_{-}^{(2)}, & V^{\left(\frac{5}{2}\right)} \\
W^{(2)}, & W_{+}^{\left(\frac{5}{2}\right)}, & W_{-}^{\left(\frac{5}{2}\right)}, & W^{(3)}
\end{array}\right) \\
& \rightarrow\left(\begin{array}{cccc}
U^{\left(\frac{3}{2}\right)}, & U_{+}^{(2)}, & U_{-}^{(2)}, & U^{\left(\frac{3}{2}\right)}, U^{\left(\frac{5}{2}\right)} \\
0, & 0, & 0, & 0 \\
T^{(1)}, W^{(2)}, & T_{+}^{\left(\frac{3}{2}\right)}, W_{+}^{\left(\frac{5}{2}\right)}, & T_{-}^{\left(\frac{3}{2}\right)}, W_{-}^{\left(\frac{5}{2}\right)}, & T^{(1)}, T^{(2)}, W^{(2)}, W^{(3)} \\
U^{\left(\frac{3}{2}\right)}, & U_{+}^{(2)}, & U_{-}^{(2)}, & U^{\left(\frac{3}{2}\right)}, U^{\left(\frac{5}{2}\right)}
\end{array}\right) .
\end{aligned}
$$




\begin{tabular}{|c|c|}
\hline $\mathrm{U}(1)$ charge & Composite fields of spin- $\frac{7}{2}$ \\
\hline & $\begin{array}{c}\hat{A}_{-} \hat{A}_{+} \hat{G}_{11}, \hat{A}_{-} \hat{A}_{+} U^{\left(\frac{3}{2}\right)}, \hat{B}_{+} \hat{B}_{-} \hat{G}_{11}, \hat{B}_{+} \hat{B}_{-} U^{\left(\frac{3}{2}\right)}, \hat{B}_{-} \hat{A}_{+} \hat{G}_{22}, \hat{B}_{-} \hat{A}_{+} V^{\left(\frac{3}{2}\right)} \\
\hat{B}_{-} W_{-}^{\left(\frac{5}{2}\right)}, \hat{B}_{-} \hat{A}_{3} \hat{G}_{12}, \hat{B}_{-} \hat{A}_{3} T_{-}^{\left(\frac{3}{2}\right)}, \hat{B}_{-} \hat{B}_{3} \hat{G}_{12}, \hat{B}_{-} \hat{B}_{3} T_{-}^{\left(\frac{3}{2}\right)}, \hat{B}_{-} T^{(1)} \hat{G}_{12} \\
\hat{B}_{-} T^{(1)} T_{-}^{\left(\frac{3}{2}\right)}, \hat{B}_{-} \partial \hat{G}_{12}, \hat{B}_{-} \partial T_{-}^{\left(\frac{3}{2}\right)}, \hat{A}_{+} W_{+}^{\left(\frac{5}{2}\right)}, \hat{A}_{+} \hat{A}_{3} \hat{G}_{21}, \hat{A}_{+} \hat{A}_{3} T_{+}^{\left(\frac{3}{2}\right)} \\
\hat{A}_{+} \hat{B}_{3} \hat{G}_{21}, \hat{A}_{+} \hat{B}_{3} T_{+}^{\left(\frac{3}{2}\right)}, \hat{A}_{+} T^{(1)} \hat{G}_{21}, \hat{A}_{+} T^{(1)} T_{+}^{\left(\frac{3}{2}\right)}, \hat{A}_{+} \partial \hat{G}_{21}, \hat{A}_{+} \partial T_{+}^{\left(\frac{3}{2}\right)} \\
\hat{A}_{3} U^{\left(\frac{5}{2}\right)}, \hat{A}_{3} \hat{A}_{3} \hat{G}_{11}, \hat{A}_{3} \hat{A}_{3} U^{\left(\frac{3}{2}\right)}, \hat{A}_{3} \hat{B}_{3} \hat{G}_{11}, \hat{A}_{3} \hat{B}_{3} U^{\left(\frac{3}{2}\right)}, \hat{A}_{3} T^{(1)} \hat{G}_{11} \\
\hat{A}_{3} T^{(1)} U^{\left(\frac{3}{2}\right)}, \hat{A}_{3} \partial \hat{G}_{11}, \hat{A}_{3} \partial U^{\left(\frac{3}{2}\right)}, \hat{B}_{3} U^{\left(\frac{5}{2}\right)}, \hat{B}_{3} \hat{B}_{3} \hat{G}_{11}, \hat{B}_{3} \hat{B}_{3} U^{\left(\frac{3}{2}\right)}, \hat{B}_{3} T^{(1)} \hat{G}_{11} \\
\hat{B}_{3} T^{(1)} U^{\left(\frac{3}{2}\right)}, \hat{B}_{3} \partial \hat{G}_{11}, \hat{B}_{3} \partial U^{\left(\frac{3}{2}\right)}, T^{(1)} U^{\left(\frac{5}{2}\right)}, T^{(1)} T^{(1)} \hat{G}_{11}, \\
T^{(1)} T^{(1)} U^{\left(\frac{3}{2}\right)}, T^{(1)} \partial \hat{G}_{11}, T^{(1)} \partial U^{\left(\frac{3}{2}\right)}, \partial \hat{A}_{+} \hat{G}_{21}, \partial \hat{A}_{+} T_{+}^{\left(\frac{3}{2}\right)} \\
\partial \hat{B}_{-} \hat{G}_{12}, \partial \hat{B}_{-} T_{-}^{\left(\frac{3}{2}\right)}, \partial \hat{B}_{3} \hat{G}_{11}, \partial \hat{B}_{3} U^{\left(\frac{3}{2}\right)}, \partial T^{(1)} \hat{G}_{11}, \partial T^{(1)} U^{\left(\frac{3}{2}\right)} \\
\partial^{2} \hat{G}_{11}, \partial^{2} U^{\left(\frac{3}{2}\right)}, \partial U^{\left(\frac{5}{2}\right)}, \hat{G}_{21} U_{-}^{(2)}, T_{+}^{\left(\frac{3}{2}\right)} U_{-}^{(2)}, \hat{G}_{12} U_{+}^{(2)}, T_{-}^{\left(\frac{3}{2}\right)} U_{+}^{(2)}, \hat{G}_{11} \hat{T}_{11} \hat{G}_{11} T^{(2)} \\
\hat{G}_{11} W^{(2)}, \hat{G}_{11} \partial \hat{A}_{3}, U^{\left(\frac{3}{2}\right)} \hat{T}, U^{\left(\frac{3}{2}\right)} T^{(2)}, U^{\left(\frac{3}{2}\right)} W^{(2)}, U^{\left(\frac{3}{2}\right)} \partial \hat{A}_{3}\end{array}$ \\
\hline$-\frac{(-3+k)}{(5+k)}$ & $\begin{array}{c}\hat{A}_{+} \hat{A}_{-} \hat{G}_{22}, \hat{A}_{+} \hat{A}_{-} V^{\left(\frac{3}{2}\right)}, \hat{B}_{-} \hat{B}_{+} \hat{G}_{22}, \hat{B}_{-} \hat{B}_{+} V^{\left(\frac{3}{2}\right)}, \hat{B}_{+} \hat{A}_{-} \hat{G}_{11}, \hat{B}_{+} \hat{A}_{-} U^{\left(\frac{3}{2}\right)}, \\
\hat{B}_{+} W_{+}^{\left(\frac{5}{2}\right)}, \hat{B}_{+} \hat{A}_{3} \hat{G}_{21}, \hat{B}_{+} \hat{A}_{3} T_{+}^{\left(\frac{3}{2}\right)}, \hat{B}_{+} \hat{B}_{3} \hat{G}_{21}, \hat{B}_{+} \hat{B}_{3} T_{+}^{\left(\frac{3}{2}\right)}, \hat{B}_{+} T^{(1)} \hat{G}_{21}, \\
\hat{B}_{+} T^{(1)} T_{+}^{\left(\frac{3}{2}\right)}, \hat{B}_{+} \partial \hat{G}_{21}, \hat{B}_{+} \partial T_{+}^{\left(\frac{3}{2}\right)}, \hat{A}_{-} W_{-}^{\left(\frac{5}{2}\right)}, \hat{A}_{-} \hat{A}_{3} \hat{G}_{12}, \hat{A}_{-} \hat{A}_{3} T_{-}^{\left(\frac{3}{2}\right)} \\
\hat{A}_{-} \hat{B}_{3} \hat{G}_{12}, \hat{A}_{-} \hat{B}_{3} T_{-}^{\left(\frac{3}{2}\right)}, \hat{A}_{-} T^{(1)} \hat{G}_{12}, \hat{A}_{-} T^{(1)} T_{-}^{\left(\frac{3}{2}\right)} \\
\hat{A}_{-} \partial \hat{G}_{12}, \hat{A}_{-} \partial T_{-}^{\left(\frac{3}{2}\right)}, \hat{A}_{3} V^{\left(\frac{5}{2}\right)}, \hat{A}_{3} \hat{A}_{3} \hat{G}_{22}, \hat{A}_{3} \hat{A}_{3} V^{\left(\frac{3}{2}\right)}, \hat{A}_{3} \hat{B}_{3} \hat{G}_{22}, \\
\hat{A}_{3} \hat{B}_{3} V^{\left(\frac{3}{2}\right)}, \hat{A}_{3} T^{(1)} \hat{G}_{22}, \hat{A}_{3} T^{(1)} V^{\left(\frac{3}{2}\right)}, \hat{A}_{3} \partial \hat{G}_{22}, \hat{A}_{3} \partial V^{\left(\frac{3}{2}\right)}, \hat{B}_{3} V^{\left(\frac{5}{2}\right)}, \hat{B}_{3} \hat{B}_{3} \hat{G}_{22}, \\
\hat{B}_{3} \hat{B}_{3} V^{\left(\frac{3}{2}\right)}, \hat{B}_{3} T^{(1)} \hat{G}_{22}, \hat{B}_{3} T^{(1)} V^{\left(\frac{3}{2}\right)}, \hat{B}_{3} \partial \hat{G}_{22}, \hat{B}_{3} \partial V^{\left(\frac{3}{2}\right)}, T^{(1)} V^{\left(\frac{5}{2}\right)}, T^{(1)} T^{(1)} \hat{G}_{22}, \\
T^{(1)} T^{(1)} V^{\left(\frac{3}{2}\right)}, T^{(1)} \partial \hat{G}_{22}, T^{(1)} \partial V^{\left(\frac{3}{2}\right)}, \partial \hat{A}_{-} \hat{G}_{12}, \partial \hat{A}_{-} T_{-}^{\left(\frac{3}{2}\right)}, \\
\partial \hat{B}_{+} \hat{G}_{21}, \partial \hat{B}_{+} T_{+}^{\left(\frac{3}{2}\right)}, \partial \hat{B}_{3} \hat{G}_{22}, \partial \hat{B}_{3} V^{\left(\frac{3}{2}\right)}, \partial T^{(1)} \hat{G}_{22}, \partial T^{(1)} V^{\left(\frac{3}{2}\right)}, \\
\partial^{2} \hat{G}_{22}, \partial^{2} V^{\left(\frac{3}{2}\right)}, \partial V^{\left(\frac{5}{2}\right)}, \hat{G}_{12} V_{+}^{(2)}, T_{-}^{\left(\frac{3}{2}\right)} V_{+}^{(2)}, \hat{G}_{21} V_{-}^{(2)}, T_{+}^{\left(\frac{3}{2}\right)} V_{-}^{(2)}, \hat{G}_{22} \hat{T}_{2}, \hat{G}_{22} T^{(2)}, \\
\hat{G}_{22} W^{(2)}, \hat{G}_{22} \partial \hat{A}_{3}, V^{\left(\frac{3}{2}\right)} \hat{T}, V^{\left(\frac{3}{2}\right)} T^{(2)}, V^{\left(\frac{3}{2}\right)} W^{(2)}, V^{\left(\frac{3}{2}\right)} \partial \hat{A}_{3}\end{array}$ \\
\hline
\end{tabular}

Table 7. The $\mathrm{U}(1)$ charges for the spin- $-\frac{7}{2}$ fields. The fields appearing in the first-order pole in appendix (C.43) and (C.46) correspond to the above ones respectively. The fields in two rows are conjugated to each other.

There are no higher spin currents, denoted by zeros in (6.1) corresponding to the row containing $G_{11}(w)$ when one writes (2.44) in terms of $4 \times 4$ matrix, in the OPEs between the current $\hat{G}_{11}(z)$ and the four currents living in the second multiplet of (4.1). These four currents appear in first and last rows in the final expression of (6.1). Also the current $U^{\left(\frac{3}{2}\right)}(w)$ appears in the last columns of these rows by conformal dimensional analysis. Finally, the first and last multiplets of (4.1) appear in the third row in (6.1). Of course, the currents $T^{(1)}(w)$ and $W^{(2)}(w)$ can appear in the last column of this row. 
Similarly, it turns out that the following result for the spin- $\frac{3}{2}$ current and 16 currents is given by

$$
\begin{aligned}
\hat{G}_{12} & \times\left(\begin{array}{cccc}
T^{(1)}, & T_{+}^{\left(\frac{3}{2}\right)}, & T_{-}^{\left(\frac{3}{2}\right)}, & T^{(2)} \\
U^{\left(\frac{3}{2}\right)}, & U_{+}^{(2)}, & U_{-}^{(2)}, & U^{\left(\frac{5}{2}\right)} \\
V^{\left(\frac{3}{2}\right)}, & V_{+}^{(2)}, & V_{-}^{(2)}, & V^{\left(\frac{5}{2}\right)} \\
W^{(2)}, & W_{+}^{\left(\frac{5}{2}\right)}, & W_{-}^{\left(\frac{5}{2}\right)}, & W^{(3)}
\end{array}\right) \\
& \rightarrow\left(\begin{array}{cccc}
T_{-}^{\left(\frac{3}{2}\right)}, & T^{(1)}, T^{(2)}, & 0, & T_{-}^{\left(\frac{3}{2}\right)} \\
U_{-}^{(2)}, & U^{\left(\frac{3}{2}\right)}, U^{\left(\frac{5}{2}\right)}, & 0, & U_{-}^{(2)} \\
V_{-}^{(2)}, & V^{\left(\frac{3}{2}\right)}, V^{\left(\frac{5}{2}\right)}, & 0, & V_{-}^{(2)} \\
T_{-}^{\left(\frac{3}{2}\right)}, W_{-}^{\left(\frac{5}{2}\right)}, T^{(1)}, T^{(2)}, W^{(2)}, W^{(3)}, & 0, T_{-}^{\left(\frac{3}{2}\right)}, W_{-}^{\left(\frac{5}{2}\right)}
\end{array}\right) .
\end{aligned}
$$

There are no higher spin currents, denoted by zeros in (6.2) corresponding to the column containing $G_{12}(w)$ in $(2.44)$, in the OPEs between the current $\hat{G}_{12}(z)$ and the four currents living in the third component of each multiplet of (4.1). These four currents appear in the first and the last columns in the final expression of (6.2). Also the current $T_{-}^{\left(\frac{3}{2}\right)}(w)$ appears in the last rows of these columns. Finally, the first and last components in each multiplet of (4.1) appear in the second column in (6.2). Of course, the currents $T^{(1)}(w)$ and $W^{(2)}(w)$ can appear in the last row of this column.

One has the following result

$$
\begin{aligned}
\hat{G}_{21} & \times\left(\begin{array}{cccc}
T^{(1)}, & T_{+}^{\left(\frac{3}{2}\right)}, & T_{-}^{\left(\frac{3}{2}\right)}, & T^{(2)} \\
U^{\left(\frac{3}{2}\right)}, & U_{+}^{(2)}, & U_{-}^{(2)}, & U^{\left(\frac{5}{2}\right)} \\
V^{\left(\frac{3}{2}\right)}, & V_{+}^{(2)}, & V_{-}^{(2)}, & V^{\left(\frac{5}{2}\right)} \\
W^{(2)}, & W_{+}^{\left(\frac{5}{2}\right)}, & W_{-}^{\left(\frac{5}{2}\right)}, & W^{(3)}
\end{array}\right) \\
& \rightarrow\left(\begin{array}{cccc}
T_{+}^{\left(\frac{3}{2}\right)}, & 0, & T^{(1)}, T^{(2)}, & T_{+}^{\left(\frac{3}{2}\right)} \\
U_{+}^{(2)}, & 0, & U^{\left(\frac{3}{2}\right)}, U^{\left(\frac{5}{2}\right)}, & U_{+}^{(2)} \\
V_{+}^{(2)}, & 0, & V^{\left(\frac{3}{2}\right)}, V^{\left(\frac{5}{2}\right)}, & V_{+}^{(2)} \\
T_{+}^{\left(\frac{3}{2}\right)}, W_{+}^{\left(\frac{5}{2}\right)}, & 0, & T^{(1)}, T^{(2)}, W^{(2)}, W^{(3)}, T_{+}^{\left(\frac{3}{2}\right)}, W_{+}^{\left(\frac{5}{2}\right)}
\end{array}\right) .
\end{aligned}
$$

There are no higher spin currents, denoted by zeros in (6.3) corresponding to the column containing $G_{21}(w)$ in (2.44), in the OPEs between the current $\hat{G}_{21}(z)$ and the four currents living in the second component of each multiplet of (4.1). These four currents appear in the first and the last columns in the final expression of (6.3). Also the current $T_{+}^{\left(\frac{3}{2}\right)}(w)$ appears in the last rows of these columns. Finally, the first and last components in each multiplet of (4.1) appear in the third column in (6.3). Of course, the currents $T^{(1)}(w)$ and $W^{(2)}(w)$ can appear in the last row of this column. 
Finally, one obtains the following result

$$
\begin{aligned}
& \hat{G}_{22} \times\left(\begin{array}{cccc}
T^{(1)}, & T_{+}^{\left(\frac{3}{2}\right)}, & T_{-}^{\left(\frac{3}{2}\right)}, & T^{(2)} \\
U^{\left(\frac{3}{2}\right)}, & U_{+}^{(2)}, & U_{-}^{(2)}, & U^{\left(\frac{5}{2}\right)} \\
V^{\left(\frac{3}{2}\right)}, & V_{+}^{(2)}, & V_{-}^{(2)}, & V^{\left(\frac{5}{2}\right)} \\
W^{(2)}, & W_{+}^{\left(\frac{5}{2}\right)}, & W_{-}^{\left(\frac{5}{2}\right)}, & W^{(3)}
\end{array}\right) \\
& \rightarrow\left(\begin{array}{cccc}
V^{\left(\frac{3}{2}\right)}, & V_{+}^{(2)}, & V_{-}^{(2)}, & V^{\left(\frac{3}{2}\right)}, V^{\left(\frac{5}{2}\right)} \\
T^{(1)}, W^{(2)}, T_{+}^{\left(\frac{3}{2}\right)}, W_{+}^{\left(\frac{5}{2}\right)}, & T_{-}^{\left(\frac{3}{2}\right)}, W_{-}^{\left(\frac{5}{2}\right)}, & T^{(1)}, T^{(2)}, W^{(2)}, W^{(3)} \\
0, & 0, & 0, & 0 \\
V^{\left(\frac{3}{2}\right)}, & V_{+}^{(2)}, & V_{-}^{(2)}, & V^{\left(\frac{3}{2}\right)}, V^{\left(\frac{5}{2}\right)}
\end{array}\right) .
\end{aligned}
$$

There are no higher spin currents, denoted by zeros in (6.4) corresponding to the row containing $G_{22}(w)$ in (2.44), in the OPEs between the current $\hat{G}_{22}(z)$ and the four currents living in the third multiplet of (4.1). These four currents appear in first and last rows in the final expression of (6.4). Also the current $V^{\left(\frac{3}{2}\right)}(w)$ appears in the last columns of these rows. Finally, the first and last multiplets of (4.1) appear in the second row in (6.1). Of course, the currents $T^{(1)}(w)$ and $W^{(2)}(w)$ can appear in the last column of this row.

We present some future interesting research directions.

- The OPEs between the higher spin currents themselves.

Because the 16 higher spin currents are found explicitly, it is natural to calculate the OPEs between them [48]. The next higher spin currents are specified by the following spin contents by adding one more spin to the spin contents in (1.2):

$$
\left(2, \frac{5}{2}, \frac{5}{2}, 3\right), \quad\left(\frac{5}{2}, 3,3, \frac{7}{2}\right), \quad\left(\frac{5}{2}, 3,3, \frac{7}{2}\right), \quad\left(3, \frac{7}{2}, \frac{7}{2}, 4\right) .
$$

One expects that some of the new higher spin currents in (6.5) should arise in the OPEs between the higher spin currents in (1.2). For $N=3$, there exist finite higher spin currents consisting of (1.2) and (6.5) besides the higher spin currents in the short representation [1]. One expects that the most complicated OPE is given by the OPE between the spin-3 current and itself. It would be interesting to study the complete structure of these higher spin currents. Eventually, it is an open problem, after the work of [48] is done, to obtain the two dimensional boundary conformal field theory (in the context of higher spin theory) dual to the string theory in $A d S_{3} \times \mathbf{S}^{3} \times \mathbf{S}^{3} \times \mathbf{S}^{1}$ compactification [56-59], as suggested in [1]. See also [60] in the context of alternating spin chain $[61,62]$.

- For general $N$, how the extended currents arise?

Eventually one should have the higher spin currents for general $N$. In doing this, the first step is to write down the 16 currents in the large $\mathcal{N}=4$ linear superconformal algebra or 11 currents in the large $\mathcal{N}=4$ nonlinear superconformal algebra for general $N$. Some of the information are given in $[15,24]$. One expects that the calculations 
of OPEs for general $N$, by hand, are based on the component OPEs along the line of [63-67]. Then one should know the various identities between the products of $f$ symbols in the complex basis. One can extract some tensor structures from the OPE results in appendices $\mathrm{A}, \mathrm{B}$ and $\mathrm{C}$ because the fermionic currents transform as $(\mathbf{2}, \mathbf{2})$ under the two $\mathrm{SU}(2)$ 's from the observation of [1]. Of course, it is quite nontrivial and complicated to obtain the full algebra like as appendices A, B and C but at least one should find the currents themselves (not the whole algebra).

- Inserting the four spin- $\frac{1}{2}$ and spin-1 currents

What happens when we substitute $\hat{T}(z), \hat{A}_{1}(z),\left[\hat{A}_{2}(z)\right.$ and $\left.\hat{A}_{3}(z)\right],\left[\hat{B}_{1}(z), \hat{B}_{2}(z)\right.$ and $\left.\hat{B}_{3}(z)\right]$, and $\left[\hat{G}_{11}(z), \hat{G}_{12}(z), \hat{G}_{21}(z)\right.$ and $\left.\hat{G}_{22}(z)\right]$ using the equation (3.2), (3.5), (3.6), (3.10) and (3.13) in the large $\mathcal{N}=4$ nonlinear superconformal algebra? Then one expects that one obtains the large $\mathcal{N}=4$ linear superconformal algebra. See also the work of [24]. It would be interesting to study the other OPEs by reintroducing the four spin- $\frac{1}{2}$ currents and a spin-1 current.

- Other group realization.

In the classification of $\mathcal{N}=4$ coset theory in [15], there exists a coset model

$$
\text { Wolf } \times \mathrm{SU}(2) \times \mathrm{U}(1)=\frac{\mathrm{SO}(N+4)}{\mathrm{SO}(N) \times \mathrm{SU}(2)} \times \mathrm{U}(1) .
$$

As described in [55], the central charge for this coset model is given by $c=\frac{6(k+1)(N+1)}{(k+N+2)}$ which is equal to the one in (2.2). The first nontrivial case is for $N=4$. At least, the large $\mathcal{N}=4$ (non)linear superconformal algebra should exist. But the spin contents for the higher spin currents will be different from those in the coset model (2.1) in this paper. Therefore, it would be interesting to study the higher spin currents in the above Wolf space for (fixed) general $N$. The corresponding $\mathcal{N}=2$ current algebra in $\mathcal{N}=2$ superspace for the supersymmetric WZW model, with level $k$, on a group $G=\mathrm{SO}(N+4)$ of even dimension (for the odd dimension one can introduce the extra $\mathrm{U}(1)$ in the both numerator and the denominator of the coset), can be obtained from [35]. In general, the component expression of $\mathcal{N}=2$ current algebra in the above coset model (6.6) looks different. The previous works [68-70] in this direction will be helpful. Note that the similar coset $\frac{\mathrm{SO}(N+4)}{\mathrm{SO}(N+2) \times \mathrm{SO}(2)}$ appears in the Kazama-Suzuki model. In this case, one can also construct the higher spin currents of even spins as well.

- The general Kazama-Suzuki model and nonabelian generalization

As observed in [1], the Wolf space can be generalized to the Kazama-Suzuki coset model in (2.4)

$$
\frac{\mathrm{SU}(N+M)}{\mathrm{SU}(N) \times \mathrm{SU}(M) \times \mathrm{U}(1)}
$$


In this coset model, the central charge $c=\frac{3 k M N}{(M+N+k)}$ in (2.5) depends on $k, M$ and $N$. Then in the stringy limit where the three quantities $k, M$ and $N$ go to infinity simultaneously, the central charge behaves $N^{2}$ which is appropriate for a stringy model. It would be interesting to study the higher spin currents in this generalized coset model. For general $M$ and $N$, this coset model has at least $\mathcal{N}=2$ supersymmetry. Note that the coset (6.7) for $M=2$ case is the coset model considered in this paper. The immediate question is how to construct the $M^{2}$ fermions which can be decoupled from the algebra.

- Extension of $\mathcal{N}=2 \mathrm{U}(3)$ or $\mathrm{U}(2 \mid 1)$ Knizhnik-Bershadsky algebras and others.

In [71], the two $\mathcal{N}=2$ superconformal algebras were found. Decoupling of four spin- $\frac{1}{2}$ currents appropriately, the $\mathrm{U}(3)[43,44]$ or U(2|1) [72] Knizhnik-Bershadsky algebras were reproduced. Furthermore, the $\mathcal{N}=2$ WZW affine current superalgebra has been found. Then it is an open problem to obtain the extension of unitary KnizhnikBershadsky algebras in the higher spin current context.

For $\mathcal{N}>4$, there exists a coset construction. For example, in [73], the $\mathcal{N}=7$ and $\mathcal{N}=8$ nonlinear superconformal algebras were studied. See also [74-76]. It would be interesting to see whether these algebras can be extended or not in the context of minimal model holography even though the cosets of these models do not have any group parameters like as the rank of the group.

It would be interesting to see whether one can express the $(11+16)$ currents in terms of $\mathcal{N}=2$ superspace as done in [35] or even further $\mathcal{N}=4$ superspace in [10]. For the 16 currents of the large $\mathcal{N}=4$ linear superconformal algebra in [6], the explicit field redefinitions in [6] provide that these 16 currents are transformed into those currents in $O(4)$ extended superconformal algebra given in [10]. Then the 16 currents presented in section 2 can be combined into one single $\mathcal{N}=4$ superfield in [10]. It is not obvious how one can express the 11 currents of large $\mathcal{N}=4$ nonlinear superconformal algebra in terms of a single $\mathcal{N}=4$ superfield after decoupling one spin- 1 current and four spin- $\frac{1}{2}$ currents.

We clarify the difference between the 16 higher spin currents in this paper and the ones in [1]. One can find the particular basis where the 16 higher spin currents transform under the two $\mathrm{SU}(2)$ currents characterized by $\hat{A}_{i}(z)$ and $\hat{B}_{i}(z)$ in very simple form. ${ }^{55}$ For example, one redefines the four spin- $\frac{3}{2}$ currents appearing in (4.20), (4.13), (4.9) and (4.34) by absorbing the spin- $\frac{3}{2}$ currents $\hat{G}_{a}(w)$ of large $\mathcal{N}=4$ nonlinear algebra. Then in this new basis, there are no spin- $\frac{3}{2}$ current $\hat{G}_{a}(w)$ dependences in the right hand side of appendix (B.2) and (B.6). Furthermore, the OPEs between $\hat{G}_{a}(z)$ and these redefined (primary) spin- $\frac{3}{2}$ currents transforming as $(\mathbf{2}, \mathbf{2})$ under the $\mathrm{SU}(2) \times \mathrm{SU}(2)$ do not contain the third-order singular terms.

For the spin- 2 currents in this new basis, one can write each (redefined) hatted spin2 current in terms of each unhatted spin-2 current found in (4.1) with coefficient 1 and other composite spin-2 fields with undetermined coefficients in table 3 . Then the relative coefficients can be determined by the vanishing of second-order pole in the OPEs between

\footnotetext{
${ }^{55}$ We would like to thank the referee for raising this issue.
} 
the six spin-1 currents, $\hat{A}_{i}(z)$ and $\hat{B}_{i}(z)$, and each hatted spin-2 current, $\hat{T}^{(2)}(w), \hat{U}_{ \pm}^{(2)}(w)$, $\hat{V}_{ \pm}^{(2)}(w)$ and $\hat{W}^{(2)}(w)$. These hatted spin-2 currents should be again primary under the stress energy tensor $\hat{T}(z)$ and be $(\mathbf{3}, \mathbf{1})$ and $(\mathbf{1}, \mathbf{3})$ under the $\mathrm{SU}(2) \times \mathrm{SU}(2)$.

For the spin- $\frac{5}{2}$ currents, as before, each hatted spin- $\frac{5}{2}$ current (should be $(\mathbf{2}, \mathbf{2})$ under the $\mathrm{SU}(2) \times \mathrm{SU}(2))$ can be written as each unhatted one in (4.1) and other spin- $\frac{5}{2}$ composite fields with undetermined coefficients appearing in table 4. By requiring that the OPEs between $\hat{G}_{a}(z)$ and these redefined spin- $\frac{5}{2}$ currents do not contain the third-order singular terms and the OPEs between the above six spin- 1 currents and those hatted spin- $\frac{5}{2}$ currents do not contain the second-order singular terms (and also they should be primary under the stress energy tensor $\hat{T}(z)$ ), most of the relative coefficients are fixed. The remaining ones are determined by the condition that the OPEs between the spin-1 current $T^{(1)}(z)$ and these hatted spin- $-\frac{5}{2}$ currents do not contain the second-order singular terms [48].

For the spin-3 current, the hatted spin-3 current can be written as the unhatted one in (4.1) and other spin-3 composite fields with undetermined coefficients appearing in table 5. By requiring that the OPEs between $\hat{A}_{i}(z)$ (and $\hat{B}_{i}(z)$ ) and the hatted spin-3 current do not contain any singular terms, the relative coefficients can be fixed.

The higher spin-1 current remains unchanged because it commutes with the above six spin-1 currents in $\mathrm{SU}(2) \times \mathrm{SU}(2)$ (i.e., The OPEs do not contain any singular terms) and both the spin- 1 and the spin-3 currents, $\hat{T}^{(1)}(z)$ and $\hat{W}^{(3)}(z)$, play the role of $(\mathbf{1}, \mathbf{1})$ in $\mathrm{SU}(2) \times \mathrm{SU}(2)$ respectively as in [1].

Now we present the final 16 higher spin currents in this new basis explicitly as follows:

$$
\begin{aligned}
\hat{T}^{(1)}(z)= & T^{(1)}(z), \\
\hat{T}_{ \pm}^{\left(\frac{3}{2}\right)}(z)= & T_{ \pm}^{\left(\frac{3}{2}\right)}(z) \pm \frac{1}{2}\left(\begin{array}{c}
\hat{G}_{21} \\
\hat{G}_{12}
\end{array}\right)(z), \quad\left(\begin{array}{c}
\hat{U}^{\left(\frac{3}{2}\right)} \\
\hat{V}^{\left(\frac{3}{2}\right)}
\end{array}\right)(z)=\left(\begin{array}{c}
U^{\left(\frac{3}{2}\right)} \\
V^{\left(\frac{3}{2}\right)}
\end{array}\right)(z) \pm \frac{1}{2}\left(\begin{array}{c}
\hat{G}_{11} \\
\hat{G}_{22}
\end{array}\right)(z), \\
\hat{T}^{(2)}(z)= & T^{(2)}(z)+\frac{i}{k} T^{(1)} \hat{A}_{3}(z)+\frac{i}{3} T^{(1)} \hat{B}_{3}(z)-\frac{3(3+k)(13+9 k)}{20(2+k)(3+7 k)} T^{(1)} T^{(1)}(z) \\
& +\frac{1}{(5+k)}\left[-\frac{3 i\left(-3+3 k+2 k^{2}\right)}{(2+k)(3+7 k)} \partial \hat{A}_{3}+\frac{i(-27+k) k}{5(3+7 k)} \partial \hat{B}_{3}\right. \\
& -\frac{3\left(-3+3 k+2 k^{2}\right)}{(2+k)(3+7 k)} \hat{A}_{+} \hat{A}_{-}+\frac{k(-27+k)}{5(3+7 k)} \hat{B}_{3} \hat{B}_{3} \\
& \left.-\frac{3\left(-3+3 k+2 k^{2}\right)}{(2+k)(3+7 k)} \hat{A}_{3} \hat{A}_{3}+2 \hat{A}_{3} \hat{B}_{3}+\frac{k(-27+k)}{5(3+7 k)} \hat{B}_{+} \hat{B}_{-}\right](z), \\
\left(\begin{array}{c}
\hat{U}_{+}^{(2)} \\
\hat{V}_{-}^{(2)}
\end{array}\right)(z)= & \left(\begin{array}{l}
U_{+}^{(2)} \\
V_{-}^{(2)}
\end{array}\right)(z) \mp \frac{i}{3} T^{(1)} \hat{B}_{\mp}(z) \pm \frac{2}{(5+k)} \hat{A}_{3} \hat{B}_{\mp}(z), \\
\left(\begin{array}{c}
\hat{U}_{-}^{(2)} \\
\hat{V}_{+}^{(2)}
\end{array}\right)(z)= & \left(\begin{array}{l}
U_{-}^{(2)} \\
V_{+}^{(2)}
\end{array}\right)(z) \pm \frac{i}{k} T^{(1)} \hat{A}_{ \pm}(z) \pm \frac{2}{(5+k)} \hat{A}_{ \pm} \hat{B}_{3}(z), \\
\hat{W}^{(2)}(z)= & W^{(2)}(z)-\frac{i}{k} T^{(1)} \hat{A}_{3}(z)+\frac{i}{3} T^{(1)} \hat{B}_{3}(z)-\frac{3(13+9 k)}{20(2+k)} T^{(1)} T^{(1)}(z) \\
& +\frac{1}{(5+k)}\left[\frac{3 i}{(2+k)} \partial \hat{A}_{3}+\frac{i k}{5} \partial \hat{B}_{3}+\frac{3}{(2+k)} \hat{A}_{+} \hat{A}_{-}\right. \\
& \left.+\frac{3}{(2+k)} \hat{A}_{3} \hat{A}_{3}-2 \hat{A}_{3} \hat{B}_{3}+\frac{k}{5} \hat{B}_{+} \hat{B}_{-}+\frac{k}{5} \hat{B}_{3} \hat{B}_{3}\right](z),
\end{aligned}
$$




$$
\begin{aligned}
& \hat{U}^{\left(\frac{5}{2}\right)}(z)=U^{\left(\frac{5}{2}\right)}(z)+\frac{1}{(5+k)\left(-25+41 k+38 k^{2}\right)}\left[16 i k(8+k) \hat{A}_{+} \hat{G}_{21}\right. \\
& +2 i\left(-25+169 k+54 k^{2}\right) \hat{A}_{+} T_{+}^{\left(\frac{3}{2}\right)} \\
& +2 i(5+2 k)(-5+23 k) \hat{A}_{3} \hat{G}_{11}+4 i(5+2 k)(-5+23 k) \hat{A}_{3} U^{\left(\frac{3}{2}\right)} \\
& -6 i(-1+k)(1+k)(5+2 k) \hat{B}_{-} \hat{G}_{12}+12 i(-1+k)(1+k)(5+2 k) \hat{B}_{-} T_{-}^{\left(\frac{3}{2}\right)} \\
& +12 i(-1+k)(1+k)(5+2 k) \hat{B}_{3} U^{\left(\frac{3}{2}\right)}+(-3+k)(17+10 k)(5+k) T^{(1)} \hat{G}_{11} \\
& +2 i(8+k)\left(-5+5 k+6 k^{2}\right) \hat{B}_{3} \hat{G}_{11}+\frac{2}{3}(-3+k)\left(50+71 k+14 k^{2}\right) \partial \hat{G}_{11} \\
& \left.+\frac{4}{3}\left(-125-204 k-9 k^{2}+14 k^{3}\right) \partial U^{\left(\frac{3}{2}\right)}\right](z), \\
& \hat{V}^{\left(\frac{5}{2}\right)}(z)=V^{\left(\frac{5}{2}\right)}(z)+\frac{1}{(5+k)\left(-25+41 k+38 k^{2}\right)}\left[-16 i k(8+k) \hat{A}_{-} \hat{G}_{12}\right. \\
& +32 i k(8+k) \hat{A}_{-} T_{-}^{\left(\frac{3}{2}\right)}-2 i(5+2 k)(-5+23 k) \hat{A}_{3} \hat{G}_{22}+32 i k(8+k) \hat{A}_{3} V^{\left(\frac{3}{2}\right)} \\
& +2 i(8+k)\left(-5+5 k+6 k^{2}\right) \hat{B}_{+} \hat{G}_{21}+2 i\left(-11+8 k+12 k^{2}\right)(5+k) \hat{B}_{+} T_{+}^{\left(\frac{3}{2}\right)} \\
& +4 i(8+k)\left(-5+5 k+6 k^{2}\right) \hat{B}_{3} V^{\left(\frac{3}{2}\right)}-(-3+k)(17+10 k)(5+k) T^{(1)} \hat{G}_{22} \\
& -2 i(8+k)\left(-5+5 k+6 k^{2}\right) \hat{B}_{3} \hat{G}_{22}+\frac{2}{3}(-3+k)\left(50+71 k+14 k^{2}\right) \partial \hat{G}_{22} \\
& \left.-\frac{4}{3}(1+k)\left(-175+53 k+14 k^{2}\right) \partial V^{\left(\frac{3}{2}\right)}\right](z), \\
& \hat{W}_{ \pm}^{\left(\frac{5}{2}\right)}(z)=W_{ \pm}^{\left(\frac{5}{2}\right)}(z)+\frac{1}{(5+k)\left(-25+41 k+38 k^{2}\right)}\left[ \pm 16 i k(8+k) \hat{A}_{\mp}\left(\begin{array}{c}
\hat{G}_{11} \\
\hat{G}_{22}
\end{array}\right)\right. \\
& +2 i\left(-25+169 k+54 k^{2}\right) \hat{A}_{\mp}\left(\begin{array}{l}
U^{\left(\frac{3}{2}\right)} \\
V^{\left(\frac{3}{2}\right)}
\end{array}\right) \\
& \pm 2 i\left(-25-23 k+30 k^{2}\right) \hat{A}_{3}\left(\begin{array}{c}
\hat{G}_{21} \\
\hat{G}_{12}
\end{array}\right)-32 i k(8+k) \hat{A}_{3} T_{ \pm}^{\left(\frac{3}{2}\right)} \\
& \pm 6 i(-1+k)(1+k)(5+2 k) \hat{B}_{\mp}\left(\begin{array}{c}
\hat{G}_{22} \\
\hat{G}_{11}
\end{array}\right) \\
& -2 i\left(-11+8 k+12 k^{2}\right)(5+k) \hat{B}_{\mp}\left(\begin{array}{c}
V^{\left(\frac{3}{2}\right)} \\
U^{\left(\frac{3}{2}\right)}
\end{array}\right) \\
& \pm 2 i(8+k)\left(-5+5 k+6 k^{2}\right) \hat{B}_{3}\left(\begin{array}{c}
\hat{G}_{21} \\
\hat{G}_{12}
\end{array}\right)+12 i(-1+k)(1+k)(5+2 k) \hat{B}_{3} T_{ \pm}^{\left(\frac{3}{2}\right)} \\
& \pm(-3+k)(17+10 k)(5+k) T^{(1)}\left(\begin{array}{c}
\hat{G}_{21} \\
\hat{G}_{12}
\end{array}\right) \\
& +\frac{2}{3}(8+k)\left(-25-7 k+14 k^{2}\right) \partial\left(\begin{array}{c}
\hat{G}_{21} \\
\hat{G}_{12}
\end{array}\right) \\
& \left. \pm \frac{4}{3}(-3+k)\left(50+71 k+14 k^{2}\right) \partial T_{ \pm}^{\left(\frac{3}{2}\right)}\right](z),
\end{aligned}
$$




$$
\begin{aligned}
& \hat{W}^{(3)}(z)=W^{(3)}(z)+\frac{1}{(k+5)}\left[\frac{4}{(k+5)} \hat{A}_{+} \partial \hat{A}_{-}-\frac{6}{(k+5)} \hat{A}_{-} \partial \hat{A}_{+}+\frac{6}{(5+k)} \hat{A}_{3} \partial \hat{A}_{3}\right. \\
& -i \hat{A}_{3} \partial T^{(1)}-\partial \hat{T}-\partial W^{(2)}+i \hat{B}_{3} \partial T^{(1)}-\frac{8 i}{(k+5)} \hat{A}_{+} \hat{A}_{-} \hat{A}_{3}-T^{(1)} \hat{A}_{3} \hat{A}_{3} \\
& \left.-T^{(1)} \hat{A}_{+} \hat{A}_{-}-\frac{8 i}{(k+5)} \hat{A}_{3} \hat{A}_{3} \hat{A}_{3}-\frac{8 i}{(k+5)} \hat{A}_{3} \hat{B}_{3} \hat{B}_{3}\right](z) \\
& +\frac{1}{\left(786 k^{3}+3727 k^{2}+2920 k-1925\right)(k+5)} \\
& \times\left[-\frac{3\left(7860 k^{5}+100436 k^{4}+512137 k^{3}+950183 k^{2}+359535 k-296975\right)}{2(23 k+19)} T^{(1)} \hat{T}\right. \\
& -\frac{i}{2}\left(534 k^{4}+6689 k^{3}+23191 k^{2}+16723 k-11065\right) T^{(1)} \partial \hat{B}_{3} \\
& +\frac{i}{2}\left(4890 k^{2}+7823 k-2975\right)(5+k) \hat{A}_{+} V_{+}^{(2)} \\
& -\frac{i}{2}\left(1746 k^{3}+17365 k^{2}+24460 k-7175\right) \hat{A}_{-} U_{-}^{(2)} \\
& -\frac{i\left(519762 k^{5}+3980167 k^{4}+8204637 k^{3}+4284093 k^{2}-1220395 k-739200\right)}{(7 k+3)(23 k+19)} \\
& \times \hat{A}_{3} \hat{T}+\frac{3}{2} i\left(466 k^{3}-819 k^{2}-4260 k-175\right) \hat{A}_{3} T^{(2)} \\
& +\frac{i}{2}\left(4890 k^{2}+7823 k-2975\right)(5+k) \hat{A}_{3} W^{(2)} \\
& +\frac{1}{(k+5)}\left(2022 k^{4}+28451 k^{3}+82512 k^{2}+19815 k-59900\right) \hat{A}_{3} \partial \hat{B}_{3} \\
& -\frac{i}{2}\left(1098 k^{3}+4607 k^{2}+2629 k-2700\right)(k+5) \hat{B}_{+} U_{+}^{(2)} \\
& +\frac{1}{(k+5)}\left(312 k^{4}+5584 k^{3}+19017 k^{2}+9450 k-11575\right) \hat{B}_{+} \partial \hat{B}_{-} \\
& +\frac{i}{2}\left(1098 k^{4}+6953 k^{3}+10756 k^{2}-1235 k-5800\right) \hat{B}_{-} V_{-}^{(2)} \\
& -\frac{1}{(k+5)}\left(312 k^{4}+7156 k^{3}+26471 k^{2}+15290 k-15425\right) \hat{B}_{-} \partial \hat{B}_{+} \\
& -\frac{i}{(7 k+3)(23 k+19)}\left(101016 k^{6}+1081846 k^{5}+4027699 k^{4}\right. \\
& \left.+6039421 k^{3}+2381493 k^{2}-1777935 k-769500\right) \hat{B}_{3} \hat{T} \\
& +\frac{i}{2}\left(1098 k^{4}+3809 k^{3}-4152 k^{2}-12915 k+1900\right) \hat{B}_{3} T^{(2)} \\
& +\frac{i}{2}\left(1098 k^{3}+4607 k^{2}+2629 k-2700\right)(5+k) \hat{B}_{3} W^{(2)} \\
& +\frac{2}{(k+5)}\left(2034 k^{4}+13661 k^{3}+29267 k^{2}+7775 k-18825\right) \hat{B}_{3} \partial \hat{A}_{3} \\
& -\frac{1}{(k+5)}\left(1098 k^{4}+11669 k^{3}+33118 k^{2}+16285 k-17350\right) \hat{B}_{3} \partial \hat{B}_{3} \\
& -\frac{i}{3(k+5)}\left(2994 k^{4}-475 k^{3}-53632 k^{2}-46735 k+40500\right) \partial^{2} \hat{B}_{3} \\
& -\frac{1}{6}\left(1398 k^{3}+2433 k^{2}-4957 k-3500\right)(5+k) \partial^{2} T^{(1)} \\
& -\frac{1}{4}\left(1398 k^{3}+2433 k^{2}-4957 k-3500\right)(5+k) \hat{G}_{11}\left(\hat{G}_{22}-V^{\left(\frac{3}{2}\right)}\right)
\end{aligned}
$$




$$
\begin{aligned}
& +\frac{1}{4}\left(1398 k^{4}+15711 k^{3}+37024 k^{2}-4925 k-32900\right) \hat{G}_{12} \hat{G}_{21} \\
& +\frac{1}{4}\left(1398 k^{3}+2433 k^{2}-4957 k-3500\right)(5+k)\left(\hat{G}_{12} T_{+}^{\left(\frac{3}{2}\right)}+\hat{G}_{21} T_{-}^{\left(\frac{3}{2}\right)}+\hat{G}_{22} U^{\left(\frac{3}{2}\right)}\right) \\
& -\frac{i}{(k+5)}\left(1098 k^{4}+13589 k^{3}+60394 k^{2}+59365 k-27850\right) \hat{A}_{+} \hat{A}_{-} \hat{B}_{3} \\
& -\frac{i}{(k+5)}\left(1098 k^{4}+7301 k^{3}+30578 k^{2}+36005 k-12450\right) \hat{A}_{3} \hat{A}_{3} \hat{B}_{3} \\
& -i\left(1098 k^{3}+4607 k^{2}+2629 k-2700\right)\left(2 \hat{A}_{3} \hat{B}_{+} \hat{B}_{-}+\hat{A}_{3} \hat{B}_{+} \hat{B}_{3}+\hat{B}_{-} \hat{B}_{+} \hat{B}_{3}+\hat{B}_{3} \hat{B}_{3} \hat{B}_{3}\right) \\
& -\frac{1}{2}\left(534 k^{4}+5117 k^{3}+15737 k^{2}+10883 k-7215\right) T^{(1)}\left(\hat{B}_{+} \hat{B}_{-}+\hat{B}_{3} \hat{B}_{3}\right) \\
& \left.+\frac{1}{24}\left(4086 k^{4}+50329 k^{3}+275267 k^{2}+523139 k+300835\right) T^{(1)} T^{(1)} T^{(1)}\right](z) .(6.8)
\end{aligned}
$$

The corresponding algebras between the 10 currents, $\hat{A}_{ \pm}(z), \hat{A}_{3}(z), \hat{B}_{ \pm}(z), \hat{B}_{3}(z)$ and $\hat{G}_{a}(z)$, from large $\mathcal{N}=4$ nonlinear algebra and 16 higher spin currents, $\hat{T}^{(1)}(w), \hat{T}_{ \pm}^{\left(\frac{3}{2}\right)}(w)$, $\hat{T}^{(2)}(w), \hat{U}^{\left(\frac{3}{2}\right)}(w), \hat{U}_{ \pm}^{(2)}(w), \hat{U}^{\left(\frac{5}{2}\right)}(w), \hat{V}^{\left(\frac{3}{2}\right)}(w), \hat{V}_{ \pm}^{(2)}(w), \hat{V}^{\left(\frac{5}{2}\right)}(w), \hat{W}^{(2)}(w), \hat{W}_{ \pm}^{\left(\frac{5}{2}\right)}(w)$ and $\hat{W}^{(3)}(w)$, in $(6.8)$ are given at the end of appendices B and C.

\section{Acknowledgments}

CA would like to thank M.R. Gaberdiel for intensive discussions and R. Gopakumar and H. Kim for discussions. CA would like to thank his previous collaborators K. Schoutens and A. Sevrin for earlier works. This work was supported by the Mid-career Researcher Program through the National Research Foundation of Korea (NRF) grant funded by the Korean government (MEST) (No. 2012-045385/2013-056327). CA acknowledges warm hospitality from the School of Liberal Arts (and Institute of Convergence Fundamental Studies), Seoul National University of Science and Technology.

\section{A The OPEs of the large $\mathcal{N}=4$ nonlinear superconformal algebra from WZW affine currents}

In section 3, the stress tensor is given by (3.2) and the OPE between this stress tensor and itself can be described as

$$
\hat{T}(z) \hat{T}(w)=\frac{1}{(z-w)^{4}} \frac{\hat{c}}{2}+\frac{1}{(z-w)^{2}} 2 \hat{T}(w)+\frac{1}{(z-w)} \partial \hat{T}(w)+\cdots,
$$

where the central charge $\hat{c}$ is given by (3.3).

The 10 currents which are given in (3.14)-(3.17), (3.9) and (3.12) are primary fields under this stress tensor (3.2) as follows:

$$
\hat{T}(z) \Phi(w)=\frac{1}{(z-w)^{2}} h \Phi(w)+\frac{1}{(z-w)} \partial \Phi(w)+\cdots,
$$

where $h=\frac{3}{2}$ for $\Phi=\hat{G}_{a}$ and $h=1$ for $\Phi=\hat{A}_{i}, \hat{B}_{i}$. 
Let us present the nontrivial remaining OPEs between these 10 currents as follows:

- $\operatorname{spin}-\frac{3}{2}$ spin- $\frac{3}{2}$ OPEs

From the explicit expressions in (3.14)-(3.17), the following OPEs can be obtained

$$
\begin{aligned}
\hat{G}_{a}(z) \hat{G}_{b}(w)= & \frac{1}{(z-w)^{3}} \frac{2}{3} c_{\text {Wolf }} \hat{\delta}_{a b}-\frac{1}{(z-w)^{2}} \frac{16}{(5+k)}\left[\hat{k}^{-} \alpha_{a b}^{+i} \hat{A}_{i}+\hat{k}^{+} \alpha_{a b}^{-i} \hat{B}_{i}\right](w) \\
& +\frac{1}{(z-w)}\left[2 \hat{T} \hat{\delta}_{a b}-\frac{1}{2} \frac{16}{(5+k)}\left(\hat{k}^{-} \alpha_{a b}^{+i} \partial \hat{A}_{i}+\hat{k}^{+} \alpha_{a b}^{-i} \partial \hat{B}_{i}\right)+\right. \\
& \left.-\frac{16}{(5+k)}\left(\alpha^{+i} \hat{A}_{i}-\alpha^{-i} \hat{B}_{i}\right)_{c(a}\left(\alpha^{+j} \hat{A}_{j}-\alpha^{-j} \hat{B}_{j}\right)_{b)}^{c}\right](w)+\cdots, \quad \text { (A.3 } 3
\end{aligned}
$$

where $\hat{\delta}_{a b}$ is normalized to be 1 . The central charge $c_{\text {Wolf }}$ appearing above is given by $c_{\text {Wolf }}=\frac{18 k}{(5+k)}(2.3)$ for $N=3$. In general, this central charge is different from the one in the OPE (A.1) of stress energy tensor and itself. Note that compared to the linear case, the nonlinear terms of (A.3) are nontrivial.

- spin-1 spin- $\frac{3}{2}$ OPEs

From the relations (3.9) and the above spin- $\frac{3}{2}$ currents, one obtains the following OPEs between them:

$$
\hat{A}_{i}(z) \hat{G}_{a}(w)=\frac{1}{(z-w)} \alpha_{a b}^{+i} \hat{G}^{b}(w)+\cdots .
$$

Compared to the linear case, the right hand sides of (A.4) look similar to the one in linear case by ignoring the spin- $\frac{1}{2}$ currents over there. Note that the OPEs $\hat{A}_{+}(z) \hat{G}_{11}(w), \hat{A}_{+}(z) \hat{G}_{12}(w), \hat{A}_{-}(z) \hat{G}_{21}(w)$ and $\hat{A}_{-}(z) \hat{G}_{22}(w)$ do not contain the singular terms. This can be understood from the $\mathrm{U}(1)$ charge assignment described in table 1. Furthermore, one has, from (A.4),

$$
\begin{aligned}
& \hat{A}_{\mp}(z)\left(\begin{array}{c}
\hat{G}_{11} \\
\hat{G}_{22}
\end{array}\right)(w)=\mp \frac{1}{(z-w)} i\left(\begin{array}{c}
\hat{G}_{21} \\
\hat{G}_{12}
\end{array}\right)(w)+\cdots, \\
& \hat{A}_{\mp}(z)\left(\begin{array}{c}
\hat{G}_{12} \\
\hat{G}_{21}
\end{array}\right)(w)= \pm \frac{1}{(z-w)} i\left(\begin{array}{c}
\hat{G}_{22} \\
\hat{G}_{11}
\end{array}\right)(w)+\cdots .
\end{aligned}
$$

One can easily check that the $\mathrm{U}(1)$ charge from table 1 is preserved in (A.5).

For the other spin-1 currents (3.10), the similar OPEs can be described as

$$
\hat{B}_{i}(z) \hat{G}_{a}(w)=\frac{1}{(z-w)} \alpha_{a b}^{-i} \hat{G}^{b}(w)+\cdots .
$$

Similarly, the OPEs $\hat{B}_{-}(z) \hat{G}_{11}(w), \hat{B}_{+}(z) \hat{G}_{12}(w), \hat{B}_{-}(z) \hat{G}_{21}(w)$ and $\hat{B}_{+}(z) \hat{G}_{22}(w)$ do not contain the singular terms due to the $\mathrm{U}(1)$ charge conservation. Instead, one has the following singular OPEs, from (A.6),

$$
\hat{B}_{ \pm}(z)\left(\begin{array}{c}
\hat{G}_{11} \\
\hat{G}_{22}
\end{array}\right)(w)= \pm \frac{1}{(z-w)} i\left(\begin{array}{c}
\hat{G}_{12} \\
\hat{G}_{21}
\end{array}\right)(w)+\cdots,
$$




$$
\hat{B}_{\mp}(z)\left(\begin{array}{c}
\hat{G}_{12} \\
\hat{G}_{21}
\end{array}\right)(w)= \pm \frac{1}{(z-w)} i\left(\begin{array}{c}
\hat{G}_{11} \\
\hat{G}_{22}
\end{array}\right)(w)+\cdots .
$$

In this case also, the $\mathrm{U}(1)$ charge from table 1 is preserved in (A.7).

- spin-1 spin-1 OPEs

Finally, the OPEs between the spin-1 currents in (3.9) and (3.10) are as follows:

$$
\begin{aligned}
& \hat{A}_{i}(z) \hat{A}_{j}(w)=-\frac{1}{(z-w)^{2}} \frac{1}{2} \hat{k}^{+} \hat{\delta}_{i j}+\frac{1}{(z-w)} \epsilon_{i j k} \hat{A}_{k}(w) \cdots, \\
& \hat{B}_{i}(z) \hat{B}_{j}(w)=-\frac{1}{(z-w)^{2}} \frac{1}{2} \hat{k}^{-} \hat{\delta}_{i j}+\frac{1}{(z-w)} \epsilon_{i j k} \hat{B}_{k}(w) \cdots,
\end{aligned}
$$

where $\hat{k}^{+}=k$ and $\hat{k}^{-}=3$. One also has, from (A.8), the following OPEs

$$
\begin{aligned}
& \hat{A}_{+}(z) \hat{A}_{-}(w)=\frac{1}{(z-w)^{2}} \hat{k}^{+}-\frac{1}{(z-w)} 2 i \hat{A}_{3}(w)+\cdots \\
& \hat{A}_{ \pm}(z) \hat{A}_{3}(w)= \pm \frac{1}{(z-w)} i \hat{A}_{ \pm}(w)+\cdots \\
& \hat{B}_{+}(z) \hat{B}_{-}(w)=\frac{1}{(z-w)^{2}} \hat{k}^{-}-\frac{1}{(z-w)} 2 i \hat{B}_{3}(w)+\cdots \\
& \hat{B}_{ \pm}(z) \hat{B}_{3}(w)= \pm \frac{1}{(z-w)} i \hat{B}_{ \pm}(w)+\cdots
\end{aligned}
$$

The U(1) charge from table 1 is preserved in (A.9) also.

Therefore, the equations (A.3), (A.4) and (A.6) correspond to the ones in (A.7) in [42] (or (4.3) in [41]). There are also (A.1) and (A.2).

\section{B The OPEs between the large $\mathcal{N}=4$ nonlinear algebra currents and the higher spin currents-I}

In this appendix, we present the OPEs between the six spin- 1 currents in section 3 and the 16 higher spin currents in section 4 .

\section{B.1 The OPEs between six spin-1 currents and the higher spin current of} spins $\left(1, \frac{3}{2}, \frac{3}{2}, 2\right)$

It is easy to see that there are no singular terms in the OPEs $\hat{A}_{\mp}(z) T_{ \pm}^{\left(\frac{3}{2}\right)}(w)$ and $\hat{B}_{\mp}(z) T_{ \pm}^{\left(\frac{3}{2}\right)}(w)$ due to the $\mathrm{U}(1)$ charge conservation. Furthermore, one has the following OPEs,

$$
\begin{aligned}
& \hat{A}_{ \pm}(z) T_{ \pm}^{\left(\frac{3}{2}\right)}(w)=\frac{1}{(z-w)} i\left(\begin{array}{c}
-U^{\left(\frac{3}{2}\right)} \\
V^{\left(\frac{3}{2}\right)}
\end{array}\right)(w)+\cdots \\
& \hat{A}_{3}(z) T_{ \pm}^{\left(\frac{3}{2}\right)}(w)= \pm \frac{1}{(z-w)} \frac{i}{2} T_{ \pm}^{\left(\frac{3}{2}\right)}(w)+\cdots=\hat{B}_{3}(z) T_{ \pm}^{\left(\frac{3}{2}\right)}(w)
\end{aligned}
$$




$$
\hat{B}_{ \pm}(z) T_{ \pm}^{\left(\frac{3}{2}\right)}(w)=\frac{1}{(z-w)} i\left(\begin{array}{c}
\hat{G}_{22}-V^{\left(\frac{3}{2}\right)} \\
\hat{G}_{11}+U^{\left(\frac{3}{2}\right)}
\end{array}\right)(w)+\cdots
$$

One can check the U(1) charge conservation in these OPEs from table 2 .

As before, one obtains the following OPEs as follows: ${ }^{56}$

$$
\begin{aligned}
\hat{A}_{ \pm}(z) T^{(2)}(w)= & -\frac{1}{(z-w)^{2}}\left[\frac{3\left(-3+3 k+2 k^{2}\right)}{(3+7 k)(5+k)}\right] \hat{A}_{ \pm}(w)+\frac{1}{(z-w)} i\left(\begin{array}{c}
U_{-}^{(2)} \\
V_{+}^{(2)}
\end{array}\right)(w) \\
& +\cdots, \\
\hat{A}_{3}(z) T^{(2)}(w)= & \frac{1}{(z-w)^{2}}\left[-\frac{3\left(-3+3 k+2 k^{2}\right)}{(3+7 k)(5+k)} \hat{A}_{3}+\frac{k}{(5+k)} \hat{B}_{3}+\frac{i}{2} T^{(1)}\right](w)+\cdots \\
\hat{B}_{ \pm}(z) T^{(2)}(w)= & \frac{1}{(z-w)^{2}}\left[\frac{(-27+k) k}{(3+7 k)(5+k)}\right] \hat{B}_{ \pm}(w) \\
& +\frac{1}{(z-w)}\left[i\left(\begin{array}{l}
V_{-}^{(2)} \\
U_{+}^{(2)}
\end{array}\right) \mp \frac{4 i}{(5+k)} \hat{A}_{3} \hat{B}_{ \pm}\right](w)+\cdots, \\
\hat{B}_{3}(z) T^{(2)}(w)= & \frac{1}{(z-w)^{2}}\left[\frac{(-27+k) k}{(3+7 k)(5+k)} \hat{B}_{3}+\frac{3}{(5+k)} \hat{A}_{3}+\frac{i}{2} T^{(1)}\right](w)+\cdots
\end{aligned}
$$

The U(1) charge conservation in these OPEs from tables 2 and 3 can be checked again.

\section{B.2 The OPEs between six spin 1 currents and two higher spin currents of spins $\left(\frac{3}{2}, 2,2, \frac{5}{2}\right)$}

One has the following OPEs between the spin-1 currents and the spin- $\frac{3}{2}$ currents:

$$
\begin{aligned}
& \hat{A}_{\mp}(z)\left(\begin{array}{l}
U^{\left(\frac{3}{2}\right)} \\
V^{\left(\frac{3}{2}\right)}
\end{array}\right)(w)=\mp \frac{1}{(z-w)} i T_{ \pm}^{\left(\frac{3}{2}\right)}(w)+\cdots \\
& \hat{A}_{3}(z)\left(\begin{array}{l}
U^{\left(\frac{3}{2}\right)} \\
V^{\left(\frac{3}{2}\right)}
\end{array}\right)(w)=\frac{1}{(z-w)} \frac{i}{2}\left(\begin{array}{c}
-U^{\left(\frac{3}{2}\right)} \\
V^{\left(\frac{3}{2}\right)}
\end{array}\right)(w)+\cdots=-\hat{B}_{3}(z)\left(\begin{array}{c}
U^{\left(\frac{3}{2}\right)} \\
V^{\left(\frac{3}{2}\right)}
\end{array}\right)(w) \\
& \left.\hat{B}_{ \pm}(z)\left(\begin{array}{l}
U^{\left(\frac{3}{2}\right)} \\
V^{\left(\frac{3}{2}\right)}
\end{array}\right)(w)=\frac{1}{(z-w)} i\left[\begin{array}{l}
\left(\frac{3}{2}\right) \\
\mp
\end{array}\right)\left(\begin{array}{c}
\hat{G}_{12} \\
\hat{G}_{21}
\end{array}\right)\right](w)+\cdots
\end{aligned}
$$

One can check the U(1) charge conservation from table 2 .

As performed before, one has the OPEs between the spin-1 currents and the spin-2 currents,

$$
\begin{aligned}
& \hat{A}_{+}(z)\left(\begin{array}{c}
U_{+}^{(2)} \\
V_{-}^{(2)}
\end{array}\right)(w)=\frac{1}{(z-w)} \frac{2}{(5+k)}\left[\mp i \hat{A}_{+} \hat{B}_{\mp}\right](w)+\cdots, \\
& \hat{A}_{-}(z)\left(\begin{array}{l}
U_{+}^{(2)} \\
V_{-}^{(2)}
\end{array}\right)(w)=\frac{1}{(z-w)} \frac{2}{(5+k)}\left[ \pm i \hat{A}_{-} \hat{B}_{\mp}\right](w)+\cdots,
\end{aligned}
$$

\footnotetext{
${ }^{56}$ The spin- 2 current $T^{(2)}(z)$ is not to be confused with the spin-2 stress tensor $\hat{T}(z)$ in $(3.2)$.
} 


$$
\begin{aligned}
\hat{A}_{3}(z)\left(\begin{array}{c}
U_{+}^{(2)} \\
V_{-}^{(2)}
\end{array}\right)(w)= & \pm \frac{1}{(z-w)^{2}} \frac{k}{(5+k)}\left[\hat{B}_{\mp}\right](w)+\cdots \\
\hat{B}_{ \pm}(z)\left(\begin{array}{c}
U_{+}^{(2)} \\
V_{-}^{(2)}
\end{array}\right)(w)= & \frac{1}{(z-w)^{2}}\left[ \pm \frac{6}{(5+k)} \hat{A}_{3} \mp i T^{(1)}\right](w) \\
& +\frac{1}{(z-w)}\left[-\frac{2 i\left(15+23 k+4 k^{2}\right)}{(3+7 k)(5+k)} \hat{T}+i T^{(2)}+i W^{(2)}\right. \\
& \left.+\frac{2 i}{(5+k)}\left(-\hat{A}_{i} \hat{A}_{i}+2 \hat{A}_{3} \hat{B}_{3}-\hat{B}_{i} \hat{B}_{i}\right)\right](w)+\cdots \\
\hat{B}_{3}(z)\left(\begin{array}{l}
U_{+}^{(2)} \\
V_{-}^{(2)}
\end{array}\right)(w)= & \frac{1}{(z-w)} i\left(\begin{array}{c}
U_{+}^{(2)} \\
-V_{-}^{(2)}
\end{array}\right)(w)+\cdots
\end{aligned}
$$

One can check the U(1) charge conservation in these OPEs from tables 2 and 3.

Furthermore, the remaining OPEs can be rewritten as

$$
\begin{aligned}
\hat{A}_{\mp}(z)\left(\begin{array}{c}
U_{-}^{(2)} \\
V_{+}^{(2)}
\end{array}\right)(w)= & \frac{1}{(z-w)^{2}}\left[ \pm \frac{2 k}{(5+k)} \hat{B}_{3} \pm i T^{(1)}\right](w) \\
& +\frac{1}{(z-w)}\left[\frac{6 i k}{(3+7 k)} \hat{T}+i T^{(2)}-i W^{(2)}\right](w)+\cdots \\
\hat{A}_{3}(z)\left(\begin{array}{l}
U_{-}^{(2)} \\
V_{+}^{(2)}
\end{array}\right)(w)= & \frac{1}{(z-w)} i\left(\begin{array}{c}
-U_{-}^{(2)} \\
V_{+}^{(2)}
\end{array}\right)(w)+\cdots \\
\hat{B}_{ \pm}(z)\left(\begin{array}{l}
U_{-}^{(2)} \\
V_{+}^{(2)}
\end{array}\right)(w)= & \frac{1}{(z-w)} \frac{2}{(5+k)}\left[-i \hat{A}_{ \pm} \hat{B}_{ \pm}\right](w)+\cdots \\
\hat{B}_{\mp}(z)\left(\begin{array}{l}
U_{-}^{(2)} \\
V_{+}^{(2)}
\end{array}\right)(w)= & \frac{1}{(z-w)} \frac{2}{(5+k)}\left[i \hat{A}_{ \pm} \hat{B}_{\mp}\right](w)+\cdots \\
\hat{B}_{3}(z)\left(\begin{array}{l}
U_{-}^{(2)} \\
V_{+}^{(2)}
\end{array}\right)(w)= & \pm \frac{1}{(z-w)^{2}} \frac{3}{(5+k)}\left[\hat{A}_{ \pm}\right](w)+\cdots
\end{aligned}
$$

One can check the U(1) charge conservation in these OPEs from tables 2 and 3.

The following OPEs between the spin- 1 currents and the spin- $\frac{5}{2}$ current can be obtained as

$$
\begin{aligned}
\hat{A}_{+}(z) U^{\left(\frac{5}{2}\right)}(w)= & \frac{1}{(z-w)} \frac{1}{(5+k)}\left[2 \hat{A}_{+}\left(\hat{G}_{11}+U^{\left(\frac{3}{2}\right)}\right)\right](w)+\cdots, \\
\hat{A}_{-}(z) U^{\left(\frac{5}{2}\right)}(w)= & \frac{1}{(z-w)^{2}}\left[\frac{2 i(9+k)}{3(5+k)} \hat{G}_{21}+\frac{2 i(19+5 k)}{3(5+k)} T_{+}^{\left(\frac{3}{2}\right)}\right](w) \\
& +\frac{1}{(z-w)}\left[-i W_{+}^{\left(\frac{5}{2}\right)}-\frac{4 i}{3(5+k)} \partial \hat{G}_{21}-\frac{4 i}{3(5+k)} \partial T_{+}^{\left(\frac{3}{2}\right)}\right. \\
& \left.+\frac{1}{(5+k)}\left(-2 \hat{A}_{-}\left(\hat{G}_{11}+U^{\left(\frac{3}{2}\right)}\right)-2 \hat{B}_{-} V^{\left(\frac{3}{2}\right)}\right)\right](w)+\cdots, \\
\hat{A}_{3}(z) U^{\left(\frac{5}{2}\right)}(w)= & \frac{1}{(z-w)^{2}}\left[\frac{2 i(3+2 k)}{3(5+k)} \hat{G}_{11}+\frac{8 i(2+k)}{3(5+k)} U^{\left(\frac{3}{2}\right)}\right](w)-\frac{1}{(z-w)} \frac{i}{2} U^{\left(\frac{5}{2}\right)}+\cdots,
\end{aligned}
$$




$$
\begin{aligned}
\hat{B}_{+}(z) U^{\left(\frac{5}{2}\right)}(w)= & \frac{1}{(z-w)^{2}}\left[-\frac{4 i(1+k)}{3(5+k)} \hat{G}_{12}+\frac{8 i(2+k)}{3(5+k)} T_{-}^{\left(\frac{3}{2}\right)}\right](w) \\
& +\frac{1}{(z-w)}\left[-i W_{-}^{\left(\frac{5}{2}\right)}-\frac{8 i}{3(5+k)} \partial \hat{G}_{12}+\frac{4 i}{3(5+k)} \partial T_{-}^{\left(\frac{3}{2}\right)}\right. \\
& \left.+\frac{1}{(5+k)}\left(2 \hat{A}_{+} \hat{G}_{22}-4 \hat{A}_{3}\left(\hat{G}_{12}-T_{-}^{\left(\frac{3}{2}\right)}\right)+2 \hat{B}_{+}\left(\hat{G}_{11}-U^{\left(\frac{3}{2}\right)}\right)\right)\right](w)+\cdots, \\
\hat{B}_{-}(z) U^{\left(\frac{5}{2}\right)}(w)= & -\frac{1}{(z-w)} \frac{1}{(5+k)}\left[2 \hat{B}_{-} \hat{G}_{11}\right](w)+\cdots, \\
\hat{B}_{3}(z) U^{\left(\frac{5}{2}\right)}(w)= & \frac{1}{(z-w)^{2}}\left[\frac{2 i(6+k)}{3(5+k)} \hat{G}_{11}+\frac{4 i(2+k)}{3(5+k)} U^{\left(\frac{3}{2}\right)}\right](w)+\frac{1}{(z-w)} \frac{i}{2} U^{\left(\frac{5}{2}\right)}(w)+\cdots .
\end{aligned}
$$

One can check the U(1) charge conservation in these OPEs from tables 2 and 4.

Then the following nontrivial remaining OPEs hold as follows:

$$
\begin{aligned}
\hat{A}_{+}(z) V^{\left(\frac{5}{2}\right)}(w)= & \frac{1}{(z-w)^{2}}\left[-\frac{2 i(9+k)}{3(5+k)} \hat{G}_{12}+\frac{4 i(7+k)}{3(5+k)} T_{-}^{\left(\frac{3}{2}\right)}\right](w) \\
& +\frac{1}{(z-w)}\left[i W_{-}^{\left(\frac{5}{2}\right)}+\frac{4 i}{3(5+k)} \partial \hat{G}_{12}+\frac{4 i}{3(5+k)} \partial T_{-}^{\left(\frac{3}{2}\right)}\right. \\
& \left.+\frac{1}{(5+k)}\left(-2 \hat{A}_{+}\left(V^{\left(\frac{3}{2}\right)}+\hat{G}_{22}\right)-2 \hat{B}_{+} \hat{G}_{11}+4 \hat{B}_{3} T_{-}^{\left(\frac{3}{2}\right)}\right)\right](w)+\cdots, \\
\hat{A}_{-}(z) V^{\left(\frac{5}{2}\right)}(w)= & \frac{1}{(z-w)} \frac{1}{(5+k)}\left[2 \hat{A}_{-} \hat{G}_{22}\right](w)+\cdots, \\
\hat{A}_{3}(z) V^{\left(\frac{5}{2}\right)}(w)= & \frac{1}{(z-w)^{2}}\left[-\frac{2 i(3+2 k)}{3(5+k)} \hat{G}_{22}+\frac{2 i(7+k)}{3(5+k)} V^{\left(\frac{3}{2}\right)}\right](w)+\frac{1}{(z-w)} \frac{i}{2} V^{\left(\frac{5}{2}\right)}(w), \\
\hat{B}_{+}(z) V^{\left(\frac{5}{2}\right)}(w)= & \frac{1}{(z-w)} \frac{1}{(5+k)}\left[-2 \hat{B}_{+}\left(\hat{G}_{22}-V^{\left(\frac{3}{2}\right)}\right)\right](w)+\cdots, \\
\hat{B}_{-}(z) V^{\left(\frac{5}{2}\right)}(w)= & \frac{1}{(z-w)^{2}}\left[\frac{4 i(8+k)}{3(5+k)} \hat{G}_{21}+\frac{4 i(11+2 k)}{3(5+k)} T_{+}^{\left(\frac{3}{2}\right)}\right](w) \\
& +\frac{1}{(z-w)}\left[i W_{+}^{\left(\frac{5}{2}\right)}-\frac{4 i}{3(5+k)} \partial T_{+}^{\left(\frac{3}{2}\right)}\right. \\
& \left.+\frac{1}{(5+k)}\left(-2 \hat{A}_{-} U^{\left(\frac{3}{2}\right)}+2 \hat{B}_{-}\left(\hat{G}_{22}-V^{\left(\frac{3}{2}\right)}\right)\right)\right](w)+\cdots, \\
\hat{B}_{3}(z) V^{\left(\frac{5}{2}\right)}(w)= & \frac{1}{(z-w)^{2}}\left[-\frac{2 i(6+k)}{3(5+k)} \hat{G}_{22}+\frac{4 i(7+k)}{3(5+k)} V^{\left(\frac{3}{2}\right)}\right](w)-\frac{1}{(z-w)} \frac{i}{2} V^{\left(\frac{5}{2}\right)}(w)
\end{aligned}
$$

One can check the $\mathrm{U}(1)$ charge conservation in these OPEs from tables 2 and 4 .

\section{B.3 The OPEs between six spin 1 currents and the higher spin current of spins $\left(2, \frac{5}{2}, \frac{5}{2}, 3\right)$}

The nontrivial OPEs between the spin- 1 currents and the spin- 2 current can be described as

$$
\hat{A}_{ \pm}(z) W^{(2)}(w)=\frac{1}{(z-w)^{2}} \frac{3}{(5+k)} \hat{A}_{ \pm}(w)-\frac{1}{(z-w)} i\left(\begin{array}{c}
U_{-}^{(2)} \\
V_{+}^{(2)}
\end{array}\right)(w)+\cdots
$$




$$
\begin{aligned}
& \hat{A}_{3}(z) W^{(2)}(w)=\frac{1}{(z-w)^{2}}\left[\frac{3}{(5+k)} \hat{A}_{3}-\frac{k}{(5+k)} \hat{B}_{3}-\frac{i}{2} T^{(1)}\right](w)+\cdots \\
& \hat{B}_{ \pm}(z) W^{(2)}(w)=\frac{1}{(z-w)^{2}} \frac{k}{(5+k)} \hat{B}_{ \pm}(w)+\frac{1}{(z-w)} i\left(\begin{array}{c}
V_{-}^{(2)} \\
U_{+}^{(2)}
\end{array}\right)(w)+\cdots \\
& \hat{B}_{3}(z) W^{(2)}(w)=\frac{1}{(z-w)^{2}}\left[-\frac{3}{(5+k)} \hat{A}_{3}+\frac{k}{(5+k)} \hat{B}_{3}+\frac{i}{2} T^{(1)}\right](w)+\cdots
\end{aligned}
$$

One can write the OPEs between the spin- 1 currents and the spin- $\frac{5}{2}$ currents as follows:

$$
\begin{aligned}
\hat{A}_{+}(z) W_{+}^{\left(\frac{5}{2}\right)}(w)= & \frac{1}{(z-w)^{2}}\left[\frac{2 i}{3} \hat{G}_{11}+\frac{10 i(3+k)}{3(5+k)} U^{\left(\frac{3}{2}\right)}\right](w) \\
& +\frac{1}{(z-w)}\left[\frac{4 i}{3(5+k)} \partial \hat{G}_{11}+\frac{4 i}{3(5+k)} \partial U^{\left(\frac{3}{2}\right)}-i U^{\left(\frac{5}{2}\right)}\right. \\
& \left.+\frac{1}{(5+k)}\left(2 \hat{A}_{+}\left(\hat{G}_{21}+T_{+}^{\left(\frac{3}{2}\right)}\right)-2 \hat{B}_{-} T_{-}^{\left(\frac{3}{2}\right)}\right)\right](w)+\cdots \\
\hat{A}_{\mp}(z) W_{ \pm}^{\left(\frac{5}{2}\right)}(w)= & -\frac{1}{(z-w)} \frac{2}{(5+k)} \hat{A}_{\mp}\left(\begin{array}{c}
\hat{G}_{21}+T_{+}^{\left(\frac{3}{2}\right)} \\
\hat{G}_{12}-T_{-}^{\left(\frac{3}{2}\right)}
\end{array}\right)(w)+\cdots \\
\hat{A}_{3}(z) W_{+}^{\left(\frac{5}{2}\right)}(w)= & \frac{1}{(z-w)^{2}}\left[\frac{2 i(-4+k)}{3(5+k)} \hat{G}_{21}-\frac{2 i(9+k)}{3(5+k)} T_{+}^{\left(\frac{3}{2}\right)}\right](w) \\
& +\frac{1}{(z-w)} \frac{i}{2} W_{+}^{\left(\frac{5}{2}\right)}(w)+\cdots, \\
\hat{B}_{+}(z) W_{+}^{\left(\frac{5}{2}\right)}(w)= & \frac{1}{(z-w)^{2}}\left[\frac{4 i(1+k)}{3(5+k)} \hat{G}_{22}-\frac{4 i(9+2 k)}{3(5+k)} V^{\left(\frac{3}{2}\right)}\right](w) \\
& +\frac{1}{(z-w)}\left[\frac{4 i}{3(5+k)} \partial \hat{G}_{22}-\frac{4 i}{3(5+k)} \partial V^{\left(\frac{3}{2}\right)}+i V^{\left(\frac{5}{2}\right)}\right. \\
& \left.+\frac{1}{(5+k)}\left(-2 \hat{A}_{-}\left(\hat{G}_{12}+T_{-}^{\left(\frac{3}{2}\right)}\right)-2 \hat{B}_{+} T_{+}^{\left(\frac{3}{2}\right)}\right)\right](w)+\cdots, \\
\hat{B}_{\mp}(z) W_{ \pm}^{\left(\frac{5}{2}\right)}(w)= & \frac{1}{(z-w)} \frac{1}{(5+k)}\left[ \pm 2 \hat{B}_{\mp} T_{ \pm}^{\left(\frac{3}{2}\right)}\right](w)+\cdots \\
\hat{B}_{3}(z) W_{+}^{\left(\frac{5}{2}\right)}(w)= & \frac{1}{(z-w)^{2}}\left[\frac{2 i(8+k)}{3(5+k)} \hat{G}_{21}+\frac{4 i(3+k)}{3(5+k)} T_{+}^{\left(\frac{3}{2}\right)}\right](w)+\frac{1}{(z-w)} \frac{i}{2} W_{+}^{\left(\frac{5}{2}\right)}(w)+\cdots
\end{aligned}
$$

In this case, the four remaining OPEs can be rewritten as

$$
\begin{aligned}
\hat{A}_{-}(z) W_{-}^{\left(\frac{5}{2}\right)}(w)= & \frac{1}{(z-w)^{2}}\left[-\frac{2 i}{3} \hat{G}_{22}+\frac{10 i(3+k)}{3(5+k)} V^{\left(\frac{3}{2}\right)}\right](w) \\
& +\frac{1}{(z-w)}\left[-\frac{4 i}{3(5+k)} \partial \hat{G}_{22}-\frac{4 i}{3(5+k)} \partial V^{\left(\frac{3}{2}\right)}+i V^{\left(\frac{5}{2}\right)}\right. \\
& \left.+\frac{1}{(5+k)}\left(2 \hat{A}_{-} \hat{G}_{12}+4 \hat{A}_{3} V^{\left(\frac{3}{2}\right)}-2 \hat{B}_{+} \hat{G}_{21}-4 \hat{B}_{3} V^{\left(\frac{3}{2}\right)}\right)\right](w)+\cdots, \\
\hat{A}_{3}(z) W_{-}^{\left(\frac{5}{2}\right)}(w)= & \frac{1}{(z-w)^{2}}\left[-\frac{2 i(-4+k)}{3(5+k)} \hat{G}_{12}-\frac{2 i(9+k)}{3(5+k)} T_{-}^{\left(\frac{3}{2}\right)}\right](w)-\frac{1}{(z-w)} \frac{i}{2} W_{-}^{\left(\frac{5}{2}\right)}(w) \\
& +\cdots,
\end{aligned}
$$




$$
\begin{aligned}
\hat{B}_{-}(z) W_{-}^{\left(\frac{5}{2}\right)}(w)= & \frac{1}{(z-w)^{2}}\left[-\frac{4 i(1+k)}{3(5+k)} \hat{G}_{11}-\frac{4 i(9+2 k)}{3(5+k)} U^{\left(\frac{3}{2}\right)}\right](w) \\
& +\frac{1}{(z-w)}\left[-\frac{4 i}{3(5+k)} \partial \hat{G}_{11}+\frac{4 i}{3(5+k)} \partial U^{\left(\frac{3}{2}\right)}-i U^{\left(\frac{5}{2}\right)}\right. \\
& \left.+\frac{1}{(5+k)}\left(-2 \hat{A}_{+} \hat{G}_{21}+4 \hat{A}_{3} U^{\left(\frac{3}{2}\right)}+2 \hat{B}_{-} \hat{G}_{12}-4 \hat{B}_{3} U^{\left(\frac{3}{2}\right)}\right)\right](w)+\cdots, \\
\hat{B}_{3}(z) W_{-}^{\left(\frac{5}{2}\right)}(w)= & \frac{1}{(z-w)^{2}}\left[-\frac{2 i(8+k)}{3(5+k)} \hat{G}_{12}+\frac{4 i(3+k)}{3(5+k)} T_{-}^{\left(\frac{3}{2}\right)}\right](w)-\frac{1}{(z-w)} \frac{i}{2} W_{-}^{\left(\frac{5}{2}\right)}(w)
\end{aligned}
$$

Finally the six nontrivial OPEs between the spin- 1 currents and the spin-3 current can be described as

$$
\begin{aligned}
& \hat{A}_{+}(z) W^{(3)}(w)=\frac{1}{(z-w)^{3}}\left[-\frac{2\left(35-20 k+33 k^{2}\right)}{(5+k)^{2}(19+23 k)}\right] \hat{A}_{+}(w) \\
& +\frac{1}{(z-w)^{2}}\left[-\frac{(13+2 k)}{(5+k)} i U_{-}^{(2)}-\frac{6\left(1+41 k+12 k^{2}\right)}{(5+k)^{2}(19+23 k)} i \hat{A}_{+} \hat{A}_{3}\right. \\
& -\frac{2\left(76+311 k+63 k^{2}\right)}{(5+k)^{2}(19+23 k)} i \hat{A}_{+} \hat{B}_{3}+\frac{8}{(5+k)^{2}} i \hat{A}_{+} \hat{B}_{3} \\
& \left.-\frac{\left(-32+143 k+3 k^{2}\right)}{(5+k)^{2}(19+23 k)} \partial \hat{A}_{+}-\frac{\left(-139-7 k+8 k^{2}\right)}{(5+k)(19+23 k)} T^{(1)} \hat{A}_{+}\right](w) \\
& +\frac{1}{(z-w)}\left[\frac{1}{(5+k)} T^{(1)} \partial \hat{A}_{+}-\frac{8\left(48+97 k+29 k^{2}\right)}{(3+7 k)(19+23 k)(5+k)} \hat{A}_{+} \hat{T}\right. \\
& +\frac{4}{(5+k)} \hat{A}_{+} T^{(2)}+\frac{2}{(5+k)^{2}} i \hat{A}_{+} \partial \hat{A}_{3}+\frac{2 k}{(5+k)^{2}} i \hat{A}_{+} \partial \hat{B}_{3} \\
& -\frac{1}{(5+k)} \hat{A}_{+} \partial T^{(1)}-\frac{2}{(5+k)^{2}} i \hat{A}_{3} \partial \hat{A}_{+}-\frac{4}{(5+k)} \hat{B}_{3} U_{-}^{(2)} \\
& -\frac{2(-2+k)}{(5+k)^{2}} i \hat{B}_{3} \partial \hat{A}_{+}-\frac{1}{(5+k)} i \partial U_{-}^{(2)}-\frac{2}{(5+k)} i \hat{G}_{11} \hat{G}_{12} \\
& -\frac{4}{(5+k)^{2}} \hat{A}_{+} \hat{A}_{+} \hat{A}_{-}+\frac{16}{(5+k)^{2}} \hat{A}_{+} \hat{A}_{3} \hat{B}_{3}-\frac{8}{(5+k)^{2}} \hat{A}_{+} \hat{B}_{3} \hat{B}_{3} \\
& \left.-\frac{4}{(5+k)^{2}} \hat{A} \hat{A}_{4} \hat{A}_{+}-\frac{8}{(5+k)^{2}} \hat{A}_{3} \hat{A}_{4} \hat{A}_{3}-\frac{4}{(5+k)^{2}} \hat{B}_{-} \hat{A}_{+} \hat{B}_{+}\right](w)+\cdots \text {, } \\
& \hat{A}_{-}(z) W^{(3)}(w)=\frac{1}{(z-w)^{3}}\left[\frac{2\left(35-20 k+33 k^{2}\right)}{(5+k)^{2}(19+23 k)}\right] \hat{A}_{-}(w) \\
& +\frac{1}{(z-w)^{2}}\left[\frac{(15+4 k)}{(5+k)} i V_{+}^{(2)}-\frac{6\left(1+41 k+12 k^{2}\right)}{(5+k)^{2}(19+23 k)} i \hat{A}_{-} \hat{A}_{3}\right. \\
& -\frac{2\left(76+311 k+63 k^{2}\right)}{(5+k)^{2}(19+23 k)} i \hat{A}_{-} \hat{B}_{3}-\frac{8}{(5+k)^{2}} i \hat{A}_{-} \hat{B}_{3} \\
& \left.+\frac{\left(-32+143 k+3 k^{2}\right)}{(5+k)^{2}(19+23 k)} \partial \hat{A}_{-}-\frac{\left(-139-7 k+8 k^{2}\right)}{(5+k)(19+23 k)} T^{(1)} \hat{A}_{-}\right](w) \\
& +\frac{1}{(z-w)}\left[-\frac{1}{(5+k)} T^{(1)} \partial \hat{A}_{-}-\frac{2}{(5+k)} \hat{A}_{-} W^{(2)}\right.
\end{aligned}
$$




$$
\begin{aligned}
& +\frac{4\left(96+251 k+127 k^{2}\right)}{(3+7 k)(19+23 k)(5+k)} \hat{A}_{-} \hat{T}-\frac{2}{(5+k)} \hat{A}_{-} T^{(2)}+\frac{18}{(5+k)^{2}} i \hat{A}_{-} \partial \hat{A}_{3} \\
& -\frac{2 k}{(5+k)^{2}} i \hat{A}_{-} \partial \hat{B}_{3}+\frac{1}{(5+k)} \hat{A}_{-} \partial T^{(1)}+\frac{6}{(5+k)^{2}} i \hat{A}_{3} \partial \hat{A}_{-} \\
& +\frac{4}{(5+k)} \hat{A}_{3} V_{+}^{(2)}-\frac{1}{(5+k)} i \partial V_{+}^{(2)}+\frac{2}{(5+k)} i \hat{G}_{21} \hat{G}_{22} \\
& +\frac{8}{(5+k)^{2}} \hat{A}_{+} \hat{A}_{-} \hat{A}_{-}+\frac{8}{(5+k)^{2}} \hat{A}_{-} \hat{A}_{3} \hat{A}_{3}-\frac{2(-2+k)}{(5+k)^{2}} \hat{A}_{-} \hat{A}_{3} \hat{B}_{3} \\
& +\frac{8}{(5+k)^{2}} \hat{A}_{-} \hat{B}_{3} \hat{B}_{3}+\frac{2(-10+k)}{(5+k)^{2}} \hat{A}_{3} \hat{A}_{-} \hat{B}_{3} \\
& \left.+\frac{4}{(5+k)^{2}} \hat{B}_{-} \hat{A}_{-} \hat{B}_{+}-\frac{4}{(5+k)} \hat{B}_{3} V_{+}^{(2)}\right](w)+\cdots, \\
& \hat{A}_{3}(z) W^{(3)}(w)=\frac{1}{(z-w)^{3}}\left[\frac{2(-3+k) k}{(5+k)^{2}} \hat{B}_{3}+\frac{(1+k)}{(5+k)} i T^{(1)}\right](w) \\
& +\frac{1}{(z-w)^{2}}\left[-\frac{2\left(-57+350 k+699 k^{2}\right)}{(3+7 k)(19+23 k)(5+k)} i \hat{T}-\frac{8}{(5+k)} i T^{(2)}\right. \\
& -\frac{4(-2+k)}{(5+k)^{2}} i \hat{A}_{1} \hat{A}_{1}-\frac{4(-2+k)}{(5+k)^{2}} i \hat{A}_{2} \hat{A}_{2}-\frac{2\left(-73+69 k+82 k^{2}\right)}{(5+k)^{2}(19+23 k)} i \hat{A}_{3} \hat{A}_{3} \\
& +\frac{4\left(-76-173 k+3 k^{2}\right)}{(5+k)^{2}(19+23 k)} \hat{A}_{3} \hat{B}_{3}-\frac{2(-1+k)}{(5+k)^{2}} i \hat{B}_{1} \hat{B}_{1}-\frac{2(-1+k)}{(5+k)^{2}} i \hat{B}_{2} \hat{B}_{2} \\
& +\frac{2}{(5+k)^{2}} i \hat{B}_{3} \hat{B}_{3}-\frac{(-3+k) k}{(5+k)^{2}} \partial \hat{B}_{3}-\frac{(1+k)}{2(5+k)} i \partial T^{(1)}+\frac{2(4+k)}{(5+k)} i W^{(2)} \\
& \left.-\frac{2\left(-79-15 k+4 k^{2}\right)}{(5+k)(19+23 k)} T^{(1)} \hat{A}_{3}-\frac{2}{(5+k)} T^{(1)} \hat{B}_{3}\right](w)+\cdots, \\
& \hat{B}_{+}(z) W^{(3)}(w)=\frac{1}{(z-w)^{3}}\left[-\frac{4 k(-167+101 k)}{3(5+k)^{2}(19+23 k)}\right] \hat{B}_{+}(w) \\
& +\frac{1}{(z-w)^{2}}\left[\frac{(7+4 k)}{(5+k)} i V_{-}^{(2)}-\frac{2\left(79+253 k+82 k^{2}\right)}{(5+k)^{2}(19+23 k)} i \hat{A}_{3} \hat{B}_{+}\right. \\
& -\frac{2(1+k)}{(5+k)^{2}} i \hat{A}_{3} \hat{B}_{+}-\frac{2 k(181+17 k)}{(5+k)^{2}(19+23 k)} i \hat{B}_{+} \hat{B}_{3} \\
& \left.-\frac{k(-877+151 k)}{3(5+k)^{2}(19+23 k)} \partial \hat{B}_{+}-\frac{\left(-101+39 k+8 k^{2}\right)}{(5+k)(19+23 k)} T^{(1)} \hat{B}_{+}\right](w) \\
& +\frac{1}{(z-w)}\left[-\frac{1}{(5+k)} T^{(1)} \partial \hat{B}_{+}-\frac{8(-27+k) k}{(3+7 k)(19+23 k)(5+k)} \hat{B}_{+} \hat{T}\right. \\
& -\frac{4}{(5+k)} \hat{B}_{+} T^{(2)}-\frac{2(2+k)}{(5+k)^{2}} i \hat{B}_{+} \partial \hat{A}_{3}+\frac{14 k}{3(5+k)^{2}} i \hat{B}_{+} \partial \hat{B}_{3} \\
& +\frac{1}{(5+k)} \hat{B}_{+} \partial T^{(1)}+\frac{4}{(5+k)} \hat{A}_{3} V_{-}^{(2)}+\frac{1}{(5+k)} i \partial V_{-}^{(2)} \\
& +\frac{5 k}{3(5+k)^{2}} \hat{B}_{+} \hat{B}_{+} \hat{B}_{-}+\frac{4}{(5+k)^{2}} \hat{A}_{-} \hat{A}_{+} \hat{B}_{+}+\frac{2(-8+k)}{(5+k)^{2}} \hat{A}_{3} \hat{B}_{+} \hat{B}_{3}
\end{aligned}
$$




$$
\begin{aligned}
& +\frac{2 k}{(5+k)^{2}} \hat{B}_{+} \hat{B}_{3} \hat{B}_{3}-\frac{5 k}{3(5+k)^{2}} \hat{B}_{-} \hat{B}_{+} \hat{B}_{+}-\frac{2(-8+k)}{(5+k)^{2}} \hat{B}_{3} \hat{A}_{3} \hat{B}_{+} \\
& \left.-\frac{2 k}{(5+k)^{2}} \hat{B}_{3} \hat{B}_{+} \hat{B}_{3}+\frac{2}{(5+k)} i \hat{G}_{12} \hat{G}_{22}\right](w)+\cdots, \\
& \hat{B}_{-}(z) W^{(3)}(w)=\frac{1}{(z-w)^{3}}\left[\frac{4 k(-167+101 k)}{3(5+k)^{2}(19+23 k)}\right] \hat{B}_{-}(w) \\
& +\frac{1}{(z-w)^{2}}\left[-\frac{(15+4 k)}{(5+k)} i U_{+}^{(2)}-\frac{2\left(79+253 k+82 k^{2}\right)}{(5+k)^{2}(19+23 k)} i \hat{A}_{3} \hat{B}_{-}\right. \\
& +\frac{2(1+k)}{(5+k)^{2}} i \hat{A}_{3} \hat{B}_{-}-\frac{2 k(181+17 k)}{(5+k)^{2}(19+23 k)} i \hat{B}_{-} \hat{B}_{3} \\
& \left.+\frac{k(-877+151 k)}{3(5+k)^{2}(19+23 k)} \partial \hat{B}_{-}-\frac{\left(-101+39 k+8 k^{2}\right)}{(5+k)(19+23 k)} T^{(1)} \hat{B}_{-}\right](w) \\
& +\frac{1}{(z-w)}\left[-\frac{2 k}{(5+k)^{2}} i \hat{A}_{3} \partial \hat{B}_{-}+\frac{4}{(5+k)} \hat{A}_{3} U_{+}^{(2)}+\frac{4(-3+2 k)}{3(5+k)^{2}} \partial^{2} \hat{B}_{-}\right. \\
& +\frac{4\left(57+199 k+90 k^{2}\right)}{(3+7 k)(19+23 k)(5+k)} \hat{B}_{-} \hat{T}+\frac{2}{(5+k)} \hat{B}_{-} T^{(2)} \\
& +\frac{2 k}{(5+k)^{2}} i \hat{B}_{-} \partial \hat{A}_{3}-\frac{2(-6+k)}{3(5+k)^{2}} i \hat{B}_{-} \partial \hat{B}_{3}-\frac{1}{(5+k)} \hat{B}_{-} \partial T^{(1)} \\
& -\frac{4}{(5+k)} \hat{B}_{3} U_{+}^{(2)}+\frac{1}{(5+k)} i \partial U_{+}^{(2)}+\frac{2 k}{(5+k)^{2}} i \hat{B}_{3} \partial \hat{B}_{-} \\
& -\frac{2}{(5+k)} i \hat{G}_{11} \hat{G}_{21}+\frac{4}{(5+k)^{2}} \hat{A}_{3} \hat{A}_{3} \hat{B}_{-}-\frac{2}{(5+k)} \hat{B}_{-} W^{(2)} \\
& -\frac{8}{(5+k)^{2}} \hat{A}_{3} \hat{B}_{-} \hat{B}_{3}+\frac{4}{(5+k)^{2}} \hat{B}_{-} \hat{B}_{3} \hat{B}_{3}+\frac{4}{(5+k)^{2}} \hat{B}_{+} \hat{B}_{-} \hat{B}_{-} \\
& \left.+\frac{1}{(5+k)} i T^{(1)} \hat{B}_{-} \hat{B}_{3}-\frac{1}{(5+k)} i \hat{B}_{3} T^{(1)} \hat{B}_{-}\right](w)+\cdots, \\
& \hat{B}_{3}(z) W^{(3)}(w)=\frac{1}{(z-w)^{3}}\left[-\frac{6(-3+k)}{(5+k)^{2}} \hat{A}_{3}-\frac{4}{(5+k)} i T^{(1)}\right](w) \\
& +\frac{1}{(z-w)^{2}}\left[-\frac{2\left(399+1234 k+859 k^{2}+184 k^{3}\right)}{(3+7 k)(19+23 k)(5+k)} i \hat{T}+\frac{2(1+k)}{(5+k)} i T^{(2)}\right. \\
& -\frac{4(2+k)}{(5+k)^{2}} i \hat{A}_{1} \hat{A}_{1}-\frac{4(2+k)}{(5+k)^{2}} i \hat{A}_{2} \hat{A}_{2}-\frac{2(1+2 k)}{(5+k)^{2}} i \hat{A}_{3} \hat{A}_{3}+\frac{2(4+k)}{(5+k)} i W^{(2)} \\
& +\frac{4\left(46+15 k+5 k^{2}\right)}{(5+k)^{2}(19+23 k)} i \hat{A}_{3} \hat{B}_{3}-\frac{2(7+k)}{(5+k)^{2}} i \hat{B}_{1} \hat{B}_{1}-\frac{2(7+k)}{(5+k)^{2}} i \hat{B}_{2} \hat{B}_{2} \\
& -\frac{2\left(133+361 k+40 k^{2}\right)}{(5+k)^{2}(19+23 k)} i \hat{B}_{3} \hat{B}_{3}-\frac{2\left(-41+31 k+4 k^{2}\right)}{(5+k)(19+23 k)} T^{(1)} \hat{B}_{3} \\
& \left.+\frac{2}{(5+k)} T^{(1)} \hat{A}_{3}+\frac{3(-3+k)}{(5+k)^{2}} \partial \hat{A}_{3}+\frac{2}{(5+k)} i \partial T^{(1)}\right](w)+\cdots .
\end{aligned}
$$

One can check the U(1) charge conservation in these OPEs from tables 2, 3 and 5.

As done in the OPEs between the four spin- $\frac{3}{2}$ currents and 16 higher spin currents, (6.1)-(6.4), one obtains the following results between the spin-1 currents and the 16 higher spin currents by focusing on the linear terms of higher spin currents in the right 
hand side of the OPEs

$\hat{A}_{+} \times\left(\begin{array}{cccc}T^{(1)}, & T_{+}^{\left(\frac{3}{2}\right)}, & T_{-}^{\left(\frac{3}{2}\right)}, & T^{(2)} \\ U^{\left(\frac{3}{2}\right)}, & U_{+}^{(2)}, & U_{-}^{(2)}, & U^{\left(\frac{5}{2}\right)} \\ V^{\left(\frac{3}{2}\right)}, & V_{+}^{(2)}, & V_{-}^{(2)}, & V^{\left(\frac{5}{2}\right)} \\ W^{(2)}, & W_{+}^{\left(\frac{5}{2}\right)}, & W_{-}^{\left(\frac{5}{2}\right)}, & W^{(3)}\end{array}\right) \rightarrow\left(\begin{array}{cccc}0 & U^{\left(\frac{3}{2}\right)}, & 0, & U_{-}^{(2)} \\ 0, & 0, & 0, & 0 \\ T_{-}^{\left(\frac{3}{2}\right)}, & T^{(1)}, T^{(2)}, W^{(2)}, & 0, T_{-}^{\left(\frac{3}{2}\right)}, W_{-}^{\left(\frac{5}{2}\right)} \\ U_{-}^{(2)}, & U^{\left(\frac{3}{2}\right)}, U^{\left(\frac{5}{2}\right)}, & 0, & U_{-}^{(2)}\end{array}\right)$,

$\hat{A}_{-} \times\left(\begin{array}{cccc}T^{(1)}, & T_{+}^{\left(\frac{3}{2}\right)}, & T_{-}^{\left(\frac{3}{2}\right)}, & T^{(2)} \\ U^{\left(\frac{3}{2}\right)}, & U_{+}^{(2)}, & U_{-}^{(2)}, & U^{\left(\frac{5}{2}\right)} \\ V^{\left(\frac{3}{2}\right)}, & V_{+}^{(2)}, & V_{-}^{(2)}, & V^{\left(\frac{5}{2}\right)} \\ W^{(2)}, & W_{+}^{\left(\frac{5}{2}\right)}, & W_{-}^{\left(\frac{5}{2}\right)}, & W^{(3)}\end{array}\right) \rightarrow\left(\begin{array}{cccc}0, & 0, & V^{\left(\frac{3}{2}\right)}, & V_{+}^{(2)} \\ T_{+}^{\left(\frac{3}{2}\right)}, & 0, & T^{(1)}, T^{(2)}, W^{(2)}, & T_{+}^{\left(\frac{3}{2}\right)}, W_{+}^{\left(\frac{5}{2}\right)} \\ 0, & 0, & 0, & 0 \\ V_{+}^{(2)}, & 0, & V^{\left(\frac{3}{2}\right)}, V^{\left(\frac{5}{2}\right)}, & V_{+}^{(2)}\end{array}\right)$,

$\hat{A}_{3} \times\left(\begin{array}{cccc}T^{(1)}, & T_{+}^{\left(\frac{3}{2}\right)}, & T_{-}^{\left(\frac{3}{2}\right)}, & T^{(2)} \\ U^{\left(\frac{3}{2}\right)}, & U_{+}^{(2)}, & U_{-}^{(2)}, & U^{\left(\frac{5}{2}\right)} \\ V^{\left(\frac{3}{2}\right)}, & V_{+}^{(2)}, & V_{-}^{(2)}, & V^{\left(\frac{5}{2}\right)} \\ W^{(2)}, & W_{+}^{\left(\frac{5}{2}\right)}, & W_{-}^{\left(\frac{5}{2}\right)}, & W^{(3)}\end{array}\right) \rightarrow\left(\begin{array}{cccc}0, & T_{+}^{\left(\frac{3}{2}\right)}, & T_{-}^{\left(\frac{3}{2}\right)}, & T^{(1)} \\ U^{\left(\frac{3}{2}\right)}, & 0, & U_{-}^{(2)}, & U^{\left(\frac{3}{2}\right)}, U^{\left(\frac{5}{2}\right)} \\ V^{\left(\frac{3}{2}\right)}, & V_{+}^{(2)}, & 0, & V^{\left(\frac{3}{2}\right)}, V^{\left(\frac{5}{2}\right)} \\ T^{(1)}, & T_{+}^{\left(\frac{3}{2}\right)}, W_{+}^{\left(\frac{5}{2}\right)}, & T_{-}^{\left(\frac{3}{2}\right)}, W_{-}^{\left(\frac{5}{2}\right)}, & T^{(1)}, T^{(2)}, W^{(2)}\end{array}\right)$,

$\hat{B}_{+} \times\left(\begin{array}{cccc}T^{(1)}, & T_{+}^{\left(\frac{3}{2}\right)}, & T_{-}^{\left(\frac{3}{2}\right)}, & T^{(2)} \\ U^{\left(\frac{3}{2}\right)}, & U_{+}^{(2)}, & U_{-}^{(2)}, & U^{\left(\frac{5}{2}\right)} \\ V^{\left(\frac{3}{2}\right)}, & V_{+}^{(2)}, & V_{-}^{(2)}, & V^{\left(\frac{5}{2}\right)} \\ W^{(2)}, & W_{+}^{\left(\frac{5}{2}\right)}, & W_{-}^{\left(\frac{5}{2}\right)}, & W^{(3)}\end{array}\right) \rightarrow\left(\begin{array}{cccc}0 & V^{\left(\frac{3}{2}\right)}, & 0, & V_{-}^{(2)} \\ T_{-}^{\left(\frac{3}{2}\right)}, & T^{(1)}, T^{(2)}, W^{(2)}, & 0, & T_{-}^{\left(\frac{3}{2}\right)}, W_{-}^{\left(\frac{5}{2}\right)} \\ 0, & 0, & 0, & 0 \\ V_{-}^{(2)}, & V^{\left(\frac{3}{2}\right)}, V^{\left(\frac{5}{2}\right)}, & 0, & V_{-}^{(2)}\end{array}\right)$,

$\hat{B}_{-} \times\left(\begin{array}{cccc}T^{(1)}, & T_{+}^{\left(\frac{3}{2}\right)}, & T_{-}^{\left(\frac{3}{2}\right)}, & T^{(2)} \\ U^{\left(\frac{3}{2}\right)}, & U_{+}^{(2)}, & U_{-}^{(2)}, & U^{\left(\frac{5}{2}\right)} \\ V^{\left(\frac{3}{2}\right)}, & V_{+}^{(2)}, & V_{-}^{(2)}, & V^{\left(\frac{5}{2}\right)} \\ W^{(2)}, & W_{+}^{\left(\frac{5}{2}\right)}, & W_{-}^{\left(\frac{5}{2}\right)}, & W^{(3)}\end{array}\right) \rightarrow\left(\begin{array}{cccc}0, & 0, & U^{\left(\frac{3}{2}\right)} & U_{+}^{(2)} \\ 0, & 0, & 0, & 0 \\ T_{+}^{\left(\frac{3}{2}\right)}, & 0, & T^{(1)}, T^{(2)}, W^{(2)}, T_{+}^{\left(\frac{3}{2}\right)}, W_{+}^{\left(\frac{5}{2}\right)} \\ U_{+}^{(2)}, & 0, & U^{\left(\frac{3}{2}\right)}, U^{\left(\frac{5}{2}\right)}, & U_{+}^{(2)}\end{array}\right)$,

$\hat{B}_{3} \times\left(\begin{array}{cccc}T^{(1)}, & T_{+}^{\left(\frac{3}{2}\right)}, & T_{-}^{\left(\frac{3}{2}\right)}, & T^{(2)} \\ U^{\left(\frac{3}{2}\right)}, & U_{+}^{(2)}, & U_{-}^{(2)}, & U^{\left(\frac{5}{2}\right)} \\ V^{\left(\frac{3}{2}\right)}, & V_{+}^{(2)}, & V_{-}^{(2)}, & V^{\left(\frac{5}{2}\right)} \\ W^{(2)}, & W_{+}^{\left(\frac{5}{2}\right)}, & W_{-}^{\left(\frac{5}{2}\right)}, & W^{(3)}\end{array}\right) \rightarrow\left(\begin{array}{cccc}0, & T_{+}^{\left(\frac{3}{2}\right)}, & T_{-}^{\left(\frac{3}{2}\right)}, & T^{(1)} \\ U^{\left(\frac{3}{2}\right)}, & U_{+}^{(2)}, & 0, & U^{\left(\frac{3}{2}\right)}, U^{\left(\frac{5}{2}\right)} \\ V^{\left(\frac{3}{2}\right)}, & 0, & V_{-}^{(2)}, & V^{\left(\frac{3}{2}\right)}, V^{\left(\frac{5}{2}\right)} \\ T^{(1)}, & T_{+}^{\left(\frac{3}{2}\right)}, W_{+}^{\left(\frac{5}{2}\right)}, T_{-}^{\left(\frac{3}{2}\right)}, W_{-}^{\left(\frac{5}{2}\right)}, T^{(1)}, T^{(2)}, W^{(2)}\end{array}\right)$.

Note that the zeros of the right hand sides for the OPEs between $\hat{A}_{ \pm}$and $\hat{B}_{ \pm}$acting on the 16 higher spin currents are located at the row and column containing the corresponding spin-1 current in (2.44). 


\section{B.4 The OPEs between six spin 1 currents and the higher spin currents in different basis}

In other basis described in section 6 , the OPEs between the six spin-1 currents and the four (redefined an hatted) spin- $\frac{3}{2}$ currents in (6.8) can be summarized by

$$
\begin{aligned}
\hat{A}_{ \pm}(z) \hat{T}_{ \pm}^{\left(\frac{3}{2}\right)}(w) & =\mp \frac{1}{(z-w)} i\left(\begin{array}{c}
\hat{U}^{\left(\frac{3}{2}\right)} \\
\hat{V}^{\left(\frac{3}{2}\right)}
\end{array}\right)(w)+\cdots, \\
\hat{A}_{3}(z) \hat{T}_{ \pm}^{\left(\frac{3}{2}\right)}(w) & = \pm \frac{1}{(z-w)} \frac{i}{2} \hat{T}_{ \pm}^{\left(\frac{3}{2}\right)}(w)+\cdots=\hat{B}_{3}(z) \hat{T}_{ \pm}^{\left(\frac{3}{2}\right)}(w), \\
\hat{B}_{ \pm}(z) \hat{T}_{ \pm}^{\left(\frac{3}{2}\right)}(w) & =\mp \frac{1}{(z-w)} i\left(\begin{array}{c}
\hat{V}^{\left(\frac{3}{2}\right)} \\
\hat{U}^{\left(\frac{3}{2}\right)}
\end{array}\right)(w)+\cdots, \\
\hat{A}_{\mp}(z)\left(\begin{array}{c}
\hat{U}^{\left(\frac{3}{2}\right)} \\
\hat{V}^{\left(\frac{3}{2}\right)}
\end{array}\right)(w) & =\mp \frac{1}{(z-w)} i \hat{T}_{ \pm}^{\left(\frac{3}{2}\right)}(w)+\cdots, \\
\hat{A}_{3}(z)\left(\begin{array}{c}
\hat{U}^{\left(\frac{3}{2}\right)} \\
\hat{V}^{\left(\frac{3}{2}\right)}
\end{array}\right)(w) & =\mp \frac{1}{(z-w)} \frac{i}{2}\left(\begin{array}{c}
\hat{U}^{\left(\frac{3}{2}\right)} \\
\hat{V}^{\left(\frac{3}{2}\right)}
\end{array}\right)(w)+\cdots=-\hat{B}_{3}(z)\left(\begin{array}{c}
\hat{U}^{\left(\frac{3}{2}\right)} \\
\hat{V}^{\left(\frac{3}{2}\right)}
\end{array}\right)(w), \\
\hat{B}_{ \pm}(z)\left(\begin{array}{c}
\hat{U}^{\left(\frac{3}{2}\right)} \\
\hat{V}^{\left(\frac{3}{2}\right)}
\end{array}\right)(w) & = \pm \frac{1}{(z-w)} i \hat{T}_{\mp}^{\left(\frac{3}{2}\right)}(w)+\cdots .
\end{aligned}
$$

The four spin- $\frac{3}{2}$ currents, $\hat{T}_{ \pm}^{\left(\frac{3}{2}\right)}(z), \hat{U}^{\left(\frac{3}{2}\right)}(z)$ and $\hat{V}^{\left(\frac{3}{2}\right)}(z)$, in (B.33) transform nontrivially under the two $\mathrm{SU}(2)$ currents. It is easy to see that there are no $\hat{G}_{a}(w)$-dependences in the right hand side of (B.33). Furthermore, if one writes the above four spin- $\frac{3}{2}$ currents, $\hat{U}^{\left(\frac{3}{2}\right)}(w), \hat{T}_{-}^{\left(\frac{3}{2}\right)}(w), \hat{T}_{+}^{\left(\frac{3}{2}\right)}(w)$ and $\hat{V}^{\left(\frac{3}{2}\right)}(w)$ as 11-,12-,21-,22-components of $(\mathbf{2}, \mathbf{2})$ in $\mathrm{SU}(2) \times \mathrm{SU}(2)$ respectively, then the half of (B.33) will lead to the equation appendix (A.4) where $\hat{G}_{a}(w)$ is replaced with the above four quantities and the remaining equations of appendix (B.33) become the equation appendix (A.6) with same replacement of $\hat{G}_{a}(w)$.

The OPEs between the six spin-1 currents and the six spin-2 currents in (6.8) can be described by

$$
\begin{aligned}
& \hat{A}_{+}(z)\left(\begin{array}{c}
\hat{U}_{-}^{(2)} \\
\hat{T}^{(2)}-\hat{W}^{(2)} \\
\hat{V}_{+}^{(2)}
\end{array}\right)(w)=\frac{1}{(z-w)}\left(\begin{array}{c}
0 \\
2 i \hat{U}_{-}^{(2)} \\
\left\{\hat{A}_{+} \hat{V}_{+}^{(2)}\right\}_{-1}
\end{array}\right)(w)+\cdots \\
& \hat{A}_{-}(z)\left(\begin{array}{c}
\hat{U}_{-}^{(2)} \\
\hat{T}^{(2)}-\hat{W}^{(2)} \\
\hat{V}_{+}^{(2)}
\end{array}\right)(w)=\frac{1}{(z-w)}\left(\begin{array}{c}
\left\{\hat{A}_{-} \hat{U}_{-}^{(2)}\right\}_{-1} \\
2 i \hat{V}_{+}^{(2)} \\
0
\end{array}\right)(w)+\cdots, \\
& \hat{A}_{3}(z)\left(\begin{array}{c}
\hat{U}_{-}^{(2)} \\
\hat{T}^{(2)}-\hat{W}^{(2)} \\
\hat{V}_{+}^{(2)}
\end{array}\right)(w)=\frac{1}{(z-w)} i\left(\begin{array}{c}
-\hat{U}_{-}^{(2)} \\
0 \\
\hat{V}_{+}^{(2)}
\end{array}\right)(w)+\cdots
\end{aligned}
$$




$$
\begin{aligned}
& \hat{B}_{+}(z)\left(\begin{array}{c}
\hat{V}_{-}^{(2)} \\
\hat{T}^{(2)}+\hat{W}^{(2)} \\
\hat{U}_{+}^{(2)}
\end{array}\right)(w)=\frac{1}{(z-w)}\left(\begin{array}{c}
0 \\
2 i \hat{V}_{-}^{(2)} \\
\left\{\hat{B}_{+} \hat{U}_{+}^{(2)}\right\}_{-1}
\end{array}\right)(w)+\cdots, \\
& \hat{B}_{-}(z)\left(\begin{array}{c}
\hat{V}_{-}^{(2)} \\
\hat{T}^{(2)}+\hat{W}^{(2)} \\
\hat{U}_{+}^{(2)}
\end{array}\right)(w)=\frac{1}{(z-w)}\left(\begin{array}{c}
\left\{\hat{B}_{-} \hat{V}_{-}^{(2)}\right\}_{-1} \\
2 i \hat{U}_{+}^{(2)} \\
0
\end{array}\right)(w)+\cdots, \\
& \hat{B}_{3}(z)\left(\begin{array}{c}
\hat{V}_{-}^{(2)} \\
\hat{T}^{(2)}+\hat{W}^{(2)} \\
\hat{U}_{+}^{(2)}
\end{array}\right)(w)=\frac{1}{(z-w)} i\left(\begin{array}{c}
-\hat{V}_{-}^{(2)} \\
0 \\
\hat{U}_{+}^{(2)}
\end{array}\right)(w)+\cdots
\end{aligned}
$$

The first half of (B.34) implies that the spin-2 currents, $\hat{U}_{-}^{(2)}(w),\left(\hat{T}^{(2)}-\hat{W}^{(2)}\right)(w)$ and $\hat{V}_{+}^{(2)}(w)$ transform under the SU(2) current $\hat{A}_{i}(z)$ nontrivially and the OPEs between the other $\mathrm{SU}(2)$ current $\hat{B}_{i}(z)$ and these three spin-2 currents do not contain any singular terms (i.e., singlet under the second $\mathrm{SU}(2)$ realized by $\left.\hat{B}_{i}(z)\right)$. Note that the OPE between the $\hat{A}_{+}(z)$ and the top component $\hat{U}_{-}^{(2)}(w)$ does not produce any singular term, the OPE between the $\hat{A}_{+}(z)$ and the middle component $\left(\hat{T}^{(2)}-\hat{W}^{(2)}\right)(w)$ produces the above top component $\hat{U}_{-}^{(2)}(w)$ and the OPE between the $\hat{A}_{+}(z)$ and the bottom component $\hat{V}_{+}^{(2)}(w)$ produces the above middle component $\left(\hat{T}^{(2)}-\hat{W}^{(2)}\right)(w)$ and other nonlinear terms which will be determined soon.

Similarly, the remaining equations of (B.34) imply that the spin-2 currents, $\hat{V}_{-}^{(2)}(w)$, $\left(\hat{T}^{(2)}+\hat{W}^{(2)}\right)(w)$ and $\hat{U}_{+}^{(2)}(w)$ transform under the $\mathrm{SU}(2)$ current $\hat{B}_{i}(z)$ nontrivially and the OPEs between these three spin- 2 currents and the other $\mathrm{SU}(2)$ current $\hat{A}_{i}(z)$ do not contain the singular terms (that is singlet under the first $\mathrm{SU}(2)$ realized by $\hat{A}_{i}(z)$ ). One can also analyze the raising and lowering operators of the second $\mathrm{SU}(2)$ acting on the three representations of $\mathrm{SU}(2)$ as above. In summary, the above six spin-2 currents transform as $(\mathbf{3}, \mathbf{1}) \oplus(\mathbf{1}, \mathbf{3})$ under the $\mathrm{SU}(2) \times \mathrm{SU}(2)$. All the second-order poles appeared in previous basis are disappeared in this new basis (B.34).

The nonlinear terms appearing in (B.34) are given by

$$
\begin{aligned}
& \left\{\hat{A}_{+} \hat{V}_{+}^{(2)}\right\}_{-1}(w)=\left\{\hat{A}_{-} \hat{U}_{-}^{(2)}\right\}_{-1}(w)=i\left(\hat{T}^{(2)}-\hat{W}^{(2)}\right)(w)+\frac{6 i k}{(3+7 k)} \tilde{\hat{T}}(w), \\
& \left\{\hat{B}_{+} \hat{U}_{+}^{(2)}\right\}_{-1}(w)=\left\{\hat{B}_{-} \hat{V}_{-}^{(2)}\right\}_{-1}(w)=i\left(\hat{T}^{(2)}+\hat{W}^{(2)}\right)(w)-\frac{2 i(3+4 k)}{(3+7 k)} \tilde{\hat{T}}(w),
\end{aligned}
$$

where the spin-2 primary field (under the $\hat{T}(z)$ ) is given by

$$
\tilde{\hat{T}}(w)=\left[\hat{T}-\frac{3(9 k+13)}{20(k+2)} \hat{T}^{(1)} \hat{T}^{(1)}+\frac{1}{(k+2)} \sum_{i=1}^{3} \hat{A}_{i} \hat{A}_{i}+\frac{1}{5} \sum_{i=1}^{3} \hat{B}_{i} \hat{B}_{i}\right](w) .
$$

The presence of $\hat{T}^{(1)} \hat{T}^{(1)}(w)$ makes the fourth-order pole in the OPE between $\hat{T}(z)$ and $\tilde{\hat{T}}(w)$ to vanish. The third-order pole in the OPE between $\hat{T}(z)$ and each term of (B.35) vanishes. 
Finally, the nontrivial OPEs between the six spin- 1 currents and the four spin- $\frac{5}{2}$ currents in (6.8) can be given by

$$
\begin{aligned}
\hat{A}_{\mp}(z)\left(\begin{array}{c}
\hat{U}^{\left(\frac{5}{2}\right)} \\
\hat{V}^{\left(\frac{5}{2}\right)}
\end{array}\right)(w) & =\mp \frac{1}{(z-w)} i \hat{W}_{ \pm}^{\left(\frac{5}{2}\right)}(w)+\cdots, \\
\hat{A}_{3}(z)\left(\begin{array}{c}
\hat{U}^{\left(\frac{5}{2}\right)} \\
\hat{V}^{\left(\frac{5}{2}\right)}
\end{array}\right)(w) & =\mp \frac{1}{(z-w)} \frac{i}{2}\left(\begin{array}{c}
\hat{U}^{\left(\frac{5}{2}\right)} \\
\hat{V}^{\left(\frac{5}{2}\right)}
\end{array}\right)+\cdots=-\hat{B}_{3}(z)\left(\begin{array}{c}
\hat{U}^{\left(\frac{5}{2}\right)} \\
\hat{V}^{\left(\frac{5}{2}\right)}
\end{array}\right)(w), \\
\hat{B}_{ \pm}(z)\left(\begin{array}{c}
\hat{U}^{\left(\frac{5}{2}\right)} \\
\hat{V}^{\left(\frac{5}{2}\right)}
\end{array}\right)(w) & =\mp \frac{1}{(z-w)} i \hat{W}_{\mp}^{\left(\frac{5}{2}\right)}(w)+\cdots, \\
\hat{A}_{ \pm}(z) \hat{W}_{ \pm}^{\left(\frac{5}{2}\right)}(w) & =\mp \frac{1}{(z-w)} i\left(\begin{array}{c}
\hat{U}^{\left(\frac{5}{2}\right)} \\
\hat{V}^{\left(\frac{5}{2}\right)}
\end{array}\right)(w)+\cdots, \\
\hat{A}_{3}(z) \hat{W}_{ \pm}^{\left(\frac{5}{2}\right)}(w) & = \pm \frac{1}{(z-w)} \frac{i}{2} \hat{W}_{ \pm}^{\left(\frac{5}{2}\right)}(w)+\cdots=\hat{B}_{3}(z) \hat{W}_{ \pm}^{\left(\frac{5}{2}\right)}(w) \\
\hat{B}_{ \pm}(z) \hat{W}_{ \pm}^{\left(\frac{5}{2}\right)}(w) & = \pm \frac{1}{(z-w)} i\left(\begin{array}{c}
\hat{V}^{\left(\frac{5}{2}\right)} \\
\hat{U}^{\left(\frac{5}{2}\right)}
\end{array}\right)(w)+\cdots .
\end{aligned}
$$

The four spin- $\frac{5}{2}$ currents, $\hat{W}_{ \pm}^{\left(\frac{5}{2}\right)}(z), \hat{U}^{\left(\frac{5}{2}\right)}(z)$ and $\hat{V}^{\left(\frac{5}{2}\right)}(z)$, in (B.36) transform nontrivially under the two $\mathrm{SU}(2)$ currents as in (B.33). There are sign changes in the OPEs between $\hat{B}_{ \pm}(z)$ and these spin- $\frac{5}{2}$ currents. All the second-order poles appeared in previous basis are disappeared in this new basis (B.36).

Note that the OPEs between the six spin- 1 currents and the spin-1 current $\hat{T}^{(1)}(w)=$ $T^{(1)}(w)$ do not have any singular terms and similarly the OPEs between those six spin-1 currents and the spin-3 current $\hat{W}^{(3)}(w)$ do not contain any singular terms. This reflects the fact that these two higher spin currents transform as $(\mathbf{1}, \mathbf{1})$ under the $\mathrm{SU}(2) \times \mathrm{SU}(2)$ respectively. In other words, $\hat{A}_{i}(z) \hat{T}^{(1)}(w)=\hat{B}_{i}(z) \hat{T}^{(1)}(w)=\hat{A}_{i}(z) \hat{W}^{(3)}(w)=\hat{B}_{i}(z) \hat{W}^{(3)}(w)=$ $+\cdots$. The OPEs between the four spin- $\frac{3}{2}$ currents $\hat{G}_{a}(z)$ and the 16 higher spin currents in this new basis will be given at the end of appendix $C$.

\section{The OPEs between the large $\mathcal{N}=4$ nonlinear algebra currents and the higher spin currents-II}

In this appendix, we present the OPEs between the four spin- $\frac{3}{2}$ currents in section 3 and the 16 higher spin currents in section 4.

\section{C.1 The OPEs between four spin $\frac{3}{2}$ currents and the higher spin current of spins $\left(1, \frac{3}{2}, \frac{3}{2}, 2\right)$}

From the explicit expressions (3.14)-(3.17) and (4.7), the following OPEs between the spin- $\frac{3}{2}$ currents and the spin- 1 current can be derived

$$
\left(\begin{array}{l}
\hat{G}_{11} \\
\hat{G}_{22}
\end{array}\right)(z) T^{(1)}(w)=\frac{1}{(z-w)}\left(\begin{array}{c}
\hat{G}_{11}+2 U^{\left(\frac{3}{2}\right)} \\
-\hat{G}_{22}+2 V^{\left(\frac{3}{2}\right)}
\end{array}\right)(w)+\cdots,
$$




$$
\left(\begin{array}{c}
\hat{G}_{12} \\
\hat{G}_{21}
\end{array}\right)(z) T^{(1)}(w)=\frac{1}{(z-w)}\left(\begin{array}{c}
-\hat{G}_{12}+2 T_{-}^{\left(\frac{3}{2}\right)} \\
\hat{G}_{21}+2 T_{+}^{\left(\frac{3}{2}\right)}
\end{array}\right)(w)+\cdots
$$

One can check the U(1) charge conservation in these OPEs from table 2 .

For the spin- $\frac{3}{2}$ currents (4.10) and (4.14), one obtains the following OPEs between the spin- $\frac{3}{2}$ currents and the spin- $\frac{3}{2}$ currents as follows:

$$
\begin{aligned}
\left(\begin{array}{c}
\hat{G}_{11} \\
\hat{G}_{22}
\end{array}\right)(z) T_{ \pm}^{\left(\frac{3}{2}\right)}(w)= & \frac{1}{(z-w)^{2}} \frac{2 k}{(5+k)}\left[i \hat{B}_{\mp}\right](w) \\
& +\frac{1}{(z-w)}\left[-\left(\begin{array}{c}
U_{+}^{(2)} \\
V_{-}^{(2)}
\end{array}\right)+\frac{1}{(5+k)}\left(\mp 4 \hat{A}_{3} \hat{B}_{\mp}+k i \partial \hat{B}_{\mp}\right)\right](w)+\cdots, \\
\left(\begin{array}{l}
\hat{G}_{12} \\
\hat{G}_{21}
\end{array}\right)(z) T_{ \pm}^{\left(\frac{3}{2}\right)}(w)= & \mp \frac{1}{(z-w)^{3}} \frac{6 k}{(5+k)}+\frac{1}{(z-w)^{2}}\left[\frac{2 i}{(5+k)}\left(-3 \hat{A}_{3}-k \hat{B}_{3}\right)+T^{(1)}\right](w) \\
& +\frac{1}{(z-w)}\left[\mp \frac{6 k}{(3+7 k)} \hat{T} \mp T^{(2)}+\frac{i}{(5+k)}\left(-3 \partial \hat{A}_{3}-k \partial \hat{B}_{3}\right)\right. \\
& \left.+\frac{1}{2} \partial T^{(1)}\right](w)+\cdots, \\
\left(\begin{array}{l}
\hat{G}_{21} \\
\hat{G}_{12}
\end{array}\right)(z) T_{ \pm}^{\left(\frac{3}{2}\right)}(w)= & \frac{1}{(z-w)} \frac{2}{(5+k)}\left[\mp \hat{A}_{\mp} \hat{B}_{\mp}\right](w)+\cdots, \\
\left(\begin{array}{l}
\hat{G}_{22} \\
\hat{G}_{11}
\end{array}\right)(z) T_{ \pm}^{\left(\frac{3}{2}\right)}(w)= & \frac{1}{(z-w)^{2}} \frac{6}{(5+k)}\left[i \hat{A}_{\mp}\right](w) \\
& +\frac{1}{(z-w)}\left[-\left(\begin{array}{l}
V_{+}^{(2)} \\
U_{-}^{(2)}
\end{array}\right)+\frac{3}{(5+k)} i \partial \hat{A}_{\mp}\right](w)+\cdots
\end{aligned}
$$

The U(1) charge conservation in these OPEs can be checked from tables 2 and 3 .

For the last component spin-2 current, one calculates the following OPEs between the spin- $\frac{3}{2}$ currents and the spin-2 current as follows:

$$
\begin{aligned}
& \hat{G}_{11}(z) T^{(2)}(w)=\frac{1}{(z-w)^{2}}\left[\frac{\left(12-11 k+5 k^{2}\right)}{(3+7 k)(5+k)} \hat{G}_{11}+\frac{(-3+k)}{(5+k)} U^{\left(\frac{3}{2}\right)}\right](w) \\
& +\frac{1}{(z-w)}\left[U^{\left(\frac{5}{2}\right)}+\frac{k(-39+5 k)}{(9+21 k)(5+k)} \partial \hat{G}_{11}+\frac{(-7+k)}{3(5+k)} \partial U^{\left(\frac{3}{2}\right)}\right. \\
& \left.+\frac{2}{(5+k)}\left(2 i \hat{A}_{3}\left(\hat{G}_{11}+U^{\left(\frac{3}{2}\right)}\right)-i \hat{B}_{-} T_{-}^{\left(\frac{3}{2}\right)}\right)\right](w)+\cdots, \\
& \left(\begin{array}{l}
\hat{G}_{12} \\
\hat{G}_{21}
\end{array}\right)(z) T^{(2)}(w)=\frac{1}{(z-w)^{2}}\left[-\frac{\left(-3+38 k+9 k^{2}\right)}{(3+7 k)(5+k)}\left(\begin{array}{c}
\hat{G}_{12} \\
\hat{G}_{21}
\end{array}\right) \pm \frac{(11+3 k)}{(5+k)} T_{\mp}^{\left(\frac{3}{2}\right)}\right](w) \\
& +\frac{1}{(z-w)}\left[-\frac{\left(3+22 k+3 k^{2}\right)}{(3+7 k)(5+k)} \partial\left(\begin{array}{c}
\hat{G}_{12} \\
\hat{G}_{21}
\end{array}\right) \pm \partial T_{\mp}^{\left(\frac{3}{2}\right)}\right.
\end{aligned}
$$




$$
\begin{aligned}
& \left.+\frac{2}{(5+k)}\left(\mp i \hat{A}_{ \pm}\left(\begin{array}{c}
\hat{G}_{22} \\
\hat{G}_{11}
\end{array}\right)+i \hat{A}_{ \pm}\left(\begin{array}{c}
V^{\left(\frac{3}{2}\right)} \\
U^{\left(\frac{3}{2}\right)}
\end{array}\right)+i \hat{B}_{ \pm}\left(\begin{array}{c}
U^{\left(\frac{3}{2}\right)} \\
V^{\left(\frac{3}{2}\right)}
\end{array}\right)\right)\right](w) \\
& +\cdots, \\
\hat{G}_{22}(z) T^{(2)}(w)= & \frac{1}{(z-w)^{2}}\left[\frac{\left(12-11 k+5 k^{2}\right)}{(3+7 k)(5+k)} \hat{G}_{22}-\frac{(-3+k)}{(5+k)} V^{\left(\frac{3}{2}\right)}\right](w) \\
& +\frac{1}{(z-w)}\left[V^{\left(\frac{5}{2}\right)}+\frac{k(-39+5 k)}{(9+21 k)(5+k)} \partial \hat{G}_{22}-\frac{(1+k)}{3(5+k)} \partial V^{\left(\frac{3}{2}\right)}\right. \\
& \left.+\frac{2}{(5+k)}\left(-i \hat{A} T_{-}^{\left(\frac{3}{2}\right)}-2 i \hat{A}_{3} \hat{G}_{22}+i \hat{B}_{+} \hat{G}_{21}+2 i \hat{B}_{3} V^{\left(\frac{3}{2}\right)}\right)\right](w)+\cdots .
\end{aligned}
$$

The U(1) charge conservation in these OPEs can be checked from tables 2, 3 and 4 .

\section{C.2 The OPEs between four spin $\frac{3}{2}$ currents and two higher spin currents of spins $\left(\frac{3}{2}, 2,2, \frac{5}{2}\right)$}

From (3.14)-(3.17), (4.21) and (4.35), the following OPEs between the spin- $\frac{3}{2}$ currents and the spin- $\frac{3}{2}$ currents can be obtained

$$
\begin{aligned}
\left(\begin{array}{l}
\hat{G}_{11} \\
\hat{G}_{22}
\end{array}\right)(z)\left(\begin{array}{l}
U^{\left(\frac{3}{2}\right)} \\
V^{\left(\frac{3}{2}\right)}
\end{array}\right)(w)= & \frac{1}{(z-w)} \frac{2}{(5+k)}\left[ \pm \hat{A}_{ \pm} \hat{B}_{\mp}\right](w)+\cdots, \\
\left(\begin{array}{l}
\hat{G}_{12} \\
\hat{G}_{21}
\end{array}\right)(z)\left(\begin{array}{l}
U^{\left(\frac{3}{2}\right)} \\
V^{\left(\frac{3}{2}\right)}
\end{array}\right)(w)= & \frac{1}{(z-w)^{2}} \frac{6}{(5+k)}\left[i \hat{A}_{ \pm}\right](w) \\
& +\frac{1}{(z-w)}\left[\left(\begin{array}{l}
U_{-}^{(2)} \\
V_{+}^{(2)}
\end{array}\right)+\frac{3}{(5+k)} i \partial \hat{A}_{ \pm}\right](w)+\cdots, \\
\left(\begin{array}{l}
\hat{G}_{21} \\
\hat{G}_{12}
\end{array}\right)(z)\left(\begin{array}{l}
U^{\left(\frac{3}{2}\right)} \\
V^{\left(\frac{3}{2}\right)}
\end{array}\right)(w)= & \frac{1}{(z-w)^{2}} \frac{2 k}{(5+k)}\left[-i \hat{B}_{\mp}\right](w) \\
& +\frac{1}{(z-w)}\left[\left(\begin{array}{l}
U_{+}^{(2)} \\
V_{-}^{(2)}
\end{array}\right)-\frac{k}{(5+k)} i \partial \hat{B}_{\mp}\right](w)+\cdots, \\
\left(\begin{array}{l}
\hat{G}_{22} \\
\hat{G}_{11}
\end{array}\right)(z)\left(\begin{array}{l}
U^{\left(\frac{3}{2}\right)} \\
V^{\left(\frac{3}{2}\right)}
\end{array}\right)(w)= & \mp \frac{1}{(z-w)^{3}} \frac{6 k}{(5+k)} \\
& +\frac{1}{(z-w)^{2}}\left[\frac{2 i}{(5+k)}\left(3 \hat{A}_{3}-k \hat{B}_{3}\right)+T^{(1)}\right](w) \\
& +\frac{1}{(z-w)}\left[\mp W^{(2)}+\frac{3 i}{(5+k)} \partial \hat{A}_{3}-\frac{i k}{(5+k)} \partial \hat{B}_{3}+\frac{1}{2} \partial T^{(1)}\right](w) \\
& +\cdots \cdot(\mathrm{C} .13)
\end{aligned}
$$

The U(1) charge conservation in these OPEs can be checked from tables 2 and 3.

For the spin-2 currents (4.24) and (4.42), one calculates the nontrivial OPEs between the spin- $\frac{3}{2}$ currents and the spin- 2 currents

$$
\left(\begin{array}{c}
\hat{G}_{11} \\
\hat{G}_{22}
\end{array}\right)(z)\left(\begin{array}{c}
U_{+}^{(2)} \\
V_{-}^{(2)}
\end{array}\right)(w)=-\frac{1}{(z-w)} \frac{2}{(5+k)}\left[i \hat{B}_{\mp}\left(\begin{array}{c}
\hat{G}_{11}+U^{\left(\frac{3}{2}\right)} \\
\hat{G}_{22}-V^{\left(\frac{3}{2}\right)}
\end{array}\right)\right](w)+\cdots,
$$




$$
\begin{aligned}
\hat{G}_{12}(z) U_{+}^{(2)}(w)= & \frac{1}{(z-w)^{2}}\left[\frac{(6+k)}{(5+k)} \hat{G}_{11}+\frac{2(7+k)}{(5+k)} U^{\left(\frac{3}{2}\right)}\right](w) \\
& +\frac{1}{(z-w)}\left[-U^{\left(\frac{5}{2}\right)}+\frac{(6+k)}{3(5+k)} \partial \hat{G}_{11}+\frac{2(11+k)}{3(5+k)} \partial U^{\left(\frac{3}{2}\right)}\right. \\
& +\frac{2}{(5+k)}\left(-i \hat{A}_{+} T_{+}^{\left(\frac{3}{2}\right)}-2 i \hat{A}_{3} U^{\left(\frac{3}{2}\right)}-i \hat{B}_{-}\left(\hat{G}_{12}-T_{-}^{\left(\frac{3}{2}\right)}\right)\right. \\
& \left.\left.+2 i \hat{B}_{3} U^{\left(\frac{3}{2}\right)}\right)\right](w)+\cdots, \\
\left(\begin{array}{l}
\hat{G}_{21} \\
\hat{G}_{12}
\end{array}\right)(z)\left(\begin{array}{l}
U_{+}^{(2)} \\
V_{-}^{(2)}
\end{array}\right)(w)= & \mp \frac{1}{(z-w)} \frac{2}{(5+k)}\left[i \hat{B}_{\mp} T_{ \pm}^{\left(\frac{3}{2}\right)}\right](w)+\cdots, \\
\left(\begin{array}{l}
\hat{G}_{22} \\
\hat{G}_{11}
\end{array}\right)(z)\left(\begin{array}{l}
U_{+}^{(2)} \\
V_{-}^{(2)}
\end{array}\right)(w)= & \frac{1}{(z-w)^{2}}\left[\mp \frac{(8+k)}{(5+k)}\left(\begin{array}{l}
\hat{G}_{21} \\
\hat{G}_{12}
\end{array}\right)-\frac{2(7+k)}{(5+k)} T_{ \pm}^{\left(\frac{3}{2}\right)}\right](w) \\
& +\frac{1}{(z-w)}\left[ \pm W_{ \pm}^{\left(\frac{5}{2}\right)} \mp \frac{(8+k)}{3(5+k)} \partial\left(\begin{array}{l}
\hat{G}_{21} \\
\hat{G}_{12}
\end{array}\right)-\frac{2(9+k)}{3(5+k)} \partial T_{ \pm}^{\left(\frac{3}{2}\right)}\right. \\
& \left.+\frac{2}{(5+k)}\left( \pm i \hat{A}_{\mp}\left(\begin{array}{l}
U^{\left(\frac{3}{2}\right)} \\
V^{\left(\frac{3}{2}\right)}
\end{array}\right) \mp 2 i \hat{B}_{3} T_{ \pm}^{\left(\frac{3}{2}\right)}\right)\right](w)+\cdots . \quad(\mathrm{C} .1
\end{aligned}
$$

It is easy to check that the spin- $\frac{5}{2}$ fields in (C.15) and (C.17) after subtracting the descendant fields (the derivative terms for the fields in the second-order pole with coefficient $\frac{1}{3}$ ) in the first-order pole are primary under the stress energy tensor (3.2). The U(1) charge conservation in these OPEs can be checked from tables 2 and 4.

Similarly, from the expression (4.28) and (4.38), the following remaining OPEs can be described as

$$
\begin{aligned}
\left(\begin{array}{c}
\hat{G}_{11} \\
\hat{G}_{22}
\end{array}\right)(z)\left(\begin{array}{c}
U_{-}^{(2)} \\
V_{+}^{(2)}
\end{array}\right)(w)= & \frac{1}{(z-w)} \frac{2}{(5+k)}\left[i \hat{A}_{ \pm}\left(\begin{array}{c}
\hat{G}_{11}+U^{\left(\frac{3}{2}\right)} \\
\hat{G}_{22}-V^{\left(\frac{3}{2}\right)}
\end{array}\right)\right](w)+\cdots, \quad(\mathrm{C} .18) \\
\left(\begin{array}{c}
\hat{G}_{12} \\
\hat{G}_{21}
\end{array}\right)(z)\left(\begin{array}{c}
U_{-}^{(2)} \\
V_{+}^{(2)}
\end{array}\right)(w)= & -\frac{1}{(z-w)} \frac{2}{(5+k)}\left[i \hat{A}_{ \pm}\left(\begin{array}{c}
\hat{G}_{12}-T_{-}^{\left(\frac{3}{2}\right)} \\
\hat{G}_{21}+T_{+}^{\left(\frac{3}{2}\right)}
\end{array}\right)\right](w)+\cdots, \quad(\mathrm{C} .19) \\
\hat{G}_{21}(z) U_{-}^{(2)}(w)= & \frac{1}{(z-w)^{2}}\left[\frac{(3+2 k)}{(5+k)} \hat{G}_{11}+\frac{4(2+k)}{(5+k)} U^{\left(\frac{3}{2}\right)}\right](w) \quad(\mathrm{C} .20) \\
& +\frac{1}{(z-w)}\left[U^{\left(\frac{5}{2}\right)}+\frac{(3+2 k)}{3(5+k)} \partial \hat{G}_{11}+\frac{4(2+k)}{3(5+k)} \partial U^{\left(\frac{3}{2}\right)}\right](w)+\cdots, \\
\left(\begin{array}{c}
\hat{G}_{22} \\
\hat{G}_{11}
\end{array}\right)(z)\left(\begin{array}{l}
U_{-}^{(2)} \\
V_{+}^{(2)}
\end{array}\right)(w)= & \frac{1}{(z-w)^{2}}\left[ \pm \frac{(3+2 k)}{(5+k)}\left(\begin{array}{l}
\hat{G}_{12} \\
\hat{G}_{21}
\end{array}\right)-\frac{4(2+k)}{(5+k)} T_{\mp}^{\left(\frac{3}{2}\right)}\right](w) \\
& +\frac{1}{(z-w)}\left[ \pm W_{\mp}^{\left(\frac{5}{2}\right)} \pm \frac{(7+2 k)}{3(5+k)} \partial\left(\begin{array}{l}
\hat{G}_{12} \\
\hat{G}_{21}
\end{array}\right)-\frac{4(3+k)}{3(5+k)} \partial T_{\mp}^{\left(\frac{3}{2}\right)}\right. \\
& \left.+\frac{2}{(5+k)}\left(-2 i \hat{A}_{3}\left(\begin{array}{l}
\hat{G}_{12} \\
\hat{G}_{21}
\end{array}\right) \pm 2 i \hat{A}_{3} T_{\mp}^{\left(\frac{3}{2}\right)} \mp i \hat{B}_{ \pm}\left(\begin{array}{l}
U^{\left(\frac{3}{2}\right)} \\
V^{\left(\frac{3}{2}\right)}
\end{array}\right)\right)\right](w) \\
& +\cdots \cdot
\end{aligned}
$$


As above, the spin- $\frac{5}{2}$ fields in (C.21) after subtracting these descendant fields (the derivative terms for the fields in the second-order pole with coefficient $\frac{1}{3}$ ) in the first-order pole are primary. The U(1) charge conservation in these OPEs can be checked from tables 2 and 4.

For the last current of spin- $\frac{5}{2}$ (4.32), one also has the OPEs between the spin- $\frac{3}{2}$ currents and the spin- $\frac{5}{2}$ current

$$
\begin{aligned}
& \hat{G}_{11}(z) U^{\left(\frac{5}{2}\right)}(w)=\frac{1}{(z-w)^{2}} \frac{4(5+3 k)}{3(5+k)^{2}}\left[\hat{A}_{+} \hat{B}_{-}\right](w) \\
& +\frac{1}{(z-w)}\left[-\frac{2 i}{(5+k)} \hat{A}_{+} U_{+}^{(2)}+\frac{2(-4+k)}{(5+k)^{2}} \hat{A}_{+} \partial \hat{B}_{-}\right. \\
& \left.-\frac{4 i}{(5+k)} \hat{B}_{-} U_{-}^{(2)}-\frac{4}{3(5+k)} \hat{G}_{11} \hat{G}_{11}+\frac{2}{(5+k)} \hat{G}_{11} U^{\left(\frac{3}{2}\right)}\right](w)+\cdots \text {, } \\
& \hat{G}_{12}(z) U^{\left(\frac{5}{2}\right)}(w)=\frac{1}{(z-w)^{3}} \frac{4(1+3 k)}{(5+k)^{2}}\left[i \hat{A}_{+}\right](w) \\
& +\frac{1}{(z-w)^{2}}\left[\frac{2(29+4 k)}{3(5+k)} U_{-}^{(2)}-\frac{12}{(5+k)^{2}} \hat{A}_{+} \hat{A}_{3}\right. \\
& \left.+\frac{4(12+k)}{3(5+k)^{2}} \hat{A}_{+} \hat{B}_{3}+\frac{6 i}{(5+k)^{2}} \partial \hat{A}_{+}+\frac{2 i}{(5+k)} T^{(1)} \hat{A}_{+}\right](w) \\
& +\frac{1}{(z-w)}\left[\frac{4}{(5+k)} i \hat{A}_{+} \hat{T}-\frac{2}{(5+k)} i \hat{A}_{+} W^{(2)}-\frac{10}{(5+k)^{2}} \hat{A}_{+} \partial \hat{A}_{3}\right. \\
& -\frac{2(-18+k)}{3(5+k)^{2}} \hat{A}_{+} \partial \hat{B}_{3}-\frac{4}{(5+k)} i \hat{A}_{3} U_{-}^{(2)}-\frac{8}{(5+k)^{2}} \hat{A}_{3} \partial \hat{A}_{+} \\
& +\frac{4(-3+k)}{3(5+k)^{2}} \hat{B}_{3} \partial \hat{A}_{+}+\frac{2(11+k)}{3(5+k)} \partial U_{-}^{(2)}-\frac{1}{(5+k)} i \partial T^{(1)} \hat{A}_{+} \\
& -\frac{2}{(5+k)} \hat{G}_{11} \hat{G}_{12}+\frac{2}{(5+k)} \hat{G}_{11} T_{-}^{\left(\frac{3}{2}\right)}+\frac{4}{(5+k)^{2}} i \hat{A}_{+} \hat{A}_{+} \hat{A}_{-} \\
& -\frac{4}{(5+k)^{2}} i \hat{A}_{+} \hat{A}_{3} \hat{A}_{3}-\frac{8}{(5+k)^{2}} i \hat{A}_{+} \hat{A}_{3} \hat{B}_{3}+\frac{4}{(5+k)^{2}} i \hat{A}_{+} \hat{B}_{+} \hat{B}_{-} \\
& +\frac{4}{(5+k)^{2}} i \hat{A}_{+} \hat{B}_{3} \hat{B}_{3}+\frac{8}{(5+k)^{2}} i \hat{A}_{3} \hat{A}_{+} \hat{A}_{3}+\frac{2}{(5+k)} T^{(1)} \hat{A}_{+} \hat{A}_{3} \\
& \left.-\frac{2}{(5+k)} \hat{A}_{3} T^{(1)} \hat{A}_{+}+\frac{4}{(5+k)} i \hat{B}_{3} U_{-}^{(2)}\right](w)+\cdots, \\
& \hat{G}_{21}(z) U^{\left(\frac{5}{2}\right)}(w)=-\frac{1}{(z-w)^{3}} \frac{16 k}{3(5+k)^{2}}\left[i \hat{B}_{-}\right](w) \\
& +\frac{1}{(z-w)^{2}}\left[-\frac{16(2+k)}{3(5+k)} U_{+}^{(2)}-\frac{32(3+k)}{3(5+k)^{2}} \hat{A}_{3} \hat{B}_{-}\right. \\
& \left.+\frac{8 k}{(5+k)^{2}} \hat{B}_{-} \hat{B}_{3}+\frac{4 k}{(5+k)^{2}} i \partial \hat{B}_{-}+\frac{4}{(5+k)} T^{(1)} i \hat{B}_{-}\right](w) \\
& +\frac{1}{(z-w)}\left[-\frac{4(3+2 k)}{3(5+k)^{2}} \hat{A}_{3} \partial \hat{B}_{-}+\frac{12 k}{(3+7 k)(5+k)} i \hat{B}_{-} \hat{T}\right. \\
& -\frac{2}{(5+k)} i \hat{B}_{-} W^{(2)}-\frac{4(2+k)}{3(5+k)} \partial U_{+}^{(2)}+\frac{2 k}{3(5+k)^{2}} i \partial^{2} \hat{B}_{-} \\
& +\frac{2(9+2 k)}{3(5+k)^{2}} i \hat{A}_{-} \hat{A}_{+} \hat{B}_{-}+\frac{4 k}{(5+k)^{2}} i \hat{B}_{-} \hat{B}_{3} \hat{B}_{3}-\frac{4 k}{(5+k)^{2}} i \hat{B}_{3} \hat{B}_{-} \hat{B}_{3}
\end{aligned}
$$




$$
\begin{aligned}
& -\frac{2}{(5+k)} T^{(1)} \hat{B}_{-} \hat{B}_{3}+\frac{2}{(5+k)} \hat{B}_{3} T^{(1)} \hat{B}_{-}-\frac{2(3+2 k)}{3(5+k)^{2}} i \hat{A}_{+} \hat{A}_{-} \hat{B}_{-} \\
& \left.+\frac{2}{(5+k)} i \hat{B}_{-} T^{(2)}\right](w)+\cdots \\
& \hat{G}_{22}(z) U^{\left(\frac{5}{2}\right)}(w)=\frac{1}{(z-w)^{3}}\left[\frac{16}{(5+k)^{2}} i\left(\hat{A}_{3}-4 i \hat{B}_{3}\right)-\frac{4(-5+2 k)}{3(5+k)} T^{(1)}\right](w) \\
& +\frac{1}{(z-w)^{2}}\left[-\frac{4\left(18-9 k+10 k^{2}\right)}{3(3+7 k)(5+k)} \hat{T}+\frac{2(7+2 k)}{(5+k)} T^{(2)}\right. \\
& +\frac{4(3-4 k)}{3(5+k)^{2}} \hat{A}_{1} \hat{A}_{1}+\frac{4(3-4 k)}{3(5+k)^{2}} \hat{A}_{2} \hat{A}_{2}-\frac{8(3+2 k)}{3(5+k)^{2}} \hat{A}_{3} \hat{A}_{3} \\
& +\frac{8(3+2 k)}{3(5+k)^{2}} \hat{A}_{3} \hat{B}_{3}-\frac{8(3+2 k)}{3(5+k)^{2}} \hat{B}_{1} \hat{B}_{1}-\frac{8(3+2 k)}{3(5+k)^{2}} \hat{B}_{2} \hat{B}_{2} \\
& \left.+\frac{8(-3+k)}{3(5+k)^{2}} \hat{B}_{3} \hat{B}_{3}+\frac{4 i}{(5+k)} T^{(1)} \hat{B}_{3}+\frac{2(-5+2 k)}{3(5+k)} W^{(2)}\right](w) \\
& +\frac{1}{(z-w)}\left[-\frac{1}{2} W^{(3)}+\frac{1}{2} P^{(3)}-\frac{8(-3+k)}{(19+23 k)} T^{(1)} \hat{T}+\frac{1}{(5+k)} i \hat{A}_{+} V_{+}^{(2)}\right. \\
& +\frac{1}{(5+k)} i \hat{A}_{-} U_{-}^{(2)}+\frac{2(9-4 k))}{3(5+k)^{2}} \hat{A}_{-} \partial \hat{A}_{+}-\frac{3 k}{(5+k)^{2}} \hat{B}_{-} \partial \hat{B}_{+} \\
& +\frac{8\left(48+97 k+29 k^{2}\right)}{(3+7 k)(19+23 k)(5+k)} i \hat{A}_{3} \hat{T}-\frac{4}{(5+k)} i \hat{A}_{3} T^{(2)}+\frac{4(9+k)}{3(5+k)^{2}} \hat{A}_{3} \partial \hat{B}_{3} \\
& +\frac{1}{(5+k)} i \hat{B}_{+} U_{+}^{(2)}+\frac{k}{3(5+k)^{2}} \hat{B}_{+} \partial \hat{B}_{-}-\frac{1}{(5+k)} i \hat{B}_{-} V_{-}^{(2)} \\
& -\frac{8(-27+k) k}{(3+7 k)(19+23 k)(5+k)} i \hat{B}_{3} \hat{T}+\frac{4}{(5+k)} i \hat{B}_{3} T^{(2)}+\frac{4(-6+k)}{3(5+k)^{2}} \hat{B}_{3} \partial \hat{A}_{3} \\
& -\frac{4}{(5+k)} i \hat{B}_{3} W^{(2)}-\frac{2 k(-39+5 k)}{3(3+7 k)(5+k)} \partial \hat{T}+\partial T^{(2)}+\frac{(-7+k)}{3(5+k)} \partial W^{(2)} \\
& +\frac{(9+4 k)}{3(5+k)^{2}} i \partial^{2} \hat{A}_{3}+\frac{2 k}{(5+k)^{2}} i \partial^{2} \hat{B}_{3}+\frac{2}{3(5+k)} \partial^{2} T^{(1)}-\frac{2}{(5+k)} i \partial T^{(1)} \hat{A}_{3} \\
& +\frac{2}{(5+k)} i T^{(1)} \partial \hat{A}_{3}+\frac{2}{(5+k)} i T^{(1)} \partial \hat{B}_{3}-\frac{2}{(5+k)} \hat{G}_{12} \hat{G}_{21} \\
& -\frac{2}{(5+k)} \hat{G}_{22} U^{\left(\frac{3}{2}\right)}+\frac{(15-8 k)}{3(5+k)^{2}} i \hat{A}_{+} \hat{A}_{-} \hat{A}_{3}+\frac{4(3+k)}{3(5+k)^{2}} i \hat{A}_{-} \hat{A}_{+} \hat{A}_{3} \\
& +\frac{(-3+4 k)}{3(5+k)^{2}} i \hat{A}_{3} \hat{A}_{+} \hat{A}_{-}+\frac{8}{(5+k)^{2}} i \hat{A}_{3} \hat{A}_{3} \hat{A}_{3}-\frac{16}{(5+k)^{2}} i \hat{A}_{3} \hat{A}_{3} \hat{B}_{3} \\
& +\frac{8}{(5+k)^{2}} i \hat{A}_{3} \hat{B}_{3} \hat{B}_{3}+\frac{8}{(5+k)^{2}} i \hat{B}_{-} \hat{A}_{3} \hat{B}_{+}-\frac{2 k}{3(5+k)^{2}} i \hat{B}_{-} \hat{B}_{+} \hat{B}_{3} \\
& \left.+\frac{2 k}{3(5+k)^{2}} i \hat{B}_{3} \hat{B}_{+} \hat{B}_{-}-\frac{2}{(5+k)} \hat{G}_{21} T_{-}^{\left(\frac{3}{2}\right)}\right](w)+\cdots,
\end{aligned}
$$

where the $W^{(3)}(w)$ is given by (4.60) and the other spin-3 field which is primary can be written in terms of known currents as follows:

$$
\begin{aligned}
P^{(3)}(w)= & -\frac{2 i}{(5+k)}\left[-\frac{i}{2}(5+k) W^{(3)}+\hat{A}_{+} V_{+}^{(2)}-\frac{i}{(5+k)} \hat{A}_{+} \partial \hat{A}_{-}\right. \\
& +\hat{A}_{-} U_{-}^{(2)}-\frac{i}{(5+k)} \hat{A}_{-} \partial \hat{A}_{+}-\frac{2 i}{(5+k)} \hat{A}_{3} \partial \hat{A}_{3}+\frac{2 i(-2+k)}{(5+k)} \hat{A}_{3} \partial \hat{B}_{3}
\end{aligned}
$$




$$
\begin{aligned}
& -\hat{B}_{+} U_{+}^{(2)}-\frac{i}{(5+k)} \hat{B}_{+} \partial \hat{B}_{-}-\hat{B}_{-} V_{-}^{(2)} \\
& -\frac{i}{(5+k)} \hat{B}_{-} \partial \hat{B}_{+}-\frac{2 i(-4+k)}{(5+k)} \hat{B}_{3} \partial \hat{A}_{3}-\frac{2 i}{(5+k)} \hat{B}_{3} \partial \hat{B}_{3}-i \partial \hat{T}+i \partial W^{(2)} \\
& \left.-\partial T^{(1)} \hat{A}_{3}+\partial T^{(1)} \hat{B}_{3}-T^{(1)} \partial \hat{B}_{3}+\frac{i}{2} T^{(1)} \hat{A}_{+} \hat{A}_{-}-\frac{i}{2} \hat{A}_{-} T^{(1)} \hat{A}_{+}\right](w) .(\mathrm{C} .
\end{aligned}
$$

The U(1) charge conservation in these OPEs can be checked from tables 2, 3 and 2 .

For the spin-2 current (4.38), one obtains

$$
\begin{aligned}
\hat{G}_{12}(z) V_{+}^{(2)}(w)= & \frac{1}{(z-w)^{2}}\left[-\frac{(3+2 k)}{(5+k)} \hat{G}_{22}+\frac{4(2+k)}{(5+k)} V^{\left(\frac{3}{2}\right)}\right](w) \\
& +\frac{1}{(z-w)}\left[-V^{\left(\frac{5}{2}\right)}-\frac{(3+2 k)}{3(5+k)} \partial \hat{G}_{22}+\frac{4(4+k)}{3(5+k)} \partial V^{\left(\frac{3}{2}\right)}\right. \\
& \left.+\frac{2}{(5+k)}\left(i \hat{A}_{-} T_{-}^{\left(\frac{3}{2}\right)}+2 i \hat{A}_{3} V^{\left(\frac{3}{2}\right)}-i \hat{B}_{+}\left(\hat{G}_{21}+T_{+}^{\left(\frac{3}{2}\right)}\right)-2 i \hat{B}_{3} V^{\left(\frac{3}{2}\right)}\right)\right](w) \\
& +\cdots
\end{aligned}
$$

As above, the spin- $\frac{5}{2}$ fields after subtracting the descendant fields (the derivative terms for the fields in the second-order pole with coefficient $\frac{1}{3}$ ) in the first-order pole are primary. The U(1) charge conservation in these OPEs can be checked from tables 2 and 4 .

For the other spin-2 current (4.42), the following OPEs can be described as

$$
\begin{aligned}
\hat{G}_{21}(z) V_{-}^{(2)}(w)= & \frac{1}{(z-w)^{2}}\left[-\frac{(6+k)}{(5+k)} \hat{G}_{22}+\frac{2(7+k)}{(5+k)} V^{\left(\frac{3}{2}\right)}\right](w) \\
& +\frac{1}{(z-w)}\left[V^{\left(\frac{5}{2}\right)}-\frac{(6+k)}{3(5+k)} \partial \hat{G}_{22}+\frac{2(7+k)}{3(5+k)} \partial V^{\left(\frac{3}{2}\right)}\right](w)+\cdots
\end{aligned}
$$

As above, the spin- $\frac{5}{2}$ fields after subtracting these descendant fields (the derivative terms for the fields in the second-order pole with coefficient $\frac{1}{3}$ ) in the first-order pole are primary. The U(1) charge conservation in these OPEs can be checked from tables 2 and 4 .

For the spin- $\frac{5}{2}$ current (4.46), one has the following nontrivial OPEs between the spin- $\frac{3}{2}$ currents and the spin- $\frac{5}{2}$ current

$$
\begin{aligned}
\hat{G}_{11}(z) V^{\left(\frac{5}{2}\right)}(w)= & \frac{1}{(z-w)^{3}}\left[\frac{8 i(-1+3 k)}{(5+k)^{2}} \hat{A}_{3}-\frac{16 i k}{3(5+k)^{2}} \hat{B}_{3}+\frac{4(-7+2 k)}{3(5+k)} T^{(1)}\right](w) \\
& +\frac{1}{(z-w)^{2}}\left[-\frac{4\left(27+12 k+10 k^{2}\right)}{(9+21 k)(5+k)} \hat{T}+\frac{2(7+2 k)}{(5+k)} T^{(2)}+\frac{2(-7+2 k)}{3(5+k)} W^{(2)}\right. \\
& -\frac{4(9+4 k)}{3(5+k)^{2}} \hat{A}_{1} \hat{A}_{1}-\frac{4(9+4 k)}{3(5+k)^{2}} \hat{A}_{2} \hat{A}_{2}-\frac{4(27+4 k)}{3(5+k)^{2}} \hat{A}_{3} \hat{A}_{3}+\frac{40(3+k)}{3(5+k)^{2}} \hat{A}_{3} \hat{B}_{3} \\
& \left.-\frac{4(9+7 k)}{3(5+k)^{2}} \hat{B}_{1} \hat{B}_{1}-\frac{4(9+7 k)}{3(5+k)^{2}} \hat{B}_{2} \hat{B}_{2}-\frac{4(9+4 k)}{3(5+k)^{2}} \hat{B}_{3} \hat{B}_{3}+\frac{4 i}{(5+k)} T^{(1)} \hat{A}_{3}\right](w) \\
& +\frac{1}{(z-w)}\left[\frac{3}{2} W^{(3)}+\frac{1}{2} P^{(3)}+\frac{8(-3+k)}{(19+23 k)} T^{(1)} \hat{T}+\frac{3}{(5+k)} i \hat{A}_{+} V_{+}^{(2)}\right. \\
& -\frac{(3+4 k)}{3(5+k)^{2}} \hat{A}_{+} \partial \hat{A}_{-}+\frac{1}{(5+k)} i \hat{A}_{-} U_{-}^{(2)}-\frac{(9+4 k)}{3(5+k)^{2}} \hat{A}_{-} \partial \hat{A}_{+} \\
& -\frac{8\left(48+97 k+29 k^{2}\right)}{(3+7 k)(19+23 k)(5+k)} \hat{A}_{3} \hat{T}+\frac{4}{(5+k)} i \hat{A}_{3} T^{(2)}+\frac{4}{(5+k)} i \hat{A}_{3} W^{(2)}
\end{aligned}
$$




$$
\begin{aligned}
& -\frac{8(3+k)}{3(5+k)^{2}} \hat{A}_{3} \partial \hat{A}_{3}-\frac{1}{(5+k)} i \hat{B}_{+} U_{+}^{(2)}-\frac{(12+17 k)}{3(5+k)^{2}} \hat{B}_{+} \partial \hat{B}_{-} \\
& +\frac{k}{(5+k)^{2}} \hat{B}_{-} \partial \hat{B}_{+}+\frac{8(-27+k) k}{(3+7 k)(19+23 k)(5+k)} i \hat{B}_{3} \hat{T} \\
& -\frac{4}{(5+k)} i \hat{B}_{3} T^{(2)}+\frac{4(-3+4 k)}{3(5+k)^{2}} \hat{B}_{3} \partial \hat{A}_{3}-\frac{2\left(9-18 k+5 k^{2}\right)}{3(3+7 k)(5+k)} \partial \hat{T}+\partial T^{(2)} \\
& +\frac{(-5+k)}{3(5+k)} \partial W^{(2)}-\frac{2(3+8 k)}{3(5+k)^{2}} i \partial^{2} \hat{B}_{3}+\frac{2}{3(5+k)} \partial^{2} T^{(1)} \\
& +\frac{4}{(5+k)} i T^{(1)} \partial \hat{A}_{3}-\frac{2}{(5+k)} \hat{G}_{11} V^{\left(\frac{3}{2}\right)}-\frac{2}{(5+k)} \hat{G}_{21} T_{-}^{\left(\frac{3}{2}\right)} \\
& -\frac{4}{(5+k)^{2}} i \hat{A}_{+} \hat{A}_{-} \hat{A}_{3}-\frac{4}{(5+k)} i \hat{A}_{3} \hat{A}_{+} \hat{A}_{-}-\frac{8}{(5+k)^{2}} i \hat{A}_{3} \hat{A}_{3} \hat{A}_{3} \\
& +\frac{16}{(5+k)^{2}} i \hat{A}_{3} \hat{A}_{3} \hat{B}_{3}+\frac{2(9+k)}{3(5+k)^{2}} i \hat{A}_{3} \hat{B}_{+} \hat{B}_{-}-\frac{8}{(5+k)^{2}} i \hat{A}_{3} \hat{B}_{3} \hat{B}_{3} \\
& -\frac{2(21+k)}{3(5+k)^{2}} i \hat{B}_{-} \hat{A}_{3} \hat{B}_{+}+\frac{2(3+2 k)}{3(5+k)^{2}} i \hat{B}_{-} \hat{B}_{+} \hat{B}_{3}-\frac{2(3+2 k)}{3(5+k)^{2}} i \hat{B}_{3} \hat{B}_{+} \hat{B}_{-} \\
& \left.-\frac{1}{(5+k)} i \hat{B}_{-} V_{-}^{(2)}-\frac{2}{(5+k)} i \partial T^{(1)} \hat{A}_{3}\right](w)+\cdots \\
\hat{G}_{12}(z) V^{\left(\frac{5}{2}\right)}(w)= & -\frac{1}{(z-w)^{3}} \frac{40 k}{3(5+k)^{2}}\left[i \hat{B}_{+}\right](w)
\end{aligned}
$$$$
+\frac{1}{(z-w)^{2}}\left[\frac{2(17+8 k)}{3(5+k)} V_{-}^{(2)}-\frac{4(9+2 k)}{3(5+k)^{2}} \hat{A}_{3} \hat{B}_{+}\right.
$$$$
\left.+\frac{4 k}{(5+k)^{2}} \hat{B}_{+} \hat{B}_{3}-\frac{2 k}{(5+k)^{2}} i \partial \hat{B}_{+}+\frac{2}{(5+k)^{2}} T^{(1)} i \hat{B}_{+}\right](w)
$$$$
+\frac{1}{(z-w)}\left[\frac{4}{(5+k)} i \hat{A}_{3} V_{-}^{(2)}+\frac{4(-9+k)}{3(5+k)^{2}} \hat{A}_{3} \partial \hat{B}_{+}-\frac{4}{(5+k)} i \hat{B}_{+} \hat{T}\right.
$$$$
+\frac{2}{(5+k)} i \hat{B}_{+} W^{(2)}-\frac{4}{(5+k)} i \hat{B}_{3} V_{-}^{(2)}+\frac{4(4+k)}{3(5+k)} \partial V_{-}^{(2)}
$$$$
-\frac{(-6+5 k)}{6(5+k)^{2}} i \partial^{2} \hat{B}_{+}-\frac{1}{(5+k)} i \partial T^{(1)} \hat{B}_{+}+\frac{2}{(5+k)} i T^{(1)} \partial \hat{B}_{+}
$$$$
+\frac{2}{(5+k)} \hat{G}_{22} T_{-}^{\left(\frac{3}{2}\right)}-\frac{(15+4 k)}{3(5+k)^{2}} i \hat{A}_{+} \hat{A}_{-} \hat{B}_{+}+\frac{(3+4 k)}{3(5+k)^{2}} i \hat{A}_{-} \hat{A}_{+} \hat{B}_{+}
$$$$
-\frac{4}{(5+k)^{2}} i \hat{A}_{3} \hat{A}_{3} \hat{B}_{+}+\frac{(-6+k)}{2(5+k)^{2}} i \hat{B}_{+} \hat{B}_{+} \hat{B}_{-}-\frac{4}{(5+k)^{2}} i \hat{B}_{+} \hat{B}_{3} \hat{B}_{3}
$$

$$
\begin{aligned}
& \left.-\frac{(2+k)}{2(5+k)^{2}} i \hat{B}_{-} \hat{B}_{+} \hat{B}_{+}+\frac{8}{(5+k)^{2}} i \hat{B}_{3} \hat{A}_{3} \hat{B}_{+}\right](w)+\cdots, \\
\hat{G}_{21}(z) V^{\left(\frac{5}{2}\right)}(w)= & \frac{1}{(z-w)^{3}} \frac{16 k}{(5+k)^{2}}\left[i \hat{A}_{-}\right](w)
\end{aligned}
$$

$$
\begin{aligned}
& +\frac{1}{(z-w)^{2}}\left[-\frac{8(7+k)}{3(5+k)} V_{+}^{(2)}-\frac{24}{(5+k)^{2}} \hat{A}_{-} \hat{A}_{3}\right. \\
& \left.+\frac{16(9+k)}{3(5+k)^{2}} \hat{A}_{-} \hat{B}_{3}-\frac{12}{(5+k)^{2}} i \partial \hat{A}_{-}+\frac{4}{(5+k)} T^{(1)} i \hat{A}_{-}\right](w) \\
& +\frac{1}{(z-w)}\left[-\frac{4(3+4 k)}{(3+7 k)(5+k)} i \hat{A}_{-} \hat{T}+\frac{2}{(5+k)} i \hat{A}_{-} T^{(2)}\right. \\
& +\frac{12}{(5+k)^{2}} \hat{A}_{-} \partial \hat{A}_{3}+\frac{4(15+k)}{3(5+k)^{2}} \hat{A}_{-} \partial \hat{B}_{3}+\frac{4}{(5+k)^{2}} \hat{A}_{3} \partial \hat{A}_{-}
\end{aligned}
$$




$$
\begin{aligned}
& -\frac{2(7+k)}{3(5+k)} \partial V_{+}^{(2)}+\frac{2}{(5+k)} i T^{(1)} \partial \hat{A}_{-}-\frac{4}{(5+k)^{2}} i \hat{A}_{+} \hat{A}_{-} \hat{A}_{-} \\
& +\frac{4(12+k)}{3(5+k)^{2}} i \hat{A}_{-} \hat{A}_{3} \hat{B}_{3}-\frac{8}{(5+k)^{2}} i \hat{A}_{-} \hat{B}_{+} \hat{B}_{-}-\frac{4}{(5+k)^{2}} i \hat{A}_{-} \hat{B}_{3} \hat{B}_{3} \\
& +\frac{16}{(5+k)^{2}} i \hat{A}_{3} \hat{A}_{-} \hat{A}_{3}-\frac{4(6+k)}{3(5+k)^{2}} i \hat{A}_{3} \hat{A}_{-} \hat{B}_{3}-\frac{20}{(5+k)^{2}} i \hat{A}_{-} \hat{A}_{3} \hat{A}_{3} \\
& \left.+\frac{2}{(5+k)} i \hat{A}_{-} W^{(2)}\right](w)+\cdots \\
\hat{G}_{22}(z) V^{\left(\frac{5}{2}\right)}(w)= & \frac{1}{(z-w)^{2}} \frac{56}{3(5+k)^{2}}\left[-\hat{A}_{-} \hat{B}_{+}\right](w) \\
& +\frac{1}{(z-w)}\left[-\frac{4}{(5+k)} i \hat{A}_{-} V_{-}^{(2)}-\frac{2}{(5+k)} i \hat{B}_{+} V_{+}^{(2)}\right. \\
& \left.+\frac{2}{(5+k)^{2}} \hat{B}_{+} \partial \hat{A}_{-}+\frac{4}{3(5+k)} \hat{G}_{22} \hat{G}_{22}+\frac{2}{(5+k)} \hat{G}_{22} V^{\left(\frac{3}{2}\right)}\right](w)+\cdots,
\end{aligned}
$$

where $P_{3}(w)$ is given by appendix (C.26). The spin-3 field for the first three OPEs after subtracting the descendant fields (the derivative terms for the fields in the second-order pole with coefficient $\frac{1}{4}$ ) in the first-order pole are quasi-primary and those for the last OPE is primary. The $\mathrm{U}(1)$ charge conservation in these OPEs can be checked from tables 2, 3 and 5 .

\section{C.3 The OPEs between four spin $\frac{3}{2}$ currents and the higher spin current of spins $\left(2, \frac{5}{2}, \frac{5}{2}, 3\right)$}

From the explicit expressions (3.14)-(3.17), and (4.49), the following four OPEs between the spin- $\frac{3}{2}$ currents and the spin- 2 current can be obtained

$$
\begin{aligned}
\left(\begin{array}{c}
\hat{G}_{11} \\
\hat{G}_{22}
\end{array}\right)(z) W^{(2)}(w)= & \frac{1}{(z-w)^{2}} \frac{1}{(5+k)}\left[\left(\begin{array}{c}
\hat{G}_{11} \\
\hat{G}_{22}
\end{array}\right)+(11+3 k)\left(\begin{array}{c}
-U^{\left(\frac{3}{2}\right)} \\
V^{\left(\frac{3}{2}\right)}
\end{array}\right)\right](w) \\
& +\frac{1}{(z-w)} \frac{1}{(5+k)}\left[-\partial\left(\begin{array}{c}
\hat{G}_{11} \\
\hat{G}_{22}
\end{array}\right)+(5+k) \partial\left(\begin{array}{c}
-U^{\left(\frac{3}{2}\right)} \\
V^{\left(\frac{3}{2}\right)}
\end{array}\right)\right. \\
\pm & \left.2 i \hat{A}_{ \pm}\left(\begin{array}{c}
\hat{G}_{21} \\
\hat{G}_{12}
\end{array}\right)+2 i \hat{A}_{ \pm} T_{ \pm}^{\left(\frac{3}{2}\right)}-2 i \hat{B}_{\mp} T_{\mp}^{\left(\frac{3}{2}\right)}\right](w)+\cdots, \\
\left(\begin{array}{c}
\hat{G}_{12} \\
\hat{G}_{21}
\end{array}\right)(z) W^{(2)}(w)= & \frac{1}{(z-w)^{2}} \frac{1}{(5+k)}\left[2(2+k)\left(\begin{array}{c}
\hat{G}_{12} \\
\hat{G}_{21}
\end{array}\right) \mp(-3+k) T_{\mp}^{\left(\frac{3}{2}\right)}\right](w) \\
& +\frac{1}{(z-w)} \frac{1}{(5+k)}\left[W_{\mp}^{\left(\frac{5}{2}\right)}+\frac{1}{3} 2(2+k) \partial\left(\begin{array}{c}
\hat{G}_{12} \\
\hat{G}_{21}
\end{array}\right)\right. \\
\mp & \left.\frac{1}{3}(-3+k) \partial T_{\mp}^{\left(\frac{3}{2}\right)}\right](w)+\cdots .
\end{aligned}
$$

As above, the spin- $\frac{5}{2}$ fields after subtracting the descendant fields (the derivative terms for the fields in the second-order pole with coefficient $\frac{1}{3}$ ) in the first-order pole are primary. The U(1) charge conservation in these OPEs can be checked from tables 2 and 4 . 
For the spin- $\frac{5}{2}$ current (4.53), one has the following OPEs between the spin- $\frac{3}{2}$ currents and the spin- $\frac{5}{2}$ current

$$
\begin{aligned}
& \hat{G}_{11}(z) W_{+}^{\left(\frac{5}{2}\right)}(w)=\frac{1}{(z-w)^{3}} \frac{56 k}{3(5+k)^{2}}\left[i \hat{B}_{-}\right](w) \\
& +\frac{1}{(z-w)^{2}}\left[\frac{2(15+8 k)}{3(5+k)} U_{+}^{(2)}+\frac{4(-1+2 k)}{3(5+k)^{2}} \hat{A}_{3} \hat{B}_{-}\right. \\
& \left.-\frac{4 k}{(5+k)^{2}} \hat{B}_{-} \hat{B}_{3}-\frac{2 k}{(5+k)^{2}} i \partial \hat{B}_{-}-\frac{2}{(5+k)} T^{(1)} i \hat{B}_{-}\right](w) \\
& +\frac{1}{(z-w)}\left[\frac{4}{(5+k)} i \hat{A}_{3} U_{+}^{(2)}+\frac{12 k}{(3+7 k)(5+k)} i \hat{B}_{-} \hat{T}+\frac{2}{(5+k)} i \hat{B}_{-} T^{(2)}\right. \\
& +\frac{2(1+4 k)}{3(5+k)^{2}} \hat{B}_{-} \partial \hat{A}_{3}+\frac{4(3+k)}{3(5+k)} \partial U_{+}^{(2)}-\frac{k}{3(5+k)^{2}} i \partial^{2} \hat{B}_{-} \\
& +\frac{1}{(5+k)} i \partial T^{(1)} \hat{B}_{-}+\frac{2}{(5+k)} \hat{G}_{11} \hat{G}_{21}+\frac{2}{(5+k)} \hat{G}_{11} T_{+}^{\left(\frac{3}{2}\right)} \\
& -\frac{4}{(5+k)^{2}} i \hat{A}_{+} \hat{A}_{-} \hat{B}_{-}-\frac{4(-5+k)}{3(5+k)^{2}} i \hat{A}_{3} \hat{B}_{-} \hat{B}_{3}+\frac{k}{(5+k)^{2}} i \hat{B}_{+} \hat{B}_{-} \hat{B}_{-} \\
& -\frac{k}{(5+k)^{2}} i \hat{B}_{-} \hat{B}_{+} \hat{B}_{-}-\frac{4 k}{(5+k)^{2}} i \hat{B}_{-} \hat{B}_{3} \hat{B}_{3}+\frac{4(-5+k)}{3(5+k)^{2}} i \hat{B}_{3} \hat{A}_{3} \hat{B}_{-} \\
& \left.+\frac{4 k}{(5+k)^{2}} i \hat{B}_{3} \hat{B}_{-} \hat{B}_{3}+\frac{2}{(5+k)} T^{(1)} \hat{B}_{-} \hat{B}_{3}-\frac{2}{(5+k)} \hat{B}_{3} T^{(1)} \hat{B}_{-}\right](w)+\cdots, \\
& \hat{G}_{12}(z) W_{+}^{\left(\frac{5}{2}\right)}(w)=\frac{1}{(z-w)^{3}}\left[-\frac{8 i(1+3 k)}{(5+k)^{2}} \hat{A}_{3}-\frac{80 i k}{3(5+k)^{2}} \hat{B}_{3}-\frac{8(-3+k)}{3(5+k)} T^{(1)}\right](w) \\
& +\frac{1}{(z-w)^{2}}\left[-\frac{4\left(15+65 k+22 k^{2}\right)}{3(3+7 k)(5+k)} \hat{T}+\frac{4(-3+k)}{3(5+k)} T^{(2)}+\frac{4(4+k)}{(5+k)} W^{(2)}\right. \\
& -\frac{16(-1+k)}{3(5+k)^{2}} \hat{A}_{1} \hat{A}_{1}-\frac{16(-1+k)}{3(5+k)^{2}} \hat{A}_{2} \hat{A}_{2}+\frac{4(13-4 k)}{3(5+k)^{2}} \hat{A}_{3} \hat{A}_{3} \\
& -\frac{4}{3(5+k)} \hat{B}_{1} \hat{B}_{1}-\frac{4}{3(5+k)} \hat{B}_{2} \hat{B}_{2}+\frac{4(-5+2 k)}{3(5+k)^{2}} \hat{B}_{3} \hat{B}_{3}-\frac{4 i}{(5+k)} T^{(1)} \hat{A}_{3} \\
& \left.+\frac{4 i}{(5+k)} T^{(1)} \hat{B}_{3}+\frac{8(-4+k)}{3(5+k)^{2}} \hat{A}_{3} \hat{B}_{3}\right](w) \\
& +\frac{1}{(z-w)}\left[\frac{1}{4} \partial\left\{\hat{G}_{12} W_{+}^{\left(\frac{5}{2}\right)}\right\}_{-2}+\frac{24 i(1+3 k)}{(19+23 k)(5+k)}\left(\hat{T} \hat{A}_{3}-\frac{1}{2} \partial^{2} \hat{A}_{3}\right)\right. \\
& +\frac{80 i k}{(19+23 k)(5+k)}\left(\hat{T} \hat{B}_{3}-\frac{1}{2} \partial^{2} \hat{B}_{3}\right) \\
& \left.+P^{(3)}+\frac{8(-3+k)}{(19+23 k)}\left(\hat{T} T^{(1)}-\frac{1}{2} \partial^{2} T^{(1)}\right)\right](w)+\cdots, \\
& \hat{G}_{21}(z) W_{+}^{\left(\frac{5}{2}\right)}(w)=\frac{1}{(z-w)^{2}} \frac{4(19+3 k)}{3(5+k)^{2}}\left[-\hat{A}_{-} \hat{B}_{-}\right](w) \\
& +\frac{1}{(z-w)}\left[-\frac{11}{3(5+k)} \hat{G}_{21} \hat{G}_{21}+\frac{2}{(5+k)} i \hat{A}_{-} U_{+}^{(2)}\right. \\
& \left.-\frac{2(-3+k)}{(5+k)^{2}} \hat{A}_{-} \partial \hat{B}_{-}-\frac{2}{(5+k)} i \hat{B}_{-} V_{+}^{(2)}\right](w)+\cdots, \\
& \hat{G}_{22}(z) W_{+}^{\left(\frac{5}{2}\right)}(w)=\frac{1}{(z-w)^{3}} \frac{4(5+3 k)}{(5+k)^{2}}\left[i \hat{A}_{-}\right](w) \\
& +\frac{1}{(z-w)^{2}}\left[-\frac{2(27+4 k)}{3(5+k)} V_{+}^{(2)}-\frac{12}{(5+k)^{2}} \hat{A}_{-} \hat{A}_{3}\right.
\end{aligned}
$$




$$
\begin{aligned}
& \left.+\frac{4(2+k)}{3(5+k)^{2}} \hat{A}_{-} \hat{B}_{3}-\frac{6}{(5+k)^{2}} i \partial \hat{A}_{-}+\frac{2}{(5+k)} T^{(1)} i \hat{A}_{-}\right](w) \\
& +\frac{1}{(z-w)}\left[\frac{4(3+4 k)}{(3+7 k)(5+k)} i \hat{A}_{-} \hat{T}_{-} \frac{2}{(5+k)} i \hat{A}_{-} T^{(2)}\right. \\
& -\frac{2(-10+k)}{3(5+k)^{2}} \hat{A}_{-} \partial \hat{B}_{3}-\frac{4}{(5+k)^{2}} \hat{A}_{3} \partial \hat{A}_{-}-\frac{4}{(5+k)} i \hat{B}_{3} V_{+}^{(2)} \\
& -\frac{1}{(5+k)} i \partial T^{(1)} \hat{A}_{-}+\frac{2}{(5+k)} i T^{(1)} \partial \hat{A}_{-}-\frac{2}{(5+k)} \hat{G}_{22} T_{+}^{\left(\frac{3}{2}\right)} \\
& +\frac{4}{(5+k)^{2}} i \hat{A}_{+} \hat{A}_{-} \hat{A}_{-}-\frac{4}{(5+k)^{2}} i \hat{A}_{-} \hat{A}_{3} \hat{A}_{3}+\frac{4(-7+k)}{3(5+k)^{2}} i \hat{A}_{-} \hat{A}_{3} \hat{B}_{3} \\
& +\frac{4}{(5+k)^{2}} i \hat{A}_{-} \hat{B}_{3} \hat{B}_{3}+\frac{8}{(5+k)^{2}} i \hat{A}_{3} \hat{A}_{-} \hat{A}_{3}-\frac{4(-1+k)}{3(5+k)^{2}} i \hat{A}_{3} \hat{A}_{-} \hat{B}_{3} \\
& \left.-\frac{2(9+k)}{3(5+k)} \partial V_{+}^{(2)}-\frac{6}{(5+k)^{2}} \hat{A}_{-} \partial \hat{A}_{3}\right](w)+\cdots,
\end{aligned}
$$

where $P_{3}(w)$ is given by appendix (C.26). The spin-3 field for the third OPE after subtracting the descendant fields (the derivative terms for the fields in the second-order pole with coefficient $\frac{1}{4}$ ) in the first-order pole is primary and those for the remaining OPEs are quasi-primary. The $\mathrm{U}(1)$ charge conservation in these OPEs can be checked from tables 2,3 and 5 .

For the other spin- $\frac{5}{2}$ current (4.56), the following OPEs between the spin- $\frac{3}{2}$ currents and the spin- $\frac{5}{2}$ current can be obtained

$$
\begin{aligned}
\hat{G}_{11}(z) W_{-}^{\left(\frac{5}{2}\right)}(w)= & -\frac{1}{(z-w)^{3}} \frac{4(5+3 k)}{(5+k)^{2}}\left[i \hat{A}_{+}\right](w) \\
& +\frac{1}{(z-w)^{2}}\left[\frac{2(27+4 k)}{3(5+k)} U_{-}^{(2)}-\frac{12}{(5+k)^{2}} \hat{A}_{+} \hat{A}_{3}\right. \\
& \left.+\frac{4(2+k)}{3(5+k)^{2}} \hat{A}_{+} \hat{B}_{3}+\frac{6}{(5+k)^{2}} i \partial \hat{A}_{+}+\frac{2}{(5+k)} T^{(1)} i \hat{A}_{+}\right](w) \\
& +\frac{1}{(z-w)}\left[-\frac{4(3+4 k)}{(3+7 k)(5+k)} i \hat{A}_{+} \hat{T}+\frac{2}{(5+k)} i \hat{A}_{+} T^{(2)}\right. \\
& -\frac{2(-10+k)}{3(5+k)^{2}} \hat{A}_{+} \partial \hat{B}_{3}+\frac{4}{(5+k)^{2}} \hat{A}_{3} \partial \hat{A}_{+}-\frac{4}{(5+k)} i \hat{B}_{3} U_{-}^{(2)} \\
& -\frac{1}{(5+k)} i \partial T^{(1)} \hat{A}_{+}+\frac{2}{(5+k)} i T^{(1)} \partial \hat{A}_{+}+\frac{2}{(5+k)} \hat{G}_{11} T_{-}^{\left(\frac{3}{2}\right)} \\
& +\frac{12}{(5+k)^{2}} i \hat{A}_{+} \hat{A}_{3} \hat{A}_{3}-\frac{4(-7+k)}{3(5+k)^{2}} i \hat{A}_{+} \hat{A}_{3} \hat{B}_{3}-\frac{4}{(5+k)^{2}} i \hat{A}_{+} \hat{B}_{3} \hat{B}_{3} \\
& -\frac{16}{(5+k)^{2}} i \hat{A}_{3} \hat{A}_{+} \hat{A}_{3}+\frac{4(-1+k)}{3(5+k)^{2}} i \hat{A}_{3} \hat{A}_{+} \hat{B}_{3}-\frac{4}{(5+k)^{2}} i \hat{A}_{+} \hat{A}_{+} \hat{A}_{-} \\
& \left.+\frac{10}{(5+k)^{2}} \hat{A}_{+} \partial \hat{A}_{3}+\frac{2(9+k)}{3(5+k)} \partial U_{-}^{(2)}\right](w)+\cdots, \\
\hat{G}_{12}(z) W_{-}^{\left(\frac{5}{2}\right)}(w)= & \frac{1}{(z-w)^{2}} \frac{4(19+3 k)}{3(5+k)^{2}}\left[-\hat{A}_{+} \hat{B}_{+}\right](w)
\end{aligned}
$$




$$
\begin{aligned}
& +\frac{1}{(z-w)}\left[-\frac{11}{3(5+k)} \hat{G}_{12} \hat{G}_{12}-\frac{2}{(5+k)} i \hat{B}_{+} U_{-}^{(2)}\right. \\
& \left.+\frac{2}{(5+k)} i \hat{A}_{+} V_{-}^{(2)}-\frac{2(-3+k)}{(5+k)^{2}} \hat{A}_{+} \partial \hat{B}_{+}\right](w)+\cdots, \\
& \hat{G}_{21}(z) W_{-}^{\left(\frac{5}{2}\right)}(w)=\frac{1}{(z-w)^{3}}\left[\frac{8 i(1+3 k)}{(5+k)^{2}} \hat{A}_{3}+\frac{80 i k}{3(5+k)^{2}} \hat{B}_{3}+\frac{8(-3+k)}{3(5+k)} T^{(1)}\right](w) \\
& +\frac{1}{(z-w)^{2}}\left[-\frac{4\left(15+65 k+22 k^{2}\right)}{3(3+7 k)(5+k)} \hat{T}+\frac{4(-3+k)}{3(5+k)} T^{(2)}+\frac{4(4+k)}{(5+k)} W^{(2)}\right. \\
& -\frac{16(-1+k)}{3(5+k)^{2}} \hat{A}_{1} \hat{A}_{1}-\frac{16(-1+k)}{3(5+k)^{2}} \hat{A}_{2} \hat{A}_{2}+\frac{4(13-4 k)}{3(5+k)^{2}} \hat{A}_{3} \hat{A}_{3} \\
& -\frac{4}{3(5+k)} \hat{B}_{1} \hat{B}_{1}-\frac{4}{3(5+k)} \hat{B}_{2} \hat{B}_{2}+\frac{4(-5+2 k)}{3(5+k)^{2}} \hat{B}_{3} \hat{B}_{3} \\
& \left.-\frac{4 i}{(5+k)} T^{(1)} \hat{A}_{3}+\frac{4 i}{(5+k)} T^{(1)} \hat{B}_{3}+\frac{8(-4+k)}{3(5+k)^{2}} \hat{A}_{3} \hat{B}_{3}\right](w) \\
& +\frac{1}{(z-w)}\left[\frac{1}{4} \partial\left\{\hat{G}_{21} W_{-}^{\left(\frac{5}{2}\right)}\right\}_{-2}+\frac{24 i(1+3 k)}{(19+23 k)(5+k)}\left(\hat{T} \hat{A}_{3}-\frac{1}{2} \partial^{2} \hat{A}_{3}\right)\right. \\
& +\frac{80 i k}{(19+23 k)(5+k)}\left(\hat{T} \hat{B}_{3}-\frac{1}{2} \partial^{2} \hat{B}_{3}\right) \\
& \left.+W^{(3)}+\frac{8(-3+k)}{(19+23 k)}\left(\hat{T} T^{(1)}-\frac{1}{2} \partial^{2} T^{(1)}\right)\right](w)+\cdots,
\end{aligned}
$$


Finally, from the explicit for the spin-3 current (4.60), the following complicated OPEs between the spin- $\frac{3}{2}$ currents and the spin- 3 current can be obtained

$$
\begin{aligned}
& \hat{G}_{11}(z) W^{(3)}(w)=\frac{1}{(z-w)^{3}}\left[-\frac{2(-3+k)\left(571+357 k+110 k^{2}\right)}{3(5+k)^{2}(19+23 k)} \hat{G}_{11}\right. \\
& \left.-\frac{2(-3+k)\left(1489+1225 k+220 k^{2}\right)}{3(5+k)^{2}(19+23 k)} U^{\left(\frac{3}{2}\right)}\right](w) \\
& +\frac{1}{(z-w)^{2}}\left[-\frac{(19+5 k)}{(5+k)} U^{\left(\frac{5}{2}\right)}+\frac{2(-10+k)}{(5+k)^{2}} i \hat{A}_{+} \hat{G}_{21}\right. \\
& -\frac{2(14+3 k)}{(5+k)^{2}} i \hat{A}_{+} T_{+}^{\left(\frac{3}{2}\right)}-\frac{2\left(-5+211 k+100 k^{2}\right)}{(5+k)^{2}(19+23 k)} i \hat{A}_{3} \hat{G}_{11} \\
& -\frac{6(7+3 k)}{(5+k)^{2}} i \hat{A}_{3} U^{\left(\frac{3}{2}\right)}-\frac{(-5+7 k)}{(5+k)^{2}} i \hat{B}_{-} \hat{G}_{12}-\frac{18}{(5+k)^{2}} i \hat{B}_{-} T_{-}^{\left(\frac{3}{2}\right)} \\
& -\frac{2\left(76+411 k+83 k^{2}\right)}{(5+k)(19+23 k)} i \hat{B}_{3} \hat{G}_{11}+\frac{2(-1+3 k)}{(5+k)^{2}} i \hat{B}_{3} U^{\left(\frac{3}{2}\right)} \\
& -\frac{\left(-1047+16 k+251 k^{2}+12 k^{3}\right)}{3(5+k)^{2}(19+23 k)} \partial \hat{G}_{11} \\
& \left.-\frac{4\left(-640-355 k-73 k^{2}+6 k^{3}\right)}{3(5+k)^{2}(19+23 k)} \partial U^{\left(\frac{3}{2}\right)}-\frac{\left(-199+k+12 k^{2}\right)}{(5+k)(19+23 k)} T^{(1)} \hat{G}_{11}\right](w) \\
& +\frac{1}{(z-w)}\left[\frac{4}{(5+k)^{2}} \hat{A}_{-} \hat{A}_{+} U^{\left(\frac{3}{2}\right)}+\frac{4}{(5+k)^{2}} \hat{B}_{-} \hat{A}_{+} V^{\left(\frac{3}{2}\right)}-\frac{2}{(5+k)} i \hat{B}_{-} W_{-}^{\left(\frac{5}{2}\right)}\right. \\
& +\frac{8}{(5+k)^{2}} \hat{B}_{-} \hat{A}_{3} \hat{G}_{12}-\frac{8}{(5+k)^{2}} \hat{B}_{-} \hat{A}_{3} T_{-}^{\left(\frac{3}{2}\right)}-\frac{1}{(5+k)} \hat{B}_{-} \hat{B}_{+} U^{\left(\frac{3}{2}\right)} \\
& +\frac{2(7+2 k)}{(5+k)^{2}} \hat{B}_{-} \hat{B}_{3} T_{-}^{\left(\frac{3}{2}\right)}-\frac{1}{3(5+k)} i \hat{B}_{-} \partial \hat{G}_{12}+\frac{2(9+4 k)}{3(5+k)^{2}} i \hat{B}_{-} \partial T_{-}^{\left(\frac{3}{2}\right)} \\
& -\frac{4}{(5+k)^{2}} \hat{A}_{+} \hat{A}_{-} \hat{G}_{11}+\frac{1}{(5+k)} \hat{B}_{+} \hat{B}_{-} U^{\left(\frac{3}{2}\right)}-\frac{8}{(5+k)^{2}} \hat{A}_{+} \hat{A}_{3} \hat{G}_{21} \\
& +\frac{2(4+k)}{(5+k)^{2}} i \hat{A}_{+} \partial \hat{G}_{21}+\frac{2(6+k)}{(5+k)^{2}} i \hat{A}_{+} \partial T_{+}^{\left(\frac{3}{2}\right)}-\frac{4}{(5+k)} i \hat{A}_{3} U^{\left(\frac{5}{2}\right)} \\
& -\frac{4}{(5+k)^{2}} \hat{A}_{3} \hat{A}_{3} \hat{G}_{11}+\frac{16}{(5+k)^{2}} \hat{A}_{3} \hat{A}_{3} U^{\left(\frac{3}{2}\right)}+\frac{8}{(5+k)^{2}} \hat{A}_{3} \hat{B}_{3} \hat{G}_{11} \\
& -\frac{16}{(5+k)^{2}} \hat{A}_{3} \hat{B}_{3} U^{\left(\frac{3}{2}\right)}-\frac{2\left(33+181 k+8 k^{2}\right)}{3(5+k)^{2}(19+23 k)} i \hat{A}_{3} \partial \hat{G}_{11}+\frac{4}{(5+k)} i \hat{B}_{3} U^{\left(\frac{5}{2}\right)} \\
& -\frac{2(7+2 k)}{(5+k)^{2}} \hat{B}_{3} \hat{B}_{-} T_{-}^{\left(\frac{3}{2}\right)}-\frac{4}{(5+k)^{2}} \hat{B}_{3} \hat{B}_{3} \hat{G}_{11}-\frac{2\left(-228+5 k+37 k^{2}\right)}{3(5+k)^{2}(19+23 k)} i \hat{B}_{3} \partial \hat{G}_{11} \\
& +\frac{2(1+5 k)}{3(5+k)^{2}} i \hat{B}_{3} \partial U^{\left(\frac{3}{2}\right)}-\frac{\left(-79-15 k+4 k^{2}\right)}{(5+k)(19+23 k)} T^{(1)} \partial \hat{G}_{11}-\frac{2(9+k)}{(5+k)^{2}} i \partial \hat{A}_{+} \hat{G}_{21} \\
& -\frac{2(37+8 k)}{3(5+k)^{2}} i \partial \hat{A}_{+} T_{+}^{\left(\frac{3}{2}\right)}-\frac{2(13+2 k)}{(5+k)^{2}} i \partial \hat{A}_{3} \hat{G}_{11}-\frac{10}{(5+k)} i \partial \hat{A}_{3} U^{\left(\frac{3}{2}\right)} \\
& -\frac{3(-3+k)}{(5+k)^{2}} i \partial \hat{B}_{-} \hat{G}_{12}-\frac{2(6+k)}{(5+k)^{2}} i \partial \hat{B}_{3} \hat{G}_{11}-\frac{1}{(5+k)} \partial T^{(1)} \hat{G}_{11} \\
& -\frac{(-9+2 k)}{3(5+k)^{2}} \partial^{2} \hat{G}_{11}-\partial U^{\left(\frac{5}{2}\right)}+\frac{2(29+k)}{3(5+k)^{2}} i \hat{A}_{3} \partial U^{\left(\frac{3}{2}\right)}+\frac{2}{(5+k)} \hat{G}_{21} U_{-}^{(2)} \\
& -\frac{2}{3(5+k)} i T_{+}^{\left(\frac{3}{2}\right)} \partial \hat{A}_{+}-\frac{8\left(-21-64 k+32 k^{2}+7 k^{3}\right)}{(3+7 k)(19+23 k)(5+k)} \hat{G}_{11} \hat{T} \\
& \left.+\frac{4}{(5+k)} \hat{G}_{11} T^{(2)}-\frac{16(-3+k)}{(19+23 k)} U^{\left(\frac{3}{2}\right)} \hat{T}\right](w)+\cdots,
\end{aligned}
$$




$$
\begin{aligned}
& \hat{G}_{12}(z) W^{(3)}(w)=\frac{1}{(z-w)^{3}}\left[\frac{2\left(-2697-242 k+1293 k^{2}+110 k^{3}\right)}{3(5+k)^{2}(19+23 k)} \hat{G}_{12}\right. \\
& \left.-\frac{2(-3+k)\left(1603+1363 k+220 k^{2}\right)}{3(5+k)^{2}(19+23 k)} T_{-}^{\left(\frac{3}{2}\right)}\right](w) \\
& +\frac{1}{(z-w)^{2}}\left[\frac{(22+5 k)}{(5+k)} W_{-}^{\left(\frac{5}{2}\right)}+\frac{2(7+k)}{(5+k)^{2}} i \hat{A}_{+} \hat{G}_{22}-\frac{8(-3+k)}{(19+23 k)} \partial T_{-}^{\left(\frac{3}{2}\right)}\right. \\
& +\frac{2(13+3 k)}{(5+k)^{2}} i \hat{A}_{+} V^{\left(\frac{3}{2}\right)}-\frac{4\left(-31+52 k+27 k^{2}\right)}{(5+k)^{2}(19+23 k)} i \hat{A}_{3} \hat{G}_{12} \\
& -\frac{2(11+3 k)}{(5+k)^{2}} i \hat{A}_{3} T_{-}^{\left(\frac{3}{2}\right)}+\frac{(17+k)}{(5+k)^{2}} i \hat{B}_{+} \hat{G}_{11}-\frac{2(13+3 k)}{(5+k)^{2}} i \hat{B}_{+} U^{\left(\frac{3}{2}\right)} \\
& -\frac{24\left(19+48 k+5 k^{2}\right)}{(5+k)^{2}(19+23 k)} i \hat{B}_{3} \hat{G}_{12}+\frac{2(11+3 k)}{(5+k)^{2}} i \hat{B}_{3} T_{-}^{\left(\frac{3}{2}\right)} \\
& \left.-\frac{12(-3+k)}{(19+23 k)} T^{(1)} \hat{G}_{12}+\frac{\left(349+2102 k+405 k^{2}+12 k^{3}\right)}{3(5+k)^{2}(19+23 k)} \partial \hat{G}_{12}\right](w) \\
& +\frac{1}{(z-w)}\left[\frac{4}{(5+k)^{2}} \hat{A}_{-} \hat{A}_{+} T_{-}^{\left(\frac{3}{2}\right)}-\frac{8}{(5+k)^{2}} \hat{B}_{+} \hat{A}_{+} \hat{G}_{21}-\frac{2(15+7 k)}{3(5+k)^{2}} \hat{A}_{-} \hat{A}_{+} \hat{G}_{12}\right. \\
& +\frac{8}{(5+k)^{2}} \hat{B}_{+} \hat{A}_{3} U^{\left(\frac{3}{2}\right)}+\frac{16}{(5+k)^{2}} \hat{B}_{+} \hat{B}_{-} \hat{G}_{12}-\frac{8(9+2 k)}{3(5+k)^{2}} \hat{B}_{+} \hat{B}_{-} T_{-}^{\left(\frac{3}{2}\right)} \\
& -\frac{(3+k)}{(5+k)^{2}} \hat{B}_{+} \hat{B}_{3} \hat{G}_{11}+\frac{4(15+4 k)}{3(5+k)^{2}} \hat{B}_{-} \hat{B}_{+} T_{-}^{\left(\frac{3}{2}\right)}-\frac{8}{(5+k)^{2}} \hat{B}_{+} \hat{B}_{3} U^{\left(\frac{3}{2}\right)} \\
& +\frac{2(15+7 k)}{3(5+k)^{2}} \hat{A}_{+} \hat{A}_{-} \hat{G}_{12}+\frac{1}{(5+k)} i \hat{B}_{+} \hat{B}_{3} \partial \hat{G}_{11}+\frac{6}{(5+k)} \hat{A}_{+} \hat{A}_{3} V^{\left(\frac{3}{2}\right)} \\
& +\frac{2}{(5+k)} i \hat{B}_{+} \partial U^{\left(\frac{3}{2}\right)}-\frac{8}{(5+k)^{2}} \hat{A}_{+} \hat{B}_{3} V^{\left(\frac{3}{2}\right)}+\frac{2(1+k)}{(5+k)^{2}} i \hat{A}_{+} \partial \hat{G}_{22} \\
& -\frac{2}{(5+k)} i \hat{A}_{+} \partial V^{\left(\frac{3}{2}\right)}-\frac{12}{(5+k)^{2}} \hat{B}_{-} \hat{B}_{+} \hat{G}_{12}+\frac{(3+k)}{(5+k)^{2}} \hat{B}_{3} \hat{B}_{+} \hat{G}_{11} \\
& -\frac{8\left(-19+2 k+5 k^{2}\right)}{(5+k)^{2}(19+23 k)} i \hat{B}_{3} \partial \hat{G}_{12}-\frac{2}{(5+k)} i \hat{B}_{3} \partial T_{-}^{\left(\frac{3}{2}\right)}+\frac{4(27+7 k)}{3(5+k)^{2}} i \hat{G}_{12} \partial \hat{A}_{3} \\
& -\frac{6}{(5+k)} i T_{-}^{\left(\frac{3}{2}\right)} \partial \hat{A}_{3}-\frac{2 k}{(5+k)^{2}} \hat{A}_{+} \hat{A}_{3} \hat{G}_{22}+\frac{2}{(5+k)} \hat{G}_{11} V_{-}^{(2)} \\
& -\frac{2}{(5+k)} \hat{G}_{22} U_{-}^{(2)}+\frac{2 k}{(5+k)^{2}} \hat{A}_{3} \hat{A}_{+} \hat{G}_{22}+\frac{(6+k)}{(5+k)} \partial W_{-}^{\left(\frac{5}{2}\right)} \\
& -\frac{2(11+3 k)}{(5+k)^{2}} \hat{A}_{3} \hat{A}_{+} V^{\left(\frac{3}{2}\right)}-\frac{16(-3+k)}{(19+23 k)} T_{-}^{\left(\frac{3}{2}\right)} \hat{T}-\frac{4\left(53+94 k+9 k^{2}\right)}{(5+k)^{2}(19+23 k)} \hat{A}_{+} \partial \hat{G}_{12} \\
& +\frac{2}{(5+k)} i \hat{A}_{+} \partial T_{-}^{\left(\frac{3}{2}\right)}-\frac{4(-3+k)}{(19+232 k)} T^{(1)} \partial \hat{G}_{12}+\frac{4\left(-27+23 k+2 k^{2}\right)}{(5+k)(19+23 k)} \hat{G}_{12} \hat{T} \\
& \left.-\frac{2(27+7 k)}{3(5+k)^{2}} i T_{-}^{\left(\frac{3}{2}\right)} \partial \hat{B}_{3}-\frac{2(11+3 k)}{(5+k)^{2}} U^{\left(\frac{3}{2}\right)} \partial \hat{B}_{+}\right](w)+\cdots, \\
& \hat{G}_{21}(z) W^{(3)}(w)=\frac{1}{(z-w)^{3}}\left[-\frac{2\left(-2697-242 k+1293 k^{2}+110 k^{3}\right)}{3(5+k)^{2}(19+23 k)} \hat{G}_{21}\right. \\
& \left.-\frac{2(-3+k)\left(1603+1363 k+220 k^{2}\right)}{3(5+k)^{2}(19+23 k)} T_{+}^{\left(\frac{3}{2}\right)}\right](w) \\
& +\frac{1}{(z-w)^{2}}\left[-\frac{(24+5 k)}{(5+k)} W_{+}^{\left(\frac{5}{2}\right)}-\frac{2(-3+k)}{(5+k)^{2}} i \hat{A}_{-} \hat{G}_{11}\right. \\
& -\frac{2(23+7 k)}{(5+k)^{2}} i \hat{A}_{-} U^{\left(\frac{3}{2}\right)}-\frac{12\left(-23+2 k+9 k^{2}\right)}{(5+k)^{2}(19+23 k)} i \hat{A}_{3} \hat{G}_{21}
\end{aligned}
$$




$$
\begin{aligned}
& +\frac{2(17+5 k)}{(5+k)^{2}} i \hat{A}_{3} T_{+}^{\left(\frac{3}{2}\right)}-\frac{(-3+k)}{(5+k)^{2}} i \hat{B}_{-} \hat{G}_{22}+\frac{2(29+5 k)}{(5+k)^{2}} i \hat{B}_{-} V^{\left(\frac{3}{2}\right)} \\
& -\frac{40\left(19+38 k+3 k^{2}\right)}{(5+k)^{2}(19+23 k)} i \hat{B}_{3} \hat{G}_{21}-\frac{2(17+5 k)}{(5+k)^{2}} i \hat{B}_{3} T_{+}^{\left(\frac{3}{2}\right)} \\
& -\frac{\left(615+2386 k+359 k^{2}+12 k^{3}\right)}{3(5+k)^{2}(19+23 k)} \partial \hat{G}_{21}-\frac{12(-3+k)}{(19+23 k)} T^{(1)} \hat{G}_{21} \\
& \left.-\frac{4(-3+k)\left(131+37 k+6 k^{2}\right)}{3(5+k)^{2}(19+23 k)} \partial T_{+}^{\left(\frac{3}{2}\right)}\right](w) \\
& +\frac{1}{(z-w)}\left[-\frac{4}{(5+k)^{2}} \hat{A}_{-} \hat{A}_{+} \hat{G}_{21}-\frac{2}{(5+k)} i \hat{A}_{-} U^{\left(\frac{5}{2}\right)}\right. \\
& +\frac{8}{(5+k)^{2}} \hat{A}_{-} \hat{B}_{-} \hat{G}_{12}-\frac{8}{(5+k)^{2}} \hat{A}_{-} \hat{B}_{3} U^{\left(\frac{3}{2}\right)}+\frac{2(-3+k)}{3(5+k)^{2}} i \hat{A}_{-} \partial \hat{G}_{11} \\
& -\frac{2(-7+k)}{3(5+k)^{2}} \hat{A}_{-} \partial U^{\left(\frac{3}{2}\right)}+\frac{4}{(5+k)^{2}} \hat{B}_{+} \hat{B}_{-} \hat{G}_{21}+\frac{2}{(5+k)} i \hat{B}_{+} V^{\left(\frac{5}{2}\right)} \\
& +\frac{8}{(5+k)^{2}} \hat{B}_{+} \hat{A}_{3} V^{\left(\frac{3}{2}\right)}-\frac{8}{(5+k)^{2}} \hat{B}_{-} \hat{B}_{+} \hat{G}_{21}-\frac{6}{(5+k)} \hat{B}_{-} \hat{B}_{3} V^{\left(\frac{3}{2}\right)} \\
& +\frac{(-3+k)}{3(5+k)^{2}} i \hat{B}_{-} \partial \hat{G}_{22}-\frac{2(1+k)}{3(5+k)^{2}} i \hat{B}_{-} \partial V^{\left(\frac{3}{2}\right)}+\frac{8}{(5+k)^{2}} \hat{A}_{-} \hat{A}_{3} U^{\left(\frac{3}{2}\right)} \\
& -\frac{4\left(-23+2 k+9 k^{2}\right)}{(5+k)^{2}(19+23 k)} i \hat{A}_{3} \partial \hat{G}_{21}+\frac{2}{(5+k)} i \hat{A}_{3} \partial T_{+}^{\left(\frac{3}{2}\right)}+\frac{2(11+3 k)}{(5+k)^{2}} \hat{B}_{3} \hat{B}_{-} V^{\left(\frac{3}{2}\right)} \\
& -\frac{8\left(19+48 k+5 k^{2}\right)}{(5+k)^{2}(19+23 k)} i \hat{B}_{3} \partial \hat{G}_{21}-\frac{2}{(5+k)} i \hat{B}_{3} \partial T_{+}^{\left(\frac{3}{2}\right)}-\frac{4(-3+k)}{(19+23 k)} T^{(1)} \partial \hat{G}_{21} \\
& -\frac{(4+k)}{(5+k)} \partial W_{+}^{\left(\frac{5}{2}\right)}-\frac{2(1+k)}{(5+k)^{2}} i \partial \hat{A}_{-} \hat{G}_{11}+\frac{8}{(5+k)^{2}} i \partial \hat{A}_{3} \hat{G}_{21} \\
& -\frac{2}{(5+k)} i \partial \hat{B}_{3} T_{+}^{\left(\frac{3}{2}\right)}-\frac{(29+5 k)}{6(5+k)^{2}} \partial^{2} \hat{G}_{21}-\frac{4(9+2 k)}{3(5+k)^{2}} \partial^{2} T_{+}^{\left(\frac{3}{2}\right)} \\
& -\frac{4\left(-27+23 k+2 k^{2}\right)}{(5+k)(19+23 k)} \hat{G}_{21} \hat{T}-\frac{16(-3+k)}{(19+23 k)} T_{+}^{\left(\frac{3}{2}\right)} \hat{T}-\frac{2(11+3 k)}{(5+k)^{2}} i U^{\left(\frac{3}{2}\right)} \partial \hat{A}_{-} \\
& \left.-\frac{1}{(5+k)} i \hat{G}_{22} \partial \hat{B}_{-}\right](w)+\cdots \\
& \hat{G}_{22}(z) W^{(3)}(w)=\frac{1}{(z-w)^{3}}\left[\frac{2(-3+k)\left(571+357 k+110 k^{2}\right)}{3(5+k)^{2}(19+23 k)} \hat{G}_{22}\right. \\
& \left.-\frac{2(-3+k)\left(1489+1225 k+220 k^{2}\right)}{3(5+k)^{2}(19+23 k)} V^{\left(\frac{3}{2}\right)}\right](w) \\
& +\frac{1}{(z-w)^{2}}\left[\frac{(19+5 k)}{(5+k)} V^{\left(\frac{5}{2}\right)}-\frac{2(14+k)}{(5+k)^{2}} i \hat{A}_{-} \hat{G}_{12}+\frac{2(3+2 k)}{(5+k)^{2}} i \hat{A}_{-} T_{-}^{\left(\frac{3}{2}\right)}\right. \\
& -\frac{2\left(109+349 k+100 k^{2}\right)}{(5+k)^{2}(19+23 k)} i \hat{A}_{3} \hat{G}_{22}+\frac{2(-11+k)}{(5+k)^{2}} i \hat{A}_{3} V^{\left(\frac{3}{2}\right)} \\
& +\frac{(29+k)}{(5+k)^{2}} i \hat{B}_{+} \hat{G}_{21}+\frac{2(14+3 k)}{(5+k)^{2}} i \hat{B}_{+} T_{+}^{\left(\frac{3}{2}\right)}-\frac{2\left(76+373 k+37 k^{2}\right)}{(5+k)^{2}(19+23 k)} i \hat{B}_{3} \hat{G}_{22} \\
& +\frac{2(33+5 k)}{(5+k)^{2}} i \hat{B}_{3} V^{\left(\frac{3}{2}\right)}-\frac{\left(-161+47 k+12 k^{2}\right)}{(5+k)(19+23 k)} T^{(1)} \hat{G}_{22} \\
& +\frac{\left(-1389-512 k+113 k^{2}+12 k^{3}\right)}{3(5+k)^{2}(19+23 k)} \partial \hat{G}_{22} \\
& \left.-\frac{4\left(-89+445 k+88 k^{2}+6 k^{3}\right)}{3(5+k)^{2}(19+23 k)} \partial V^{\left(\frac{3}{2}\right)}\right](w)
\end{aligned}
$$




$$
\begin{aligned}
& +\frac{1}{(z-w)}\left[\frac{8}{(5+k)^{2}} \hat{A}_{-} \hat{A}_{+} \hat{G}_{22}-\frac{2}{(5+k)} i \hat{A}_{-} W_{-}^{\left(\frac{5}{2}\right)}+\frac{2(10+k)}{(5+k)^{2}} \hat{A}_{-} \hat{A}_{3} \hat{G}_{12}\right. \\
& -\frac{4}{(5+k)^{2}} \hat{A}_{-} \hat{B}_{+} U^{\left(\frac{3}{2}\right)}+\frac{8}{(5+k)^{2}} \hat{A}_{-} \hat{B}_{3} T_{-}^{\left(\frac{3}{2}\right)}+\frac{2(2+k)}{3(5+k)^{2}} i \hat{A}_{-} \partial \hat{G}_{12} \\
& -\frac{2(15+2 k)}{3(5+k)^{2}} \hat{A}_{-} \partial T_{-}^{\left(\frac{3}{2}\right)}+\frac{(10+3 k)}{(5+k)^{2}} \hat{B}_{+} \hat{B}_{-} \hat{G}_{22}-\frac{5}{(5+k)} \hat{B}_{+} \hat{B}_{-} V^{\left(\frac{3}{2}\right)} \\
& -\frac{8}{(5+k)^{2}} \hat{B}_{+} \hat{B}_{3} \hat{G}_{21}+\frac{(1+k)}{(5+k)^{2}} i \hat{B}_{+} \partial \hat{G}_{21}-\frac{2(6+k)}{(5+k)^{2}} i \hat{B}_{+} \partial T_{+}^{\left(\frac{3}{2}\right)} \\
& -\frac{3(2+k)}{(5+k)^{2}} \hat{B}_{-} \hat{B}_{+} \hat{G}_{22}+\frac{(21+5 k)}{(5+k)^{2}} \hat{B}_{-} \hat{B}_{+} V^{\left(\frac{3}{2}\right)}-\frac{4}{(5+k)} i \hat{A}_{3} V^{\left(\frac{5}{2}\right)} \\
& -\frac{2(10+k)}{(5+k)^{2}} \hat{A}_{3} \hat{A}_{-} \hat{G}_{12}-\frac{16(-3+k)}{(19+23 k)} V^{\left(\frac{3}{2}\right)} \hat{T} \\
& +\frac{4}{(5+k)^{2}} \hat{A}_{3} \hat{A}_{3} \hat{G}_{22}-\frac{8}{(5+k)^{2}} \hat{A}_{3} \hat{B}_{3} \hat{G}_{22}+\frac{16}{(5+k)^{2}} \hat{A}_{3} \hat{B}_{3} V^{\left(\frac{3}{2}\right)} \\
& -\frac{2\left(375+595 k+8 k^{2}\right)}{3(5+k)^{2}(19+23 k)} i \hat{A}_{3} \partial \hat{G}_{22}-\frac{2(13+k)}{3(5+k)^{2}} i \hat{A}_{3} \partial V^{\left(\frac{3}{2}\right)} \\
& +\frac{4}{(5+k)} i \hat{B}_{3} V^{\left(\frac{5}{2}\right)}+\frac{4}{(5+k)^{2}} \hat{B}_{3} \hat{B}_{3} \hat{G}_{22}-\frac{16}{(5+k)^{2}} \hat{B}_{3} \hat{B}_{3} V^{\left(\frac{3}{2}\right)} \\
& +\frac{2\left(228+109 k+101 k^{2}\right)}{3(5+k)^{2}(19+23 k)} i \hat{B}_{3} \partial \hat{G}_{22}-\frac{2(17+5 k)}{3(5+k)^{2}} i \hat{B}_{3} \partial V^{\left(\frac{3}{2}\right)} \\
& -\frac{\left(-41+31 k+4 k^{2}\right)}{(5+k)(19+23 k)} T^{(1)} \partial \hat{G}_{22}+\partial V^{\left(\frac{5}{2}\right)}+\frac{2(7+2 k)}{(5+k)^{2}} i \partial \hat{A}_{-} T_{-}^{\left(\frac{3}{2}\right)} \\
& -\frac{2(7+2 k)}{(5+k)^{2}} i \partial \hat{A}_{3} \hat{G}_{22}-\frac{2}{(5+k)} i \partial \hat{A}_{3} V^{\left(\frac{3}{2}\right)}-\frac{(-29+7 k)}{3(5+k)^{2}} i \partial \hat{B}_{+} \hat{G}_{21} \\
& +\frac{2(37+8 k)}{3(5+k)^{2}} i \partial \hat{B}_{+} T_{+}^{\left(\frac{3}{2}\right)}+\frac{1}{(5+k)} \partial T^{(1)} \hat{G}_{22}-\frac{2}{(5+k)} \hat{G}_{21} V_{-}^{(2)} \\
& +\frac{2(23+2 k)}{3(5+k)^{2}} \hat{G}_{21} \partial \hat{B}_{+}+\frac{2}{3(5+k)} i T_{+}^{\left(\frac{3}{2}\right)} \partial \hat{B}_{+} \\
& \left.+\frac{8}{(3+7 k)(19+23 k)(5+k)} \hat{G}_{22} \hat{T}-\frac{4}{(5+k)} \hat{G}_{22} T^{(2)}\right](w)+\cdots .
\end{aligned}
$$

The U(1) charge conservation in these OPEs can be checked from tables 2, 4, 6 and 7.

\section{C.4 The OPEs between four spin $\frac{3}{2}$ currents and the higher spin currents in different basis}

Let us present the OPEs in different basis as we did in appendix (B.4). The OPEs between the spin- $\frac{3}{2}$ currents $\hat{G}_{a}(z)$ and the spin- 1 current $\hat{T}^{(1)}(w)=T^{(1)}(w)$ given by (C.1) and (C.2) can be rewritten in terms of hatted higher spins in (6.8) as follows:

$$
\begin{aligned}
& \left(\begin{array}{c}
\hat{G}_{11} \\
\hat{G}_{22}
\end{array}\right)(z) \hat{T}^{(1)}(w)=\frac{1}{(z-w)} 2\left(\begin{array}{c}
\hat{U}^{\left(\frac{3}{2}\right)} \\
\hat{V}^{\left(\frac{3}{2}\right)}
\end{array}\right)(w)+\cdots, \\
& \left(\begin{array}{c}
\hat{G}_{12} \\
\hat{G}_{21}
\end{array}\right)(z) \hat{T}^{(1)}(w)=\frac{1}{(z-w)} 2\left(\begin{array}{c}
\hat{T}_{-}^{\left(\frac{3}{2}\right)} \\
\hat{T}_{+}^{\left(\frac{3}{2}\right)}
\end{array}\right)(w)+\cdots .
\end{aligned}
$$

There are no $\hat{G}_{a}(w)$ dependences in the right hand side of (C.47). The spin-1 current $\hat{T}^{(1)}(z)$ acting on the four spin- $\frac{3}{2}$ currents of large $\mathcal{N}=4$ nonlinear algebra leads to the four currents with same spins but they are located at higher spin multiplet. 
The OPEs between the spin- $\frac{3}{2}$ currents $\hat{G}_{a}(z)$ and the four spin- $\frac{3}{2}$ currents in the right hand side of (C.47) or (6.8) can be described by

$$
\begin{aligned}
& \left(\begin{array}{l}
\hat{G}_{11} \\
\hat{G}_{22}
\end{array}\right)(z) \hat{T}_{ \pm}^{\left(\frac{3}{2}\right)}(w)=-\frac{1}{(z-w)}\left(\begin{array}{l}
\hat{U}_{+}^{(2)}+\frac{i}{3} \hat{T}^{(1)} \hat{B}_{-} \\
V_{-}^{(2)}-\frac{i}{3} \hat{T}^{(1)} \hat{B}_{+}
\end{array}\right)(w)+\cdots, \\
& \left(\begin{array}{l}
\hat{G}_{12} \\
\hat{G}_{21}
\end{array}\right)(z) \hat{T}_{ \pm}^{\left(\frac{3}{2}\right)}(w)=\frac{1}{(z-w)^{2}} \hat{T}^{(1)}(w)+\frac{1}{(z-w)}\left(\begin{array}{c}
\left\{\hat{G}_{12} \hat{T}_{+}^{\left(\frac{3}{2}\right)}\right\}_{-1} \\
\left\{\hat{G}_{21} \hat{T}_{-}^{\left(\frac{3}{2}\right)}\right\}_{-1}
\end{array}\right)(w)+\cdots, \\
& \left(\begin{array}{c}
\hat{G}_{22} \\
\hat{G}_{11}
\end{array}\right)(z) \hat{T}_{ \pm}^{\left(\frac{3}{2}\right)}(w)=-\frac{1}{(z-w)}\left(\begin{array}{c}
\hat{V}_{+}^{(2)}+\frac{i}{k} \hat{T}^{(1)} \hat{A}_{-} \\
\hat{U}_{-}^{(2)}-\frac{i}{k} \hat{T}^{(1)} \hat{A}_{+}
\end{array}\right)(w)+\cdots, \\
& \left(\begin{array}{l}
\hat{G}_{12} \\
\hat{G}_{21}
\end{array}\right)(z)\left(\begin{array}{c}
\hat{U}^{\left(\frac{3}{2}\right)} \\
\hat{V}^{\left(\frac{3}{2}\right)}
\end{array}\right)(w)=\frac{1}{(z-w)}\left(\begin{array}{c}
\hat{U}_{-}^{(2)}-\frac{i}{k} \hat{T}^{(1)} \hat{A}_{+} \\
\hat{V}_{+}^{(2)}+\frac{i}{k} \hat{T}^{(1)} \hat{A}_{-}
\end{array}\right)(w)+\cdots, \\
& \left(\begin{array}{l}
\hat{G}_{21} \\
\hat{G}_{12}
\end{array}\right)(z)\left(\begin{array}{c}
\hat{U}^{\left(\frac{3}{2}\right)} \\
\hat{V}^{\left(\frac{3}{2}\right)}
\end{array}\right)(w)=\frac{1}{(z-w)}\left(\begin{array}{c}
\hat{U}_{+}^{(2)}+\frac{i}{3} \hat{T}^{(1)} \hat{B}_{-} \\
\hat{V}_{-}^{(2)}-\frac{i}{3} \hat{T}^{(1)} \hat{B}_{+}
\end{array}\right)(w)+\cdots, \\
& \left(\begin{array}{c}
\hat{G}_{22} \\
\hat{G}_{11}
\end{array}\right)(z)\left(\begin{array}{c}
\hat{U}^{\left(\frac{3}{2}\right)} \\
\hat{V}^{\left(\frac{3}{2}\right)}
\end{array}\right)(w)=\frac{1}{(z-w)^{2}} \hat{T}^{(1)}(w)+\frac{1}{(z-w)}\left(\begin{array}{l}
\left\{\hat{G}_{22} \hat{U}^{\left(\frac{3}{2}\right)}\right\}_{-1} \\
\left\{\hat{G}_{11} \hat{V}^{\left(\frac{3}{2}\right)}\right\}_{-1}
\end{array}\right)(w) \\
& +\cdots \text {. }
\end{aligned}
$$

All the third-order poles appeared in previous basis are disappeared in this new basis (C.48). From appendix (C.48), one sees the six higher spin-2 currents appear in the right hand side. Note that the fields $\hat{T}^{(1)} \hat{A}_{ \pm}(w)$ and $\hat{T}^{(1)} \hat{B}_{ \pm}(w)$ are primary fields under the stress energy tensor $\hat{T}(z)$ because $\hat{T}^{(1)}(z)$ commutes with both $\hat{A}_{ \pm}(w)$ and $\hat{B}_{ \pm}(w)$ and these five spin-1 currents are primary. The spin-1 current $\hat{T}^{(1)}(w)$ appears in the second-order poles of second and sixth OPEs in appendix (C.48).

The first-order poles in appendix (C.48) are given by

$$
\begin{aligned}
\left(\begin{array}{c}
\left\{\hat{G}_{12} \hat{T}_{+}^{\left(\frac{3}{2}\right)}\right\}_{-1} \\
\left\{\hat{G}_{21} \hat{T}_{-}^{\left(\frac{3}{2}\right)}\right\}_{-1}
\end{array}\right)(w) & =\mp \frac{1}{2}\left(\hat{T}^{(2)}+\hat{W}^{(2)}\right)(w) \mp \frac{1}{2}\left(\hat{T}^{(2)}-\hat{W}^{(2)}\right)(w) \\
& \pm \frac{i}{k} \hat{T}^{(1)} \hat{A}_{3}(w) \pm \frac{i}{3} \hat{T}^{(1)} \hat{B}_{3}(w)+\frac{1}{2} \partial \hat{T}^{(1)}(w) \pm \frac{(k+3)}{(7 k+3)} \tilde{\hat{T}}(w) \\
\left(\begin{array}{l}
\left\{\hat{G}_{22} \hat{U}^{\left(\frac{3}{2}\right)}\right\}_{-1} \\
\left\{\hat{G}_{11} \hat{V}^{\left(\frac{3}{2}\right)}\right\}_{-1}
\end{array}\right)(w) & =\mp \frac{1}{2}\left(\hat{T}^{(2)}+\hat{W}^{(2)}\right)(w) \pm \frac{1}{2}\left(\hat{T}^{(2)}-\hat{W}^{(2)}\right)(w) \\
& \mp \frac{i}{k} \hat{T}^{(1)} \hat{A}_{3}(w) \pm \frac{i}{3} \hat{T}^{(1)} \hat{B}_{3}(w)+\frac{1}{2} \partial \hat{T}^{(1)}(w) \pm \tilde{\hat{T}}(w) .
\end{aligned}
$$

In (C.49), each five term is primary field except the descendant field $\frac{1}{2} \partial \hat{T}^{(1)}(w)$ which should be present due to the second-order pole term, $\hat{T}^{(1)}(w)$, and moreover $\tilde{\hat{T}}(w)$ was defined in (B.35) previously. 
Now one calculates the OPEs between the spin- $\frac{3}{2}$ currents $\hat{G}_{a}(z)$ and the above six spin-2 currents in the right hand side of appendix (C.48) or (6.8)

$$
\begin{aligned}
& \hat{G}_{11}(z)\left(\begin{array}{c}
\hat{U}_{-}^{(2)} \\
\hat{T}^{(2)}-\hat{W}^{(2)} \\
\hat{V}_{+}^{(2)}
\end{array}\right)(w)=\frac{1}{(z-w)^{2}}\left[\frac{2(-1+k)(5+2 k)}{k(5+k)}\right]\left(\begin{array}{c}
0 \\
\hat{U}^{\left(\frac{3}{2}\right)} \\
-\hat{T}_{+}^{\left(\frac{3}{2}\right)}
\end{array}\right)(w) \\
& +\frac{1}{(z-w)}\left(\begin{array}{c}
\frac{2 i(2 k+5)}{k(k+5)} \hat{A}_{+} \hat{U}^{\left(\frac{3}{2}\right)} \\
\left\{\hat{G}_{11}\left(\hat{T}^{(2)}-\hat{W}^{(2)}\right)\right\}_{-1} \\
\left\{\hat{G}_{11} \hat{V}_{+}^{(2)}\right\}_{-1}
\end{array}\right)(w)+\cdots \\
& \hat{G}_{12}(z)\left(\begin{array}{c}
\hat{U}_{-}^{(2)} \\
\hat{T}^{(2)}-\hat{W}^{(2)} \\
\hat{V}_{+}^{(2)}
\end{array}\right)(w)=\frac{1}{(z-w)^{2}}\left[\frac{2(-1+k)(5+2 k)}{k(5+k)}\right]\left(\begin{array}{c}
0 \\
\hat{T}_{-}^{\left(\frac{3}{2}\right)} \\
\hat{V}^{\left(\frac{3}{2}\right)}
\end{array}\right)(w) \\
& +\frac{1}{(z-w)}\left(\begin{array}{c}
\frac{2 i(2 k+5)}{k(k+5)} \hat{A}_{+} \hat{T}_{-}^{\left(\frac{3}{2}\right)} \\
\left\{\hat{G}_{12}\left(\hat{T}^{(2)}-\hat{W}^{(2)}\right)\right\}_{-1} \\
\left\{\hat{G}_{12} \hat{V}_{+}^{(2)}\right\}_{-1}
\end{array}\right)(w)+\cdots \\
& \hat{G}_{21}(z)\left(\begin{array}{c}
\hat{U}_{-}^{(2)} \\
\hat{T}^{(2)}-\hat{W}^{(2)} \\
\hat{V}_{+}^{(2)}
\end{array}\right)(w)=\frac{1}{(z-w)^{2}}\left[\frac{2(-1+k)(5+2 k)}{k(5+k)}\right]\left(\begin{array}{c}
\hat{U}^{\left(\frac{3}{2}\right)} \\
-\hat{T}_{+}^{\left(\frac{3}{2}\right)} \\
0
\end{array}\right)(w) \\
& +\frac{1}{(z-w)}\left(\begin{array}{c}
\left\{\hat{G}_{21} \hat{U}_{-}^{(2)}\right\}_{-1} \\
\left\{\hat{G}_{21}\left(\hat{T}^{(2)}-\hat{W}^{(2)}\right)\right\}_{-1} \\
-\frac{2 i(5+2 k)}{k(5+k)} \hat{A}_{-} \hat{T}_{+}^{\left(\frac{3}{2}\right)}
\end{array}\right)(w)+\cdots \\
& \hat{G}_{22}(z)\left(\begin{array}{c}
\hat{U}_{-}^{(2)} \\
\hat{T}^{(2)}-\hat{W}^{(2)} \\
\hat{V}_{+}^{(2)}
\end{array}\right)(w)=\frac{1}{(z-w)^{2}}\left[\frac{2(-1+k)(5+2 k)}{k(5+k)}\right]\left(\begin{array}{c}
-\hat{T}_{-}^{\left(\frac{3}{2}\right)} \\
-\hat{V}^{\left(\frac{3}{2}\right)} \\
0
\end{array}\right)(w) \\
& +\frac{1}{(z-w)}\left(\begin{array}{c}
\left\{\hat{G}_{22} \hat{U}_{-}^{(2)}\right\}_{-1} \\
\left\{\hat{G}_{22}\left(\hat{T}^{(2)}-\hat{W}^{(2)}\right)\right\}_{-1} \\
-\frac{2 i(5+2 k)}{k(5+k)} \hat{A}_{-} \hat{V}^{\left(\frac{3}{2}\right)}
\end{array}\right)(w)+\cdots \\
& \hat{G}_{11}(z)\left(\begin{array}{c}
\hat{V}_{-}^{(2)} \\
\hat{T}^{(2)}+\hat{W}^{(2)} \\
\hat{U}_{+}^{(2)}
\end{array}\right)(w)=\frac{1}{(z-w)^{2}}\left[\frac{4(8+k)}{3(5+k)}\right]\left(\begin{array}{c}
-\hat{T}_{-}^{\left(\frac{3}{2}\right)} \\
-\hat{U}^{\left(\frac{3}{2}\right)} \\
0
\end{array}\right)(w) \\
& +\frac{1}{(z-w)}\left(\begin{array}{c}
\left\{\hat{G}_{11} \hat{V}_{-}^{(2)}\right\}_{-1} \\
\left\{\hat{G}_{11}\left(\hat{T}^{(2)}+\hat{W}^{(2)}\right)\right\}_{-1} \\
-\frac{2 i(8+k)}{3(5+k)} \hat{B}_{-} \hat{U}^{\left(\frac{3}{2}\right)}
\end{array}\right)(w)+\cdots,
\end{aligned}
$$




$$
\begin{aligned}
& \hat{G}_{12}(z)\left(\begin{array}{c}
\hat{V}_{-}^{(2)} \\
\hat{T}^{(2)}+\hat{W}^{(2)} \\
\hat{U}_{+}^{(2)}
\end{array}\right)(w)=\frac{1}{(z-w)^{2}}\left[\frac{4(8+k)}{3(5+k)}\right]\left(\begin{array}{c}
0 \\
\hat{T}_{-}^{\left(\frac{3}{2}\right)} \\
\hat{U}^{\left(\frac{3}{2}\right)}
\end{array}\right)(w) \\
& +\frac{1}{(z-w)}\left(\begin{array}{c}
\frac{2 i(8+k)}{3(5+k)} \hat{B}_{+} T_{-}^{\left(\frac{3}{2}\right)} \\
\left\{\hat{G}_{12}\left(\hat{T}^{(2)}+\hat{W}^{(2)}\right)\right\}_{-1} \\
\left\{\hat{G}_{12} \hat{U}_{+}^{(2)}\right\}_{-1}
\end{array}\right)(w)+\cdots \\
& \hat{G}_{21}(z)\left(\begin{array}{c}
\hat{V}_{-}^{(2)} \\
\hat{T}^{(2)}+\hat{W}^{(2)} \\
\hat{U}_{+}^{(2)}
\end{array}\right)(w)=\frac{1}{(z-w)^{2}}\left[\frac{4(8+k)}{3(5+k)}\right]\left(\begin{array}{c}
\hat{V}^{\left(\frac{3}{2}\right)} \\
-\hat{T}_{+}^{\left(\frac{3}{2}\right)} \\
0
\end{array}\right)(w) \\
& +\frac{1}{(z-w)}\left(\begin{array}{c}
\left\{\hat{G}_{21} \hat{V}_{-}^{(2)}\right\}_{-1} \\
\left\{\hat{G}_{21}\left(\hat{T}^{(2)}+\hat{W}^{(2)}\right)\right\}_{-1} \\
-\frac{2 i(8+k)}{3(5+k)} \hat{B}_{-} \hat{T}_{+}^{\left(\frac{3}{2}\right)}
\end{array}\right)(w)+\cdots \\
& \hat{G}_{22}(z)\left(\begin{array}{c}
\hat{V}_{-}^{(2)} \\
\hat{T}^{(2)}+\hat{W}^{(2)} \\
\hat{U}_{+}^{(2)}
\end{array}\right)(w)=\frac{1}{(z-w)^{2}}\left[\frac{4(8+k)}{3(5+k)}\right]\left(\begin{array}{c}
0 \\
\hat{V}^{\left(\frac{3}{2}\right)} \\
-\hat{T}_{+}^{\left(\frac{3}{2}\right)}
\end{array}\right)(w) \\
& +\frac{1}{(z-w)}\left(\begin{array}{c}
\frac{2 i(8+k)}{3(5+k)} \hat{B}_{+} \hat{V}^{\left(\frac{3}{2}\right)} \\
\left\{\hat{G}_{22}\left(\hat{T}^{(2)}+\hat{W}^{(2)}\right)\right\}_{-1} \\
\left\{\hat{G}_{22} \hat{U}_{+}^{(2)}\right\}_{-1}
\end{array}\right)(w)+\cdots .
\end{aligned}
$$

The $k$-dependent structure constants appearing in the second-order pole (in the right hand side) of appendix (C.50) for the $(\mathbf{3}, \mathbf{1})$ are common up to the signs and those for the $(\mathbf{1}, \mathbf{3})$ have same value up to the signs. The nonlinear quadratic terms $\left(\hat{A}_{+} \hat{U}^{\left(\frac{3}{2}\right)}(w), \cdots\right)$ appearing in the first-order poles in appendix (C.50) are primary fields because the OPEs between the corresponding spin- 1 currents and the corresponding spin- $\frac{3}{2}$ currents do not have any singular terms. For example, $\hat{A}_{+}(z) \hat{U}^{\left(\frac{3}{2}\right)}(w)=+\cdots$.

The nonlinear spin- $\frac{5}{2}$ fields appearing in (C.50) are given by as follows:

$$
\begin{aligned}
\left(\begin{array}{c}
\left\{\hat{G}_{21} \hat{U}_{-}^{(2)}\right\}_{-1} \\
\left\{\hat{G}_{12} \hat{V}_{+}^{(2)}\right\}_{-1}
\end{array}\right)(w)= & \pm\left(\begin{array}{c}
\hat{U}^{\left(\frac{5}{2}\right)} \\
\hat{V}^{\left(\frac{5}{2}\right)}
\end{array}\right)(w)+\frac{2(-1+k)(5+2 k)}{3 k(5+k)} \partial\left(\begin{array}{c}
\hat{U}^{\left(\frac{3}{2}\right)} \\
\hat{V}^{\left(\frac{3}{2}\right)}
\end{array}\right)(w) \\
& +\frac{(2 k+5)}{k(k+5)\left(38 k^{2}+41 k-25\right)}\left[\mp 2 i\left(8 k^{2}-51 k+25\right) \hat{A}_{ \pm} \hat{T}_{ \pm}^{\left(\frac{3}{2}\right)}\right. \\
& \mp 4 i k(23 k-5) \hat{A}_{3}\left(\begin{array}{c}
\hat{U}^{\left(\frac{3}{2}\right)} \\
\hat{V}^{\left(\frac{3}{2}\right)}
\end{array}\right) \mp 12 i k(k-1)(k+1) \hat{B}_{\mp} \hat{T}_{\mp}^{\left(\frac{3}{2}\right)} \\
& \mp 12 i k(k-1)(k+1) \hat{B}_{3}\left(\begin{array}{c}
\hat{U}^{\left(\frac{3}{2}\right)} \\
\hat{V}^{\left(\frac{3}{2}\right)}
\end{array}\right)
\end{aligned}
$$




$$
\begin{aligned}
& -5(k-1)(k+1)(k+5) \hat{T}^{(1)}\left(\begin{array}{c}
\hat{G}_{11} \\
\hat{G}_{22}
\end{array}\right) \\
& \left.-\frac{4}{3}(k-1)\left(7 k^{2}+4 k+25\right) \partial\left(\begin{array}{c}
\hat{U}^{\left(\frac{3}{2}\right)} \\
\hat{V}^{\left(\frac{3}{2}\right)}
\end{array}\right)\right](w), \\
& \left(\begin{array}{l}
\left\{\hat{G}_{22} \hat{U}_{-}^{(2)}\right\}_{-1} \\
\left\{\hat{G}_{11} \hat{V}_{+}^{(2)}\right\}_{-1}
\end{array}\right)(w)= \pm \hat{W}_{\mp}^{\left(\frac{5}{2}\right)}(w)-\frac{2(-1+k)(5+2 k)}{3 k(5+k)} \partial \hat{T}_{\mp}^{\left(\frac{3}{2}\right)}(w) \\
& +\frac{(2 k+5)}{k(k+5)\left(38 k^{2}+41 k-25\right)}[ \\
& \mp 2 i\left(8 k^{2}-51 k+25\right) \hat{A}_{ \pm}\left(\begin{array}{c}
\hat{V}^{\left(\frac{3}{2}\right)} \\
\hat{U}^{\left(\frac{3}{2}\right)}
\end{array}\right) \\
& \pm 4 i k(23 k-5) \hat{A}_{3} \hat{T}_{\mp}^{\left(\frac{3}{2}\right)} \pm 12 i k(k-1)(k+1) \hat{B}_{ \pm}\left(\begin{array}{c}
\hat{U}^{\left(\frac{3}{2}\right)} \\
\hat{V}^{\left(\frac{3}{2}\right)}
\end{array}\right) \\
& \mp 12 i k(k-1)(k+1) \hat{B}_{3} \hat{T}_{\mp}^{\left(\frac{3}{2}\right)} \\
& +5(k-1)(k+1)(k+5) \hat{T}^{(1)}\left(\begin{array}{c}
\hat{G}_{12} \\
\hat{G}_{21}
\end{array}\right) \\
& \left.+\frac{4}{3}(k-1)\left(7 k^{2}+4 k+25\right) \partial \hat{T}_{\mp}^{\left(\frac{3}{2}\right)}\right](w), \\
& \left(\begin{array}{l}
\left\{\hat{G}_{11}\left(\hat{T}^{(2)}-\hat{W}^{(2)}\right)\right\}_{-1} \\
\left\{\hat{G}_{22}\left(\hat{T}^{(2)}-\hat{W}^{(2)}\right)\right\}_{-1}
\end{array}\right)(w)=\left(\begin{array}{c}
\hat{U}^{\left(\frac{5}{2}\right)} \\
\hat{V}^{\left(\frac{5}{2}\right)}
\end{array}\right)(w) \pm \frac{2(-1+k)(5+2 k)}{3 k(5+k)} \partial\left(\begin{array}{c}
\hat{U}^{\left(\frac{3}{2}\right)} \\
\hat{V}^{\left(\frac{3}{2}\right)}
\end{array}\right)(w) \\
& +\frac{(2 k+5)}{k(k+5)\left(38 k^{2}+41 k-25\right)}\left[-4 i k(23 k-5) \hat{A}_{ \pm} \hat{T}_{ \pm}^{\left(\frac{3}{2}\right)}\right. \\
& +4 i\left(15 k^{2}+46 k-25\right) \hat{A}_{3}\left(\begin{array}{c}
\hat{U}^{\left(\frac{3}{2}\right)} \\
\hat{V}^{\left(\frac{3}{2}\right)}
\end{array}\right) \\
& -12 i k(k-1)(k+1) \hat{B}_{\mp} \hat{T}_{\mp}^{\left(\frac{3}{2}\right)} \\
& -12 i k(k-1)(k+1) \hat{B}_{3}\left(\begin{array}{c}
\hat{U}^{\left(\frac{3}{2}\right)} \\
\hat{V}^{\left(\frac{3}{2}\right)}
\end{array}\right) \\
& \mp 5(k-1)(k+1)(k+5) \hat{T}^{(1)}\left(\begin{array}{c}
\hat{G}_{11} \\
\hat{G}_{22}
\end{array}\right) \\
& \left.\mp \frac{4}{3}(k-1)\left(7 k^{2}+4 k+25\right) \partial\left(\begin{array}{c}
\hat{U}^{\left(\frac{3}{2}\right)} \\
\hat{V}^{\left(\frac{3}{2}\right)}
\end{array}\right)\right](w) \\
& +\frac{6 k}{(7 k+3)}\left[\mp \frac{i}{(k+2)} \hat{A}_{ \pm}\left(\begin{array}{c}
\hat{G}_{21} \\
\hat{G}_{12}
\end{array}\right) \mp \frac{i}{(k+2)} \hat{A}_{3}\left(\begin{array}{c}
\hat{G}_{11} \\
\hat{G}_{22}
\end{array}\right)\right. \\
& \pm \frac{i}{5} \hat{B}_{\mp}\left(\begin{array}{c}
\hat{G}_{12} \\
\hat{G}_{21}
\end{array}\right) \pm \frac{i}{5} \hat{B}_{3}\left(\begin{array}{c}
\hat{G}_{11} \\
\hat{G}_{22}
\end{array}\right) \\
& \left.+\partial\left(\begin{array}{l}
\hat{G}_{11} \\
\hat{G}_{22}
\end{array}\right)+\frac{3(9 k+13)}{5(k+2)} \hat{T}^{(1)}\left(\begin{array}{c}
\hat{U}^{\left(\frac{3}{2}\right)} \\
\hat{V}^{\left(\frac{3}{2}\right)}
\end{array}\right)\right](w),
\end{aligned}
$$




$$
\begin{aligned}
& \left(\begin{array}{l}
\left\{\hat{G}_{12}\left(\hat{T}^{(2)}-\hat{W}^{(2)}\right)\right\}_{-1} \\
\left\{\hat{G}_{21}\left(\hat{T}^{(2)}-\hat{W}^{(2)}\right)\right\}_{-1}
\end{array}\right)(w)=-\hat{W}_{\mp}^{\left(\frac{5}{2}\right)}(w) \pm \frac{2(-1+k)(5+2 k)}{3 k(5+k)} \partial \hat{T}_{\mp}^{\left(\frac{3}{2}\right)}(w) \\
& +\frac{(2 k+5)}{k(k+5)\left(38 k^{2}+41 k-25\right)}\left[4 i k(23 k-5) \hat{A}_{ \pm}\left(\begin{array}{c}
\hat{V}^{\left(\frac{3}{2}\right)} \\
\hat{U}^{\left(\frac{3}{2}\right)}
\end{array}\right)\right. \\
& +4 i\left(15 k^{2}+46 k-25\right) \hat{A}_{3} \hat{T}_{\mp}^{\left(\frac{3}{2}\right)} \\
& -12 i k(k-1)(k+1) \hat{B}_{ \pm}\left(\begin{array}{c}
\hat{U}^{\left(\frac{3}{2}\right)} \\
\hat{V}^{\left(\frac{3}{2}\right)}
\end{array}\right) \\
& +12 i k(k-1)(k+1) \hat{B}_{3} \hat{T}_{\mp}^{\left(\frac{3}{2}\right)} \\
& \mp 5(k-1)(k+1)(k+5) \hat{T}^{(1)}\left(\begin{array}{c}
\hat{G}_{12} \\
\hat{G}_{21}
\end{array}\right) \\
& \left.\mp \frac{4}{3}(k-1)\left(7 k^{2}+4 k+25\right) \partial \hat{T}_{\mp}^{\left(\frac{3}{2}\right)}\right](w) \\
& +\frac{6 k}{(7 k+3)}\left[ \pm \frac{i}{(k+2)} \hat{A}_{ \pm}\left(\begin{array}{c}
\hat{G}_{22} \\
\hat{G}_{11}
\end{array}\right) \mp \frac{i}{(k+2)} \hat{A}_{3}\left(\begin{array}{c}
\hat{G}_{12} \\
\hat{G}_{21}
\end{array}\right)\right. \\
& \pm \frac{i}{5} \hat{B}_{ \pm}\left(\begin{array}{c}
\hat{G}_{11} \\
\hat{G}_{22}
\end{array}\right) \mp \frac{i}{5} \hat{B}_{3}\left(\begin{array}{c}
\hat{G}_{12} \\
\hat{G}_{21}
\end{array}\right)+\partial\left(\begin{array}{c}
\hat{G}_{12} \\
\hat{G}_{21}
\end{array}\right) \\
& \left.+\frac{3(9 k+13)}{5(k+2)} \hat{T}^{(1)} \hat{T}_{\mp}^{\left(\frac{3}{2}\right)}\right](w), \\
& \left(\begin{array}{l}
\left\{\hat{G}_{11} \hat{V}_{-}^{(2)}\right\}_{-1} \\
\left\{\hat{G}_{22} \hat{U}_{+}^{(2)}\right\}_{-1}
\end{array}\right)(w)=\mp \hat{W}_{\mp}^{\left(\frac{5}{2}\right)}(w)-\frac{4(8+k)}{9(5+k)} \partial \hat{T}_{\mp}^{\left(\frac{3}{2}\right)}(w) \\
& +\frac{(k+8)}{(k+5)\left(38 k^{2}+41 k-25\right)}\left[ \pm 32 i k \hat{A}_{ \pm}\left(\begin{array}{c}
\hat{V}^{\left(\frac{3}{2}\right)} \\
\hat{U}^{\left(\frac{3}{2}\right)}
\end{array}\right)\right. \\
& \mp 32 i k \hat{A}_{3} \hat{T}_{\mp}^{\left(\frac{3}{2}\right)} \pm \frac{2}{3} i(2 k+1)(5+k) \hat{B}_{ \pm}\left(\begin{array}{c}
\hat{U}^{\left(\frac{3}{2}\right)} \\
\hat{V}^{\left(\frac{3}{2}\right)}
\end{array}\right) \\
& \pm 4 i\left(-5+5 k+6 k^{2}\right) \hat{B}_{3} \hat{T}_{\mp}^{\left(\frac{3}{2}\right)}+\frac{8}{3}(k+2)(k+5) \hat{T}^{(1)}\left(\begin{array}{c}
\hat{G}_{12} \\
\hat{G}_{21}
\end{array}\right) \\
& \left.-\frac{8}{9}\left(2 k^{2}-31 k-25\right) \partial \hat{T}_{\mp}^{\left(\frac{3}{2}\right)}\right](w), \\
& \left(\begin{array}{l}
\left\{\hat{G}_{21} \hat{V}_{-}^{(2)}\right\}_{-1} \\
\left\{\hat{G}_{12} \hat{U}_{+}^{(2)}\right\}_{-1}
\end{array}\right)(w)= \pm\left(\begin{array}{c}
\hat{V}^{\left(\frac{5}{2}\right)} \\
\hat{U}^{\left(\frac{5}{2}\right)}
\end{array}\right)(w)+\frac{4(8+k)}{9(5+k)} \partial\left(\begin{array}{c}
\hat{V}^{\left(\frac{3}{2}\right)} \\
\hat{U}^{\left(\frac{3}{2}\right)}
\end{array}\right)(w) \\
& +\frac{(k+8)}{(k+5)\left(38 k^{2}+41 k-25\right)}\left[\mp 32 i k \hat{A}_{\mp} \hat{T}_{\mp}^{\left(\frac{3}{2}\right)}\right. \\
& \mp 32 i k \hat{A}_{3}\left(\begin{array}{c}
\hat{V}^{\left(\frac{3}{2}\right)} \\
\hat{U}^{\left(\frac{3}{2}\right)}
\end{array}\right) \pm \frac{2}{3} i(k+5)(2 k+1) \hat{B}_{ \pm} \hat{T}_{ \pm}^{\left(\frac{3}{2}\right)} \\
& \mp 4 i\left(-5+5 k+6 k^{2}\right) \hat{B}_{3}\left(\begin{array}{c}
\hat{V}^{\left(\frac{3}{2}\right)} \\
\hat{U}^{\left(\frac{3}{2}\right)}
\end{array}\right)
\end{aligned}
$$




$$
\begin{aligned}
& -\frac{8}{3}(k+2)(k+5) \hat{T}^{(1)}\left(\begin{array}{c}
\hat{G}_{22} \\
\hat{G}_{11}
\end{array}\right) \\
& \left.+\frac{8}{9}\left(-25-31 k+2 k^{2}\right) \partial\left(\begin{array}{c}
\hat{V}^{\left(\frac{3}{2}\right)} \\
\hat{U}^{\left(\frac{3}{2}\right)}
\end{array}\right)\right](w) \\
& \left(\begin{array}{l}
\left\{\hat{G}_{11}\left(\hat{T}^{(2)}+\hat{W}^{(2)}\right)\right\}_{-1} \\
\left\{\hat{G}_{22}\left(\hat{T}^{(2)}+\hat{W}^{(2)}\right)\right\}_{-1}
\end{array}\right)(w)=\left(\begin{array}{c}
\hat{U}^{\left(\frac{5}{2}\right)} \\
\hat{V}^{\left(\frac{5}{2}\right)}
\end{array}\right)(w) \mp \frac{4(8+k)}{9(5+k)} \partial\left(\begin{array}{c}
\hat{U}^{\left(\frac{3}{2}\right)} \\
\hat{V}^{\left(\frac{3}{2}\right)}
\end{array}\right)(w) \\
& +\frac{(k+8)}{(k+5)\left(38 k^{2}+41 k-25\right)}\left[-32 i k \hat{A}_{ \pm} \hat{T}_{ \pm}^{\left(\frac{3}{2}\right)}\right. \\
& -32 i k \hat{A}_{3}\left(\begin{array}{c}
\hat{U}^{\left(\frac{3}{2}\right)} \\
\hat{V}^{\left(\frac{3}{2}\right)}
\end{array}\right)-4 i\left(-5+5 k+6 k^{2}\right) \hat{B}_{\mp} \hat{T}_{\mp}^{\left(\frac{3}{2}\right)} \\
& +\frac{8}{3} i\left(-5+13 k+10 k^{2}\right) \hat{B}_{3}\left(\begin{array}{c}
\hat{U}^{\left(\frac{3}{2}\right)} \\
\hat{V}^{\left(\frac{3}{2}\right)}
\end{array}\right) \\
& \pm \frac{8}{3}(2+k)(k+5) \hat{T}^{(1)}\left(\begin{array}{l}
\hat{G}_{11} \\
\hat{G}_{22}
\end{array}\right) \\
& \left.\mp \frac{8}{9}\left(2 k^{2}-31 k-25\right) \partial\left(\begin{array}{c}
\hat{U}^{\left(\frac{3}{2}\right)} \\
\hat{V}^{\left(\frac{3}{2}\right)}
\end{array}\right)\right](w) \\
& +\frac{(3+4 k)}{(7 k+3)}\left[ \pm \frac{2 i}{(k+2)} \hat{A}_{ \pm}\left(\begin{array}{c}
\hat{G}_{21} \\
\hat{G}_{12}
\end{array}\right) \pm \frac{2 i}{(k+2)} \hat{A}_{3}\left(\begin{array}{c}
\hat{G}_{11} \\
\hat{G}_{22}
\end{array}\right)\right. \\
& \mp \frac{2 i}{5} \hat{B}_{\mp}\left(\begin{array}{c}
\hat{G}_{12} \\
\hat{G}_{21}
\end{array}\right) \mp \frac{2 i}{5} \hat{B}_{3}\left(\begin{array}{c}
\hat{G}_{11} \\
\hat{G}_{22}
\end{array}\right)-2 \partial\left(\begin{array}{c}
\hat{G}_{11} \\
\hat{G}_{22}
\end{array}\right) \\
& \left.-\frac{6(9 k+13)}{5(k+2)} \hat{T}^{(1)}\left(\begin{array}{c}
\hat{U}^{\left(\frac{3}{2}\right)} \\
\hat{V}^{\left(\frac{3}{2}\right)}
\end{array}\right)\right](w) \text {, } \\
& \left(\begin{array}{l}
\left\{\hat{G}_{12}\left(\hat{T}^{(2)}+\hat{W}^{(2)}\right)\right\}_{-1} \\
\left\{\hat{G}_{21}\left(\hat{T}^{(2)}+\hat{W}^{(2)}\right)\right\}_{-1}
\end{array}\right)(w)=\hat{W}_{\mp}^{\left(\frac{5}{2}\right)}(w) \pm \frac{4(8+k)}{9(5+k)} \partial \hat{T}_{\mp}^{\left(\frac{3}{2}\right)}(w) \\
& +\frac{(k+8)}{(k+5)\left(38 k^{2}+41 k-25\right)}\left[-32 i k \hat{A}_{ \pm}\left(\begin{array}{c}
\hat{V}^{\left(\frac{3}{2}\right)} \\
\hat{U}^{\left(\frac{3}{2}\right)}
\end{array}\right)\right. \\
& +32 i k \hat{A}_{3} \hat{T}_{\mp}^{\left(\frac{3}{2}\right)}+4 i\left(-5+5 k+6 k^{2}\right) \hat{B}_{ \pm}\left(\begin{array}{c}
\hat{U}^{\left(\frac{3}{2}\right)} \\
\hat{V}^{\left(\frac{3}{2}\right)}
\end{array}\right) \\
& +\frac{8}{3} i\left(-5+13 k+10 k^{2}\right) \hat{B}_{3} \hat{T}_{\mp}^{\left(\frac{3}{2}\right)} \mp \frac{8}{3}(k+2)(k+5) \hat{T}^{(1)}\left(\begin{array}{c}
\hat{G}_{12} \\
\hat{G}_{21}
\end{array}\right) \\
& \left. \pm \frac{8}{9}\left(2 k^{2}-31 k-25\right) \partial \hat{T}_{\mp}^{\left(\frac{3}{2}\right)}\right](w) \\
& +\frac{(3+4 k)}{(7 k+3)}\left[\mp \frac{2 i}{(k+2)} \hat{A}_{ \pm}\left(\begin{array}{c}
\hat{G}_{22} \\
\hat{G}_{11}
\end{array}\right) \pm \frac{2 i}{(k+2)} \hat{A}_{3}\left(\begin{array}{c}
\hat{G}_{12} \\
\hat{G}_{21}
\end{array}\right)\right.
\end{aligned}
$$




$$
\begin{aligned}
& \mp \frac{2 i}{5} \hat{B}_{ \pm}\left(\begin{array}{c}
\hat{G}_{11} \\
\hat{G}_{22}
\end{array}\right) \pm \frac{2 i}{5} \hat{B}_{3}\left(\begin{array}{c}
\hat{G}_{12} \\
\hat{G}_{21}
\end{array}\right) \\
& \left. \pm 2 \partial\left(\begin{array}{c}
\hat{G}_{12} \\
\hat{G}_{21}
\end{array}\right)-\frac{6(9 k+13)}{5(k+2)} \hat{T}^{(1)} \hat{T}_{\mp}^{\left(\frac{3}{2}\right)}(w)\right](w) .
\end{aligned}
$$

In each OPE of appendix (C.51), the first line contains spin- $\frac{5}{2}$ current and the derivative (descendant) term from the spin- $\frac{3}{2}$ current in the second-order pole. The next lines in the OPE consist of a primary field which is a quadratic expression between the higher spin currents and the currents from large $\mathcal{N}=4$ nonlinear algebra. The other primary field which is also a quadratic in $\hat{A}_{i}(w), \hat{B}_{i}(w)$ and $\hat{G}_{a}(w)$ (as well as a derivative term and a product between the higher spin currents) appears in other OPEs.

For example, in table 4 , the fourth row describes the spin- $\frac{5}{2}$ current $\hat{W}_{+}^{\left(\frac{5}{2}\right)}(w)$ and other 12 (hatted) composite fields corresponding to the first-order pole $\left\{\hat{G}_{21}\left(\hat{T}^{(2)}-\hat{W}^{(2)}\right)\right\}_{-1}(w)$ in appendix (C.51). Among those composite fields, six of them participate in one spin$\frac{5}{2}$ primary field where the OPE between the spin- 1 and the spin- $\frac{3}{2}$ currents contains the first-order pole term $\hat{T}_{+}^{\left(\frac{3}{2}\right)}(w)$ (therefore the ordering between these currents involves the derivative term $\left.\partial \hat{T}_{+}^{\left(\frac{3}{2}\right)}(w)\right)$ and the remaining ones do in other spin- $\frac{5}{2}$ primary field containing the product of two higher spin currents $\hat{T}^{(1)} \hat{T}_{+}^{\left(\frac{3}{2}\right)}(w)$. One can easily check that the OPE $\hat{T}^{(1)}(z) \hat{T}_{+}^{\left(\frac{3}{2}\right)}(w)$ provides the first-order pole term $-\frac{1}{2} \hat{G}_{21}(w)$ and therefore the ordering between two currents can give the derivative term $\partial \hat{G}_{21}(w)$. One can see the similar features in the first-order poles of $\left\{\hat{G}_{11} \hat{V}_{+}^{(2)}\right\}_{-1}(w),\left\{\hat{G}_{22} \hat{U}_{+}^{(2)}\right\}_{-1}(w)$ or $\left\{\hat{G}_{21}\left(\hat{T}^{(2)}+\hat{W}^{(2)}\right)\right\}_{-1}(w)$. Furthermore, the table 4 describes the remaining spin- $\frac{5}{2}$ currents, $\hat{W}_{-}^{\left(\frac{5}{2}\right)}(w), \hat{U}^{\left(\frac{5}{2}\right)}(w)$ and $\hat{V}^{\left(\frac{5}{2}\right)}(w)$. Similar analysis corresponding to the remaining 12 OPEs of appendix (C.51) can be done without any difficulty.

The OPEs between the spin- $\frac{3}{2}$ currents $\hat{G}_{a}(z)$ and the above four spin- $\frac{5}{2}$ currents in appendix (C.50) or (6.8) can be obtained by

$$
\begin{aligned}
\left(\begin{array}{c}
\hat{G}_{12} \\
\hat{G}_{21}
\end{array}\right)(z)\left(\begin{array}{c}
\hat{U}^{\left(\frac{5}{2}\right)} \\
\hat{V}^{\left(\frac{5}{2}\right)}
\end{array}\right)(w)= & \pm \frac{1}{(z-w)^{2}}\left[\frac{64 k(k+1)(k+8)}{(k+5)\left(38 k^{2}+41 k-25\right)}\right]\left(\begin{array}{c}
\hat{U}_{-}^{(2)} \\
\hat{V}_{+}^{(2)}
\end{array}\right)(w) \\
& +\frac{1}{(z-w)}[\text { spin-3 composite fields }](w)+\cdots, \\
\left(\begin{array}{c}
\hat{G}_{21} \\
\hat{G}_{12}
\end{array}\right)(z)\left(\begin{array}{c}
\hat{U}^{\left(\frac{5}{2}\right)} \\
\hat{V}^{\left(\frac{5}{2}\right)}
\end{array}\right)(w)= & \mp \frac{1}{(z-w)^{2}}\left[\frac{96(k-1)(k+1)(2 k+5)}{(k+5)\left(38 k^{2}+41 k-25\right)}\right]\left(\begin{array}{c}
\hat{U}_{+}^{(2)} \\
\hat{V}_{-}^{(2)}
\end{array}\right)(w) \\
& +\frac{1}{(z-w)}[\text { spin-3 composite fields }](w)+\cdots, \\
\left(\begin{array}{c}
\hat{G}_{22} \\
\hat{G}_{11}
\end{array}\right)(z)\left(\begin{array}{c}
\hat{U}^{\left(\frac{5}{2}\right)} \\
\hat{V}^{\left(\frac{5}{2}\right)}
\end{array}\right)(w)= & \frac{1}{(z-w)^{2}} \frac{(k+1)}{(k+5)\left(38 k^{2}+41 k-25\right)} \\
& \times\left[-\frac{96(-5+3 k)\left(3+4 k+2 k^{2}\right)}{\hat{T}}\right. \\
& +48(k-1)(2 k+5)\left(\hat{T}^{(2)}+\hat{W}^{(2)}\right)
\end{aligned}
$$




$$
\begin{aligned}
& \left.+32 k(k+8)\left(\hat{T}^{(2)}-\hat{W}^{(2)}\right)\right](w) \\
& +\frac{1}{(z-w)}\left[\mp \hat{W}^{(3)}+\text { spin-3 composite fields }\right](w)+\cdots, \\
\left(\begin{array}{c}
\hat{G}_{11} \\
\hat{G}_{22}
\end{array}\right)(z) \hat{W}_{ \pm}^{\left(\frac{5}{2}\right)}(w)= & \pm \frac{1}{(z-w)^{2}}\left[\frac{96(k-1)(k+1)(2 k+5)}{(k+5)\left(38 k^{2}+41 k-25\right)}\right]\left(\begin{array}{c}
\hat{U}_{+}^{(2)} \\
\hat{V}_{-}^{(2)}
\end{array}\right)(w) \\
& +\frac{1}{(z-w)}[\operatorname{spin}-3 \text { composite fields }](w)+\cdots, \\
\left(\begin{array}{c}
\hat{G}_{12} \\
\hat{G}_{21}
\end{array}\right)(z) \hat{W}_{ \pm}^{\left(\frac{5}{2}\right)}(w)= & \frac{1}{(z-w)^{2}} \frac{(k+1)}{(k+5)\left(38 k^{2}+41 k-25\right)} \\
& \times\left[-\frac{96\left(10 k^{3}+34 k^{2}-11 k-15\right)}{\hat{\hat{T}}}\right. \\
& +48(k-1)(2 k+5)\left(\hat{T}^{(2)}+\hat{W}^{(2)}\right) \\
& +\frac{1}{(z-w)}\left[\mp \hat{W}^{(3)}+\operatorname{spin}-3 \text { composite fields }\right](w)+\cdots, \\
\left(\begin{array}{c}
\hat{G}_{22} \\
\hat{G}_{11}
\end{array}\right)(z) \hat{W}_{ \pm}^{\left(\frac{5}{2}\right)}(w)= & \mp \frac{1}{(z-w)^{2}}\left[\frac{64 k(k+1)(k+8)}{(k+5)\left(38 k^{2}+41 k-25\right)}\right]\left(\begin{array}{c}
\hat{V}_{+}^{(2)} \\
\hat{U}_{-}^{(2)}
\end{array}\right)(w) \\
& +\frac{1}{(z-w)}[\operatorname{spin}-3 \operatorname{composite} \text { fields }](w)+\cdots,
\end{aligned}
$$

where the primary field $\tilde{\hat{T}}(w)$ is given by appendix (B.35) and we do not present the complete expressions for the spin-3 composite fields consisting of 11 currents and 16 higher spin currents appearing in the first-order poles [48]. All the third-order poles appeared in previous basis are disappeared in this new basis (C.52).

Finally, the OPEs between the spin- $\frac{3}{2}$ currents $\hat{G}_{a}(z)$ and the above four spin- 3 currents in appendix (C.52) or (6.8)

$$
\begin{aligned}
\left(\begin{array}{c}
\hat{G}_{11} \\
\hat{G}_{22}
\end{array}\right)(z) \hat{W}^{(3)}(w)= & \mp \frac{1}{(z-w)^{2}}\left[\frac{(327 k+859)\left(38 k^{2}+41 k-25\right)}{4\left(786 k^{3}+3727 k^{2}+2920 k-1925\right)}\right]\left(\begin{array}{c}
\hat{U}^{\left(\frac{5}{2}\right)} \\
\hat{V}^{\left(\frac{5}{2}\right)}
\end{array}\right)(w) \\
& +\frac{1}{(z-w)}\left[\text { spin- } \frac{7}{2} \text { composite fields }\right](w)+\cdots, \\
\left(\begin{array}{c}
\hat{G}_{12} \\
\hat{G}_{21}
\end{array}\right)(z) \hat{W}^{(3)}(w)= & \pm \frac{1}{(z-w)^{2}}\left[\frac{(327 k+859)\left(38 k^{2}+41 k-25\right)}{4\left(786 k^{3}+3727 k^{2}+2920 k-1925\right)}\right] \hat{W}_{\mp}^{\left(\frac{5}{2}\right)}(w) \\
& +\frac{1}{(z-w)}\left[\operatorname{spin}-\frac{7}{2} \text { composite fields }\right](w)+\cdots .
\end{aligned}
$$

Note that the structure constants appearing in the second-order poles have same values in appendix (C.53) and we do not present the complete expressions for the spin- $\frac{7}{2}$ composite fields [48]. All the third-order poles appeared in previous basis are disappeared in this new basis (C.53). 
Open Access. This article is distributed under the terms of the Creative Commons Attribution License (CC-BY 4.0), which permits any use, distribution and reproduction in any medium, provided the original author(s) and source are credited.

\section{References}

[1] M.R. Gaberdiel and R. Gopakumar, Large-N=4 Holography, JHEP 09 (2013) 036 [arXiv: 1305.4181] [INSPIRE].

[2] M.R. Gaberdiel and R. Gopakumar, An AdS 3 Dual for Minimal Model CFTs, Phys. Rev. D 83 (2011) 066007 [arXiv: 1011.2986] [InSPIRE].

[3] M.R. Gaberdiel and R. Gopakumar, Triality in Minimal Model Holography, JHEP 07 (2012) 127 [arXiv:1205.2472] [INSPIRE].

[4] M.R. Gaberdiel and R. Gopakumar, Minimal Model Holography, J. Phys. A 46 (2013) 214002 [arXiv:1207.6697] [InSPIRE].

[5] M. Ammon, M. Gutperle, P. Kraus and E. Perlmutter, Black holes in three dimensional higher spin gravity: A review, J. Phys. A 46 (2013) 214001 [arXiv:1208.5182] [INSPIRE].

[6] A. Sevrin, W. Troost and A. Van Proeyen, Superconformal Algebras in Two-Dimensions with $N=4$, Phys. Lett. B 208 (1988) 447 [inSPIRE].

[7] P. Goddard and A. Schwimmer, Factoring Out Free Fermions and Superconformal Algebras, Phys. Lett. B 214 (1988) 209 [inSPIRE].

[8] M. Ademollo et al., Supersymmetric Strings and Color Confinement, Phys. Lett. B 62 (1976) 105 [INSPIRE].

[9] M. Ademollo et al., Dual String with U(1) Color Symmetry, Nucl. Phys. B 111 (1976) 77 [INSPIRE].

[10] K. Schoutens, $O(n)$ Extended Superconformal Field Theory in Superspace, Nucl. Phys. B 295 (1988) 634 [inSPIRE].

[11] E.A. Ivanov and S.O. Krivonos, $N=4$ Superliouville Equation (in Russian), J. Phys. A 17 (1984) L671 [inSPIRE].

[12] E.A. Ivanov and S.O. Krivonos, $N=4$ Superextension of the Liouville Equation With Quaternionic Structure, Theor. Math. Phys. 63 (1985) 477 [InSPIRE].

[13] E.A. Ivanov, S.O. Krivonos and V.M. Leviant, A New Class of Superconformal $\sigma$ Models With the Wess-Zumino Action, Nucl. Phys. B 304 (1988) 601 [InSPIRE].

[14] E.A. Ivanov, S.O. Krivonos and V.M. Leviant, Quantum $N=3, N=4$ Superconformal WZW $\sigma$-models, Phys. Lett. B 215 (1988) 689 [Erratum ibid. B 221 (1989) 432] [INSPIRE].

[15] A. Sevrin and G. Theodoridis, N=4 Superconformal coset theories, Nucl. Phys. B 332 (1990) 380 [inSPIRE].

[16] Y. Kazama and H. Suzuki, New $N=2$ Superconformal Field Theories and Superstring Compactification, Nucl. Phys. B 321 (1989) 232 [inSPIRE].

[17] Y. Kazama and H. Suzuki, Characterization of $N=2$ Superconformal Models Generated by Coset Space Method, Phys. Lett. B 216 (1989) 112 [InSPIRE].

[18] J.A. Wolf, Complex Homogeneous Contact Manifolds and Quaternionic Symmetric Spaces, J. Math. Mech. 14 (1965) 1033. 
[19] D.V. Alekseevskii, Classification of Quarternionic Spaces with a Transitive Solvable Group of Motions, Math. USSR Izv. 9 (1975) 297.

[20] S. Salamon, Quaternionic Kahler Manifolds, Invent. Math. 67 (1982) 143.

[21] J. Bagger and E. Witten, Matter Couplings in $N=2$ Supergravity, Nucl. Phys. B 222 (1983) 1 [InSPIRE].

[22] M. Roček, C.-h. Ahn, K. Schoutens and A. Sevrin, Superspace WZW models and black holes, hep-th/9110035 [INSPIRE].

[23] C. Ahn, Extended conformal symmetry in two-dimensional quantum field theory, Ph.D. Thesis, State University of New York at Stony Brook (1992), UMI-93-10057 [INSPIRE].

[24] S.J. Gates Jr. and S.V. Ketov, No $N=4$ strings on wolf spaces, Phys. Rev. D 52 (1995) 2278 [hep-th/9501140] [INSPIRE].

[25] W. Lerche, C. Vafa and N.P. Warner, Chiral Rings in $N=2$ Superconformal Theories, Nucl. Phys. B 324 (1989) 427 [INSPIRE].

[26] R.E.C. Perret, A Classical $N=4$ superW algebra, Int. J. Mod. Phys. A 8 (1993) 3615 [hep-th/9211128] [INSPIRE].

[27] R.E.C. Perret, Dual formulation of classical W algebras, Lett. Math. Phys. 27 (1993) 27 [hep-th/9208068] [INSPIRE].

[28] M. Naka, Remarks on $N=1 S W$ (3/2,2) algebras, JHEP 12 (2002) 059 [hep-th/0204202] [INSPIRE].

[29] M. Henneaux, G. Lucena Gómez, J. Park and S.-J. Rey, Super- W(infinity) Asymptotic Symmetry of Higher-Spin AdS $S_{3}$ Supergravity, JHEP 06 (2012) 037 [arXiv:1203.5152] [INSPIRE].

[30] K. Thielemans, A Mathematica package for computing operator product expansions, Int. J. Mod. Phys. C 2 (1991) 787 [inSPIRE].

[31] P. Spindel, A. Sevrin, W. Troost and A. Van Proeyen, Extended Supersymmetric $\sigma$-models on Group Manifolds. 1. The Complex Structures, Nucl. Phys. B 308 (1988) 662 [InSPIRE].

[32] L. Anguelova, M. Roček and S. Vandoren, HyperKähler cones and orthogonal Wolf spaces, JHEP 05 (2002) 064 [hep-th/0202149] [INSPIRE].

[33] L. Jarv, T. Mohaupt and F. Saueressig, Effective supergravity actions for flop transitions, JHEP 12 (2003) 047 [hep-th/0310173] [INSPIRE].

[34] C.M. Hull and B.J. Spence, $N=2$ Current Algebra and Coset Models, Phys. Lett. B 241 (1990) 357 [INSPIRE].

[35] C. Ahn, The Large-N 't Hooft Limit of Kazama-Suzuki Model, JHEP 08 (2012) 047 [arXiv:1206.0054] [INSPIRE].

[36] C. Ahn, The Operator Product Expansion of the Lowest Higher Spin Current at Finite N, JHEP 01 (2013) 041 [arXiv:1208.0058] [INSPIRE].

[37] C.-h. Ahn, Explicit construction of $N=2 W(3)$ current in the $N=2$ coset $\mathrm{SU}(3) / \mathrm{SU}(2) \times U(1)$ model, Phys. Lett. B 348 (1995) 77 [hep-th/9410170] [INSPIRE].

[38] F. Bastianelli and N. Ohta, The Large- $N=4$ superconformal algebra and its BRST operator, Phys. Rev. D 50 (1994) 4051 [hep-th/9402118] [InSPIRE]. 
[39] A. Sevrin, W. Troost, A. Van Proeyen and P. Spindel, Extended supersymmetric $\sigma$-models on group manifolds. 2. Current algebras, Nucl. Phys. B 311 (1988) 465 [INSPIRE].

[40] A. Ali and A. Kumar, A New $N=4$ superconformal algebra, Mod. Phys. Lett. A 8 (1993) 1527 [hep-th/9301010] [INSPIRE].

[41] A. Van Proeyen, Realizations of $N=4$ Superconformal Algebras on Wolf Spaces, Class. Quant. Grav. 6 (1989) 1501 [inSPIRE].

[42] M. Günaydin, J.L. Petersen, A. Taormina and A. Van Proeyen, On the Unitary Representations of a Class of $N=4$ Superconformal Algebras, Nucl. Phys. B 322 (1989) 402 [INSPIRE].

[43] V.G. Knizhnik, Superconformal Algebras in Two-dimensions, Theor. Math. Phys. 66 (1986) 68 [InSPIRE].

[44] M.A. Bershadsky, Superconformal Algebras in Two-dimensions With Arbitrary N, Phys. Lett. B 174 (1986) 285 [inSPIRE].

[45] K. Schoutens, A Nonlinear Representation of the $d=2 \mathrm{SO}(4)$ Extended Superconformal Algebra, Phys. Lett. B 194 (1987) 75 [inSPIRE].

[46] K. Schoutens, Extensions of conformal symmetry in two dimensional quantum field theory, Ph.D. Thesis, University of Utrecht, Netherlands, May 1989.

[47] L.J. Romans, The $N=2$ superW(3) algebra, Nucl. Phys. B 369 (1992) 403 [InSPIRE].

[48] C. Ahn, Higher Spin Currents in Wolf Space. Part II, in preparation.

[49] P. Bowcock, Quasi-primary Fields and Associativity of Chiral Algebras, Nucl. Phys. B 356 (1991) 367 [InSPIRE].

[50] R. Blumenhagen, M. Flohr, A. Kliem, W. Nahm, A. Recknagel and R. Varnhagen, $W$ algebras with two and three generators, Nucl. Phys. B 361 (1991) 255 [INSPIRE].

[51] P. Bouwknegt and K. Schoutens, W symmetry in conformal field theory, Phys. Rept. 223 (1993) 183 [hep-th/9210010] [INSPIRE].

[52] W. Nahm, Algebras of two-dimensional chiral fields and their classification, in proceedings of $3^{\text {rd }}$ Regional Conference on Mathematical physics, Islamabad, Pakistan, 17-24 February 1989 [INSPIRE].

[53] W. Nahm, Chiral algebras of two-dimensional chiral field theories and their normal ordered products, in proceedings of Recent developments in conformal field theories, Trieste, Italy (1989), p. 81-84 [INSPIRE].

[54] C. Ahn, The Higher Spin Currents in the $N=1$ Stringy Coset Minimal Model, JHEP 04 (2013) 033 [arXiv:1211.2589] [INSPIRE].

[55] C. Ahn and J. Paeng, Higher Spin Currents in the Holographic $\mathcal{N}=1$ Coset Minimal Model, JHEP 01 (2014) 007 [arXiv: 1310.6185] [INSPIRE].

[56] H.J. Boonstra, B. Peeters and K. Skenderis, Brane intersections, anti-de Sitter space-times and dual superconformal theories, Nucl. Phys. B 533 (1998) 127 [hep-th/9803231] [INSPIRE].

[57] S. Elitzur, O. Feinerman, A. Giveon and D. Tsabar, String theory on $A d S_{3} \times S^{3} \times S^{3} \times S^{1}$, Phys. Lett. B 449 (1999) 180 [hep-th/9811245] [INSPIRE]. 
[58] J. de Boer, A. Pasquinucci and K. Skenderis, AdS/CFT dualities involving large $2-D$ $N=4$ superconformal symmetry, Adv. Theor. Math. Phys. 3 (1999) 577 [hep-th/9904073] [INSPIRE].

[59] S. Gukov, E. Martinec, G.W. Moore and A. Strominger, The Search for a holographic dual to $A d S_{3} \times S^{3} \times S^{3} \times S^{1}$, Adv. Theor. Math. Phys. 9 (2005) 435 [hep-th/0403090] [InSPIRE].

[60] M.R. Gaberdiel, Large N=4 Minimal Model Holography, talk given at the conference Integrability in Gauge and String Theory 2013, Utrecht, Netherlands, 19-23 August 2013 [http://www.staff.science.uu.nl/ SSfond101/igst13/gaberdiel.pdf].

[61] A. Babichenko, B. Stefański Jr. and K. Zarembo, Integrability and the $A d S_{3} / C F T_{2}$ correspondence, JHEP 03 (2010) 058 [arXiv:0912.1723] [INSPIRE].

[62] O. Ohlsson Sax and B. Stefański Jr., Integrability, spin-chains and the AdS3/CFT2 correspondence, JHEP 08 (2011) 029 [arXiv: 1106.2558] [INSPIRE].

[63] F.A. Bais, P. Bouwknegt, M. Surridge and K. Schoutens, Extensions of the Virasoro Algebra Constructed from Kac-Moody Algebras Using Higher Order Casimir Invariants, Nucl. Phys. B 304 (1988) 348 [INSPIRE].

[64] F.A. Bais, P. Bouwknegt, M. Surridge and K. Schoutens, Coset Construction for Extended Virasoro Algebras, Nucl. Phys. B 304 (1988) 371 [InSPIRE].

[65] C. Ahn, The Coset Spin-4 Casimir Operator and Its Three-Point Functions with Scalars, JHEP 02 (2012) 027 [arXiv:1111.0091] [INSPIRE].

[66] C. Ahn, Higher Spin Currents with Arbitrary $N$ in the $\mathcal{N}=\infty$ Stringy Coset Minimal Model, JHEP 07 (2013) 141 [arXiv:1305.5892] [INSPIRE].

[67] C. Ahn and H. Kim, Spin-5 Casimir operator its three-point functions with two scalars, JHEP 01 (2014) 012 [arXiv: 1308.1726] [INSPIRE].

[68] C. Ahn, The Large-N 't Hooft Limit of Coset Minimal Models, JHEP 10 (2011) 125 [arXiv: 1106.0351] [INSPIRE].

[69] C. Ahn, The Primary Spin-4 Casimir Operators in the Holographic $\mathrm{SO}(N)$ Coset Minimal Models, JHEP 05 (2012) 040 [arXiv: 1202.0074] [INSPIRE].

[70] C. Ahn and J. Paeng, The OPEs of Spin-4 Casimir Currents in the Holographic $\mathrm{SO}(N)$ Coset Minimal Models, Class. Quant. Grav. 30 (2013) 175004 [arXiv:1301.0208] [INSPIRE].

[71] C.-h. Ahn, E. Ivanov and A.S. Sorin, $N=2$ affine superalgebras and Hamiltonian reduction in $N=2$ superspace, Commun. Math. Phys. 183 (1997) 205 [hep-th/9508005] [InSPIRE].

[72] F. Defever, W. Troost and Z. Hasiewicz, Superconformal algebras with quadratic nonlinearity, Phys. Lett. B 273 (1991) 51 [InSPIRE].

[73] M. Günaydin and S.V. Ketov, Seven sphere and the exceptional $N=7$ and $N=8$ superconformal algebras, Nucl. Phys. B 467 (1996) 215 [hep-th/9601072] [INSPIRE].

[74] B. Bina and M. Günaydin, Real forms of nonlinear superconformal and quasisuperconformal algebras and their unified realization, Nucl. Phys. B 502 (1997) 713 [hep-th/9703188] [INSPIRE].

[75] E.S. Fradkin and V.Y. Linetsky, An Exceptional $N=8$ superconformal algebra in two-dimensions associated with F(4), Phys. Lett. B 275 (1992) 345 [INSPIRE].

[76] P. Bowcock, Exceptional superconformal algebras, Nucl. Phys. B 381 (1992) 415 [hep-th/9202061] [INSPIRE]. 